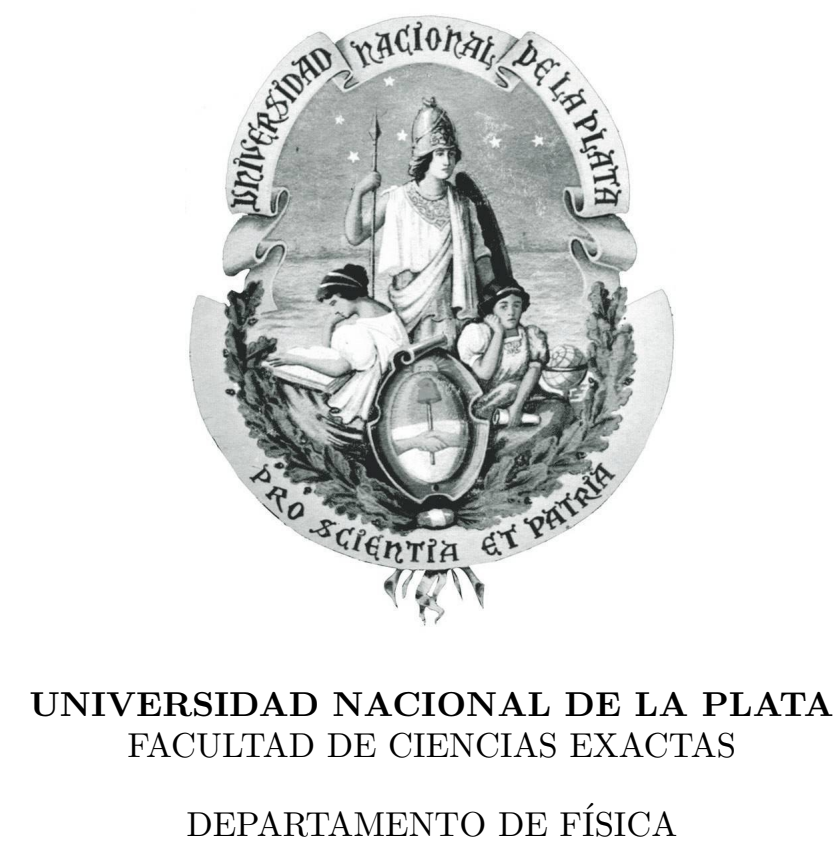

TRABAJO DE TESIS DOCTORAL:

\title{
Aplicaciones de la Correspondencia AdS/CFT en el ámbito de la Física de la Materia Condensada
}

Tesis de doctorado presentada por Gastón Luciano Giordano para optar por el título de Doctor en Física por la Universidad Nacional de La Plata

Director: Dr. Adrián René Lugo

Año: 2018 

La presente Tesis se basa en los siguientes artículos:

G. L. Giordano, A. R. Lugo: "Holographic phase transitions from higgsed, non abelian charged black holes": JHEP 1507, 172 (2015) doi:10.1007/JHEP07(2015)172 [arXiv:1501.04033 [hep-th]].

G. L. Giordano, N. E. Grandi, A. R. Lugo: "Fermionic spectral functions in backreacting p-wave superconductors at finite temperature", JHEP 1704, 087 (2017) doi:10.1007/JHEP04(2017)087 [arXiv:1610.04268 [hep-th]].

G. L. Giordano, N. E. Grandi and A. R. Lugo, R. Soto-Garrido: "Spectral functions in doped holographic metals, and the strange metal crossover", en preparación

G. L. Giordano, A. R. Lugo, N. E. Grandi, Pablo Rodríguez-Ponte, Ignacio Salazar-Landea: "Holographic fermionic instabilities", en preparación. 


\section{Índice}

1 Introducción $\quad 6$

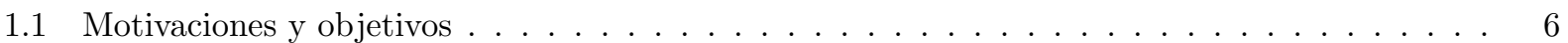

2 Teoría de Cuerdas y AdS/CFT $\quad 8$

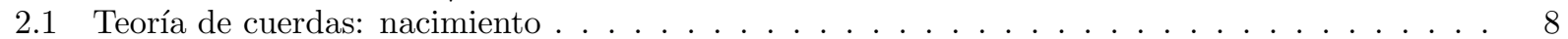

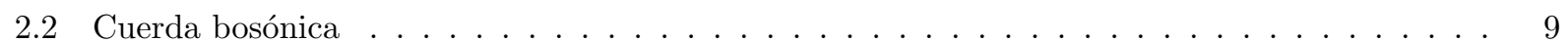

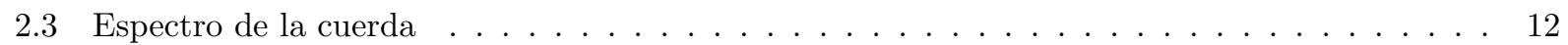

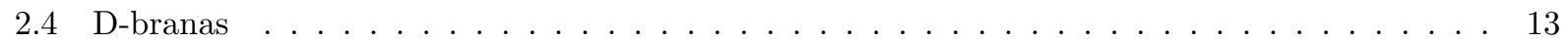

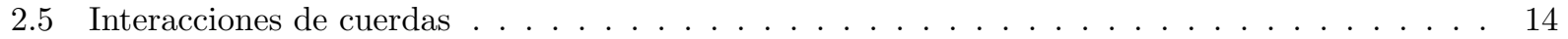

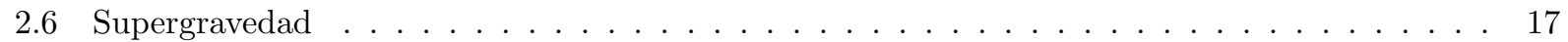

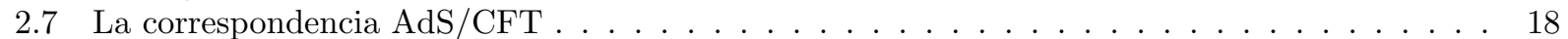

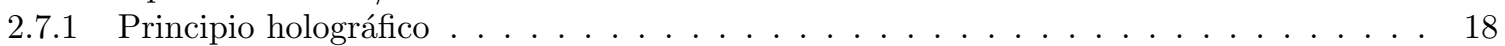

2.7.2 Temperatura de Hawking y formalismo euclídeo . . . . . . . . . . . . . . . . . . . . 18

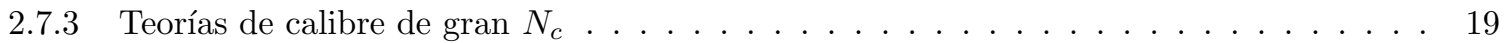

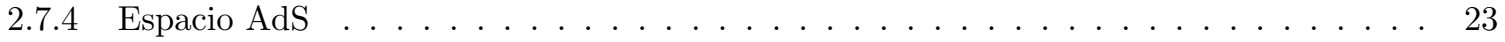

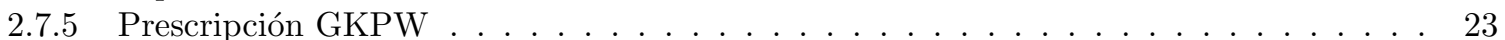

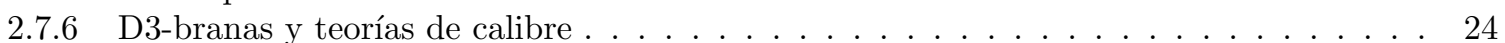

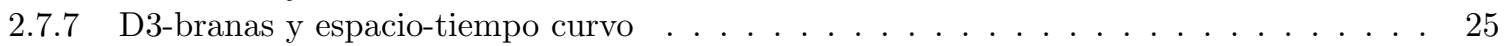

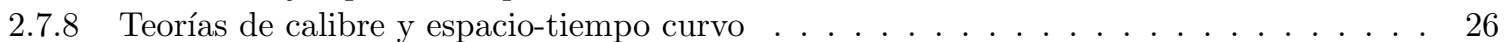

2.7.9 Cálculo de funciones de correlación usando $A d S / C F T \ldots \ldots \ldots \ldots \ldots$

3 Materia Condensada $\quad 29$

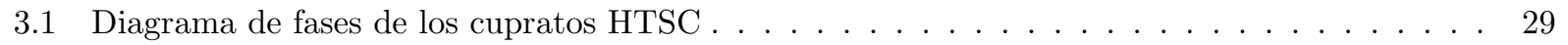

3.2 Transiciones de fase: modelo de Ginzburg-Landau . . . . . . . . . . . . . . . . . . . 29

3.3 Modelo de Ginzburg-Landau para la Superconductividad . . . . . . . . . . . . . . . . . . . 30

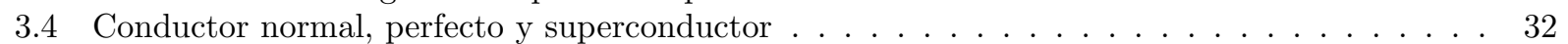

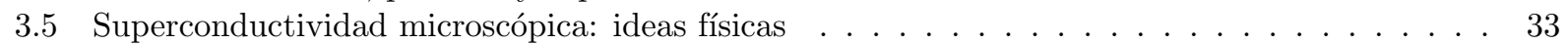

3.6 Gas de Fermi . . . . . . . . . . . . . . . . . . . . . . . . . . 34

3.7 Líquido de Fermi y metales extraños . . . . . . . . . . . . . . . . . . . . . . 34

4 Modelos holográficos de la Materia Condensada $\quad 37$

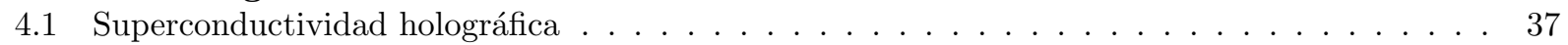

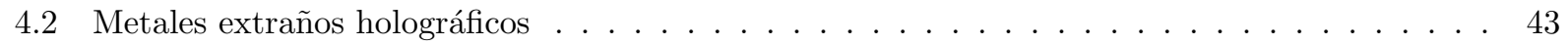

4.3 Estrella de electrones . . . . . . . . . . . . . . . . . . . . . . . . 46

5 Transiciones holográficas en presencia de campo un campo de Higgs en un agujero negro cargado no abeliano $\mathbf{5 0}$

5.1 Introducción . . . . . . . . . . . . . . . . . . . . . . . . . . . . 50

5.2 Superconductores holográficos: gravedad+Yang-Mills+Higgs . . . . . . . . . . . . . . . 51

$5.2 .1 \quad$ El modelo . . . . . . . . . . . . . . . . . . . . . . . . . . . . . . 51

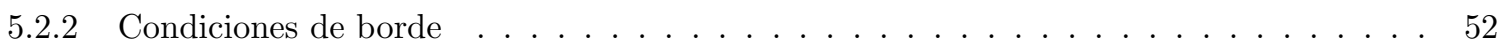

5.3 Soluciones a $T>0$ : estado superconductor $\ldots \ldots \ldots \ldots \ldots \ldots \ldots \ldots$

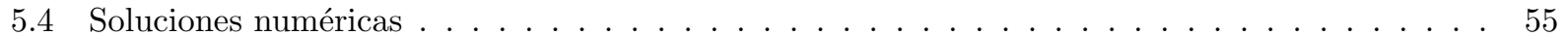

5.4 .1 La energía libre . . . . . . . . . . . . . . . . . . . . . . . . . . . . 59

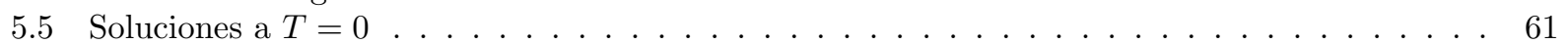

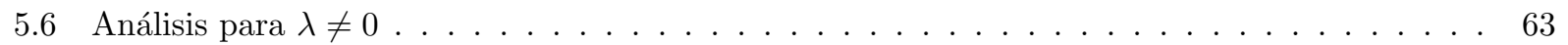

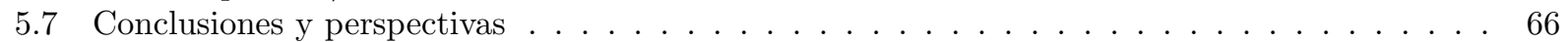

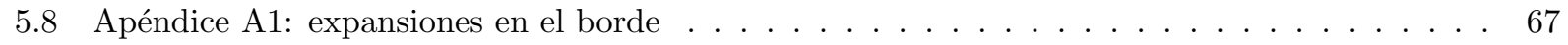

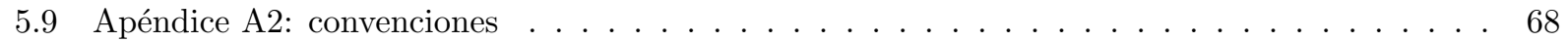

6 Funciones espectrales de superconductores holográficos de onda- $p \quad \mathbf{7 0}$

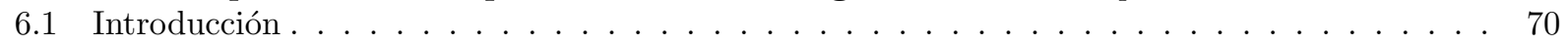

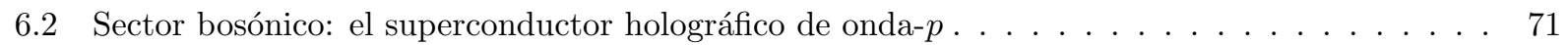

6.3 Solución gravitacional del espacio-tiempo . . . . . . . . . . . . . . . . . . . 71

6.3 .1 Entropía de Beckenstein-Hawking nula . . . . . . . . . . . . . . . . . 72

6.3.2 Entropía de Beckenstein-Hawking no nula . . . . . . . . . . . . . . . . . . . . 74

6.4 La interpretación del borde en términos de una QFT . . . . . . . . . . . . . . . . 74

6.5 Sector fermiónico: añandiendo fermiones al superconductor holográfico de onda $p \ldots \ldots$ 
6.6 Espinores de Dirac en un fondo gravitacional . . . . . . . . . . . . . . . . . . 76

6.6.1 Condiciones de borde entrantes para entropía de Beckenstein-Hawking nula . . . . . . 77

6.6.2 Condiciones de borde entrantes para entropía de Beckenstein-Hawking no nula . . . . 79

6.6.3 El comportamiento ultravioleta . . . . . . . . . . . . . . . . . . 79

6.6.4 Modos normales y los conos de Dirac . . . . . . . . . . . . . . . . . . . . . 79

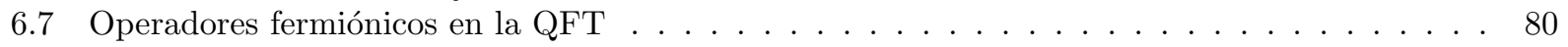

6.8 Resultados numéricos . . . . . . . . . . . . . . . . . . . . . 81

6.9 Discusión . . . . . . . . . . . . . . . . . . . . . . . 95

6.10 Apéndice B1: Acerca de la positividad de la función espectral . . . . . . . . . . . . . . . 95

6.11 Apéndice B2: Sobre la función espectral en un espacio-tiempo conforme . . . . . . . . . . . 96

7 Funciones espectrales en metales holográficos dopados y el cruce en la fase de metal extraño

7.1 Introducción . . . . . . . . . . . . . . . . . . . . . . . . . . . . . . 98

7.2 Metales no balanceados . . . . . . . . . . . . . . . . . . . . . . 98

7.3 Viendo el cruce a $T=0$ : resultados numéricos . . . . . . . . . . . . . . . . . . . 101

7.4 Viendo el crossover a $T>0$ : resultados numéricos . . . . . . . . . . . . . . . . . 102

7.5 Conclusiones . . . . . . . . . . . . . . . . . . . . . . . . . 102

7.6 Apéndice C1: Convenciones . . . . . . . . . . . . . . . . . . . . . . . . . . . 102

7.7 Apéndice C2: Una ecuación de Schrödinger para fermiones . . . . . . . . . . . . . . . . . 104

7.8 Apéndice C3: Funciones espectrales a baja energía . . . . . . . . . . . . . . . 105

8 Inestabilidades de Pomeranchuk holográficas $\quad 110$

8.1 Introducción . . . . . . . . . . . . . . . . . . . . . . . . 110

8.2 Marco general . . . . . . . . . . . . . . . . . . . . . . . . . . 110

8.3 Espinores acoplados en un espacio curvo . . . . . . . . . . . . . . . . . . . . . . . 111

8.4 Estados fermiónicos libres en la aproximación WKB . . . . . . . . . . . . . . . . . 111

8.5 Interacciones de fermiones en el espacio-tiempo holográfico. . . . . . . . . . . . . . . . . . . . 112

8.6 Apéndice D1: el método de Pomeranchuk . . . . . . . . . . . . . . . . . . . . . . . . . . . 114

8.7 Apéndice D2: estados fermiónicos libres en el espacio-tiempo curvo holográfico . . . . . . . 115

8.8 Apéndice D3: la ecuación de Dirac . . . . . . . . . . . . . . . . . . . . . . . . . 116

8.9 Apéndice D4: condiciones de borde . . . . . . . . . . . . . . . . . . . . . . . 118

8.10 Apéndice D5: ecuación de Schrödinger efectiva . . . . . . . . . . . . . . . . . . . . 119

8.11 Apéndice D6: forma general de la solución . . . . . . . . . . . . . . . . . . . 119

8.12 Apéndice D7: solución WKB . . . . . . . . . . . . . . . . . . . . . . . . 120

8.13 Apéndice D8: interacciones de fermiones en el espacio de fondo holográfico . . . . . . . . . . 124

8.14 Apéndice D9: aproximación de campo medio . . . . . . . . . . . . . . . . . . . . . 125

8.15 Apéndice D10: construcción de Pomeranchuk . . . . . . . . . . . . . . . . . . . . 128 


\section{Resumen}

En esta tesis se consideraron varios tópicos asociados a la aplicación de la correspondencia $A d S / C F T$ a sistemas de materia condensada. En particular se estudiaron modelos holográficos de superconductores, metales extraños e inestabilidades fermiónicas que pueden presentar los mismos.

El capítulo 1 es una introducción donde se exponen los objetivos y motivaciones para estudiar estos sistemas.

En el capítulo 2 se resumen las ideas y conceptos fundamentales para entender la teoría de cuerdas y la correspondencia $A d S / C F T$.

En el capítulo 3 se introducen conceptos básicos de la teoría de la Materia Condensada pertinentes a la Tesis. En primer lugar los superconductores, cuáles son los fenómenos físicos detrás de ellos y cómo es el marco teórico-matemático para describirlos. Posteriormente se introducen los líquidos de Fermi, su descripción a través de la teoría de Landau, y la existencia de una fase denominada de metal extraño que experimentan los superconductores de alta temperatura, donde dicha teoría no es aplicable.

En el capítulo 4 se introducen los modelos holográficos más básicos para describir superconductores, metales extraños y sistemas de electrones a potencial químico finito.

En el capítulo 5 se presenta el análisis de un sistema del tipo gravedad-Yang-Mills-Higgs que modela holográficamente un superconductor de onda $p / p+i p$. En tal sistema se calculan varias propiedades físicas de interés y se hace una comparación entre ellas. Se estudian además soluciones a temperatura y entropía nulas.

En el capítulo 6 se analizan funciones espectrales de campos fermiónicos a temperatura finita en el fondo de un superconductor de onda $\mathrm{p}$, teniendo en cuenta el efecto sobre la geometría de los campos de calibre.

En el capítulo 7 se estudia el cruce entre un líquido de Fermi y un metal extraño introduciendo una variable de dopaje desde el lado gravitatorio.

Por último, en el capítulo 8 se generaliza el método de Pomeranchuk en un espacio-tiempo curvo específico, para poder estudiar inestabilidades de la superficie de Fermi de un sistema de electrones fuertemente acoplado que pueden dar lugar a fases nemáticas o esmécticas. 


\section{Introducción}

\section{$1.1 \quad$ Motivaciones y objetivos}

El estudio de sistemas de electrones fuertemente correlacionados (SCES) es uno de los más fascinantes tópicos de la Física Moderna. Un ejemplo de SCES son los superconductores de alta temperatura (HTSC). Es conocido que la teoría BCS (Bardeen-Cooper-Schrieffer) de los superconductores de baja temperatura no se ajusta bien a la fenomenología observada en los HTSC, y que la descripción correcta debe tener en cuenta la alta correlación que existe entre los electrones en el estado fundamental, que resulta en un hamiltoniano fuertemente interactuante para las excitaciones. En estos SCES el tratamiento perturbativo usual en teoría cuántica de campos (QFT) no se aplica, y la investigación se ha diversificado en varias direcciones de aproximaciones alternativas [1].

Estos materiales genéricamente presentan una rica estructura en su diagrama de fases (FD). Se piensa que la dinámica de fermiones fuertemente acoplados es responsable de esta rica estructura. Aunque ha habido importantes progresos en los últimos 30 años (ver por ejemplo [2] y referencias) todavía es necesario un completo entendimiento de los HTSC debido a las dificultades que presenta su complejo FD. Por ejemplo, en el caso de los cupratos el diagrama incluye varias regiones, tales como una fase de antiferromagneto (AF), una región de pseudogap, la fase de superconductor de onda-d, la de metal extraño y la fase de líquido de Fermi (ver figura (14) y figura 2 en referencia [2]). Por otro lado, la "fase normal" (no ordenada) es también compleja. Aunque para altos dopajes es un líquido de Fermi descripto por la teoría de Landau, para dopajes intermedios una fase de metal extraño está presente, donde dicha teoría no funciona (por ejemplo, la resistividad escalea línealmente con la temperatura, mientras que en la teoría de Landau se predice un comportamiento cuadrático; debido a esto, son algunas veces llamados "non-Fermi liquids"). La fase de metal extraño ha sido observada en diferentes experimentos, especialmente en los experimentos de angle-resolved photoemission spectroscopy (ARPES).

Por otro lado existen regiónes del diagrama de fases en las cuales la simetría rotacional está rota, las llamadas fases "nemáticas", así como también fases inhomogéneas llamadas "esmécticas", terminología prestada de la teoría de cristales líquidos. Ha sido propuesto que la transición desde una fase normal de líquido de Fermi a una fase nemática es debido a la presencia de una inestabilidad de Pomeranchuk [3]. Tales inestabilidades surgen cuando un líquido de Fermi es perturbado con una excitación fermiónica representada por una deformación anisotrópica de su superficie de Fermi, la cual induce un decrecimiento neto de la energía del sistema.

En este contexto resulta de particular importancia la descripción de los grados de libertad fermiónicos tanto en la fase normal como en la fase superconductora, el análisis de la superficie de Fermi y de su deformación a temperatura finita, así como en la fase de metal extraño, y a su vez el análisis de las inestabilidades de la superficie de Fermi que se puedan presentar. La presente tesis se aboca al estudio de estos problemas.

La correspondencia AdS/CFT [4][5][6] es una poderosa herramienta para estudiar sistemas fuertemente interactuantes. Rudamente hablando, la misma establece que QFT que vive en $d$ dimensiones en un régimen de acoplamiento fuerte es equivalente a una teoría de gravedad débilmente acoplada en al menos una dimensión más alta. La solución de fondo de la teoría gravedad tiende asintóticamente a un espacio-tiempo de Anti-de Sitter $(A d S)$, mientras que la teoría de campos conforme $(C F T)$ asociada vive en la frontera conformemente minkoswkiana de $A d S$. Como veremos en el Capítulo 2, fue inicialmente conjeturada estudiando sistemas de cuerdas y branas supersimetrícas, y posteriormente extendida a dualidades gravedad/QFT en general. Cobró nuevo impulso en los últimos tiempos por las aplicaciones a la teoría de materia condensada (CMT), por lo que se la refiere frecuentemente en este contexto como dualidad AdS/CMT.

Los superconductores holográficos [7]-[10] son una clase de modelos teóricos que comparten muchas características similares con los HTSC. En particular, son sistemas fuertemente acoplados en los cuales una fase superconductora surge a bajas temperaturas. Además, los estados normales y superconductores pueden propagar fermiones que no son descriptos por la teoría de Landau. La principal diferencia con los sistemas de laboratorio es que, debido a que los superconductores holográficos son descriptos por medio de la correspondencia $A d S / C F T$, ellos requieren un gran número de grados de libertad en cualquier punto del espacio, condición referida como el "large $N$ limit" (límite de gran $N$ ).

El modelo más simple de superconductor holográfico es provisto por una teoría de Einstein-Maxwell acoplada a un campo escalar cargado, que en el marco de la correspondencia AdS/CFT es dual a un operador escalar cargado bajo una simetría global $U(1)$ (dual de la simetría de calibre) y cuyo valor de expectación de vacío es identificado con el parámetro de orden de la transición superconductora. Ha sido probado que una solución de agujero negro cargado, interpretada como una fase no condensada, se vuelve inestable y desarrolla "pelo" escalar a bajas temperaturas rompiendo la simetría $U(1)$ cerca del horizonte del agujero negro [11] [12]. Este fenómeno en general puede ser interpretado como una transición de fase de 
segundo orden entre las fases conductora y superconductora. Esta interpretación es avalada por el análisis del comportamiento de la conductividad y por la respuesta del sistema frente a un campo magnético externo [8].

Pasando al contenido de la tesis, los capítulos 2, 3 y 4 contienen material introductorio relevante para el desarrollo de la misma, donde se exponen tópicos de teoría de cuerdas, materia condensada y modelos holográficos.

En el trabajo desarrollado en el capítulo 5 estuvimos interesados en los superconductores holográficos de onda $p$, donde el condensado es alguna componente de un campo vectorial. Para que éste se encuentre cargado, un campo de calibre no abeliano es requerido, sistema al que acoplamos un campo de Higgs en la representación adjunta. El objetivo fue construir el diagrama de fases del sistema, comparando las energía libres de fases isotrópicas (superconductor de onda $\mathrm{p}+\mathrm{ip}$ ) y anisotrópicas (superconductor de onda $\mathrm{p}$ ) en todo el espacio de parámetros (masas, acoplamiento de Higgs, etc) para diferentes temperaturas.

El objetivo del trabajo desarrollado en el capítulo 6 fue considerar la inclusión de fermiones acoplados a la teoría considerada en el capítulo 4 (sin la presencia del campo de Higgs). Dichos campos fermiónicos son duales a operadores fermiónicos de la teoría de campos. El estudio de fermiones en un fondo gravitatorio de agujero negro permite, calculando la función de Green de dos puntos y utilizando la dualidad gravedad/QFT, calcular funciones de correlación fermiónicas a temperatura finita en la QFT y obtener así las funciones espectrales. Las mismas permiten estudiar holográficamente el tipo de sistema fermiónico que se está describiendo, indicando la existencia o no de superficies de Fermi y de cuasi-partículas, es decir, establecer si el sistema se comporta o no como un líquido de Fermi, etc.. Funciones espectrales en la fase superconductora de onda-s a temperatura nula fueron estudiadas en [13], mientras el caso de superconductores de onda $p$ fue considerado en [14].

Posteriormente el foco fue puesto en sistemas no balanceados, como se elabora en el capítulo 7 , los cuales se definen como sistemas de materia condensada descriptos por una teoría gravitacional dual que contiene dos campos de calibre abelianos, uno dual a la corriente de electrones y otro dual a la corriente de impurezas. El objetivo fue analizar si mediante la introducción de una variable de dopaje desde el lado gravitatorio es posible describir un cruce ("crossover") de una región donde el sistema se comporta como un líquido de Fermi a otra región donde se comporta como un metal extraño conforme variamos el dopaje, tanto a temperatura nula como a temperatura no nula.

Por último se estudiaron inestabilidades fermiónicas en el capítulo 8. Se sabe que la descripción holográfica de una fase fermiónica homogénea explica algunas de las propiedades interesantes del metal extraño, como se explica en el capítulo 4. En particular, la función espectral resultante es compatible con una superficie de Fermi (FS) sin cuasi-partículas de larga vida media. Partiendo de este punto, se presenta un análisis de las inestabilidades de Pomeranchuk de la fase metálica extraña desde la perspectiva holográfica. 


\section{Teoría de Cuerdas y AdS/CFT}

\subsection{Teoría de cuerdas: nacimiento}

La teoría de cuerdas (ST) surge como una posible explicación de las interacciones fuertes, teoría que fue dejada de lado posteriormente y reemplazada por una QFT conocida como Cromodinámica Cuántica (QCD). QCD es una teoría de Yang-Mills con grupo de simetría de calibre o de "color" $S U(3)$ y tres colores de fermiones llamados quarks que transforman en la representación fundamental. Los estados observados a baja energía, conocidos genericamente como hadrones, son estados ligados de quarks, singletes de $S U(3)$; por ejemplo un mesón es una partícula constituida por un quark y un anti-quark. La interacción entre ambos es explicada si pensamos que existe una cuerda que conecta los dos quarks. Si $R$ es la longitud de la cuerda/separación entre quarks, para separar las partículas se necesita una energía proporcional a la longitud de la cuerda, $E=T R$, donde $T$ es la tension de la cuerda, relación que muestra porqué no es posible separar a los quarks (fenómeno conocido como confinamiento). Lo que ocurre cuando aumentamos la longitud de la cuerda es que la energía potencial se hace suficientemente grande como para crear otro par quark-antiquark, y la cuerda se convierte en dos cuerdas ya que resulta más favorable energéticamente; los extremos de las cuerdas siguen siendo quarks y antiquarks, no resultando quarks libres.

Como la tensión $T$ de la cuerda podría en principio reducirse a cero, es necesario que ésta oscile ya que el momento angular contribuye a la energía y entonces debe haber una relación entre el momento angular $J$ y la energía de la cuerda. Clásicamente el único parámetro con dimensiones que describe la cuerda es la tensión $T$. Mediante análisis dimensional y la definición de momento angular, se encuentra que:

$$
J \sim \frac{1}{T} M^{2}+\hbar
$$

Fenomenológicamente esta relación es escrita como:

$$
J \approx \alpha^{\prime} M^{2}+\alpha(0)
$$

donde $\alpha^{\prime} \sim 1 / T$, es la pendiente de Regge y $\alpha(0)$ las correcciones cuánticas. Hay muchas variedad de mesones, entre ellos los piones (mesones escalares), mesones vectoriales y mesones con mayor espín y energía. El espectro de mesones satisface con gran aproximación la relación (2) como se muestra en la figura (1), donde los valores de las constantes son: $\alpha^{\prime} \approx 0.9 \mathrm{GeV}^{-2}$ y $\alpha(0) \approx 0.5$. Por lo tanto vemos que los mesones son bien explicados mediante el modelo de cuerdas.

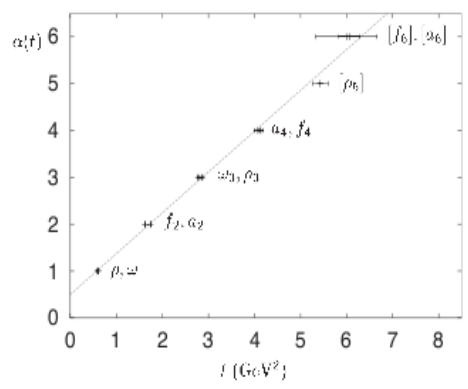

Figura 1: El eje horizontal representa la energía $M^{2}$ y el eje vertical representa los spins. Los mesones simbolizados con corchetes no son bien conocidos. La línea representa un ajuste usando la ecuación (2).

Como dijimos precedentemente, la teoría de interacciones fuertes actualmente aceptada es QCD. En QCD, un mesón es visto como un par quark-antiquark unido por gluones (las partículas de calibre de $S U(3)$ ). Las líneas de flujo de la electrodinámica se extienden por el espacio, en cambio las líneas de flujo de una teoría de color no se expanden sino más bien permanecen delgadas debido a las auto-interacciones entre los gluones

Si bien ST puede resultar una teoría consistente, si se pretende utilizarla se deben aceptar ciertas consecuencias:

- Para cuantizar ST consistentemente es necesario que el espacio-tiempo tenga 26 dimensiones si se quiere tener solo bosones en el espectro. Mientras que si se requiere que también contenga fermiones el número de dimensiones se reduce a 10 y la teoría debe ser supersimétrica. 
- Usando ST es posible explicar el confinamiento de quarks, pero QCD explica otros fenómenos además de éste. Por ejemplo, cuando la separación de los quarks se vuelve pequeña (en el ultravioleta) el tamaño del flujo no es despreciable y como consecuencia la energía potencial entre los dos quarks se comporta como $E \approx-1 / R$ (potencial de Coulomb); los modelos de cuerdas más simples no son capaces de explicar este comportamiento conocido como libertad asintótica.

Fue entre otras cosas debido a estos problemas que ST fue abandonada como teoría para describir la interacciones fuertes, pero surgió como una teoría candidata para unificar todas las interacciones, ya que como veremos ST incluye la gravedad.

\subsection{Cuerda bosónica}

ST tiene un solo parámetro con dimensiones que es la tensión, $T \equiv \frac{1}{2 \pi \alpha^{\prime}}=\frac{1}{\pi l_{s}}$; como $[T]=L^{-2},\left[\alpha^{\prime}\right]=L^{2}$. Por lo tanto introducimos el parámetro $l_{s} \equiv \sqrt{2 \alpha^{\prime}}$, la longitud de la cuerda. Esta es la escala fundamental donde la ST presenta sus mayores efectos, que es del orden de la longitud de Planck, $l_{s} \sim 10^{-35} \mathrm{~m}$; así macroscópicamente (a escalas mayores que $l_{s}$ ) la cuerda se ve como un punto.

En general podemos tener objetos extendidos en $p$ dimensiones llamados $p$-branas Una 0-brana es una partícula puntual, con acción $S_{0}=-m \int d s$, una 1 - brana es una cuerda y así siguiendo. La generalización de la acción de una partícula puntual a una p-brana en un espacio-tiempo $\mathrm{D}$ dimensional $(D \geq p)$ es:

$$
S_{p}=-T_{p} \int d \mu_{p}=-T_{p} \int \sqrt{-\operatorname{det}\left(G_{\alpha \beta}(\sigma)\right)} d^{p+1} \sigma
$$

donde $d \mu_{p}$ es el elemento de volumen $(\mathrm{p}+1)$ dimensional, definido por la métrica inducida $G_{\alpha, \beta}(\sigma)=$ $\frac{\partial X^{\mu}}{\partial \sigma^{\alpha}} \frac{\partial X^{\nu}}{\partial \sigma^{\beta}} g_{\mu \nu}(X)$ donde $\alpha, \beta=0,1, \ldots, p$ y $\sigma^{0}=\tau$, juega el rol de coordenada temporal $\sigma^{1}, \sigma^{2}, \ldots, \sigma^{p}$ son coordendas tipo espacio. La métrica $G_{\alpha, \beta}(\sigma)$ surge debido al inmersion de la $p$-brana en un espacio-tiempo D dimensional, es decir, $G_{\alpha, \beta}(\sigma)$ mide distancias sobre el volumen de mundo mientras que $g_{\mu \nu}(X)$ mide distancias en espacio-tiempo. Nos centraremos ahora en el caso de la acción para una cuerda, $p=1$.

La hoja de mundo (WS) de una cuerda será parametrizada por dos parámetros independientes $\left(\sigma^{0}, \sigma^{1}\right)=$ $(\tau, \sigma)$, con $\tau \in \mathbb{R}, \sigma \in[0, \pi]$. La inmersión de la cuerda en un espacio-tiempo D dimensional es definida por los campos $X^{\mu}(\tau, \sigma)$, donde $\mu=0,1, \ldots D-1$. Trabajar con la acción (3) resulta complicado debido a la presencia de la raíz cuadrada. Para evitar esto, Alexander Polyakov propuso otra acción que a nivel clásico resulta ser equivalente a (3):

$$
S=-\frac{T}{2} \int d \tau d \sigma \sqrt{-h} h^{\alpha \beta} \partial_{\alpha} X^{\mu} \partial_{\beta} X^{\nu} g_{\mu \nu}(X)
$$

donde $h_{\alpha \beta}(\tau, \sigma)$ es un nuevo campo auxiliar simétrico que representa la métrica en la WS.

Una vez conocida la acción es de fundamental importancia conocer sus simetrías. En espacio de Minkowski (4) presenta las siguientes simetrías:

Simetrías globales (Poincaré)

- Simetría de Lorentz: $X^{\mu} \rightarrow \Lambda_{\nu}^{\mu} X^{\nu} \quad, \quad \Lambda_{\mu}^{\rho} \Lambda_{\nu}^{\delta} \eta_{\rho \delta}=\eta_{\mu \nu}$

- Simetría frente a Traslaciones: $X^{\mu} \rightarrow X^{\mu}+\epsilon^{\mu}$

$\underline{\text { Simetrías locales }}$

- Simetría frente a difeomorfismos: $\sigma^{\alpha} \rightarrow \tilde{\sigma}^{\alpha}(\tau, \sigma)$

- Simetría de Weyl: $h_{\alpha \beta} \rightarrow \tilde{h}_{\alpha \beta}=\Lambda(\tau, \sigma) h_{\alpha \beta}$

Es posible utilizar las simetrías locales para fijar el calibre: $h_{\alpha \beta}=\eta_{\alpha \beta}$, por lo que se suele decir que la métrica $h_{\alpha \beta}$ no tiene dinámica. Utilizando este fijado de calibre la acción (4) se simplifica notablemente:

$$
S=\int d \tau d \sigma \mathcal{L}=\frac{T}{2} \int d \tau d \sigma\left((\dot{X})^{2}-\left(X^{\prime}\right)^{2}\right)
$$

De la definición del tensor de energía-impulso:

$$
T_{\alpha \beta} \equiv-\frac{2}{T} \frac{1}{\sqrt{-h}} \frac{\delta S}{\delta h^{\alpha \beta}}
$$


y del principio variacional se obtienen las ecuaciones de movimiento para $h_{\alpha \beta}$ :

$$
T_{\alpha \beta}=\partial_{\alpha} X \cdot \partial_{\beta} X-\frac{1}{2} h_{\alpha \beta} h^{\gamma \delta} \partial_{\gamma} X \cdot \partial_{\delta} X=0
$$

que en el calibre fijado da lugar a los siguientes vinculos:

$$
0=T_{00}=T_{11}=\frac{1}{2}\left(\dot{X}^{2}+X^{2}\right) \quad ; \quad T_{01}=T_{10}=\dot{X} \cdot X^{\prime}=0
$$

Por otro lado, al variar la acción de Polyakov respecto de los campos con las condiciones: $\delta X^{\mu}\left(\tau_{f}\right)=$ $\delta X^{\mu}\left(\tau_{i}\right)=0$, se obtiene

$$
\delta S=T \int d \sigma d \tau\left(X^{\prime \prime \mu}-\ddot{X}^{\mu}\right) \delta X_{\mu}-\left.T \int d \tau X^{\prime \mu} \delta X_{\mu}\right|_{\sigma=\pi}+\left.T \int d \tau X^{\prime \mu} \delta X_{\mu}\right|_{\sigma=0}
$$

La condición $\delta S=0$ implica la siguiente ecuación de movimiento para los campos $X^{\mu}$ :

$$
\left(\partial_{\tau}^{2}-\partial_{\sigma}^{2}\right) X^{\mu}(\tau, \sigma)=0
$$

Pero además los términos de borde también deben anularse. Hay varias formas de imponer condiciones de contorno (bc), que conducen a la posibilidad de tener cuerdas abiertas y cerradas.

Cuerdas cerradas: Si pensamos en cuerdas cerradas es natural pensar en bc periódicas:

$$
X^{\mu}(\tau, \sigma+\pi)=X^{\mu}(\tau, \sigma) \longrightarrow \delta X^{\mu}(\tau, \pi)=\delta X^{\mu}(\tau, 0)
$$

Por lo tanto los últimos dos términos de borde en (9) se cancelan entre si.

Cuerdas abiertas: Para cuerdas abiertas tenemos dos posibles alternativas para poder anular los términos de borde.

Condiciones de contorno de Neumann (NN): están definidas por:

$$
\partial_{\sigma} X^{\mu}(\tau, 0)=\partial_{\sigma} X^{\mu}(\tau, \pi)=0
$$
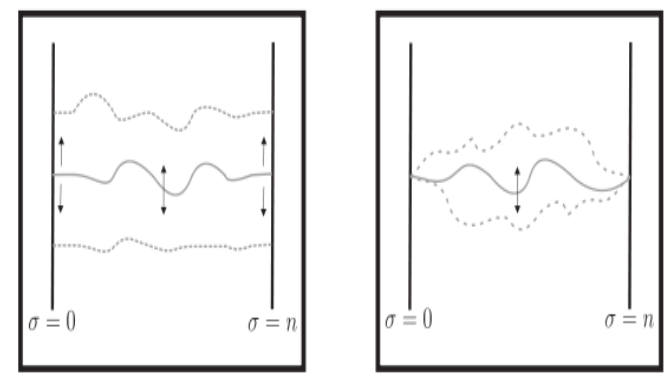

Figura 2: Figura izquierda: Condiciones de contorno de Neumann: La cuerda puede oscilar y sus puntos finales pueden moverse a lo largo del borde tanto como desaparezcan en ellas. Figura de la derecha:Condiciones de contorno de Dirichlet: La cuerda puede oscilar pero sus puntos finales estan fijo en el borde

Condiciones de contorno de Dirichlet (DD): corresponde a fijar un valor constante de los campos en los extremos de la cuerda:

$$
X^{\mu}(\tau, 0)=x_{0}^{\mu} \quad ; \quad X^{\mu}(\tau, \pi)=x_{\pi}^{\mu}
$$

La interpretación gráfica de estas bc se muestran en la figura $2^{1}$.

Una vez que contamos con la ecuación de movimiento de los campos (10) y las bc correspondientes a cuerdas cerradas y abiertas podemos pasar a resolverlas.

\footnotetext{
${ }^{1} \mathrm{Si}$ bien condiciones de contorno mixtas del tipo ND y DN son importantes, no serán de relevancia en esta Tesis y por lo tanto no las consideraremos.
} 
- Cuerda Cerrada: bc periódicas

$$
X^{\mu}(\tau, \sigma)=x^{\mu}+2 \alpha^{\prime} \tau p^{\mu}+\frac{i}{2} \sqrt{2 \alpha^{\prime}} \sum_{n \neq 0} \frac{1}{n}\left(\alpha_{n}^{\mu} e^{-2 i n(\tau-\sigma)}+\tilde{\alpha}_{n}^{\mu} e^{-2 i n(\tau+\sigma)}\right)
$$

donde la constante $x^{\mu}$ es el centro de masa y $p^{\mu}$ es el momento de la cuerda definido por:

$$
p^{\mu} \equiv \int_{0}^{\pi} d \sigma P^{\tau \mu}(\tau, \sigma) \quad ; \quad P^{\tau \mu} \equiv \frac{\partial \mathcal{L}}{\partial \dot{X}^{\mu}}=T \dot{X}^{\mu}
$$

Los dos primeros términos de (14) describen el movimiento del centro de masa de la cuerda, mientras que el resto describe las oscilaciones correspondientes a los modos $\alpha$ y $\tilde{\alpha}$

- Cuerda Abierta: bc NN

$$
X^{\mu}(\tau, \sigma)=x_{0}^{\mu}+\sqrt{2 \alpha^{\prime}} \alpha_{0}^{\mu} \tau+i \sqrt{2 \alpha^{\prime}} \sum_{m \neq 0} \frac{1}{m} \alpha_{m}^{\mu} e^{-i m \tau} \cos (m \sigma)
$$

- Cuerda Abierta: bc DD

$$
X^{\mu}(\tau, \sigma)=x_{0}^{\mu}+\frac{\sigma}{\pi}\left(x_{\pi}^{\mu}-x_{0}^{\mu}\right)+\sum_{m \neq 0} \frac{1}{m} \alpha_{m}^{\mu} e^{-i m \tau} \sin (m \sigma)
$$

Una vez encontradas las soluciones resulta de interés calcular observables físicos como por ejemplo el Hamiltoniano y la masa de la cuerda.

El Hamiltoniano es definido por:

$$
H=\int_{0}^{\pi} d \sigma\left(X_{\mu} P^{\tau \mu}-\mathcal{L}\right)
$$

Con $P^{\tau \mu}$ y $\mathcal{L}$ definidos en (15) y (5) respectivamente, obtenemos:

$$
H=\frac{T}{2} \int_{0}^{\pi} d \sigma\left(\dot{X}^{2}+X^{\prime 2}\right)
$$

Esta expresión nos permite obtener el Hamiltoniano de cuerdas cerradas y abiertas reemplazando las soluciones correspondientes.

Cuerdas Cerradas:

$$
H=\frac{1}{2} \sum_{n=-\infty}^{\infty}\left(\alpha_{-n} \cdot \alpha_{n}+\tilde{\alpha}_{-n} \cdot \tilde{\alpha}_{n}\right) \quad ; \quad \alpha_{0}^{\mu}=\tilde{\alpha}_{0}^{\mu}=\sqrt{\frac{\alpha^{\prime}}{2}} p^{\mu}
$$

Cuerdas Abiertas:

$$
H=\frac{1}{2} \sum_{n=-\infty}^{\infty} \alpha_{-n} \cdot \alpha_{n} \quad ; \quad \alpha_{0}^{\mu}=l_{s} p^{\mu} .
$$

Otro de los observables de interés (que en particular es igual a cero pero que nos da restricciones sobre las soluciones) es el tensor de energía-impulso. Resulta conveniente, como veremos, usar la expresión para $T_{\alpha \beta}$ en las coordenadas de cono de luz $\sigma^{ \pm} \equiv \tau \pm \sigma$; de (8) obtenemos:

$$
T_{--}=\left(\partial_{-} X^{\mu}\right)^{2} \quad ; \quad T_{++}=\left(\partial_{+} X^{\mu}\right)^{2} \quad ; \quad T_{+-}=T_{-+}=0
$$

Reemplazando la solución explícita para la cuerda cerrada llegamos a:

$$
T_{--}=2 l_{s}^{2} \sum_{m=-\infty}^{\infty} L_{m} e^{-2 m i(\tau-\sigma)} \quad ; \quad L_{m}=\frac{1}{2} \sum_{n=-\infty}^{\infty} \alpha_{m-n} \cdot \alpha_{n}
$$

Análogamente $T_{++}$está dado por:

$$
T_{++}=2 l_{s}^{2} \sum_{m=-\infty}^{\infty} \tilde{L}_{m} e^{-2 m i(\tau+\sigma)} \quad ; \quad \tilde{L}_{m}=\frac{1}{2} \sum_{n=-\infty}^{\infty} \tilde{\alpha}_{m-n} \cdot \tilde{\alpha}_{n}
$$

Recordemos que hasta ahora todas estas expresiones son clásicas y deben ser modificadas en el caso cuántico. De acuerdo a las definiciones de $L_{m}$ y $\tilde{L}_{m}$ podemos escribir el Hamiltoniano para cuerdas abiertas y cerradas: 
- Cuerda Cerrada

$$
H=2\left(L_{0}+\tilde{L}_{0}\right)
$$

- Cuerda Abierta

$$
H=L_{0}
$$

Una vez encontrado el Hamiltoniano y el tensor de energía-impulso en términos de los modos, podemos calcular la masa de la cuerda (abierta y cerrada). Para derivar ésta, partimos de la relación de masa-energía relativista:

$$
M^{2}=-p^{\mu} p_{\mu}
$$

Usando la definición de $p^{\mu}$ tanto para la cuerda abierta como para la cerrada dadas en (20) y (21) y usando los vínculos $L_{0}=0$ (cuerda abierta) y $L_{0}=\tilde{L}_{0}=0$ se obtiene

$$
M^{2}= \begin{cases}\frac{2}{\alpha^{\prime}} \sum_{n=1}^{\infty}\left(\alpha_{-n} \cdot \alpha_{n}+\tilde{\alpha}_{-n} \cdot \tilde{\alpha}_{n}\right) & \text { cuerdacerrada } \\ \frac{1}{\alpha^{\prime}} \sum_{n=1}^{\infty} \alpha_{-n} \cdot \alpha_{n} & \text { cuerdaabierta }\end{cases}
$$

\subsection{Espectro de la cuerda}

No explicaremos en detalle el procedimiento de cuantización, ver [15] por ejemplo. Cuando cuantizamos el campo $X^{\mu}$ los modos pasan a ser operadores que satisfacen:

$$
\left[\alpha_{n}^{\mu}, \alpha_{m}^{\nu}\right]=n \eta^{\mu \nu} \delta_{m+n, 0} \quad ; \quad\left[\tilde{\alpha}_{n}^{\mu}, \tilde{\alpha}_{m}^{\nu}\right]=n \eta^{\mu \nu} \delta_{m+n, 0}
$$

Esto afecta la definición de (23) y (24). Recordemos que clásicamente: $T_{\alpha \beta}=0$, pero cuando cuantizamos en general no es consistente imponer esta condición operatorialmente, lo que se puede imponer es $\left\langle\phi\left|\hat{T}_{\alpha \beta}\right| \phi\right\rangle$ para todo estado físico $|\phi\rangle$. Ya que ahora tenemos un orden normal derivado de la construcción del espacio de Fock asociados a estos osciladores los operadores $\hat{L}_{0}$ y $\tilde{\hat{L}}_{0}$ (que son los únicos que tienen problemas de ordenamiento normal) estarán definidos a menos de una constante arbitraria, y por lo tanto sobre estados físicos tendremos que:

$$
L_{0} \equiv \frac{1}{2} \alpha_{0}^{2}+\sum_{n=1}^{\infty} \alpha_{-n} \alpha_{n}=a \quad ; \quad \tilde{L}_{0} \equiv \frac{1}{2} \tilde{\alpha}_{0}^{2}+\sum_{n=1}^{\infty} \tilde{\alpha}_{-n} \tilde{\alpha}_{n}=\tilde{a}
$$

Para que la cuantizacion de la ST sea consistente es necesario imponer que $a=\tilde{a}=1, D=26$, ver [15].

Cuerda abierta

Consideramos bc NN en todas las coordenadas. De (30) imponiendo $L_{0}=1$ obtenemos:

$$
M^{2}=\frac{1}{\alpha^{\prime}}(N-1) \quad ; \quad N=\sum_{n=1}^{\infty} \alpha_{-n} \cdot \alpha_{n}
$$

donde $N$ es el operador de número. Recordemos que $\operatorname{los} \alpha_{-n}$ crean estados de modo $n>0$ mientras que $\operatorname{los} \alpha_{n}$ destruyen estados de modo $n>0$. Para describir los estados es conveniente utilizar el formalismo de cuantización de cono de luz donde se trabajan con los grados de libertad físicos que resultan ser los transversales, $X^{I}$ con $I=2, \ldots, D-1$.

- El estado fundamental en este formalismo es definido por

$$
\alpha_{n}^{I}\left|p^{+}, \vec{p}_{T}\right\rangle=0 \quad, \quad n \geq 1
$$

donde $p^{ \pm} \equiv p^{0} \pm p^{D-1} \quad$ y $\quad \vec{p}_{T}=\left(p^{2}, . ., p^{D-1}\right)^{t}$. La masa de este estado es $M^{2}=-1 / \alpha^{\prime}$ y no tiene osciladores presentes. Resulta ser un estado de masa negativa, comúnmente llamado estado taquiónico. La función de onda asociada a este estado se corresponde con un campo escalar. El signo $M^{2}<0$ da cuenta de una inestabilidad de la teoría.

- Primer estado excitado: $\alpha_{-1}^{I}\left|p^{+}, \vec{p}_{T}\right\rangle$ creamos un estado con modo $n=1$ por lo tanto existen 24 estados físicos no masivos. El estado mas general posible es una combinación líneal de los mismos:

$$
\sum_{I=2}^{D-1=25} \xi_{I} \alpha_{-1}^{I}\left|p^{+}, \vec{p}_{T}\right\rangle
$$

Esta expresión nos dice que el primer estado excitado de cuerdas abiertas corresponde a un campo vectorial sin masa, o sea, un fotón. No mencionaremos el resto de los estados excitados ya que no serán de interés. 
Cuerda cerrada

De (30) imponiendo $L_{0}+\tilde{L}_{0}=2$ obtenemos:

$$
\frac{1}{2} \alpha^{\prime} M^{2}=N+\tilde{N}-2
$$

- Estado fundamental: $M^{2}=-4 / \alpha^{\prime}$. Vemos que el estado fundamental de cuerdas cerradas son también estados taquiónicos con la diferencia que en el caso de cuerdas cerradas son más masivos en relación a la cuerda abierta.

- Los próximos estados excitados pueden ser construidos con dos osciladores actuando sobre el estado fundamental, teniendo en cuenta de (30) el vínculo $L_{0}-\tilde{L}_{0}=0$ o lo que es lo mismo $N=\tilde{N}$, por lo que resulta: $\alpha_{-1}^{I, \dagger} \tilde{\alpha}_{-1}^{J, \dagger}\left|p^{+}, \vec{p}_{T}\right\rangle$ con $I, J=2, \ldots, 25$. La masa del estado resulta ser $M^{2}=0$. Al estado general lo podemos escribir como:

$$
\sum_{I, J=2}^{D} R_{I J} \hat{\alpha}_{-1}^{I, \dagger} \hat{\tilde{\alpha}}_{-1}^{J, \dagger}\left|p^{+}, \vec{p}_{T}\right\rangle
$$

Cualquier matriz $R_{I J}$ siempre se puede escribir como $R_{I J}=h_{I J}+R / D \delta_{I J}+B_{I J}$ donde $h_{I J}$ es una matriz simétrica y con traza nula, $B_{I J}$ una matriz antisimétrica y $R / D \delta_{I J}$ la parte de traza $\left(R=R_{I}^{I}\right)$ lo que nos lleva a:

$$
\sum_{I, J} h_{I J} \hat{\alpha}_{-1}^{I, \dagger} \hat{\tilde{\alpha}}_{-1}^{J, \dagger}\left|p^{+}, \vec{p}_{T}\right\rangle+\sum_{I} \frac{R}{D} \hat{\alpha}_{-1}^{I, \dagger} \hat{\tilde{\alpha}}_{-1}^{I, \dagger}\left|p^{+}, \vec{p}_{T}\right\rangle+\sum_{I, J} B_{I J} \hat{\alpha}_{-1}^{I, \dagger} \hat{\tilde{\alpha}}_{-1}^{J, \dagger}\left|p^{+}, \vec{p}_{T}\right\rangle
$$

Existe una relación uno a uno entre entre el primer término de (36) con los estados cuánticos que surgen tras cuantizar pequeñas perturbaciones $h_{\mu \nu}$ alrededor del espacio de Minkowski usando la gravedad de Einstein. Por lo tanto estos estados son identificados con gravitones. El segundo término de la ecuación (36) se corresponde con un estado sin índices que se asocia naturalmente a una partícula escalar sin masa, el dilatón. El tercer término de (36) se corresponde con estados de una partícula asociados a un campo tensorial anti-simétrico $B_{\mu \nu}$ denominado campo de Kalb-Ramond.

\subsection{D-branas}

Las Dp-Branas surgen de las b.c de la ST. Estas resultan ser objetos de $p$ dimensiones donde los extremos de las cuerdas abiertas pueden ser anclados. Consideremos una cuerda entre dos $D p-$ branas planas paralelas en espacio de Minkowski $D$ dimensional, con direcciones tangentes $(0,1, I), I=$ $2,3, \ldots, p$ y perpendiculares $i=p+1, \ldots, D-1$. Trabajando siempre en la cuantización en el cono de luz, el espectro de masa viene dado por:

$$
M^{2}=\frac{N-1}{\alpha^{\prime}}+T^{2}\left(x_{a}^{j}-x_{b}^{j}\right)^{2} \quad ; \quad N=\sum_{n=1}^{\infty} \alpha_{-n}^{I} \alpha_{n}^{I}+\sum_{n=1}^{\infty} \alpha_{-n}^{j} \alpha_{n}^{j}
$$

donde $a, b=1,2$ etiquetan las branas. Analizaremos primero

Caso $a=b$, una Dp-brana.

- Estado fundamental: Encontramos estados taquiónicos con $M^{2}=-1 / \alpha^{\prime}$ análogos a los que aparecen en (32).

- Estado excitado: Tenemos dos tipo de osciladores que pueden actuar sobre el estado fundamental. Consideremos primero los osciladores que tienen un índice asociado a la coordenada tangente a la brana $I$. Estos estados son de la forma: $\alpha_{-1}^{I}\left|p^{+}, \vec{p}\right\rangle$ y tienen $M^{2}=0$. Tenemos un número total de estados sin masa igual a $(p+1)-2$. Vemos que coincide con el número de grados de libertad de un campo de Maxwell en $p+1$ dimensiones, con lo cual podemos concluir que:

Una Dp-brana tiene un campo de calibre U(1) viviendo sobre su volumen de mundo.

- Ahora consideremos osciladores que tienen un índice normal a la brana. Los estados son de la forma: $\alpha_{-1}^{j}\left|p^{+}, \vec{p}\right\rangle$ y tienen $M^{2}=0$. Ya que $j$ no es un índice de Lorentz para la brana, tenemos entonces un campo escalar no masivo por cada dirección normal a la brana. Concluimos que: 
Una Dp-brana tiene $D-p-1$ campos escalares no masivos, uno por cada dirección normal a la brana, viviendo sobre su volumen de mundo.

Caso: $x_{a}^{i} \neq x_{b}^{i}$ - análisis general. Supongamos que tenemos $2 \mathrm{Dp}$-branas. Los diferentes sectores son

etiquetados por $[a b]$ donde $a, b=1,2$. El primer índice denota la brana sobre la cual el extremo $\sigma=0$ de la cuerda se encuentra, mientras que el segundo índice denota la brana sobre la cual el extremo $\sigma=\pi$ se encuentra. Así los estados base son de la forma:

$$
\text { osciladores }\left|[a, b] p^{+}, \vec{p}\right\rangle
$$

Si las dos Dp-branas coinciden tendremos ahora un total de cuatro campo vectoriales no masivos, uno por cada sector, que forman un multiplete vectorial de una teoría de calibre $U(2)$. Si las branas están separadas entonces, de acuerdo con (37) los sectores no diagonales serán masivos (tendremos bosones vectoriales masivos) y la simetría del grupo de calibre se rompe a $U(1) \times U(1)$.

En el caso más general de tener $N$ Dp-branas paralelas, tendremos un total de $N \times N=N^{2}$ sectores etiquetados por $[a b]$ donde $a, b=1, \ldots, N$. Si las N Dp-Branas son coincidentes los $N^{2}$ sectores resultan en $N^{2}$ campos de calibre no masivos, junto con $D-p-1$ campos escalares viviendo en la adjunta de $U(N)$ $\left(N^{2}\right.$ en total). Esto define una teoría de Yang-Mills $U(N)$ sobre el volumen de mundo de las $N$ Dp-branas coincidentes. Concluimos que:

Sobre $N$ Dp-Branas coincidentes viven campos de calibre no masivos junto con $D-p-1$ escalares en la adjunta de $U(N)$.

Notamos por completitud que en el caso de tener grupos de branas separadas entre si, se dispara un mecanismo de Higgs por el cual algunos campos de calibre adquieren masa dando lugar a distintos patrones de ruptura de simetría.

\subsection{Interacciones de cuerdas}

Como sabemos una partícula dibuja una línea de mundo cuando se propaga por el espacio-tiempo, en cambio una cuerda deja a su paso una superficie u hoja de mundo (WS). En la figura (3) de la izquierda, se puede apreciar un diagrama de Feynman para una interacción entre partículas. Este diagrama se ve reemplazado para cuerdas cerradas en la figura de la derecha.

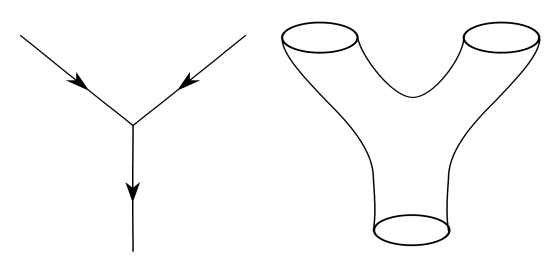

Figura 3: La figura de la izquierda representa un diagrama de Feynman asociado a un vértice de interacción de tres puntos (partículas puntuales). El diagrama de interacción ánalogo si se considera a las partículas como cuerdas cerradas se muestra en la figura de la derecha.

El objeto básico a computar en ST a nivel perturbativo es la matriz-S (ver ecuación (41)), o sea, las amplitudes de colisión entre estados asintóticos in y out que representan partículas. Por ejemplo, la colisión entre dos estados in y dos estados out es descripto en términos de la WS de las cuerdas como se muestra en la figura (4). 


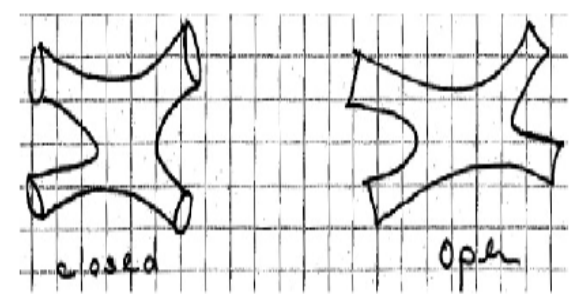

Figura 4: La figura de la izquierda representa un scattering entre dos cuerdas cerradas. La figura de la derecha también es un scattering pero entre cuerdas abiertas.

De la misma podemos apreciar la ausencia de vértices interactuantes locales definidos. También vemos que luce localmente como la WS de una cuerda propagándose libremente y solo las propiedades globales capturan la interacción. Otro aspecto fundamental en ST es que las interacciones entre cuerdas ya se encuentran codificadas en la teoría, no es necesario agregar términos extras (solo términos topológicos como veremos a continuación). Las interacciones se pueden ver de las distintas formas que puede tomar la hoja de mundo.
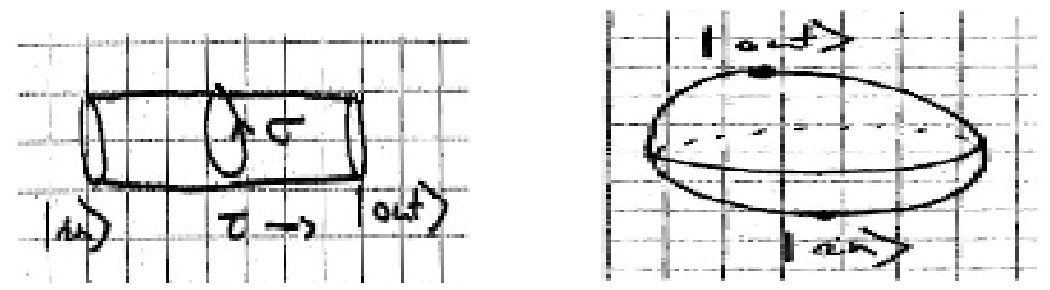

Figura 5: A la izquierda se muestra la WS que representa el propagador de una cuerda cerrada, topológicamente un cilindro. A la derecha, la WS es mapeada conformemente a una $S^{2}$ con dos punturas.

Los estados in y out se encuentran localizados en el infinito, es decir las patas de los diagramas de la figura (3) se extienden al infinito. Es posible mapear tales estados en el infinito a puntos (inserciones) sobre la hoja de mundo, vía una transformación conforme (recordemos que la acción de Polyakov una vez fijadas las simetrías locales presenta invarianza conforme). Por ejemplo, consideramos la WS que describe la amplitud uno 1 a 1 ó propagador de cuerdas cerradas. Comenzamos con una superficie 2-dimensional con coordenadas $(\tau, \sigma)$ y métrica euclídea: $d s^{2}=d \tau^{2}+d \sigma^{2}$. Para cuerdas cerradas tomaremos $\sigma$ periódica (como especificamos en la sección (2.2)), $\sigma \sim \sigma+2 \pi$, por lo cual tenemos topológicamente un cilindro (como se muestra en la figura (5) de la izquierda), con secciones espaciales a $\tau=\mp \infty$ correspondientes a los estados in/out. Luego el cambio de coordenadas $w / \bar{w}=\tau \pm i \sigma$ nos lleva a $d s^{2}=d w d \bar{w}$, y haciendo la transformación conforme $z / \bar{z}=e^{w} / e^{\bar{w}}$ mapeamos el cilindro a una esfera, con los estados $\mid$ in $\rangle$ y $\mid$ out $\rangle$ insertados en los polos de la esfera (como se muestra en la figura (5) de la derecha). De este ejemplo se desprende un resultado más general (ver figura (6)): siempre podemos pensar las interacciones, vía un mapeo conforme, como una esfera con inserciones donde cada inserción se corresponde con un operador local $W(\tau, \sigma)$ asociado a un estado colisionante. Tales operadores pueden ser construídos para cada estado de la cuerda y se denominan operadores de vértice. ${ }^{2}$

\footnotetext{
${ }^{2}$ Estrictamente hablando esto es cierto para estados on-shell, invariantes conformes.
} 


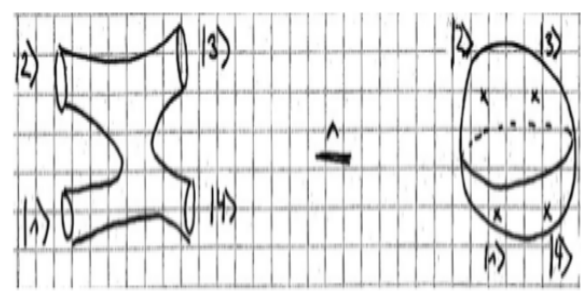

Figura 6: Se muestra gráficamente que siempre es posible mapear vía una transformación conforme la colisión entre dos cuerdas y llevarla a una esfera con 4 inserciones en ella, correspondientes a los cuatro estados presentes. Las inserciones se pueden encontrar en cualquier punto de la esfera. En el caso del mismo proceso pero para cuerdas abiertas el mapeo también es posible pero las inserciones correspondientes a los cuatro estados deben vivir en el borde de la WS.

A este punto debemos tener presente que las WS pueden tener diferentes topologías. Podemos tomar un world-sheet e ir agregando cualquier número de manijas, que se corresponden con los lazos en los diagramas de Feynman de QFT. El número de manijas presentes es llamado el genus g de la WS. Es posible extender la acción de Polyakov manteniendo las mismas simetrías y teniendo en cuenta la topología de la hoja de mundo:

$$
S=-\frac{T}{2} \int_{\Sigma} d^{2} \sigma \sqrt{h} h^{\alpha \beta} \partial_{\alpha} X^{\mu} \partial_{\beta} X^{\nu} G_{\mu \nu}(X)+\frac{\phi_{0}}{4 \pi} \int_{\partial \Sigma} d^{2} \sigma \sqrt{h} R^{(2)}
$$

donde $\phi_{0}$ es una constante y $R^{(2)}$ es el escalar de Ricci de $h_{\alpha \beta}$. Sin embargo el nuevo término es esencialmente un invariante topológico que puede ser construido para cualquier superficie orientable (llamada superficie de Riemann), la característica de Euler:

$$
\chi=2-2 g-b=\frac{1}{4 \pi} \int_{\partial \Sigma} d^{2} \sigma \sqrt{h} R^{(2)}
$$

donde $g$ es el genus de la superficie y $b$ el número de fronteras. Vemos entonces que el término extra en (39) resulta ser $\phi_{0} \chi$; el mismo lleva un registro de la topología de la WS en la integral de camino.

$$
S_{j_{1}} \ldots j_{b}\left(k_{1} \ldots k_{b}\right)=\sum_{\text {Topologias }} \int \frac{D X D h}{V o l_{\text {diff } \times W e y l}} e^{-S_{P}-\phi_{0} \chi} \prod_{i=1}^{b} V_{j_{i}}\left(k_{i}\right)
$$

donde $V_{j_{i}}\left(k_{i}\right)=\int W_{j_{i}}\left(\tau, \sigma ; k_{i}\right)$ es el operador de vértice (integrado a la superficie) del estado $j_{i}$ que representa la partícula $i$ con momento $k_{i}$. Definamos $g_{s} \equiv e^{\phi_{0}}$; vemos en (41) que un término de la forma:

$$
e^{-\phi_{0} \chi}=g_{s}^{-2+2 g+b}
$$

está presente. Para $g_{s}<<1 \Rightarrow$ la serie topológica define una serie perturbativa.

Consideremos el caso de una cuerda cerrada. Añadir una manija a la WS corresponde añadir un agujero, o sea pasar de $g$ a $g+1$ y así el número de Euler pasa de: $-\chi \rightarrow-\chi+2$. Físicamente añadir una manija describe la emisión y absorción internas de cuerdas cerradas. Si pensamos en términos de diagramas de Feynman esto corresponde a pensar en dos vértices cada uno con una constante de acoplamiento. Por otro lado agregar un estado externo hace que $b$ pase a $b+1$ y entonces $-\chi$ pasa $a-\chi \rightarrow \chi+1$; en términos de diagramas de Feynman se agrega un vértice con su constante de acoplamiento. Teniendo en cuenta (42), estos dos hechos nos llevan a interpretar a $g_{s}$ como la constante de acoplamiento de la cuerda cerrada:

$$
g_{\text {closed }}=e^{\phi_{0}}=g_{s}
$$

Un análisis similar en el caso de la cuerda abierta nos lleva a concluir:

$$
g_{\text {open }}=e^{\frac{\phi_{0}}{2}}=g_{s}^{\frac{1}{2}}=g_{\text {closed }}^{\frac{1}{2}}
$$

En conclusión, de la serie perturbativa (41) se deduce que las interacciones entre cuerdas están determinadas por la suma sobre superficies bidimensionales con diferentes topologías, correspondiente a una expansión pertubativa gravitacional. Por ejemplo, en el caso de cuerdas cerradas, la esfera (orden árbol, $\left.g=0, g_{s}^{-2}\right)$ se corresponde a la gravedad clásica, el toro $\left(g=1, g_{s}^{2}\right)$ se corresponde a gravedad a 1-lazo y así siguiendo. Esto se muestra en la figura (7). 


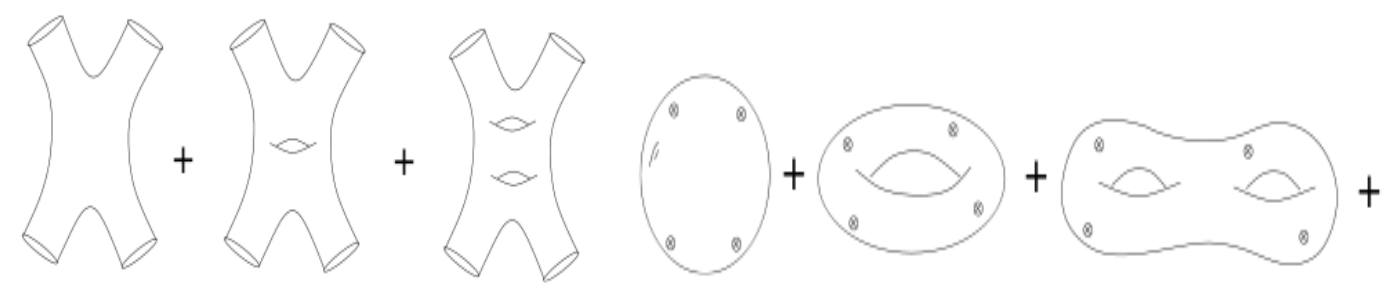

Figura 7: Se muestra la suma de procesos de colisión de cuatro estados externos con diferentes agujeros en la WS de la cuerda. Tal suma de diagramas es equivalente a una suma sobre superficies de Riemann cerradas con distintos genus y cuatro inserciones sobre su superficie.

Si $Z_{\text {string }}$ es la función de partición /amplitud, podemos escribir para la parte conexa:

$$
G_{\text {string }} \equiv \ln Z_{\text {string }}=\sum_{g=0}^{\infty} g_{s}^{2 g-2} G_{g}\left(l_{s}\right)
$$

donde hemos hecho explícita la dependencia $G_{g}\left(l_{s}\right)$, ya que la teoría tiene correcciones en $\alpha^{\prime} \sim l_{s}{ }^{2}$.

\subsection{Supergravedad}

Hemos visto que las oscilaciones más bajas de ST corresponden a partículas descriptas por teorías de calibre y la teoría de la gravedad (ver sección (2.3)). Pero dado que existen infinitos estados de oscilación de la cuerda obtenidos considerando los infinitos modos $n$, tenemos infinitas partículas predichas por ST, lo cual entra en conflicto con lo que sabemos de la naturaleza a las escalas de energías observadas hasta el momento en los experimentos. Ahora bien, notemos que para que las cuerdas oscilen esto demandara energía, este número infinito de armónicos o modos que podemos generar desde el estado fundamental corresponden a partículas masivas cuyas masas son típicamente del orden de $1 / l_{s}$, correspondiendo a partículas elementales extremadamente pesadas, por lo que las partículas que nos importan en un principio son las más livianas y finitas en número, como lo son las observadas al momento en la naturaleza.

Por otro lado, dado que la existencia de taquiónes en el espectro es una característica no física, se debieran considerar ST donde los mismos no están presentes. Es bien sabido que es posible eliminarlos si consideramos versiones supersimétricas de las mismas, las teorías de supercuerdas (SST). Ya que SST describe un número finito de partículas elementales cuando podemos ignorar los efectos de la longitud de la cuerda $l_{s}$, debiera ser descripta en términos de una teoría de campos. Tales teorías son conocidas como teorías de Supergravedad.

La forma de la acción de supergravedad puede ser derivada de principios generales. Es posible mostrar que una ST que contiene la gravedad debe respetar el principio de covarianza general, con lo cual el sector de la gravedad a orden más bajo en derivadas (ó en $l_{s}$ ) debe ser de la forma:

$$
S=\frac{1}{16 \pi G_{10}} \int d^{10} x \sqrt{-g} R
$$

como en Relatividad General. La escribimos en 10 dimensiones ya que la SST solo puede ser cuantizada en 10 dimensiones. Similarmente se puede mostrar que la acción asociada a campos vectoriales no masivos en ST debe tener invarianza de calibre, por lo que debe tomar la misma forma a como lo hace en teoría de campos en 4 dimensiones en fondos curvos.

Debemos ser capaces de relacionar la constante de acoplamiento de ST, $g_{s}$, con la de la gravitación $G_{10}$ y con la de la teoría de calibre $g_{Y M}$. Analicemos primero el caso de la gravitación. Haciendo un desarrollo de la métrica $g$ del espacio-tiempo alrededor de la métrica de Minskowski $g_{\mu \nu}=\eta_{\mu \nu}+h_{\mu \nu}$, obtenemos de (46):

$$
S=\frac{1}{16 \pi G_{10}} \int d^{10} x\left(\partial h \partial h+h \partial h \partial h+h^{2} \partial h \partial h+\ldots\right)
$$

De aquí podemos ver que la emisión de un gravitón es proporcional a $G_{10}^{1 / 2}$. Pero de (43) sabemos que la emisión de una cuerda cerrada (un gravitón) se corresponde con un $g_{s}$. Entonces concluimos que $G_{10} \propto g_{s}^{2}$. Ahora escribimos la acción para el campo de calibre

$$
S=\frac{1}{g_{Y M}^{2}} \int d^{p+1} x\left(\partial A \partial A+A^{2} \partial A+A^{4}\right)
$$


En este caso la emisión de un campo de calibre se corresponde con $g_{Y M}$, mientras que la emisión de una cuerda abierta se corresponde con $g_{s}{ }^{\frac{1}{2}}$ (ver ecuación (44)), por lo tanto vemos que $g_{Y M}^{2} \propto g_{s}$. Las constantes de proporcionalidad se pueden fijar haciendo análisis dimensional. De acuerdo a nuestra convención la acción es siempre adimensional entonces $\left[G_{10}\right]=L^{8}$ de $(47)$. Por otro lado, en ST solo hay una constante fundamental con unidades $l_{s}$, con lo cual la constante de proporcionalidad entre $G_{10}$ y $g_{s}^{2}$ debe ser $l_{s}^{8}$. De igual forma en el caso de la acción del campo de calibre $\left[g_{Y M}^{2}\right]=L^{p-2}$ (ya que $[A]=L^{-1}$ ) para que la acción sea adimensional, entonces la constante de proporcionalidad con unidades debe ser $l_{s}^{p-3}$. Resumiendo:

$$
G_{10} \simeq g_{s}^{2} l_{s}^{8} \quad ; \quad g_{Y M}^{2} \simeq g_{s} l_{s}^{p-3}
$$

La acción (46) es válida solo cuando se puede despreciar los efectos de la longitud de la cuerda $l_{s}$. Cuando la longitud de la cuerda no puede despreciarse entonces la acción contendrá un número infinito de términos en potencias de $R$ y sus derivadas correspondientes a correcciones a altas energías de la gravedad debidas a efectos cuánticos:

$$
S=\frac{1}{16 \pi G_{10}} \int d^{10} x \sqrt{-g}\left(R+l_{s}^{2} R^{2}+\ldots\right)
$$

Dichas correcciones en $\alpha^{\prime}$, pueden ser ignoradas cuando $\alpha^{\prime} R<<1$. Tales términos en la expansión topológica de la ST corresponde a los diagramas mas bajos de la esfera. Para ser más precisos (el sector bosónico de) la acción de supergravedad que describe los modos no masivos de la SST a nivel árbol (esfera) es:

$$
S=\frac{1}{16 \pi G_{10}} \int d^{10} x \sqrt{-g} e^{-2 \phi}\left(R+4(\nabla \phi)^{2}\right)-\frac{1}{12} H^{\mu \nu \rho} H_{\mu \nu \rho}+\ldots
$$

donde $\phi$ es el campo escalar asociado al dilatón y $H_{H_{\mu \nu \rho}} \equiv \partial_{\mu} B_{\nu \rho}+$ cíclicos es el tensor de calibre asociado al campo de Kalb-Ramond $B_{\mu n u}$. Notar la presencia del factor $e^{-2 \phi}$, que proviene de la identificación de ecuación (42)del valor de expectación del dilatón con la constante $\phi_{0}$ que define la constante de acoplamiento, $g_{s}^{-\chi}=g_{s}^{-2}$ en la esfera $(g=0)$.

\subsection{La correspondencia AdS/CFT}

\subsubsection{Principio holográfico}

Sabemos de la solución de agujero negro de Schwarzschild con horizonte en $r_{0}=2 M G / c^{2}$, que el área del mismo crece como $A=4 \pi r_{0}^{2}=16 M^{2} G^{2} / c^{4}$. Ya que la masa del BH (agujero negro) solo puede aumentar (nada puede salir de éste, solo pueden caer cosas hacia él) el área se incrementará siempre. Esto nos recuerda a una variable termodinámica, la entropía, que es una cantidad que siempre aumenta. Con lo cual podríamos suponer que $S \propto A$. Es decir que el BH obedece la segunda ley de la termodinámica. Pero no solo obedece la segunda ley sino que obedece todas las leyes de la termodinámica.

A lo largo de esta Tesis nos centraremos en BH estacionarios. En Relatividad General una solución de BH satisface los teoremas de no pelo, es decir está determinada solo por unos pocos parámetros: masa, carga y momento angular. Esto nos indica que no depende de la composición original de la estrella colapsante de la cual provino. La situación se asemeja a la teoría termodinámica donde no es necesario describir las posiciones y velocidades de las moléculas para caracterizar el sistema, solo son necesarias unas pocas variables macroscópicas como la presión y temperatura. Las leyes de los BH parecen ser muy similares a las leyes de la termodinámica (ver [18] para una descripción detallada). Ahora bien, sabemos que la entropía de un sistema estadístico resulta ser proporcional al volumen del mismo pero hemos supuesto que la entropía de un $\mathrm{BH}$ es proporcional al área, o sea, se comporta muy distinto. Podemos decir entonces que:

Primera clave de AdS/CFT: La entropía de un BH sugiere que éste puede ser descripto por un sistema estadístico cuya dimension espacial es una menos que la dimensión del sistema gravitacional.

Tal idea es llamada Principio Holográfico.

\subsubsection{Temperatura de Hawking y formalismo euclídeo}

Pasemos ahora a calcular la temperatura de un BH. Consideremos una métrica estática de la forma:

$$
d s^{2}=-f(r) d t^{2}+\frac{1}{f(r)} d r^{2}+\ldots
$$

Pasando a tiempo euclídeo $t=-i \tau$ :

$$
d s_{E}^{2}=+f(r) d \tau^{2}+\frac{1}{f(r)} d r^{2}+\ldots
$$


Nos enfocaremos en la región cerca del horizonte donde: $f\left(r_{0}\right)=0, r \approx r_{0} \rightarrow f(r) \approx f^{\prime}\left(r_{0}\right)\left(r-r_{0}\right)+\ldots$, con lo cual:

$$
d s_{E}^{2} \approx f^{\prime}\left(r_{0}\right)\left(r-r_{0}\right) d \tau^{2}+\frac{1}{f^{\prime}\left(r_{0}\right)\left(r-r_{0}\right)} d r^{2}+\ldots=d \rho^{2}+\rho^{2} d \phi^{2}
$$

donde introdujimos las coordenadas: $\rho \equiv 2 \sqrt{\left(r-r_{0}\right) / f^{\prime}\left(r_{0}\right)}, \phi \equiv f^{\prime}\left(r_{0}\right) \tau / 2$. Vemos que la región cercana al horizonte resulta el origen de un plano si imponemos ausencia de singularidad cónica, o sea, identificamos: $\phi \approx \phi+2 \pi$, con lo cual $f^{\prime}\left(r_{0}\right) \tau / 2 \sim f^{\prime}\left(r_{0}\right) \tau / 2+2 \pi$. Entonces $\tau \sim \tau+\beta=\tau+\frac{4 \pi}{f^{\prime}\left(r_{0}\right)}$, y ya que en mecánica estadística cuántica la periodicidad en el tiempo imaginario está asociada a la inversa de la temperatura, identificamos $\beta=1 / T$ y por lo tanto:

$$
T=\frac{f^{\prime}\left(r_{0}\right)}{4 \pi}
$$

Cabe mencionar que la métrica en coordenadas polares no cubre el interior del BH ya que $r=r_{0}$ es el origen del sistema de coordenadas polares.

Tentativamente una teoría de la gravedad cuántica está definida por la función de partición:

$$
Z=\int D g e^{i / \hbar S[g]}=\int D g e^{-S_{E}[g] / \hbar}
$$

donde introdujimos la acción euclídea: $S_{E} \equiv-\left.i S\right|_{t=-i \tau}$ Ya que una métrica euclidea de BH es periódica en el tiempo, es natural decir que (56) es la función de partición del BH análoga a las funciones de partición que aparecen en mecánica estadística cuántica. El problema surge en que no es claro cómo tratar con tal integral ya que presenta divergencias. De todas maneras semi-clásicamente la contribución más dominante viene de la solución clásica (la aproximación de punto silla/ método de la fase estacionaria). En esta aproximación:

$$
Z \approx e^{-\left.S_{E}\right|_{\text {on-shell }}}
$$

La energía libre es por definición: $F=-\ln Z / \beta$, con lo cual:

$$
\left.S_{E}\right|_{o n-s h e l l}=\beta F
$$

\subsubsection{Teorías de calibre de gran $N_{c}$}

QCD tiene un poder predictivo limitado, pues solo es posible analizar problemas en forma perturbativa. Un método de aproximación para estudiar interacciónes no perturbativas es considerar la teoría en el límite de gran número de colores $N_{c}$. Si bien QCD tiene tres colores se espera que la aproximación capture información relevante. Si consideramos una teoría de YM $U\left(N_{c}\right)$ donde los campos de calibre se escriben como matrices $N_{c} \times N_{c},\left(A_{\mu}\right)_{j}^{i}$, el número de grados de libertad será $N_{c}{ }^{2}$. Por lo que más generalmente se puede pensar este límite como un límite de gran número de grados de libertad.

Una teoría de calibre tiene dos parámetros libres: la constante de acoplamiento de la teoría $g_{Y M}$ y el número de colores $N_{c}$. En lugar de usar $g_{Y M}$ usaremos la llamada constante de 't Hooft: $\lambda \equiv g_{Y M}{ }^{2} N_{c}$. El límite de gran $N_{c}$ se define como:

$$
N_{c} \rightarrow \infty \quad ; \quad \lambda \text { fijo }
$$

Los diagramas de Feynmann en teorías de campos, por ejemplo los propagadores, son representados mediante líneas, pero en teorías de calibre para mantener la pista de los colores es conveniente usar la notación de doble línea: donde el propagador es representado por una doble línea con índices opuestos. A saber una línea representa la fila de la matriz $\left(A_{\mu}\right)_{j}^{i}\left(A_{\mu}=A_{\mu}^{a} T^{a}\right.$ y $T^{a}$ está en la representación adjunta de $U\left(N_{c}\right)$ mientras que la otra representa la columna. En la figura (8) se puede ver tal esquematización

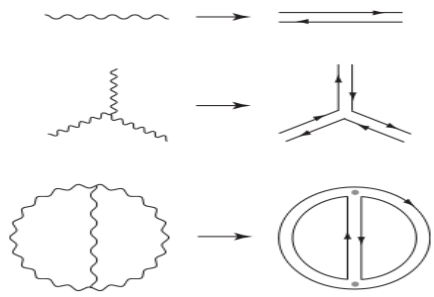

Figura 8: El primer gráfico representa el propagador del campo de calibre y su correspondiente diagrama de doble línea. El segundo representa un vértice de interacción y su diagrama de doble línea. El último esquema es un ejemplo de diagrama que contribuye a la amplitud de vacío. 
El Lagrangiano de una teoría de YM es esquemáticamente representado como:

$$
L=\frac{1}{g_{Y M^{2}}}\left((\partial A \partial A)+A^{2} \partial A+A^{4}\right)=\frac{N_{c}}{\lambda}(\ldots)
$$

Las reglas de Feynman asociadas a este Lagrangiano para calcular las amplitudes de dispersión son:

- Se asocia el factor $\frac{\lambda}{N_{c}}$ por cada propagador.

- Se asocia el factor $N_{c} / \lambda$ por cada vértice de interacción.

- Se asocia el factor $N_{c}$ por cada lazo.

Si V es el número de vértices, E el número de propagadores y $\mathrm{F}$ el número de lazos, entonces:

$$
\left(\frac{N_{c}}{\lambda}\right)^{V}\left(\frac{\lambda}{N_{c}}\right)^{E} N_{c}^{F}=\lambda^{E-V} N_{c}^{V-E+F}
$$

es el factor asociado al diagrama en cuestión.

(a)

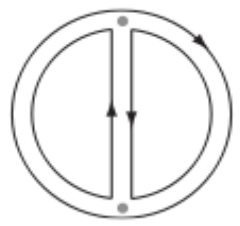

(b)

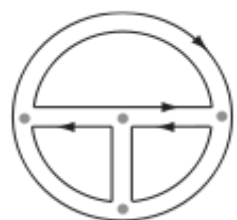

(c)

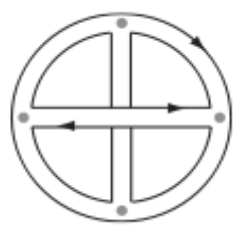

Figura 9: Ejemplos de distintos diagramas de amplitudes de vacío. Las figuras (a) y (b) son diagramas planares mientras que la figura (c) es un diagrama no-planar.

Por ejemplo el diagrama $(9)($ a) tiene $(V, E, F)=(2,3,3)$ entonces

$$
\left(\frac{N_{c}}{\lambda}\right)^{2}\left(\frac{\lambda}{N_{c}}\right)^{3} N_{c}^{3}=\lambda N_{c}^{2}
$$

mientras que el diagrama $(9)(\mathrm{b})$ tiene $(V, E, F)=(4,6,4)$ entonces

$$
\left(\frac{N_{c}}{\lambda}\right)^{4}\left(\frac{\lambda}{N_{c}}\right)^{6} N_{c}^{4}=\lambda^{2} N_{c}^{2}
$$

Para calcular la amplitud de dispersión de un proceso (amplitud de vacío en este caso) estos resultados son sumados:

$$
A=\left(a_{0}+a_{1} \lambda+a_{2} \lambda^{2}+\ldots\right) N_{c}^{2}=a(\lambda) N_{c}^{2}
$$

Pero no siempre se puede escribir de esta forma, por ejemplo el diagrama $(9)$ (c) tiene $(V, E, F)=(4,6,2)$ con lo cual

$$
\left(\frac{N_{c}}{\lambda}\right)^{4}\left(\frac{\lambda}{N_{c}}\right)^{6} N_{c}^{2}=\lambda^{2}
$$

Los diagramas como los de las figuras (9)(a) y (b) son llamados planares pues siempre pueden ser dibujados sobre esferas o en un plano, mientras que los diagramas como el de la figura (9)(c) son llamados no-planares. La amplitud se puede escribir:

$$
A=f_{0}(\lambda) N_{c}^{2}+f_{1}(\lambda) N_{c}^{0}+f_{2}(\lambda) \frac{1}{N_{c}^{2}}+\ldots
$$

En el límite de gran $N_{c}$, es el primer término de (66), o sea los diagramas planares, el que domina. En general se puede entender la relación que existe entre los diagramas de la figura (9) y la topología de la siguiente forma. Cada lazo en (9) se puede asociar con superficies como se muestra en la figura (10). 


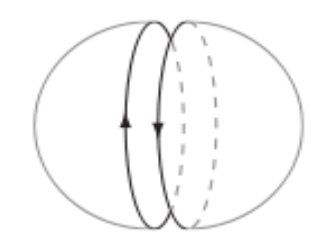

Figura 10: Representación que muestra la relación entre un diagrama y una superficie.

Cada propagador es un borde de la superficie y cada vértice es un vértice de la superficie. Entonces se puede caracterizar el número de propagadores $E$ como el número de bordes de la superficie, el número de lazos $F$ como el número de caras de la superficie y $V$ como el número de vértices de esta. Bajo esta interpretación, la potencia de $N_{c}$ en (61) coincide con la definición topológica de caracterśtica de Euler $\chi$ que definimos en la sección (2.5):

$$
\chi=V-E+F=2-2 g
$$

que para una esfera $(g=0) \quad \chi=2$ y para un toro $(g=1) \chi=0$. A saber, los diagramas (9)(a)(b) corresponden a la esfera, mientras que el diagrama $(9)(\mathrm{c})$ se corresponde con el toro. Esta comparación se muestra en la figura (11)

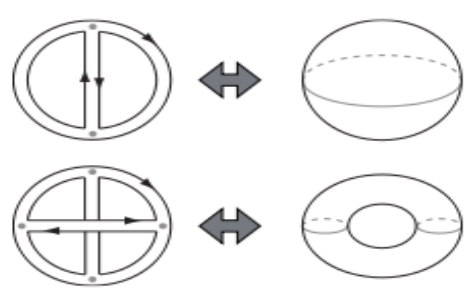

Figura 11: Analogía entre los diagramas de doble línea y su correspondiente diagrama topológico bidimensional.

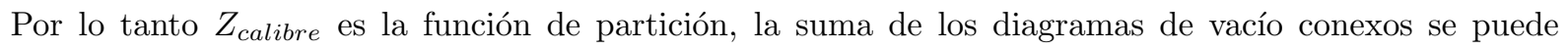
escribir:

$$
G_{\text {calibre }}=\ln Z_{\text {calibre }}=\sum_{g=0}^{\infty} N_{c}^{\chi} f_{g}(\lambda)
$$

Segunda clave de la correspondencia AdS/CFT: La función de partición de una teoría de gran $N_{c}$ está dada por una suma sobre las diferentes topologías de una superficie bidimensional.

Por otro lado, recordemos que hemos visto que cuando analizamos cuerdas en interacción (ver sección (2.4)), la función de partición $Z_{\text {string }}$ también resulta una suma sobre diferentes topologías bidimensionales de las amplitudes de las cuerdas cerradas. Ya que dentro del espectro de cuerdas cerradas se encuentra la gravedad, la expansión de cuerdas cerradas se corresponde a una expansión gravitacional perturbativa. En particular, la esfera se corresponde con la gravedad clásica (gráficos planares) mientras que el toro corresponde a la gravedad a 1-lazo (gráfico no planar).

De lo expuesto precedentemente podemos conjeturar un resultado extraordinario:

Una teoría de calibre puede ser representada por una teoría de cuerdas y en particular, en el límite de gran $N_{c}$, por una teoría de gravedad clásica.

En forma matemática esta conclusión puede ser expresada de acuerdo a (68) y (45) como:

$$
Z_{\text {calibre }}=Z_{\text {string }}
$$

Esta ultima ecuación nos vincula parámetros de ambas teorías:

$$
N_{c}^{2} \propto \frac{1}{g_{s}^{2}} \propto \frac{1}{G} \quad ; \quad \lambda \leftrightarrow l_{s}
$$

donde hemos usado (49). Pero además podemos aseverar:

- Una teoría de calibre en cuatro dimensiones en el límite de gran $N_{c}$ es representada por una ST. 
- Por otro lado una ST no es una teoría consistente en cuatro dimensiones.

De estas dos afirmaciones pareciese que hemos llegado a una contradicción, las dimensiones de las dos teorías no coinciden. Esta contradicción se resuelve con el siguiente razonamiento. La ST debiera admitir como solución muchos espacios, en particular un espacio plano y además espacios-tiempos curvados. Podremos usar espacios-tiempos curvos para representar teorías de calibre de gran $N_{c}$, con lo cual este espacio curvo puede tener una dimensionalidad mayor a cuatro. Por lo tanto la teoría de calibre puede ser representada por un espacio-tiempo curvo si tal espacio tiene simetría $I S O(1,3)$ (la invarianza de Poincaré, también llamado el grupo de Lorentz inhomogéneo) en cuatro de sus coordenadas. La aparición de un espacio-tiempo curvo de cinco dimensiones no es raro de esperar de acuerdo a la entropía de un BH. Un BH en cinco dimensiones tiene una entropía proporcional al área en cinco dimensiones (ver subsección (2.7.1)), pero un área en cinco dimensiones es un volumen en cuatro dimensiones, lo cual es lo correcto para la entropía de un sistema estadístico en cuatro dimensiones.

En conclusión diremos que:

Una teoría de calibre a gran $N_{c}$ es representada por un espacio-tiempo curvo de cinco dimensiones con una invarianza de Poincaré en cuatro dimensiones.

Una métrica en cinco dimensiones que tenga invarianza $I S O(1,3)$ es de la forma:

$$
d s_{5}^{2}=\Omega(w)^{2}\left(-d t^{2}+d \mathbf{x}_{3}^{2}\right)+d w^{2}
$$

donde $x^{\mu}=\left(t, \mathbf{x}_{3}\right)=(t, x, y, z)$. Esta métrica es manifiestamente invariante $I S O(1,3)$ en $\left(x^{\mu}\right)$, las coordenadas del espacio donde vive la teoría de calibre de gran $N_{c}$.

Ahora bien, todavía no sabemos nada acerca de que tipo de teoría de calibre es representada por un espacio-tiempo curvo. Tampoco sabemos mucho acerca de que tipo de espacio-tiempo curvo representa esta teoría de calibre (solo sabemos que tiene que tomar la forma de la métrica (71)) pues falta determinar el factor $\Omega(w)$. Impondremos una condición más para identificar la teoría de calibre y el espacio curvo correspondiente, la invarianza de escala que es una transformación del tipo:

$$
x^{\mu} \rightarrow a x^{\mu}
$$

En este tipo de teorías no existe una escala. Un ejemplo de teoría invariante de escala es la teoría de Maxwell, con los campos de calibre transformando como $A_{\mu} \rightarrow \frac{1}{a} A_{\mu}$. Más generalmente, si un campo transforma como

$$
\Phi \rightarrow a^{-\Delta} \Phi
$$

bajo la transformación (72) decimos que el campo tiene dimensión de escala $\Delta^{3}$. Pero es bien sabido que una teoría invariante de escala clásicamente en general no lo es cuánticamente. Por ejemplo la función beta a un lazo para una teoría de YM $S U\left(N_{c}\right)$ está dada por:

$$
\beta\left(g_{Y M}\right) \equiv \mu \frac{d g_{Y M}}{d \mu}=-\frac{11}{48 \pi^{2}} g_{Y M}^{3} N_{c}
$$

donde $\mu$ es la escala de energía y vemos que la función $\beta$ es distinta de cero y es negativa indicando que la teoría es asintóticamente libre.

No obstante existen teorías de calibre que son invariante de escala a nivel cuántico (la función beta de esta teoría es cero), notoriamente la teoría de super YM (SYM) $\mathbf{N}=\mathbf{4}$, donde $\mathbf{N}=\mathbf{4}$ indica que la teoría tiene cuatro supersimetrías. Su contenido de campos es:

- campo de calibre $A_{\mu}$

- 6 campos escalares $\phi_{i}$

- 4 fermiones de Weyl $\lambda_{I}$

No nos extenderemos más allá ya que en ningún momento realizaremos cálculos con ella.

Pasemos ahora al lado gravitatorio donde sí se trabajará a lo largo de esta tesis. La exigencia de invarianza de escala restringe la forma de la métrica (71) fijando $\Omega(w)=e^{-2 w / L}$ (se introduce la escala de longitud $L$ ya que $w$ tiene unidades de longitud). Haciendo $r=L e^{-w / L}$ la métrica (71) adquiere la forma:

$$
d s_{5}^{2}=\left(\frac{r}{L}\right)^{2}\left(-d t^{2}+d \mathbf{x}_{3}^{2}\right)+L^{2} \frac{d r^{2}}{r^{2}}
$$

\footnotetext{
${ }^{3}$ La dimension de escala es también llamada peso conforme o dimension conforme
} 
Este espacio de cinco dimensiones es conocido como espacio-tiempo de Anti-de-Sitter (AdS), siendo $L$ el radio de $A d S$. Por lo tanto este es el espacio-tiempo dual a una teoría de calibre de gran $N_{c}$ invariante de escala. El espacio $A d S_{5}$ es solución de la ecuación de Einstein con constante cosmológica negativa $\Lambda \equiv-6 / L^{2}$ :

$$
S_{5}=\frac{1}{16 \pi G_{5}} \int d^{5} x \sqrt{-g}\left(R_{5}-2 \Lambda\right)
$$

Hasta ahora analizamos dos simetrías, la simetría de Poincaré $I S O(1,3)$ y la invarianza de escala. En verdad la teoría de $S Y M \quad \mathbf{N}=\mathbf{4}$ y el espacio-tiempo $A d S_{5}$ presentan una simetría más grande que las incluye, la simetría conforme $S O(2,4)$.

\subsubsection{Espacio AdS}

Queremos ver en qué sentido el borde de un espacio $A d S_{d+1}$ es un espacio-tiempo plano en $d$ dimensiones. Tomamos $d=4$, el caso de interés.

Un espacio $A d S_{5}$ puede verse como un hiperboloide 5-dimensional en un espacio Lorentziano 6 dimensional. Probemos esto. Consideremos el espacio pseudo-minkowskiano en 6 dimensiones con métrica:

$$
d s^{2}=-d Y_{-1}^{2}-d Y_{0}^{2}+\sum_{i=1}^{4} d Y_{i}^{2}
$$

Definimos el hiperboloide de 5 dimensiones por el vínculo:

$$
-Y_{-1}^{2}-Y_{0}^{2}+Y_{1}^{2}+Y_{2}^{2}+Y_{3}^{2}+Y_{4}^{2}=-L^{2}
$$

Con el siguiente cambio de variables $U=Y_{-1}+Y_{4}, V=Y_{-1}-Y_{4}, X^{\alpha}=\frac{L}{U} Y^{\alpha} \operatorname{con} \alpha=0, \ldots, 3$, la métrica (77) toma la forma:

$$
d s^{2}=-d U d V+\frac{U^{2}}{L^{2}} \eta_{\alpha \beta} d X^{\alpha} d X^{\beta}+\eta_{\alpha \beta} X^{\alpha} X^{\beta} \frac{d U^{2}}{L^{2}}+2 \eta_{\alpha \beta} X^{\alpha} X^{\beta} \frac{U d U}{L^{2}}
$$

donde $\eta_{\alpha \beta}=(-,+,+,+)$. En las coordenadas $\left(U, V, X_{\alpha}\right)$ la hipersuperficie (78) se escribe:

$$
V=\eta_{\alpha \beta} X^{\alpha} X^{\beta} \frac{U}{L^{2}}+\frac{L^{2}}{U}
$$

Usando ésta en (79) y haciendo el cambio adicional $Z=\frac{L^{2}}{U}$ se obtiene la métrica inducida sobre el hiperboloide:

$$
d s^{2}=\frac{L^{2}}{Z^{2}}\left(d Z^{2}+\eta_{\alpha \beta} d X^{\alpha} d X^{\beta}\right)
$$

La ecuación (81) es la métrica de un espacio $A d S_{5}$ en coordenadas de Poincarè. El espacio al que tiende $A d S_{5}$ cuando $Z \rightarrow 0$ lo interpretaremos como el borde de $A d S_{5}$, la parte de la métrica de relevancia es el segundo término, o sea en la hipersuperficie $Z=0$ tenemos:

$$
d s_{A d S_{5}}^{2} \mid Z \rightarrow 0 \propto d s_{M i n k_{4}}^{2}
$$

Por lo tanto se puede decir que el borde del espacio $A d S_{5}$ es el espacio de Minkowski 4-dimensional a menos de un factor conforme global (divergente).

\subsubsection{Prescripción GKPW}

Escribiremos la ecuación (69) en la forma:

$$
Z_{C F T, \mathrm{~N}=4}=Z_{\text {String, }, A d S_{5}}
$$

En el lado izquierdo de la ecuación (83) figura la función de partición de una teoría de calibre con simetría conforme, mientras que en el lado derecho la función de partición de una teoría de cuerdas sobre $A d S_{5}$. Tal relación es conocida como la prescripción GKPW [5] [6].

Volviendo a la ecuación que vincula los parámetros de la teoría gravitacional y los de la teoría de calibre, ecuación (70), ya que el espacio $A d S$ nos introdujo la escala $L$ y dado que $\left[G_{5}\right]=L^{3}$, tenemos:

$$
N_{c}^{2} \sim \frac{L^{3}}{G_{5}}
$$


Por otro lado la constante de 't Hooft está relacionada a $l_{s}$ o lo que es lo mismo $\alpha^{\prime}$, y por lo tanto está relacionada a las correcciones en $\alpha^{\prime}$. En estas correcciones aparecen términos del tipo $l_{s}^{2} R$. Como la curvatura del espacio $A d S_{5}$ es $R \simeq 1 / L^{2}$ entonces:

$$
\lambda \sim\left(\frac{L}{l_{s}}\right)^{\star}
$$

donde $\star$ es algún coeficiente. Los coeficientes delante de (84) y en (85) pueden ser fijados usando D - branas, obteniéndose:

$$
N_{c}^{2}=\frac{\pi}{2} \frac{L^{3}}{G_{5}} \quad ; \quad \lambda=\left(\frac{L}{l_{s}}\right)^{4}
$$

Volviendo a (83), para evaluar el lado derecho no es necesario evaluar la función de partición completa, ya que en el límite de gran $N_{c}$ es suficiente usar la teoría de la gravitación clásica (pues $G_{5} \rightarrow 0$ ). Teniendo en cuenta esto, es posible usar la aproximación de punto silla, con lo cual:

$$
Z_{C F T}\left(N_{c}>>1\right)=e^{-\left.S_{E}\right|_{o n-s h e l l}+O\left(l_{s}^{2}\right)}
$$

donde $\left.S_{E}\right|_{\text {on-shell }}$ es la acción de Einstein euclídea evaluada sobre las soluciones clásicas de la métrica (en este caso el espacio-tiempo $\left.A d S_{5}\right)$.

El término $O\left(l_{s}^{2}\right)$ representa las correcciones en $\alpha^{\prime}$ (que representan correcciones cuánticas a la gravedad clásica de Einstein). Tales correcciones pueden ser ignoradas cuando la curvatura del espacio-tiempo es pequeña e ignorar las correcciones en $\alpha^{\prime}$ corresponde a tomar el límite $\lambda>>1$ del lado de la teoría de calibre. Por lo tanto llegamos a la relación final:

$$
Z_{C F T}\left(N_{c}>>1, \lambda>>1\right)=e^{-S_{E} \mid o n-s h e l l}
$$

Calcular $Z_{C F T}\left(N_{c}>>1, \lambda>>1\right)$ en este límite es difícil. No obstante el lado derecho de esta ecuación nos dice que puede ser evaluada usando supergravedad. Utilizaremos ecuación (88) como piedra angular a lo largo de la Tesis.

Notemos finalmente que el espacio-tiempo $A d S_{5}$ no es la única solución posible de (76). Un BH puede existir en $A d S_{5}$ y vimos que tiene temperatura (sección (2.7.2)) con lo cual podemos conjeturar que:

- Teoría de calibre a temperatura nula $\leftrightarrow$ espacio-tiempo $A d S_{5}$

- Teoría de calibre a temperatura finita $\leftrightarrow \mathrm{BH}$ en $A d S_{5}$

\subsubsection{D3-branas y teorías de calibre}

Describiremos ahora como las D-branas describen teorías de calibre. Para esto analizaremos las oscilaciones de cuerdas abiertas que viven sujetas a $N_{c}$ D3-branas.

Sabemos que la SST requiere de 10 dimensiones para que la teoría sea consistente o no tenga anomalías. Con lo cual las D3-branas se extienden en 3+1 dimensiones dentro de un espacio-tiempo de $9+1$ dimensiones. Cuando cuantizamos la teoría de Maxwell en D dimensiones el número de grados de libertad a especificar para los campos de calibre son $D-2=10-2=8$ en este marco, tenemos 8 en lugar de 2 grados de libertad, entonces, ¿cuáles son estos grados de libertad? ¿qué tipo de teoría de calibre se tiene en las D3branas? Para llevar a cabo el análisis recordemos de la sección (2.3) que del espectro de cuerdas abiertas sabemos que las oscilaciones sobre la brana representan campos de calibre mientras que las oscilaciones perpendiculares a la brana representan campos escalares no masivos, ambos en la representación adjunta de $U\left(N_{c}\right)$. Ya que las dimensiones espaciales del espacio-tiempo y de la brana son 9 y 3 respectivamente, el número de dimensiones perpendiculares a la brana es 6 y sabemos que hay un campo escalar no masivo por cada dimensión transversal a la brana. ${ }^{4}$ Los campos de calibre junto con los modos escalares llevan índices en la adjunta de $U\left(N_{c}\right)$. Ellos son parte (junto con los fermiones) de un supermultiplete vectorial de una teoría de SYM con supersimetría $\mathbf{N}=4$. Por ahora tenemos una teoría de SYM con $\mathbf{N}=4$ sobre la brana pero esta no es toda la historia. Además:

- El espectro de cuerdas abiertas contiene partículas masivas (los modos de oscilación más altos) que se acoplan a los estados de la brana.

- El espectro de cuerdas cerradas, SST contiene gravitones y mucho más (estados no masivos y masivos).

\footnotetext{
${ }^{4}$ Por supuesto que existen compañeros fermiónicos requeridos por la supersimetría pero no son relevantes para nuestro razonamiento.
} 
Con lo cual, desde este punto de vista no está claro si la gravedad puede ser ignorada. Primero consideremos la gravedad de la D-brana. Este es un objeto con energía que tiene asociado un tensor de energía-impulso que curva el espacio-tiempo. El potencial gravitacional debido a una partícula puntual es $\phi_{\text {Newton }} \approx G M / r$ en 4 dimensiones. En el caso a mano tenemos un objeto extendido con una densidad de masa $T_{3}$ e inmerso en un espacio-tiempo de 10 dimensiones con 6 dimensiones transversales, con lo cual:

$$
\phi_{\text {Newton }} \approx G_{10} T_{3} / r^{6-2}
$$

Dado que en ST la densidad de masa de $N_{c}$ D-branas es $T_{3} \sim \frac{N_{c}}{g_{s} l_{s}^{4}}{ }^{5}$, usando (49) el potencial(89) resulta:

$$
\phi_{\text {Newton }} \approx g_{s} N_{c} l_{s}^{4} / r^{4}=\lambda l_{s}^{4} / r^{4}
$$

Por otro lado la constante efectiva de la teoría de calibre es como vimos la constante de 't Hooft $\lambda$, y para cuerdas abiertas $g_{Y M}^{2} \approx g_{s}$ (ver ecuación (44)), de donde concluimos que:

- La constante de acoplamiento efectiva de la teoría de calibre es $\lambda=g_{Y M}^{2} N_{c}=g_{s} N_{c}$.

- La fuerza del acoplamiento de la brana a la gravedad es: $G_{10} T_{3} \sim g_{s} N_{c} l_{s}^{4}=\lambda l_{s}^{4}$.

Es posible mostrar que el sector gravitatorio (es decir los modos de cuerda cerrada sin masa, gravitones) dan lugar a un supermultiplete de gravedad en 10 dimensiones, cuya descripción efectiva esta dada por la acción de supergravedad de tipo IIB . Por lo tanto en el límite de bajas energías $E<<1 / l_{s}$ o lo que es lo mismo $l_{s} \rightarrow 0$ (manteniendo $E$ fijo) la teoría que los describe es una teoría de supergravedad de tipo IIB. Por otro lado vimos que los modos de cuerdas abiertas sin masa dan lugar a un supermultiplete vectorial $\mathbf{N}=4$ en $3+1$ dimensiones cuya descripción efectiva de baja energía esta dado por el Lagrangiano de una teoría SYM $U\left(N_{c}\right)$ con $\mathbf{N}=4$. En el caso de la brana, en el límite de $l_{s} \rightarrow 0$ y $\lambda \sim g_{s} N_{c}<<1$ tenemos gravedad debido a éstas despreciable pero seguimos manteniendo una teoría de calibre con una dinámica no trivial. Solo falta analizar los modos masivos, pero dado que sus masas $M \sim \frac{1}{l_{s}}$, los mismos se desacoplan a bajas energías, y resultan así irrelevantes. En conclusión, tenemos que las $N_{c}$ D 3 -Branas en el límite de bajas energías $l_{s} \rightarrow 0$ y acoplamiento débil $\lambda<<1$ vienen descriptas por:

$N_{c}$ D3-branas: $U\left(N_{c}\right) S Y M N=4+$ supergravedad en 10 dimensiones en un espacio-tiempo plano.
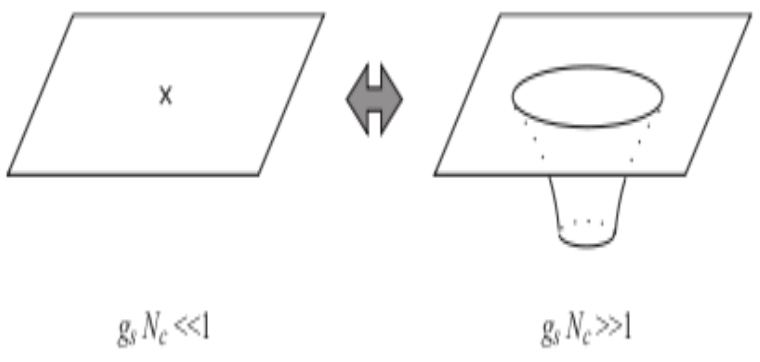

$g_{s} N_{c} \gg 1$

Figura 12: Izquierda: Cuando $g_{s} N_{c}<<1$ la curvatura del espacio-tiempo debido a la D3-Brana es pequeña y se puede aproximar la geometría por un espacio-tiempo plano. Derecha: Cuando $g_{s} N_{c}>>1$ una región con curvatura no despreciable del espacio-tiempo macroscópica aparece.

\subsubsection{D3-branas y espacio-tiempo curvo}

Hemos visto que $N_{c}$ D3-Branas en el límite de bajas energías y $\lambda<<1$ es descripta por una teoría effectiva de $\mathrm{SYM}$ con $\mathbf{N}=4$ supersimetrías inmersa en un espacio-tiempo de Minkowski de $9+1$ dimensiones. Esto no es cierto si $\lambda>>1$ ya que en tal caso la brana comenzara a curvar el espacio-tiempo de manera apreciable (ver ecuación (90)). Volviendo al argumento del potencial Newtoniano, en el límite de $r \rightarrow 0$ el espacio-tiempo aparece necesariamente curvado cerca del origen para valores:

$$
r^{4}<<g_{s} N_{c} l_{s}^{4}
$$

\footnotetext{
${ }^{5} \mathrm{La}$ dependencia en $1 / g_{s}=1 / g_{\text {open }}^{2}$ puede entenderse del hecho que una D-brana es interpretable como un solitón de Supergravedad.
} 
Si $g_{s} N_{c}<<1$, (91) nos dice que la región curvada es muy pequeña y puede pensarse que existe una fuente puntual representando la brana en un espacio-tiempo plano. Si en cambio $g_{s} N_{c}>>1$, el tamaño de $r$ en (91) no es pequeño y una zona macroscópica es curvada, ver figura (12). En el límite $g_{s} N_{c}>>1$ una descripción de Supergravedad es adecuada para describir la brana [17]. La solución de la D3-Brana extrema de Supergravedad es:

$$
d s_{10}^{2}=Z^{-1 / 2}\left(-d t^{2}+d \mathbf{x}_{3}^{2}\right)+Z^{1 / 2}\left(d r^{2}+r^{2} d \Omega_{5}^{2}\right)
$$

donde $Z=1+(L / r)^{4}, L=g_{s} N_{c} l_{s}^{4}, \mathrm{y} \mathbf{x}_{3}=(x, y, z)$ representan las direcciones espaciales en donde se extiende la brana. Estamos interesados en como la brana curva el espacio-tiempo cerca del horizonte $r=0$, así consideremos el límite $r<<L$ :

$$
d s_{10}^{2} \rightarrow(r / L)^{2}\left(-d t^{2}+d \mathbf{x}_{3}^{2}\right)+(L / r)^{2} d r^{2}+L^{2} d \Omega_{5}^{2}
$$

Por lo tanto la geometría de la D3-brana en el límite de horizonte cercano resulta ser $A d S_{5} \times S^{5}$. Así para $g_{s} N_{c}>>1$ tenemos que:

D3-brana: Supergravedad sobre $A d S_{5} \times S^{5}+$ Supergravedad en espacio-tiempo plano en 10 dimensiones.

En conclusión vimos que la descripción en términos de una teoría de calibre falla cuando $g_{s} N_{c}>>1$, mientras que la descripción en términos de supergravedad no es valida cuando $g_{s} N_{c}<<1$. Esto ultimo se puede ver mas fácil calculando el invariante $R^{M N Q P} R_{M N Q P} \simeq \frac{1}{g_{s} N_{c} l_{s}^{4}}$ de la métrica (93) dando $R^{M N Q P} R_{M N Q P} \simeq \frac{1}{g_{s} N_{c} l_{s}^{4}}$ podemos ver que cuando $g_{s} N_{c}<<1$ el escalar diverge. Por lo tanto la métrica (93) es confiable solo para $g_{s} N_{c}>>1$

\subsubsection{Teorías de calibre y espacio-tiempo curvo}

En las dos secciones anteriores vimos dos descripciones posibles de $N_{c}$ D3-branas, una que da lugar a una teoría de SYM $U(N) \mathbf{N}=4$ y otra una teoría de supergravedad en $A d S_{5} \times S^{5}$ y un sistema en común que se presenta en ambos análisis, supergravedad 10 dimensional en un fondo plano (ver figura (2.7.8)). Ya que aparecen en ambas descripciones y "se cancelan", podemos concluir que:

La teoría de SYM N=4 en 4 dimensiones es equivalente a la teoría de Supergravedad tipo IIB en

$$
A d S_{5} \times S^{5}
$$

Resumiendo: obtuvimos dos descripciones diferentes a partir de un mismo sistema, $N_{c}$ D3-branas, pero la parte crucial reside en que estas dos descripciones corresponden a diferentes límites (ver figura (2.7.8)). La primera es válida cuando $g_{s}<<1$ y $g_{s} N_{c}<<1$ mientras que la segunda es válida para $g_{s}<<1$ y $g_{s} N_{c}>>1$. Ya que en principio ambas teorías existen para todo $g_{s}$ y $g_{s} N_{c}$ es natural extrapolar la equivalencia para todo $g_{s}$ y $g_{s} N_{c}$, lo que constituye la versión fuerte de la conjetura de Maldacena [4].

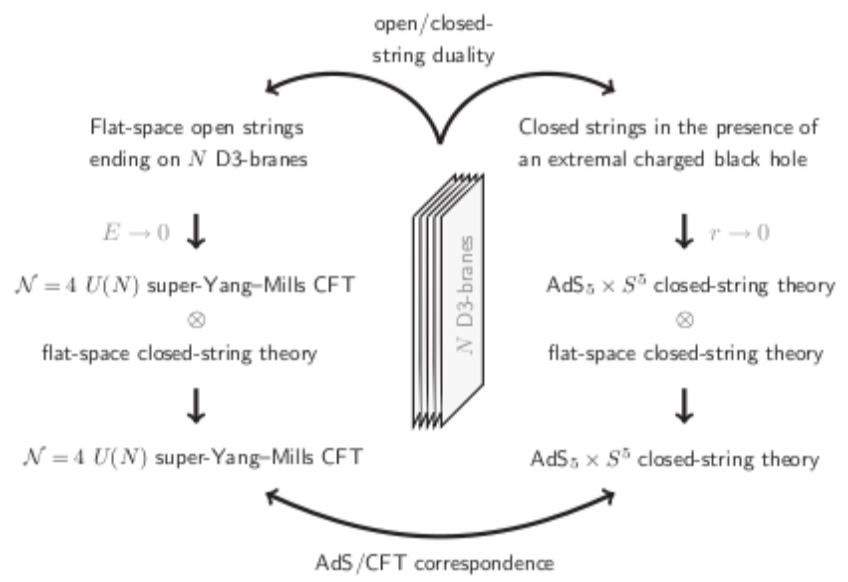

Figura 13: Se representa el esquema desde donde el cual se origina la correspondencia $A d S / C F T$. 


\subsubsection{Cálculo de funciones de correlación usando $A d S / C F T$}

Hemos visto gracias a la prescripción de GKWP que la función de partición de una ST es iguala la de una CFT. Esta igualdad es manejable en el límite de supergravedad (cuando $g_{s} \rightarrow 0 ; \frac{l_{s}}{L} \rightarrow 0$ ). Tal límite resulta ser la aproximación de punto silla donde se aproxima la función de partición por la exponencial on-shell de la acción de supergravedad (ver sección (2.7.5)). La igualdad entre las funciones de partición fue originalmente propuesta en espacio euclídeo, donde la métrica en $A d S_{d+1}$ toma la forma:

$$
d s_{E}^{2}=\frac{1}{z^{2}}\left(d z^{2}+d \tau^{2}+d \vec{x}^{2}\right)
$$

El objetivo inicial es calcular funciones de correlación de operadores $\hat{O}$ de la $C F T$. Para esto es conveniente introducir una fuente $J$ para el operador $\hat{O}$ :

$$
S_{C F T} \rightarrow S_{C F T}+\int d^{d} x J(x) \hat{O}(x)
$$

Por ejemplo, si consideramos el operador tensor energía-impulso: $\int d^{d} x h_{\mu \nu} \hat{T}^{\mu \nu}$. En el régimen de validez de (88) un campo $\psi$ viene dado por la solución clásica $\psi_{c l}$ de sus ecuaciones de movimiento. Cerca del borde $z=0$ de $A d S$ se comporta generalmente como:

$$
\psi_{c l}(x, z) \sim \psi_{-}(x) z^{\Delta_{-}}+\cdots+\psi_{+} z^{\Delta_{+}}+\ldots \quad, \quad z \rightarrow 0
$$

donde los exponentes $\Delta_{+}>\Delta_{-}$dependen de la masa y los números cuánticos de $\psi$ (por ahora no nos interesara su expresión exacta). El exponente $\Delta_{+} \equiv \Delta$ se corresponde con la dimensión conforme del operador $\hat{O}$ de la $C F T$. El primero y el segundo término se corresponden con modos no-normalizables y normalizables respectivamente, en el sentido de que la acción on-shell resulta en general divergente debido al termino $\psi_{-} z^{\Delta_{-}}$. El término de primer orden en (96) es identificado con la fuente del operador de campo dual $\hat{O}$ a través de:

$$
J(x) \equiv \lim _{z \rightarrow 0} z^{-\Delta_{-}} \psi_{c l}(x, z)=\psi_{-}(x)
$$

Pasemos ahora a igualar las funciones de partición

$$
Z_{C F T}=\left\langle e^{-\int d^{d} x J(x) \hat{O}(x)}\right\rangle_{C F T}=Z_{\text {Sugra }}\left[z^{\Delta_{-}} \psi_{c l}(x, z)_{z \rightarrow 0}=J(x)\right]
$$

Del lado izquierdo de (98) tenemos la $Z_{C F T}$ de la $C F T$ donde la fuente $J(x)$ es arbitraria. Del lado derecho tenemos la función de partición de supergravedad que en la aproximación saddle-point resulta ser como vimos anteriormente:

$$
Z_{\text {sugra }}[\psi] \simeq e^{-\left.S\left[\psi_{c l}\right]\right|_{\text {on-shell }}}
$$

Debido al comportamiento cerca del borde de $\psi_{c l}$ dado en (96) la acción on-shell diverge y por lo tanto es necesario regularizarla; la acción en (99) se entiende como la acción regularizada. Tales divergencias pueden ser regularizadas añadiendo contra-términos locales de borde determinados por el comportamiento de los campos cerca del borde. Tal procedimiento es llamado renormalización holográfica [16].

De (99) la generatriz de amplitudes conexas resulta:

$$
W_{C F T} \equiv \ln Z_{C F T}=-S_{r e n}\left[\left.\psi_{-}\right|_{\text {on-shell }}\right]
$$

Partiendo de (100) y derivando funcionalmente respecto a la fuente $\psi_{-}(x)$ se puede calcular la función de correlación de n-puntos:

$$
\left\langle\hat{O}\left(x_{1}\right) \hat{O}\left(x_{2}\right) \ldots \hat{O}\left(x_{n}\right)\right\rangle=-\left.\frac{\left.\delta^{n} S_{r e n}\left[\psi_{-}\right]\right|_{\text {on-shell }}}{\delta \psi_{-}\left(x_{1}\right) \delta \psi_{-}\left(x_{2}\right) \ldots \delta \psi_{-}\left(x_{n}\right)}\right|_{\psi_{-}=0}
$$

Para computar explícitamente la función de uno y dos puntos, es conveniente introducir el momento canónico conjugado renormalizado de la solución clásica $\psi_{c l}$ :

$$
\Pi_{r e n} \equiv \frac{\delta S_{r e n}\left[\psi_{c l}\right]}{\delta \psi_{c l}}
$$

Luego la función de 1 punto (valor de expectación de $\hat{O}$ ) a fuente finita $\psi_{-}$viene dada por:

$$
\langle\hat{O}(x)\rangle_{c}=-\left.\frac{\delta S_{r e n}\left[\psi_{-}\right]}{\delta \psi_{-}(x)}\right|_{\psi_{-}=0}=-\lim _{z \rightarrow 0} z^{-\Delta_{-}} \Pi_{r e n}(z, x)
$$


Por otro lado, se sabe de la teoría de respuesta lineal de la QFT que la respuesta de un sistema frente a una fuente externa $\psi_{-}$, es decir, como cambia $\langle\hat{O}(x)\rangle$ es:

$$
S \rightarrow S+\delta S=S+\int d^{4} x \psi_{-}(x) \hat{O}(x) \quad \Rightarrow \quad\langle\hat{O}(x)\rangle \rightarrow\langle\hat{O}(x)\rangle+\delta\langle\hat{O}(x)\rangle_{\psi_{-}}
$$

donde $\delta\langle\hat{O}(x)\rangle$ en espacio de Fourier resulta:

$$
\delta\left\langle\hat{O}\left(\omega_{E}, \vec{k}\right)\right\rangle_{\psi_{-}}=G_{R}\left(\omega_{E}, \vec{k}\right) \psi_{-}\left(\omega_{E}, \vec{k}\right)
$$

donde $G_{R}\left(\omega_{E}, \vec{k}\right)$ es la función de dos puntos (función de Green retardada) y $\omega_{E}$ es la frecuencia euclídea. Usando ecuaciones (103) y (105) obtenemos:

$$
G_{R}\left(\omega_{E}, \vec{k}\right)=\frac{\delta\left\langle\hat{O}\left(\omega_{E}, \vec{k}\right)\right\rangle_{\psi_{-}}}{\psi_{-}\left(\omega_{E}, \vec{k}\right)}=-\lim _{z \rightarrow 0} z^{-2 \Delta_{-}} \frac{\Pi_{r e n}}{\psi_{c l}}
$$

En el caso de un campo escalar la función de un punto resulta ser proporcional al modo normalizable en la expansión ultravioleta (UV) de $(96),\langle\hat{O}(x)\rangle_{\psi_{-}} \propto \psi_{+}(x)$, y entonces la función de correlación de dos puntos (funcion de Green) en el espacio de momentos resulta ser el cociente entre el modo normalizable y el no-normalizable:

$$
G_{R}\left(\omega_{E}, \vec{k}\right)=\frac{\psi_{+}\left(\omega_{E}, \vec{k}\right)}{\psi_{-}\left(\omega_{E}, \vec{k}\right)}
$$




\section{Materia Condensada}

\subsection{Diagrama de fases de los cupratos HTSC}

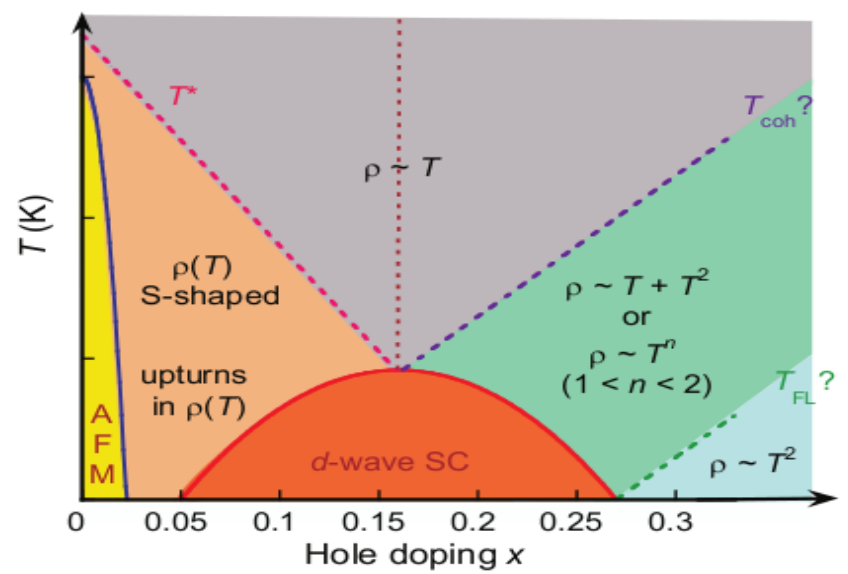

Figura 14: Se muestra el diagrama de fases de un cuprato dopado en términos de la temperatura y el dopaje. En cada fase se muestra el comportamiento de la resistividad con la temperatura. Las líneas sólidas dividen la fase normal de la fase superconductora y la fase antiferromagnética de la fase de pseudo-gap. Las líneas punteadas indican un cruce entre los diferentes comportamientos de la resistividad.

La superconductividad fue descubierta en 1911 por Heike Kamerlingh Onnes, quien observó que la resistencia eléctrica del mercurio desaparecía bruscamente al enfriarse a $4 K$. En 1986, se encontraron superconductores con una temperatura crítica más elevada, $T_{c} \approx 30 \mathrm{~K}$, por lo que se los conocen como superconductores de alta temperatura (HTSC). Son típicamente materiales de óxidos de cobre, comúnmente denominados cupratos. Por ejemplo el $\mathrm{La}_{2-x} S r_{x} C u \mathrm{O}_{4}$, donde $x$ indica el dopaje (de $\mathrm{La}$ y $\mathrm{Sr}$ en este caso). Del punto de vista de la estructura cristalina $\mathrm{Cu}-\mathrm{O}$ forman planos bidimensionales cuyos electrones sobre estos planos son los responsables de la superconductividad. Por ello se los puede pensar como un sistema en $2+1$ dimensiones. Todos los cupratos tienen un diagrama de fase universal, que se muestra en la figura (14). Se muestran diferentes fases de acuerdo a los distintos valores de temperatura y dopaje. Para valores bajos de dopaje el sistema se encuentra en una fase de tipo antiferromagnética. Conforme se aumenta el dopaje el sistema rápidamente pasa a la fase superconductora, en la forma de un domo, con un valor óptimo de $x$ para obtener el máximo valor de $T_{c}$. Si se sigue aumentando el dopaje, la temperatura crítica decrece y la fase superconductora se pierde dando lugar a un líquido de Fermi (zona azul). También vemos que existe una fase denominada de pseudo-gap donde el material presenta propiedades inusuales que todavía no son muy bien entendidas. Por encima del domo superconductor, el sistema se encuentra en una nueva fase denominada de metal extraño tampoco bien entendida, donde por ejemplo la resistividad escalea en forma lineal con la temperatura (a diferencia de un líquido de Fermi donde escalea en forma cuadrática). Entre la zona gris y la azul, se ve una región en la cual la resistividad va variando su comportamiento con $T$ de acuerdo al valor de dopaje presente (zona verde).

\subsection{Transiciones de fase: modelo de Ginzburg-Landau}

Cuando se cambia un parámetro de control como puede ser la temperatura, el sistema puede experimentar una cambio macroscópico tendiendo a un estado más estable que el anterior. Esto es lo que se conoce como una transición de fase. En tales procesos, los potenciales termodinámicos (como por ejemplo la energía libre) experimentan comportamientos no analíticos cerca de la temperatura crítica $T_{c}$. Se define una transición de fase de orden $n$ cuando la analiticidad de la energía libre es rota en la nth derivada. A saber:

- Transición de Fase de primer orden: $F$ es continua, pero $\frac{\partial F}{\partial T}$ no lo es.

- Transición de Fase de segundo orden: $F$ y $\frac{\partial F}{\partial T}$ son continuas pero $\frac{\partial^{2} F}{\partial T^{2}}$ no lo es.

La teoría de Ginzburg-Landau (GL) trata de explicar las transiciones de fase de segundo orden (aunque puede ser extendida para analizar transiciones de primer orden). Tales transiciones son caracterizadas por 
un parámetro denominado parámetro de orden, el cual resulta nulo por encima de cierta temperatura critica $T_{c}$ y distinto de cero debajo de $T_{c}$. Denotaremos a este parámetro como $\eta$. La idea consiste en proponer alguna forma para la energía libre del sistema en función de este parámetro de orden, ya que con la energía libre es posible calcular todas las propiedades termodinámicas del sistema. Para este fin consideramos lo siguiente:

- Como el parámetro de orden $\eta$ es pequeño cerca de la $T_{c}$ podemos hacer una expansión de Taylor alrededor de $\eta$

- Supondremos que la energía libre $F(T, \eta)$ como función de $\eta$ es par.

Así para $\eta$ chico tenemos:

$$
F(\eta, T)=F_{0}(T)+a(T)|\eta|^{2}+\frac{1}{2} b(T)|\eta|^{4}+\ldots
$$

donde $a(T)$ y $b(T)$ son parámetros fenomenológicos de la teoría. Suponemos que $b(T)>0$, ya que de no ser así $F(\eta, T)$ no tiene un mínimo estable. Si $a(T)>0$ entonces el valor mínimo de $\eta$ que minimiza $F(\eta, T)$ es cero. Si $a(T)<0$ entonces la condición de mínimo es: $|\eta|_{\min }^{2}=-a(T) / b(T)$. Considerando la temperatura $T_{c}$ para la cual $a\left(T_{c}\right)=0$ (o sea, $a(T)$ pasa de un valor positivo a otro negativo) tenemos entonces:

$$
|\eta|_{\text {min }}^{2}=\left\{\begin{array}{lll}
0 & , & T>T_{c} \\
-a(T) / b(T) \quad, & T<T_{c}
\end{array}\right.
$$

Por otro lado, cerca de $T_{c}$ podemos considerar las siguientes expansiones: $a(T)=a_{0}\left(T-T_{c}\right)+\ldots ., \mathrm{y}$ $b(T)=b_{0}+\ldots$, con $a_{0}, b_{0}>0$, lo que da lugar a:

$$
|\eta|_{\text {min }}=\left\{\begin{array}{lll}
0 & , & T>T_{c} \\
A\left(T_{c}-T\right)^{\frac{1}{2}} & , & T<T_{c}
\end{array}\right.
$$

mientras que con (110) en (108) obtenemos:

$$
F=F_{0}(T)+ \begin{cases}0 & , \quad T>T_{c} \\ -B\left(T_{c}-T\right)^{2} & ,\end{cases}
$$

donde $A \equiv \sqrt{\frac{a_{0}}{b_{0}}}$ y $B \equiv \frac{a_{0}^{2}}{2 b_{0}}$ Podemos ver que la entropía $S=-\frac{\partial F}{\partial T}$ es una función continua en $T$ mientras que el calor específico: $C_{V}=-T^{2} \frac{\partial^{2} F}{\partial T}$ no lo es, presentando un salto $\Delta C_{V}=2 B T_{c}{ }^{2}$ que indica que el sistema experimenta una transición de fase de segundo orden a $T=T_{c}$. Notemos además que para temperaturas menores a la crítica, la energía libre resulta menor que la de la fase normal, $F_{0}(T)$, como debe ser para la estabilidad del sistema.

\subsection{Modelo de Ginzburg-Landau para la Superconductividad}

En la mayoría de los metales la resistividad a bajas temperaturas se comporta de la forma:

$$
\rho=\rho_{0}+a T^{2}
$$

Pero por ejemplo para el platino, oro, etc. esto no ocurre y se presenta un comportamiento del tipo que se muestra en la figura (15).

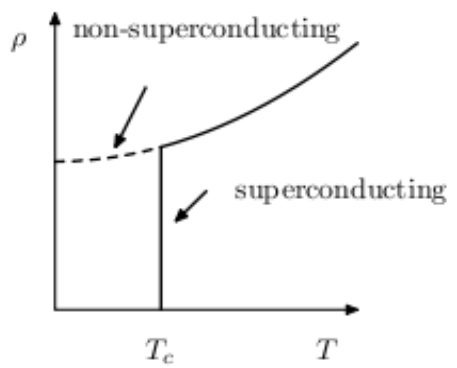

Figura 15: Comportamiento de la resistividad en función de la temperatura para los superconductores. 
A los materiales donde la resistividad resulta ser cero a partir de cierta temperatura critica $T_{c}$ se los denomina superconductores. Cabe destacar que, como veremos en la sección 3.4, un conductor perfecto no es un superconductor por lo que es necesario una teoría distinta para describir a este último.

Consideraremos la superconductividad/superfluidez como un fenómeno asociado a una transición de fase. Fenomenológicamente la superconductividad es una extensión del modelo más simple de GL:

$$
F\left[\psi ; T, A_{i}\right]=\frac{\hbar^{2}}{2 m_{*}}\left|D_{i} \psi\right|^{2}+a|\psi|^{2}+\frac{b}{2}|\psi|^{4}+\frac{1}{4} F_{i j}^{2}
$$

donde $D_{i} \equiv \partial_{i}-i \frac{e_{*}}{\hbar} A_{i}{ }^{6}$. Mencionaremos algunas características de este modelo

- El parámetro de orden $\psi$ en este caso es complejo, necesario para poder describir carga en el superconductor. Efectivamente, $\psi$ representa el valor de expectación de un par de Cooper, el cual consiste de dos electrones, por lo que $m_{*}=2 m$ y $e_{*}=2 e$ (ver sección (3.5))

- El sistema está acoplado a un campo electro-magnético, con lo cual el campo escalar al ser complejo estará cargado frente al campo de calibre $A_{i}$. Cuando no hay un campo externo el sistema es apropiado para describir un sistema de partículas neutras, tales como un superfluido.

Minimizando la energía libre (113) (o sea, exigiendo que $\frac{\partial F}{\partial \psi}=0, \frac{\partial F}{\partial \psi^{*}}$ obtenemos la ecuación de movimiento para $\psi$ :

$$
-\frac{\hbar^{2}}{2 m_{*}}\left(\vec{\nabla}-\frac{i e^{*} \vec{A}}{\hbar}\right)^{2} \psi(\vec{x})+\left(a+b|\psi|^{2}\right) \psi(\vec{x})=0
$$

La supercorriente puede ser encontrada derivando $F$ respecto a $\vec{A}$ :

$$
\overrightarrow{J_{s}}=-\frac{\partial F}{\partial \vec{A}}=-i \frac{\hbar e_{*}}{2 m_{*}}\left(\psi^{*} \nabla \psi-\psi \nabla \psi^{*}\right)-\frac{e_{*}^{2}}{m_{*}} \vec{A}|\psi|^{2}
$$

Escribiendo: $\psi(\vec{x})=|\psi(\vec{x})| e^{i e_{*} \phi(\vec{x}) / \hbar}$, la supercorriente es:

$$
J_{i}=\frac{e_{*}^{2}}{m_{*}}|\psi|^{2}\left(\partial_{i} \phi-A_{i}\right)
$$

En el calibre donde el bosón de Nambu-Goldstone es eliminado, $\phi=0$, llegamos a:

$$
J_{i}=-\frac{e_{*}^{2}}{m_{*}}|\psi|^{2} A_{i}=-\frac{1}{\lambda^{2}} A_{i} \quad ; \quad \lambda \equiv \frac{m_{*}}{e_{*}^{2}|\psi|^{2}}
$$

que es conocida como ecuación de London. De ella se puede inferir:

- Efecto Meissner: Consideremos un $\vec{A}=\left(0, A_{y}(x), 0\right)$, luego $F_{x y}=\partial_{x} A_{y}=B_{z}(x)$. Combinando la ecuación de London y la ecuación de Maxwell $\partial_{j} F^{i j}=J^{i}$ se tiene que:

$$
\partial_{x} F^{x y}=J^{y} \rightarrow-\partial_{x}^{2} A_{y}=-\frac{1}{\lambda^{2}} A_{y} \rightarrow B_{z}(x)=B_{0} e^{-x / \lambda}
$$

lo que significa que el campo magnético decae exponencialmente dentro de un superconductor

- Divergencia en la conductividad DC: Si tomamos la derivada temporal de la ecuación de London (117) se tiene que:

$$
\partial_{t} J_{i}=-\frac{1}{\lambda^{2}} \partial_{t} A_{i}=\frac{1}{\lambda^{2}} E_{i}
$$

y pasando a espacio de Fourier obtenemos:

$$
\tilde{J}_{i}(\omega, \vec{k}=0)=\frac{i}{\omega} \frac{1}{\lambda^{2}} \tilde{E}_{i}(\omega, \vec{k}=0)=\sigma(\omega) E_{i}(\omega, \vec{k}=0)
$$

Vemos que $\operatorname{Im}[\sigma(\omega)]$ tiene un polo en $\omega$. Esto implica una divergencia en la conductividad DC como explicaremos en la sección siguiente (ver (122)).

\footnotetext{
${ }^{6}$ Trabajaremos en el calibre $A_{0}=0$, que deja transformaciones residuales independientes del tiempo.
} 


\subsection{Conductor normal, perfecto y superconductor}

Para entender las características que presenta la conductividad, primero debemos entender la conductividad en un modelo mucho mas simple, denominado modelo de Drude para estudiar la respuesta electromagnética que presenta el sistema frente a un campo eléctrico externo. Este modelo también es útil para distinguir entre un conductor normal, conductor perfecto y un superconductor. El modelo de Drude usa la mecánica clásica para el movimiento del electrón, con lo cual:

$$
m \frac{d\langle\vec{v}\rangle}{d t}=e \vec{E}-m \frac{\langle\vec{v}\rangle}{\tau}
$$

donde $\langle\vec{v}\rangle$ es la velocidad media del electrón y $\tau$ el tiempo de relajación debido a las colisiones del electrón dentro del material.

- Conductor normal: La solución estacionaria de (121) viene dada por $\langle\vec{v}\rangle=\frac{e}{m} \vec{E} \tau$, la corriente es:

$$
\vec{J} \equiv n e\langle\vec{v}\rangle=\sigma \vec{E} \quad ; \quad \sigma=\frac{n e^{2} \tau}{m} E
$$

donde $n$ es la densidad de electrones de conducción. Ecuación (122) es la ley de Ohm donde $\sigma$ es la conductividad. Por lo tanto un conductor normal tiene una conductividad constante en el modelo de Drude.

- Conductor perfecto: Un conductor perfecto resulta ser el límite $\tau \rightarrow \infty$ (ya que en estas circunstancias no hay dispersión). Con lo cual la solución para campo constante resulta ser: $\langle\vec{v}\rangle=\frac{e}{m} \vec{E} t, \mathrm{y}$ la corriente obedece:

$$
\frac{\partial \vec{J}}{\partial t}=\frac{n e^{2}}{m} \vec{E}
$$

Vemos que es posible tener una corriente aún en ausencia de campo eléctrico.

Para calcular la conductividad a $\tau$ finito consideremos cómo responde el sistema a un campo eléctrico armónico de frecuencia angular $\omega, \vec{E}(t)=\vec{E}_{0} e^{-i \omega t}$. La corriente resulta: $\vec{J}(t)=\sigma \vec{E}(t)=\sigma E_{0} e^{-i \omega t}$. La solución es: $\langle\vec{v}\rangle=\vec{v}_{0} e^{-i \omega t}$ donde $\vec{v}_{0}=e / m \tau /(1-i \omega \tau) \vec{E}_{0}$ y por lo tanto la corriente viene dada por:

$$
\vec{J}(t)=\frac{n e^{2} \tau}{m} \frac{1}{1-i \omega \tau} \vec{E}(t) \quad \longrightarrow \quad \sigma(\omega)=\frac{\sigma}{1-i \omega \tau}
$$

Vemos que la conductividad es compleja, y en el límite de conductor perfecto $\tau \rightarrow \infty$ es:

$$
\operatorname{Re}[\sigma(\omega)]=\frac{n e^{2} \tau}{m} \frac{1}{1+\omega^{2} \tau^{2}} \longrightarrow \frac{n e^{2}}{m} \delta(\omega) \quad ; \quad \operatorname{Im}[\sigma(\omega)]=\frac{n e^{2} \tau}{m} \frac{\omega \tau}{1+\omega^{2} \tau^{2}} \longrightarrow \frac{n e^{2}}{m} \frac{1}{\omega}
$$

La parte real de la conductividad presenta una divergencia en $\omega=0$, mientras que la parte imaginaria presenta un polo simple. Más generalmente, las partes real e imaginaria de $\sigma(\omega)$ obedecen la relación de Kramers-Kronig:

$$
\operatorname{Im}[\sigma(\omega)]=-\frac{1}{\pi} \mathbf{P} \int_{-\infty}^{\infty} \frac{\operatorname{Re}\left[\sigma\left(\omega^{\prime}\right)\right]}{\omega^{\prime}-\omega} d \omega^{\prime}
$$

donde $\mathbf{P}$ denota el valor principal. De (126) se ve que una función delta en $R e[\sigma(\omega)]$ se refleja en un polo $1 / \omega$ en $\operatorname{Im}[\sigma(\omega)]$.

De (120) y (125) vemos que la conductividad presenta las mismas características en los superconductores y en el modelo de Drude de conductor perfecto, por lo tanto la divergencia en la conductividad DC no es adecuada para distinguirlos. Para diferenciarlos reescribamos la ecuación (123):

$$
\partial_{t} J_{i}=-\frac{1}{\lambda_{D}^{2}} \partial_{t} A_{i} \quad ; \quad \lambda_{D}^{2}=\frac{m}{n e^{2}}
$$

Vemos que (127) es la derivada temporal de la ecuación de London, de donde se desprende que (117) implica (127), pero lo contrario no es cierto. La diferencia surge en la respuesta magnética del sistema. Tomando el rotor de la ecuación (117) se obtiene:

$$
\nabla \times \vec{J}+\frac{1}{\lambda^{2}} \vec{B}=0
$$


Si la corriente es cero el campo magnético dentro del superconductor resulta ser nulo. Por otro lado tomando el rotor de (127) (que es la derivada de la ecuación de London deducida del modelo de Drude) se obtiene:

$$
\frac{\partial}{\partial t}\left(\nabla \times \vec{J}+\frac{1}{\lambda_{D}^{2}} \vec{B}\right)=0
$$

que implica que si la corriente es cero el campo magnético es constante dentro del conductor perfecto, y por lo tanto no presenta necesariamente efecto Meissner.

\subsection{Superconductividad microscópica: ideas físicas}

En 1957 J. Bardeen, L. Cooper y J. Schrieffer (BCS) propusieron una teoría microscópica de la superconductividad. Sus principales logros son las predicciones de la formación de pares de Cooper y la existencia de un salto de energía $E_{g}=2 \Delta$ a la energía de Fermi $(\mathrm{FE})$.

Primeramente en la teoría de GL (secciónes (3.2) y (3.3)) para explicar la superconductividad, el conjunto de los electrones superconductores se describe, como vimos en la sección (3.3), por medio de la función de onda $\psi(\vec{r})$ con una sola coordenada espacial, a diferencia de la función de onda de un sistema de $n$ electrónes interactuantes que depende de $\vec{r}_{1}, \vec{r}_{2}, \ldots, \vec{r}_{n}$ es decir, con $\psi(\vec{r})$ se describe el comportamiento colectivo de todos los electrones superconductores.

Una de las principales dificultades en formular una teoría de superconductividad radicaba en el desconocimiento de la interacción que ocasionaba el comportamiento colectivo de los electrones. Sabemos que en un metal los electrones tienen energías de Fermi $E_{F}$ del orden de varios electrón-voltios y que el estado superconductor se destruye cuando $k_{B} T \approx 10^{-4} \mathrm{eV}$. Por lo tanto había que hallar una interacción muy débil con participación de la red ${ }^{7}$ capaz de ordenar a los electrones a pesar de la gran energía de estos.

La formación de pares de Cooper surge de la existencia de una fuerza atractiva entre electrones cercanos a la superficie de Fermi (FS), debida a la interacción con los fonones (excitaciones de la red de iones). Cooper descubrió que dos electrones fuera del mar de Fermi pueden atraerse a través del siguiente mecanismo. Para explicar el origen de la interacción atractiva entre los electrones se debe tener en cuenta el efecto del movimiento de los iones al pasar el electrón. La idea es que un electrón atrae los iones positivos y polariza el medio. Éstos a su vez atraen a un segundo electrón dando lugar a una interacción efectiva neta entre los dos electrones. BCS también se dieron cuenta que la FS resultaba inestable a la formación de pares de Cooper, debido a que la energía de un electrón dentro del par es menor que la FE.

El segundo mayor resultado fue la predicción de un gap de energía $E_{g}=2 \Delta$. Este gap es la energía necesaria para romper el par de Cooper. Si la frecuencia del campo eléctrico es mayor que $E_{g}(0)$, el par se disocia y la conductividad normal reaparece. A $T=0$ la energía del gap esta relacionada a la temperatura crítica vía:

$$
E_{g}(0)=2 \Delta(0)=2.52 k_{B} T_{c}
$$

donde $k_{B}$ es la constante de Boltzmann. Este valor se verifica para una gran rango de superconductores como se puede mostrar en la figura (16)

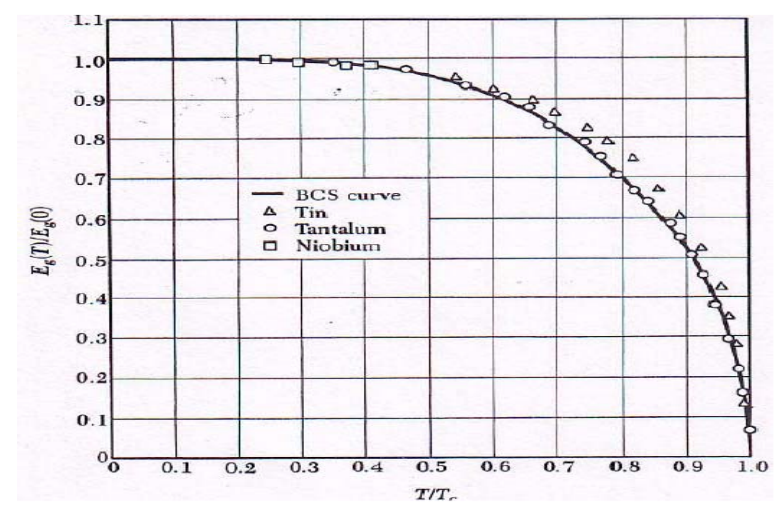

Figura 16: Se muestra la energía del gap para diferentes materiales como función de la temperatura.

\footnotetext{
${ }^{7}$ Efecto isotópico: Las muestras de semiconductores hechas de distintos isótopos de un mismo elemento poseen temperaturas críticas diferentes, $T_{c} \sim 1 / \sqrt{m_{\text {isotopo }}}$. Esto muestra que en la superconductividad la interacción de los electrones con las vibraciones de la red juega un rol crucial.
} 


\subsection{Gas de Fermi}

Un gas de Fermi consiste en $N$ electrones no interactuantes en una caja. Un estado del sistema puede escribirse como producto de estados individuales de cada uno de los electrones:

$$
\Psi=\psi_{1} \ldots \psi_{N} \quad ; \quad \psi_{i}=\psi_{i}(\vec{x}, t) \chi_{i s}
$$

donde $\psi_{i}(\vec{x}, t)$ es la parte orbital y $\chi_{i s}$ es la parte de espín del electrón i- ésimo. Las partículas van ocupando estados de acuerdo al principio de exclusión de Pauli. La energía de cada una es: $\epsilon_{0}(k)=\frac{\hbar^{2} k^{2}}{2 m_{e}}$. Se define la energía de Fermi $E_{F}$, como la energía del nivel más alto ocupado a $T=0 K$ (en este caso la FE coincide con el potencial químico $\mu$ ). El momento de Fermi está definido por:

$$
E_{F}=\frac{\hbar^{2} k_{F}^{2}}{2 m_{e}}
$$

Las excitaciones que se producen en un gas de Fermi corresponden a mover un electrón por encima del nivel de Fermi (partícula) o remover un electrón dentro de la FS (agujero):

$$
\epsilon(\vec{k})=\epsilon_{0}(k)-E_{F}
$$

El estado del sistema está determinado por la función de distribución $\eta_{\vec{k} \sigma}$. Para un gas de Fermi, la energía viene dada por:

$$
E\left[\eta_{\vec{k} \sigma}\right]=\sum_{\vec{k} \sigma} \epsilon_{0}(\vec{k}) \eta_{\vec{k} \sigma}
$$

En particular para el estado fundamental, la función de distribución es la de Fermi-Dirac: $\eta_{\vec{k} \sigma}^{0}=\theta\left(k_{F}-k\right)$; la energía del estado fundamental (GS) resulta:

$$
E_{G S} \equiv E\left[\eta_{\vec{k} \sigma}^{0}\right]=\sum_{\vec{k} \sigma} \epsilon_{0}(\vec{k}) \eta_{\vec{k} \sigma}^{0}
$$

Por lo tanto la energía de excitación del sistema es:

$$
\delta E\left[\eta_{\vec{k} \sigma}\right] \equiv E\left[\eta_{\vec{k} \sigma}\right]-E_{G S}=\sum_{\vec{k} \sigma} \epsilon(\vec{k}) \delta \eta_{\vec{k} \sigma} \quad ; \quad \delta \eta_{\vec{k} \sigma}=\eta_{\vec{k} \sigma}-\eta_{\vec{k} \sigma}^{0}
$$

Podemos argumentar que al entregar energía, por ejemplo calentándolo, la misma no es percibida por todos los electrones sino solo por aquellos que están más cerca de la FE. Esto es así puesto que los electrones que se encuentran en niveles muy inferiores a $E_{F}$, en virtud del principio de exclusión, no pueden participar del movimiento térmico debido a que no pueden pasar al siguiente nivel energético por estar ocupado pues sería necesaria mucha energía para que estos electrones puedan ser excitados a estados desocupados. Por lo tanto a bajas temperaturas los electrones cerca de la FS son los que definen las propiedades del sistema. Cerca de la FS podemos hacer un desarrollo de Taylor de $\epsilon(\vec{k})$ alrededor de $k_{F}$ :

$$
\epsilon(\vec{k})=\epsilon\left(k_{F}\right)-\mu+\left.\frac{d \epsilon}{d k}\right|_{k=k_{F}}\left(k-k_{F}\right)+O\left(\left(k-k_{F}\right)^{2}\right)=v_{F}\left(k-k_{F}\right)+O\left(\left(k-k_{F}\right)^{2}\right)
$$

donde $v_{F}=\left.\frac{d \epsilon}{d k}\right|_{k=k_{F}}$ es la velocidad de Fermi ${ }^{8}$.

\subsection{Líquido de Fermi y metales extraños}

El líquido de Fermi o Landau-Fermi es un modelo teórico que permite describir fermiones en interacción bajo ciertas condiciones físicas cuando la temperatura es suficientemente baja. Por ejemplo, el Helio-3 $\left(H e^{3}\right)$ es un líquido de Fermi a bajas temperaturas (pero no tan bajas como para que estar en su fase superfluida), como lo son los electrones en un metal normal (no superconductor).

En un líquido no existen estados cuánticos para las partículas o átomos individuales, solo podemos referirnos a los niveles que corresponden a estados estacionarios del sistema, de modo que para construir el espectro de un líquido de Fermi debemos partir de la hipótesis de adiabaticidad: cuando se encienden

\footnotetext{
${ }^{8}$ Más generalmente, la FS está definida por la ecuación: $\epsilon(\vec{k})=0$, o sea, por los momentos cuya energía de excitación es nula. En el caso de sistemas invariantes ante traslaciones, como el gas de Fermi en cuestión, resulta ser una esfera de radio $k_{F}$. La velocidad de Fermi por otro lado está definida por: $\left.\vec{v}_{F} \equiv \vec{\nabla}_{\vec{k}} \epsilon(\vec{k})\right|_{\epsilon(\vec{k})=0}$. En el caso del gas de Fermi en cuestión, $v_{F}=k_{F} / m$ y las correcciones en (137) son calculables. No obstante cuando existen interacciones la expansión (137) es igualmente relevante, ver ecuación (138).
} 
gradualmente las interacciones la clasificación de los niveles de energía permanece invariable cuando pasamos del gas al líquido, o sea, los números cuánticos de un sistema no interactuante siguen siendo buenos números cuánticos incluso después que la interacción se ha encendido. En esta correspondencia uno a uno entre los autoestados del sistema interactuante con los del gas de Fermi, el papel de partícula del gas lo juegan las excitaciones elementales llamadas cuasi-partículas.

En estos sistemas interactuantes la energía total ya no es más la suma de excitaciones individuales. En primer término, cuando la cuasi-partícula se mueve, la interacción con el mar de Fermi hace que su inercia aumente dando lugar a un cambio en la masa efectiva $m \rightarrow m_{*}$. En segundo término la energía total del sistema interactuante cambia debido a que la función de distribución $\eta_{\vec{k} \sigma}$ de las cuasi-partículas se ve afectada por la interacción entre éstas. La energía de excitación del sistema a segundo orden en $\delta \eta_{\vec{k} \sigma}$ esta dada por:

$$
\delta E \equiv \sum_{\vec{k}, \sigma} v_{F}\left(k-k_{F}\right) \delta \eta_{\vec{k} \sigma}+\frac{1}{2} \sum_{\vec{k} \vec{k}^{\prime} \sigma \sigma^{\prime}} f\left(\vec{k}, \vec{k}^{\prime}, \sigma, \sigma^{\prime}\right) \delta \eta_{\vec{k} \sigma} \delta \eta_{\vec{k}^{\prime} \sigma^{\prime}}+\ldots
$$

Las cuasi-partículas son solo aproximaciones de los autoestados del Hamiltoniano del sistema real. Se puede calcular la vida media de estos autoestados aproximados (que son las cuasi-partículas) considerando la tasa de decaimiento de una cuasi-partícula de energía $\epsilon$ por encima de la FS:

$$
\frac{1}{\tau} \sim \epsilon^{2}+T^{2}
$$

donde el segundo término tiene en cuenta el efecto de la temperatura. De (139) vemos que cerca de la superficie de Fermi $\epsilon \approx 0$ la cuasi-partícula es siempre bien definida en el sentido de que $\tau>>1 / \epsilon$ (podemos decir entonces que $\tau^{*}=1 / \epsilon$ es el tiempo mínimo requerido para que se desarrolle adiabáticamente el sistema y entonces se cree la cuasi-partícula). Para valores grandes de $\epsilon, \tau<<1 / \epsilon$ la cuasi-partícula decae antes de que la interacción pueda ser completamente encendida. Asi la descripción de Landau es válida siempre y cuando la energía de las excitaciones y la temperatura sean suficientemente bajas.

De acuerdo a lo visto precedentemente, la teoría de Landau se asienta sobre la hipótesis que las excitaciones creadas añadiendo una partícula al sistema no interactuante pueden ser descriptas por una partícula libre con una gran vida media, la cuasi-partícula. Justamente la respuesta causal de un sistema interactuante cuando se añade una partícula a éste es la función de Green retardada, cuya forma general es:

$$
G_{R}(\vec{k}, \omega)=\frac{1}{\omega-\epsilon(\vec{k})-\Sigma(\vec{k}, \omega)} \quad ; \quad \Sigma=\operatorname{Re} \Sigma+i \operatorname{Im} \Sigma
$$

La función espectral, que mide la variación de la densidad de estados por unidad de energía del sistema, se define como:

$$
A(\omega, \vec{k}) \equiv \frac{1}{\pi} \operatorname{Im}_{R}(\vec{k}, \omega)=\frac{1}{\pi} \frac{\operatorname{Im} \Sigma(\omega, \vec{k})}{(\omega-\epsilon(\vec{k})-\operatorname{Re} \Sigma(\omega, \vec{k})))^{2}+(\operatorname{Im} \Sigma(\omega, \vec{k}))^{2}}
$$

A bajas energías $\omega \approx 0$ y alrededor de $k_{F}$ podemos expandir $G_{R}^{-1}$ en potencias de $k-k_{F}$ y $\omega \approx 0$. Con las definiciones:

$$
\begin{gathered}
Z^{-1} \equiv 1-\left.\frac{\partial \operatorname{Re} \Sigma\left(k_{F}, \omega\right)}{\partial \omega}\right|_{\omega=0} \\
\left.\tilde{\epsilon}(\vec{k}) \equiv\left(k-k_{F}\right) Z \frac{\partial(\epsilon(\vec{k})+\operatorname{Re} \Sigma(\vec{k}, 0))}{\partial \vec{k}}\right|_{k=k_{F}}=\left(k-k_{F}\right) k_{F} / m^{*} \\
\frac{m}{m^{*}} \equiv Z\left(1+\left.\frac{m}{k_{F}} \frac{\partial \operatorname{Re} \Sigma(k, 0)}{\partial k}\right|_{k=k_{F}}\right) \\
\frac{1}{\tau_{\vec{k}}(\omega)} \equiv-2 Z \operatorname{Im} \Sigma(\vec{k}, \omega)
\end{gathered}
$$

se obtiene

$$
G_{R}(\vec{k}, \omega)=Z\left(\omega-\tilde{\epsilon}(\vec{k})+\frac{i}{2 \tau_{\vec{k}}(\omega)}\right)^{-1}
$$

de donde tomando la parte imaginaria se obtiene

$$
A(\omega, \vec{k})=\frac{Z / \tau_{\vec{k}}(\omega)}{(\omega-\tilde{\epsilon}(\vec{k}))^{2}+\left(\frac{1}{2 \tau_{\vec{k}}(\omega)}\right)^{2}}
$$


Vemos de (147) que la función espectral tiene la forma de una Lorentziana cerca de $k=k_{F}$. La contribución del pico de cuasi-partícula en la función espectral se puede extraer considerando valores pequeños de $\operatorname{Im} \Sigma$ y usando la fórmula $\delta(x)=\frac{1}{\pi} \lim _{a \rightarrow 0} \frac{a}{x^{2}+a^{2}}$ :

$$
A_{q p}(\vec{k}, \omega) \equiv 2 \pi Z \delta(\omega-\tilde{\epsilon}(\vec{k}))
$$

Esto muestra que la función espectral tiene un pico para $\omega=\tilde{\epsilon}(\vec{k})$ que es identificado con la cuasi-partícula (ver figura (17)). Si bien la regla de suma de estados:

$$
\int_{-\infty}^{\infty} \frac{d \omega}{2 \pi} A(\vec{k}, \omega)=1
$$

se debe satisfacer, no es verificada dado que $A_{q p}$ no es toda la función espectral. Considerando la integral del resto de la función espectral $A^{\prime}(\vec{k}, \omega)$ tendremos un valor de $1-Z$. Por lo tanto podemos escribir:

$$
A(\vec{k}, \omega)=2 \pi Z \delta(\omega-\tilde{\epsilon}(\vec{k}))+A^{\prime}(\vec{k}, \omega)
$$

La contribución de $A^{\prime}$ no esta asociada con el polo, sino que contiene una estructura de excitaciones más complicada que no son descriptas por un pico de cuasi-partícula.

(a)

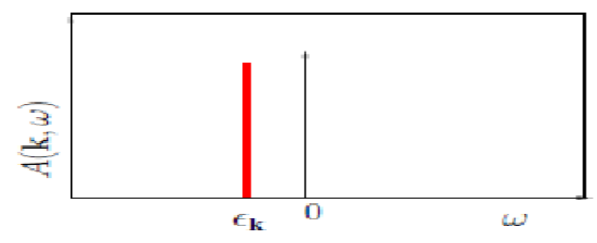

(b)

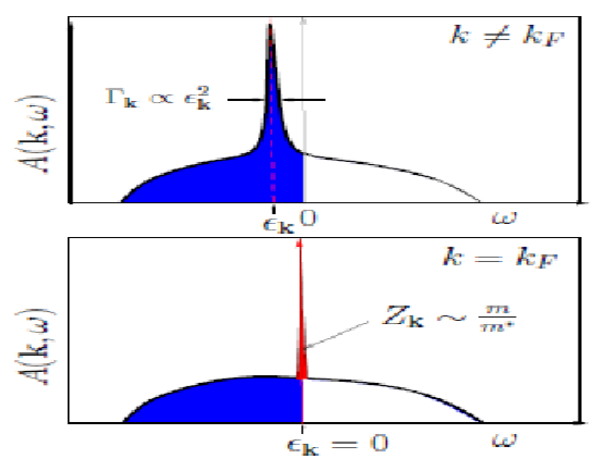

Figura 17: La figura (a) muestra la función espectral de un sistema de electrones no interactuantes a $\vec{k}$ fijo. Esta resulta ser una delta para $\omega=\epsilon_{k}$. La figura (b) muestra la función espectral para un líquido de Fermi. Esta contiene un pico característico para $k \neq k_{F}$ con un cierto ancho $\Gamma_{k}$ para $\omega=\epsilon_{k}$. Tal pico es identificado con el pico de una cuasi-partícula. Si $k=k_{F}$ este pico se vuelve infinitamente agudo correspondiente a una gran vida media de la cuasi-partícula. El peso en el pico de la cuasi-partícula es $Z_{k} \sim m / m^{*}$.

Pasemos ahora a hablar sobre algunas propiedades de los denominados metales extraños, que corresponden a una región del diagrama de fases de un HTSC cuyas propiedades no son compatibles con las predichas por la teoría de líquido de Fermi (ver figura (14)). Tal fase sí presenta una FS, pero la representación de cuasipartícula no es aplicable, ya que la vida media de tales excitaciones resultan mucho menores. Esto puede observarse experimentalmente en los experimentos de ARPES para los cupratos HTSC. Dichos resultados experimentales se pueden ajustar con la siguiente expresión para la función de Green:

$$
\begin{gathered}
G_{R}(\omega, \vec{k})=\frac{h}{\omega-v_{F}\left(k-k_{F}\right)+\Sigma(\omega, \vec{k})} \\
\Sigma(\omega, \vec{k}) \approx c \omega \ln \omega+d \omega \quad ; \quad c, h \varepsilon \mathbb{R} y d \varepsilon \mathbb{C}
\end{gathered}
$$

Ya que $d \varepsilon \mathbb{C}$ de $(151)$ se obtiene que $\Gamma \sim \operatorname{Im}(\Sigma) \propto \omega$. Asimismo en estos sistemas el comportamiento de la resistividad resulta ser lineal con la temperatura. En un líquido de Fermi-Landau ambas dependencias son cuadráticas. 


\section{Modelos holográficos de la Materia Condensada}

\subsection{Superconductividad holográfica}

En esta sección explicamos en detalle el artículo [7], de donde por otro lado son extraídas todas las figuras. Aunque ambos superconductores, los LTSC y HTSC pueden ser descriptos por pares de Cooper, el mecanismo de apareamiento es bastante diferente en el caso de los HTSC, y no pueden ser descriptos por la teoría BCS. El mecanismo usual de interacción fonón-electrón no está presente en los HTSC ya que ningún efecto isotópico se ha detectado. ${ }^{9}$

Esto parece indicar que hay un mecanismo de acoplamiento fuerte en lugar de uno débil en los HTSC y por lo tanto la correspondencia $A d S / C F T$ podría ser de ayuda en estos casos ya que se encuentran en el rango de validez donde es aplicable. Por lo tanto la pregunta que surge es si es posible modelar holográficamente este tipo de sistemas. Los ingredientes mínimos serían los siguientes:

- Buscamos describir una teoría de campos, con lo cual la teoría gravitatoria dual debe originar un tensor de energía-impulso conservado en la QFT.

- Debe existir la noción de temperatura del superconductor.

- Una característica única de un superconductor es la resistividad nula o la divergencia de la conductividad como vimos en la sección (3.3). Para ver esto necesitamos una corriente $U(1)$.

- Un estado superconductor es un fenómeno asociado con una transición de fase. Vimos en las secciones (3.2 y que 3.3) que necesitamos un condensado que modele los pares de Cooper.

De acuerdo al diccionario de $A d S / C F T$, si tenemos en el borde operadores del tipo $\hat{T}^{a b}, \hat{J}^{a}$ y $\hat{O}$ entonces esto se corresponde con una métrica en el bulk $g_{\mu \nu}$, un campo de Maxwell $A_{\mu}$ y un campo escalar complejo $\psi$ (que juega el papel del condensado) respectivamente. Por lo tanto terminamos con una teoría del tipo Einstein-Maxwell-campo escalar cargado. Además buscaremos soluciones de BH ya que éstos introducen la temperatura en la QFT como vimos en la sección 2.7.2.

Para describir al superconductor desde el lado gravitatorio es necesario que el agujero negro no tenga pelo a altas temperaturas, es decir, que el sistema solo esté especificado por los parámetros del BH, mientras que a bajas temperaturas presente pelo, es decir, que el sistema esté caracterizado por un valor no trivial del campo además de los parámetros del BH. En [19] se observó que agujeros negros acoplados a un campo escalar neutro $\phi$ con un potencial arbitrario $V(\phi)$ en un espacio $A d S$ puede solo formar pelo a bajas temperaturas si el espacio $A d S$ se vuelve inestable. En un trabajo subsiguiente [11] se mostró que un campo escalar cargado alrededor del $\mathrm{BH}$ presenta las propiedades deseadas. En la referencia [7] la acción que se propone para modelar los superconductores desde el lado gravitatorio es de la forma:

$$
S_{s u}=\int d^{4} x \sqrt{-g}\left(R+2 \Lambda-\frac{1}{4} F_{\mu \nu} F^{\mu \nu}-|\nabla \phi-i q A \phi|^{2}-m^{2}|\phi|^{2}\right)
$$

Variando la acción respecto a la métrica, al campo de Maxwell y al campo escalar obtenemos las tres ecuaciones diferenciales:

$$
\begin{gathered}
-\left(\nabla_{\mu}-i q A_{\mu}\right)\left(\nabla^{\mu}-i q A^{\mu}\right) \phi+\frac{1}{2} \frac{\phi}{|\phi|} V^{\prime}(|\phi|)=0 \\
\nabla^{\mu} F_{\mu \nu}=i q\left[\phi^{*}\left(\nabla_{\nu}-i q A_{\nu}\right) \phi-\phi\left(\nabla_{\nu}+i q A_{\nu}\right) \phi^{*}\right] \\
R_{\mu \nu}-\frac{g_{\mu \nu}}{2} R-\frac{3 g_{\mu \nu}}{L^{2}}=\frac{1}{2} F_{\mu \rho} F_{\nu}^{\rho}-\frac{g_{\mu \nu}}{8} F^{\rho \gamma} F_{\rho \gamma}-\frac{g_{\mu \nu}}{2} V(|\phi|) \\
-\frac{g_{\mu \nu}}{2}|\nabla \phi-i q A \phi|^{2}+\frac{1}{2}\left[\left(\nabla_{\mu} \phi-i q A_{\mu} \phi\right)\left(\nabla_{\nu} \phi^{*}+i q A_{\nu} \phi^{*}\right)+\mu \leftrightarrow \nu\right]
\end{gathered}
$$

con $\Lambda=-3 / L^{2}$ y $\phi$ el campo escalar cargado de masa $m$ y carga $q$. Para simplificar este sistema lo que se puede hacer es trabajar en el límite de prueba, donde los efectos del campo escalar y del campo de calibre sobre la métrica son despreciables. Tal límite se obtiene reescaleando los campos como: $\phi \rightarrow \phi / q \mathrm{y} A \rightarrow A / q$, y luego tomando $q \rightarrow \infty$, De este modo se mantiene el acoplamiento entre $\phi$ y $A_{\mu}$ mientras que sus ecuaciones

\footnotetext{
${ }^{9}$ Efecto isotópico: La frecuencia de Debye $\omega_{D}$ (frecuencia máxima a la que pueden vibrar los átomos que forman la red) verifica: $\omega_{D} \propto \frac{1}{\sqrt{m_{\text {iones }}}}$. Fue encontrado que la temperatura de transición a la fase superconductora para el mercurio muestra la misma dependencia pero sustituyendo ${ }^{220} \mathrm{Hg}$ con un isotopo diferente, el ${ }^{198} \mathrm{Hg}$, es decir, $T_{c} \propto \frac{1}{\sqrt{m_{i s o t o p o}}} \cdot$ Este efecto sugiere que la superconductividad está relacionada a las vibraciones de la red.
} 
se desacoplan de la gravedad, con lo cual podemos considerar estos campos en un fondo gravitacional fijo solución de (156). Se elegirá como tal fondo a un agujero negro de Anti-de-Sitter (AdSBH). Tomaremos además el potencial de la forma $V(\phi)=m^{2}|\phi|^{2}$, con un valor de la masa: $m^{2}=-2 / L^{2}$ lo que implica que en principio $\phi$ es taquíónico, pero como demostraron Breitenlohner y Freedman (BF) en un espacio $A d S$ existe un rango de masas negativas que no vuelven al sistema inestable:

$$
-\frac{d^{2}}{4}<L^{2} m^{2}<-\frac{d^{2}}{4}+1 \longrightarrow-\frac{9}{4}<L^{2} m^{2}<-\frac{5}{4}
$$

donde consideramos $d=3$, con lo cual $m^{2}=-2 / L^{2}$ satisface (157). La masa asociada al campo $\phi$ es el factor que multiplica a $|\phi|^{2}$ con lo cual si se desarrolla la expresión $|\nabla \phi-i q A \phi|^{2}$ se desprende un termino $q^{2}|A|^{2}$ que multiplica a $|\phi|^{2}$, obteniéndose en (163) una masa efectiva:

$$
m_{e f f}^{2}=m^{2}+g^{t t} q^{2} A_{t}^{2}
$$

De (158) puede apreciarse porqué en este sistema podríamos tener un agujero negro con pelo a bajas temperaturas, pues $m_{e f f}^{2}$ puede ser negativa ya que $g^{t t}$ en nuestras convenciones es negativa lo que daría lugar a desestabilizar el campo escalar. Por lo tanto al disminuir la temperatura el BH se vuelve más extremo implicando que $g_{t t}$ desarrolle un doble cero en el horizonte, o sea que $g^{t t}$ se hace cada vez más grande y el sistema más inestable.

Una de las razones principales de que tengamos pelo a baja temperatura es que el espacio es asintóticamente $A d S$ y no asintóticamente plano. Expliquemos brevemente porqué sucede esto. Sea $Q_{i}$ la carga inicial del $\mathrm{BH}$, si $q Q_{i}$ es suficientemente grande, el campo eléctrico cerca del horizonte será intenso, lo cual vía mecanismo de Schwinger se crearan pares de partículas cargadas (el campo eléctrico tiene suficiente fuerza como para sacar dos partículas del vacío y hacerlas reales). La partícula con carga opuesta al BH caerá a este, reduciendo la carga del $\mathrm{BH}$ mientras que la partícula con igual signo del $\mathrm{BH}$ será repelido por este. En un espacio asintóticamente plano estas partículas escaparan al infinito sin nada que las detenga y el estado final será un BH de Reissner-Nördstrom con $Q_{f}<Q_{i}$. Pero en un espacio asintóticamente $A d S$ tales partículas no podrán escapar debido a la presencia de la constante cosmológica negativa. Luego las partículas se dirijiran de nuevo al BH. Una vez allí habrá cierta probabilidad que se dispersen con alguna partícula que ya se encuentra en el horizonte. Este proceso llegará a un equilibrio, dando lugar a un gas con carga opuesta al BH por encima del horizonte. Esto resultará ser el superconductor que flota por encima del horizonte del BH debido a un equilibrio entre el campo gravitacional y el campo eléctrico. Este gas de partículas es la descripción del pelo y es la solución que hallaremos a continuación.

Buscamos soluciones estacionarias, con un horizonte de $\mathrm{BH}$ negro planar (el horizonte tiene una extensión infinita ) y con solo la componente eléctrica del campo de calibre encendida. En el límite de prueba estudiaremos $\phi$ y $A_{\mu}$ en el fondo de un BH de Anti-de-Sitter (AdSBH). El ansatz es:

$$
d s^{2}=-f(r) d t^{2}+\frac{d r^{2}}{f(r)}+r^{2}\left(d x^{2}+d y^{2}\right) \quad ; \quad A(r, t, x, y)=\phi(r) d t \quad ; \quad \psi(r, t, x, y)=\psi(r)
$$

La solución para $f(r)$ es:

$$
f(r)=\frac{r^{2}}{L^{2}}\left(1-\frac{r_{0}^{3}}{r^{3}}\right)
$$

La componente radial de la ecuación de Maxwell resulta:

$$
0=i\left(\psi^{*} \partial_{r} \psi-\psi \partial_{r} \psi^{*}\right)
$$

de donde se concluye que la fase de $\psi$ es constante y entonces se puede tomar a $\psi$ real. Por otro lado las ecuaciones de movimiento para el campo de calibre y el campo escalar son:

$$
\begin{gathered}
\phi^{\prime \prime}+\frac{2}{r} \phi^{\prime}-\frac{2 \psi^{2}}{f} \phi=0 \\
\psi^{\prime \prime}+\left(\frac{f^{\prime}}{f}+\frac{2}{r}\right) \psi^{\prime}+\left(\frac{\phi^{2}}{f^{2}}+\frac{2}{L^{2} f}\right) \psi=0
\end{gathered}
$$

que es un sistema de dos ecuaciones diferenciales acopladas de segundo orden. La solución de vacío, o sea, sin pelo, está dada por: $\psi=0, \phi=\mu-\frac{\rho}{r}$. Estamos interesados obviamente en soluciones con $\psi \neq 0$. Para encontrar dichas soluciones es necesario dar cuatro condiciones de contorno.

- Para que $A_{\mu}$ tenga norma finita en el horizonte es necesario que $\phi\left(r_{0}\right)=0$. Esta condición impone que en la solución trivial: $\rho=r_{0} \mu$. 
- Si evaluamos (163) en $r_{0}$ llegamos a que $\psi^{\prime}\left(r_{0}\right) \propto \psi\left(r_{0}\right)$, por lo cual solo necesitamos un parámetro para especificar $\psi$ en el horizonte.

- Hemos reducido el problema a especificar dos constantes $\psi\left(r_{0}\right)$ y $\phi^{\prime}\left(r_{0}\right)$.

Resulta de interés como vimos en la sección (2.7.9) calcular los comportamientos asintóticos. Las expansiones asintóticas de los campos $(r \rightarrow \infty)$ son:

$$
\begin{gathered}
\psi \sim \frac{A_{1}}{r}+\frac{A_{2}}{r^{2}}+\ldots=\frac{\psi^{(1)}}{r}+\frac{\psi^{(2)}}{r^{2}}+\ldots \\
\phi \sim B_{1}+\frac{B_{2}}{r}+\cdots=\mu-\frac{\rho}{r}+\ldots
\end{gathered}
$$

donde $\mu$ resulta ser el potencial químico de la teoría del borde y $\rho$ la densidad de carga. Notemos que el superconductor que se está modelando tiene $\rho \neq 0$ aunque los superconductores ordinarios son eléctricamente neutros. Esto puede ser explicado notando que el modelo de superconductor holográfico no está modelando electrones sino el condensado, o sea los pares de Cooper. Éstos son bosones (por eso la introducción del campo escalar), pero en ningún momento se incluyen cargas positivas proveniente de la red atómica (iones). Es más, la presencia de simetría traslacional en el modelo indica que no se está teniendo en cuenta dicha red sino solo los pares de Cooper.

Surge ahora la pregunta: ¿cómo se comporta el condensado como función de la temperatura?. Para esto necesitamos analizar como varían las soluciones $\psi^{1}$ y $\psi^{2}$ como función de $T$. Usando simetrías de escala podemos fijar: $L=1$ y $r_{0}=1$. Con esto la temperatura del $\mathrm{BH}$ pasa a ser:

$$
T=\frac{3 r_{0}}{4 \pi L^{2}} \rightarrow T=\frac{3}{4 \pi}
$$

lo que da lugar a una temperatura constante. En principio podríamos pensar que hemos cometido un error al usar las simetrías de escala ya que esto ha conducido a una temperatura constante que no podemos variar con ningún parámetro. Esto no es correcto ya que las cantidades invariantes de escala son las relevantes. Hagamos ahora algo de análisis dimensional, $\rho$ tiene unidades de $q / V$ haciendo $q=1$ y como nuestro sistema es $2 \mathrm{~d},[\rho]=1 /[L]^{2}$. Si $c=\hbar=1$ entonces $[M]=[L]^{-1}$ con lo cual $[\rho]=[M]^{2}$. Por otro lado se ve de (166) que $[T]=[M]$. Por lo tanto para construirnos una cantidad adimensional con la temperatura, se tomaran cualquiera de las siguientes cantidades:

$$
\frac{T}{\sqrt{\rho}}=\frac{T}{\mu}=\frac{3}{4 \pi \sqrt{\rho}}=\frac{3}{4 \pi \mu}
$$

Por lo tanto para cada valor de $\rho$ o $\mu$ indistintamente, los valores $\psi^{(1)}$ o $\psi^{(2)}$ varían con lo cual varían con la temperatura. Para especificar la solución debemos dar un valor de $\mu$ y podemos hacer $\psi^{(1)}=0$ o $\psi^{(2)}=0$ ya que ambos modos son normalizables en este caso ${ }^{10}$. O sea, tenemos dos modelos posibles, de acuerdo a la prescripción GWKP:

$$
\psi^{(1)}=0 \quad, \quad\left\langle\hat{O}_{2}\right\rangle=\psi^{(2)} \quad o \quad \psi^{(2)}=0 \quad, \quad\left\langle\hat{O}_{1}\right\rangle=\psi^{(1)}
$$

Como $\psi^{(i)}$ son interpretados como fuentes, poner la fuente a cero y tener un valor de expectación no nulo indica una rotura espontánea de simetría.

Siendo $[\psi]=0 \rightarrow\left[\psi^{(2)}\right]=[M]^{2}$; de manera análoga $\left[\psi^{(1)}\right]=[M]$ con lo cual las cantidades adimensionales que podemos construir con los $\langle\hat{O}\rangle$ son $\frac{\sqrt{\left\langle\hat{O}_{2}\right\rangle}}{T_{c}}$ y $\frac{\left\langle\hat{O}_{1}\right\rangle}{T_{c}}$. Las soluciones numéricas graficando estos condensados como función de $T / T_{c}$, se aprecian en la figura (18).

\footnotetext{
${ }^{10}$ Vale la pena acotar que númericamente estamos resolviendo el sistema de ecuaciones diferenciales mediante el método de disparo, es decir damos valores a $\psi\left(r_{0}\right)$ y $\phi^{\prime}\left(r_{0}\right)$ para que $\mu$ y $\psi^{(1)}\left(\mathrm{o} \psi^{(2)}\right)$ tengan el valor deseado.
} 

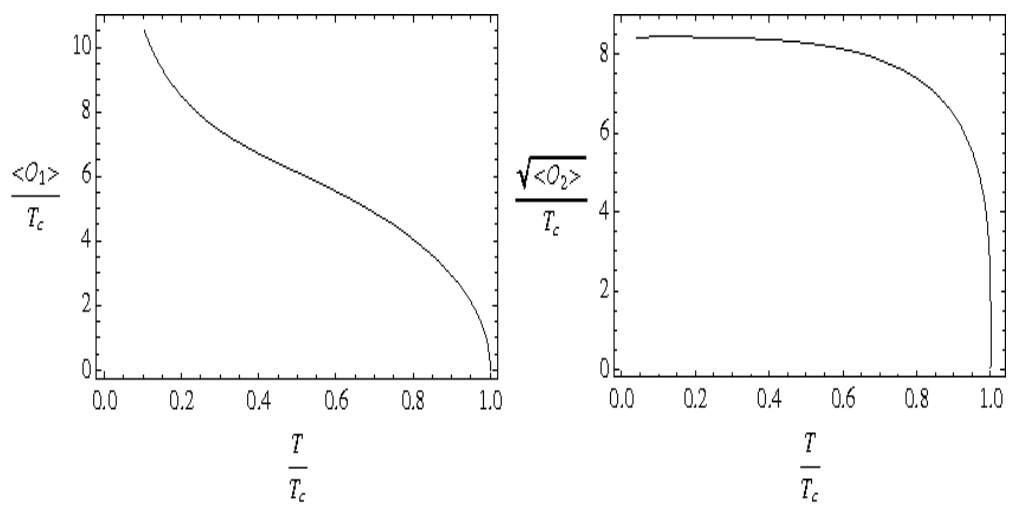

Figura 18: Izquierda: Se muestra el condensado $\left\langle\hat{O}_{1}\right\rangle$ en función de $\frac{T}{T_{c}}$. Se puede ver rápidamente que para temperaturas muy bajas el valor del condensado diverge. Derecha: Se muestra el condensado $\left\langle\hat{O}_{2}\right\rangle$ en función de $\frac{T}{T_{c}}$. Éste representa un valor finito de $\left\langle\hat{O}_{2}\right\rangle$ para temperaturas bajas, dando lugar a un valor del condensado finito.

De la figura (18) de la derecha se ve un comportamiento muy similar al obtenido en la teoría BCS en la figura (16)), donde la identificación de la energía del gap con la raiz del condensado se deduce de la figura (21). Es decir que cuando la temperatura tiende a cero, el valor del condensado tiende a un valor constante. En el condensado de la izquierda se ve notoriamente que tal valor diverge a bajas temperaturas. Esto puede ser explicado con el siguiente argumento: cuando disminuimos la temperatura disminuye la masa del BH haciéndose comparable a la masa del campo escalar y entonces ambos objetos curvan el espacio con la misma intensidad y no es posible despreciar el efecto de $\psi$ ni $A_{\mu}$ sobre la métrica. Si ajustamos las curvas para valores pequeños del condensado (cerca de $T_{c}$ ), se encuentra un comportamiento de raíz cuadrada, lo cual es típico de una transición de fase de segundo orden (ver sección (3.2)). Específicamente se encuentra:

$$
\left\langle\hat{O}_{1}\right\rangle \approx 9.3 T_{c}\left(1-T / T_{c}\right)^{1 / 2} \quad ; \quad\left\langle\hat{O}_{2}\right\rangle \approx 144 T_{c}^{2}\left(1-T / T_{c}\right)^{1 / 2}
$$

También es posible calcular conductividades (que son funciones de Green, o sea, funciones de correlación de dos puntos como se mostró en la sección (2.7.9)). La conductividad $\sigma_{i j}(\omega)$ caracteriza la respuesta del sistema a un campo eléctrico (luz de frecuencia $\omega$ ) a lo largo de la direcciôn normal a la coordenada radial $r$ es decir, en el espacio $(t, x, y)$ donde vive la muestra. Con lo cual, para calcular $\sigma_{i j}(\omega)$ se envían fotones desde el borde $A d S_{4}$ y se observa como ellos son absorbidos o reflejados por el condensado en el horizonte. Pasemos a ver como podemos hacer esto.

Perturbaremos el campo de Maxwell $A_{t}$ mediante fluctuaciones vectoriales de momento nulo y con una dependencia temporal de la forma $e^{-i \omega t}$. La parte del orden siguiente al primero de esta perturbación se corresponderá de acuerdo a la prescripción GKPW con el valor esperado de la corriente en el sistema y ésta a su vez, vía la ley de Ohm se relaciona con la conductividad. Consideremos una perturbación a la solución (159) de la forma:

$$
\delta g_{x t}(r, t, x, y)=\epsilon g_{x t}(r) e^{-i \omega t} \quad ; \quad \delta A(r, t, x, y)=\epsilon A_{x}(r) e^{-i \omega t}
$$

donde $\epsilon$ es un parámetro que controla la misma. Reemplazando en las ecuaciones de movimiento y quedándonos a orden líneal se arriba a un sistema de ecuaciones diferenciales para $g_{x t}$ y $A_{x}(r)$ que puede desacoplarse, obteniéndose para $A_{x}(r)$ la ecuación:

$$
A_{x}^{\prime \prime}+\frac{f^{\prime}}{f} A_{x}^{\prime}+\left(\frac{\omega}{f^{2}}-\frac{2 \psi^{2}}{f}\right) A_{x}=0
$$

El comportamiento de la solución de esta ecuación diferencial cuando nos acercamos al horizonte $r_{0}$ (donde $f\left(r_{0}\right)=0$ ) es de la forma: $A_{x} \sim A_{1} f(r)^{ \pm \frac{i \omega}{3 r_{0}}}$. Para calcular la respuesta causal del sistema, es decir, la función de Green retardada, se imponen las condiciones de borde entrantes en el horizonte [79]. Por lo tanto la solución relevante es: $A_{x} \sim A_{1} f(r)^{-\frac{i \omega}{3 r_{0}}}$. Luego el comportamiento cerca del borde resulta:

$$
A_{x}=A_{x}^{(0)}+\cdots+\frac{A_{x}^{(1)}}{r}+\ldots
$$

El diccionario holográfico nos dice que la fuente y el valor expectación de la corriente son:

$$
A_{x}=A_{x}^{(0)} \quad ; \quad\left\langle J_{x}\right\rangle=A_{x}^{(1)}
$$


De acuerdo a la ley de Ohm la conductividad es:

$$
\sigma(\omega)=\frac{\left\langle J_{x}\right\rangle}{E_{x}}=-\frac{\left\langle J_{x}\right\rangle}{\dot{A_{x}}}=-\frac{i\left\langle J_{x}\right\rangle}{\omega A_{x}}=-\frac{i A_{x}^{(1)}}{\omega A_{x}^{(0)}}
$$
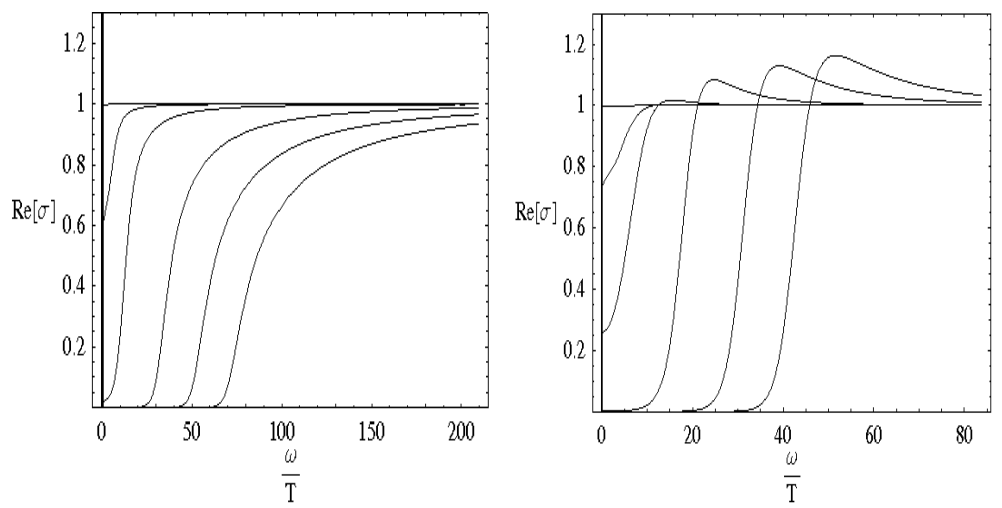

Figura 19: Izquierda: Se muestra la parte real de la conductividad para los condensados $\left\langle\hat{O}_{1}\right\rangle$ (izquierda) y $\left\langle\hat{O}_{2}\right\rangle$ (derecha) en función de la frecuencia para distintas temperaturas, con $\frac{T}{T_{c}}$ decreciente de izquierda a derecha en cada gráfico. Una función delta en $\omega=0$ está también presente.

En la figura (19) se muestran los gráficos de la parte real de la conductividad en función de la frecuencia para diferentes temperaturas. La línea horizontal corresponde a la conductividad por encima de la temperatura crítica mientras que las otras curvas corresponden a temperaturas más bajas que $T_{c}$. A medida que se reduce la temperatura aparece un salto de energía similar al encontrado usando la teoría BCS. Por debajo de esta energía la conductividad desaparece Esto es análogo a lo que sucede en el límite de superconductividad $\tau \rightarrow 0$ (ver sección (3.4)).

En la figura (20) se muestran los gráficos de la parte imaginaria de la conductividad. Una divergencia en $\omega=0$ está presente, de acuerdo con la relación de Kramers-Kronig (126) ya que se debe a la presencia de una delta de Dirac $\delta(\omega)$ en la figura (19), aunque no se la puede apreciar numéricamente. Esto es exactamente lo obtenido en el límite de conductor perfecto en el modelo de Drude en sección (3.4).
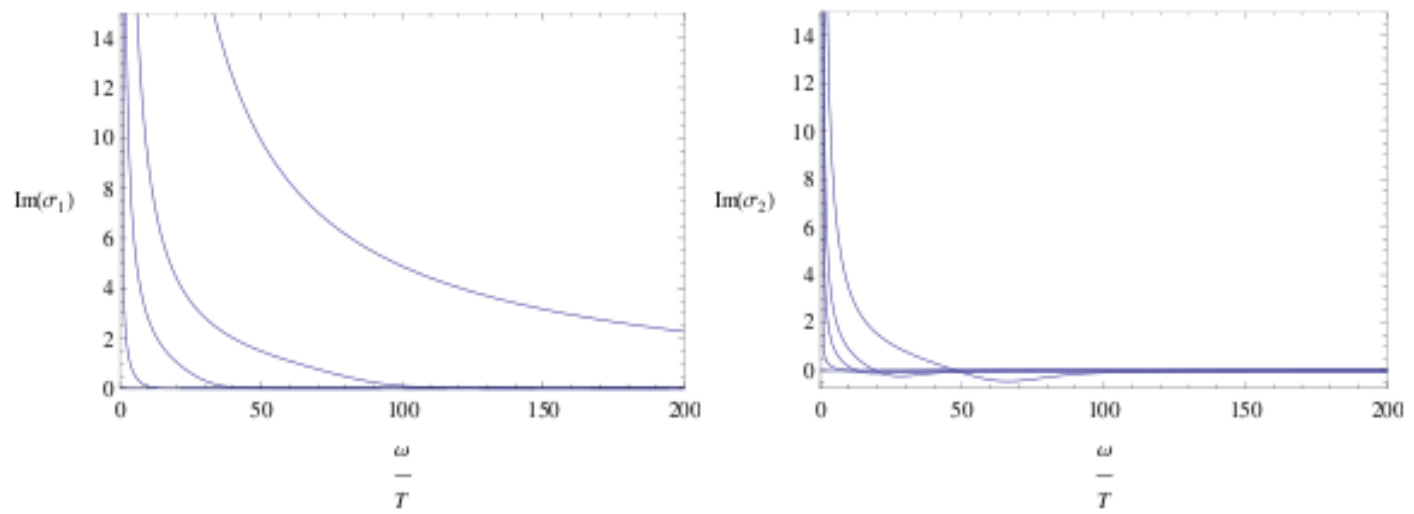

Figura 20: Se muestra la parte imaginaria de la conductividad para temperaturas $T<T_{c}$ para ambos condensados.

Para saber más acerca de la magnitud del salto de energía se pueden analizar soluciones a temperaturas cercanas a la crítica. En las figuras (21) se muestra la parte real de la conductividad en función de la frecuencia normalizada en términos del condensado. 

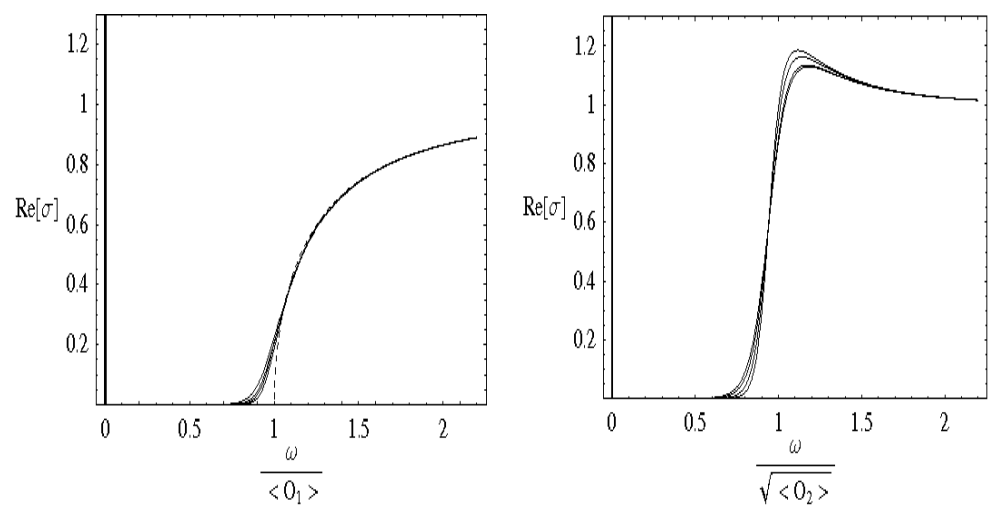

Figura 21: Se muestra la parte real de la conductividad en función de la frecuencia normalizada con el condensado correspondiente, para valores pequeños de temperatura.

En ellas se observa para todas las temperaturas $T<<T_{c}$ un salto de energía finito aproximadamente en el valor del condensado, $\omega_{g} \approx \sqrt{\left\langle\hat{O}_{i}\right\rangle}$. De la figura (18) de la derecha el valor del condensado a $T \sim 0$ tiende aproximadamente a $8.4 T_{c}$. Podemos decir entonces que a bajas temperaturas el salto de energía para el segundo condensado es:

$$
\omega_{g}=2 \Delta \approx 8.4 T_{c}
$$

Este valor difiere apreciablemente del valor que predice la teoría BCS, $2 \Delta \approx 3.52 T_{c}$. Esto no es de extrañar ya que las condiciones de validez de la correspondencia $A d S / C F T$ se verifican a acoplamiento fuerte. Ya que el acoplamiento en los pares de Cooper es mucho más intenso, $\omega_{g}$ (que es la energía de disociación del par) es mucho más alta. Se ha mostrado que para superconductores holográficos en $2+1$ y $3+1$ dimensiones, la relación $(175), \frac{\omega_{g}}{T_{c}} \approx 8.4$, se verifica con un error en las computaciones menor al 10 por ciento. Por otra parte este valor coincide experimentalmente con el cociente medido en HTSC [30].

Podemos hacer un rápido cálculo analítico para explicar el valor constante de la conductividad para $T>T_{c}$. La solución del sistema para esta región de temperaturas es la trivial:

$$
d s^{2}=\frac{L^{2}}{z^{2}}\left(-d t^{2}+d \vec{x}^{2}\right) \quad ; \quad \psi=0 \quad ; \quad \phi=\mu-\frac{\rho}{r}
$$

donde $z \equiv L^{2} / r$. La solución de (171) es: $A_{x}=e^{-i \omega(t-z)}$ (donde se ha anulado la onda saliente del horizonte del $\mathrm{BH})$, y puede ser expandida de la siguiente forma cerca del borde:

$$
A_{x}=e^{-i \omega t}(1+i \omega z+\ldots)=e^{-i \omega t}\left(1+\frac{i \omega L^{2}}{r}+\ldots\right)
$$

Comparando (177) con (172) y de acuerdo a (174):

$$
\sigma_{i j}(\omega)=\frac{i}{\omega} G_{i j}^{R}(\omega, k=0) \quad ; \quad G_{x x}^{R}=-\frac{2}{\kappa} \frac{A_{x}^{(1)}}{A_{x}^{(0)}}
$$

de donde:

$$
\sigma_{x x}=\sigma_{y y}=\frac{2 L^{2}}{\kappa^{2}} \quad ; \quad \sigma_{x y}=0
$$

ya que el parámetro de orden, siendo un escalar rompe la invarianza de calibre pero no la invarianza rotacional.

Vale la pena destacar lo siguiente. Los fenómenos de interacción fuerte en una QFT pueden ser entendidos desde la teoría dual usando una representación geométrica. En los modelos del tipo "bottom-up" (o sea, aquellos sin una obvia inmersión en ST) que son los que consideramos a lo largo de esta tesis, siempre nos quedamos con el sector de gravedad clásica de Einstein más campos de materia (para esto hemos usado las aproximaciones de curvatura débil y bajas energías). Esto sugiere que las construcciones de BH podrían sernos útiles para entender la dinámica de los HTSC. Pero los BH que estudiamos no nos dicen demasiado acerca de la dinámica microscópica del proceso de formación de pares de Cooper en los HTSC. En esta descripción la gravedad no es mas que una teoría de GL (sección (3.2), aunque estos BH superconductores pueden ser vistos como un primer paso para aproximar a los HTSC holográficamente. AdS/CFT nos da la posibilidad de poder obtener resultados no perturbativos que hasta ahora eran impensados. Estos resultados 
podrían originar nuevas ideas acerca de cómo se comportan estos sistemas y ser aplicadas a los materiales reales. Por otro lado, otros tipo de superconductores holográficos denominados de onda $p$ y de onda $p+i p$ son los que fueron estudiados en profundidad en esta tesis y se detallan en las secciones (5) y (6).

\subsection{Metales extraños holográficos}

La forma más directa de detectar la presencia de una FS es a través del propagador fermiónico, que como vimos en la sección (3.7), captura el pico infinito de la cuasi-partícula para $k=k_{F}$. Dados los orígenes supersimétricos de la correspondencia $A d S / C F T$, los fermiones aparecen en forma natural pero presentan profundas diferencias con los operadores bosónicos.

Antes de introducir fermiones en la teoría especificaremos la geometría donde luego introduciremos los fermiones. Consideramos una acción del tipo:

$$
S=\frac{1}{2 \kappa^{2}} \int d^{4} x \sqrt{-g}\left(R-\frac{6}{R^{2}}-\frac{R^{2}}{g_{F}^{2}} F_{\mu \nu} F^{\mu \nu}\right)
$$

Las ecuaciones de movimiento que se obtienen de (180) tienen como solución un AdSBH cargado o RNAdSBH 11:

$$
d s^{2}=\frac{r^{2}}{L^{2}}\left(-f d t^{2}+d \vec{x}^{2}\right)+L^{2} \frac{d r^{2}}{r^{2} f}
$$

donde:

$$
f=1+\frac{Q^{2}}{r^{4}}-\frac{M}{r^{3}} \quad ; \quad A_{0}=\mu\left(1-\frac{r_{0}}{r}\right)
$$

donde $r_{0}$ es el horizonte del BH definido como $f\left(r_{0}\right)=0$, y $\mu \equiv \frac{g_{F} Q}{L^{2} r_{0}}$ puede ser identificado como el potencial químico de la teoría del borde (de manera análoga al caso de superconductores holográficos). Para trabajar tanto teórica como númericamente es conveniente usar cantidades adimensionales. Mediante los rescaleos:

$$
r \rightarrow r_{0} r \quad ; \quad(t, \vec{x}) \rightarrow \frac{R^{2}}{r_{0}}(t, \vec{x}) \quad ; \quad A_{0} \rightarrow \frac{r_{0}}{R^{2}} A_{0} \quad ; \quad M \rightarrow M r_{0}^{3} \quad ; \quad Q \rightarrow Q r_{0}^{2}
$$

podemos fijar $L=r_{0}=1$. La temperatura del BH se puede calcular igual que como se mostró en la sección $(2.7 .2)$ :

$$
T=\frac{1}{4 \pi}\left(3-Q^{2}\right)
$$

El límite de temperatura cero corresponde a $Q^{2}=3$.

En esta sección trabajaremos con los fermiones en el límite de prueba, en el cual ellos no afectan la métrica y el campo de Maxwell. Los fermiones son descriptos por un campo de Dirac en un espacio curvo en presencia de un campo electromagnético externo. La acción viene dada por:

$$
S_{\text {Dirac }}=-i \int d^{4} x \sqrt{-g}(\bar{\Psi}(\not D-m) \Psi)
$$

donde $\not D=\Gamma^{\mu} D_{\mu}, \bar{\Psi}=\Psi^{\dagger} \Gamma^{t}$ y $D_{M}=\partial_{M}+\frac{1}{4} \omega_{a b M} \Gamma^{a b}-i q A_{M}$. Ya que la ecuación de Dirac es proporcional a las ecuaciones de movimiento, la acción on-shell resulta ser cero. Esto refleja la naturaleza cuántica inherente de los fermiones, por lo cual ellos nunca influyen en la aproximación de punto silla. Por lo tanto pareciese que la función de partición es independiente de la condición de borde y no podremos reproducir ninguna de las funciones de correlación de la teoría de campos que vive en el borde. Esto no es así, veamos porqué. Variemos la acción (185):

$$
\delta S_{\text {Dirac }}=-i \int d^{4} x(\delta \bar{\Psi} \not D \Psi+\not D(\bar{\Psi} \delta \Psi)-\not D \bar{\Psi} \delta \Psi-m \delta(\bar{\Psi} \Psi))
$$

El término que contiene una derivada total se puede escribir como:

$$
-i \int d^{4} \sqrt{-g} \not D(\bar{\Psi} \delta \Psi)=-i \int_{r=r_{\epsilon}} d^{3} x \sqrt{-h} \bar{\Psi} \delta \Psi=-i \int_{r=r_{\epsilon}} d^{3} x \sqrt{-h}\left(\bar{\Psi}_{+} \delta \Psi_{-}+\bar{\Psi}_{-} \delta \Psi_{+}\right)
$$

Por lo tanto la variación (186) se puede escribir como:

$$
\delta S_{\text {Dirac }}=-i \int d^{4} x(\delta \bar{\Psi}(\not D-m) \Psi-(\not D-m) \bar{\Psi} \delta \Psi)-i \int_{r=r_{\epsilon}} d^{3} x \sqrt{-h}\left(\bar{\Psi}_{+} \delta \Psi_{-}+\bar{\Psi}_{-} \delta \Psi_{+}\right)
$$

\footnotetext{
${ }^{11}$ Respecto del ansatz (159) hemos rescaleado $f \rightarrow r^{2} / L^{2} f$; la diferencia entre (182) y (160) radica en que aquí estamos considerando el efecto de los campos de materia sobre la métrica (salimos del límite de prueba), origen del término $Q^{2} / r^{4}$.
} 
Vemos que la $\delta S_{\text {Dirac }}$ on-shell no resulta ser cero por la presencia del término de borde. Para cancelar el mismo se debe agregar a la acción el termino de borde (187), cuya variación cancele el segundo término en (188). El término apropiado es:

$$
S_{b d y}=i \int_{r=r_{\epsilon}} d^{3} x \sqrt{-h} \bar{\Psi}_{+} \Psi_{-}
$$

Por lo tanto la acción total a considerar es:

$$
S_{\text {fermion }}=-i \int d^{4} x \sqrt{-g}(\bar{\Psi}(\not D-m) \Psi)+i \int_{r=r_{\epsilon}} d^{3} x \sqrt{-h} \bar{\Psi}_{+} \Psi_{-}
$$
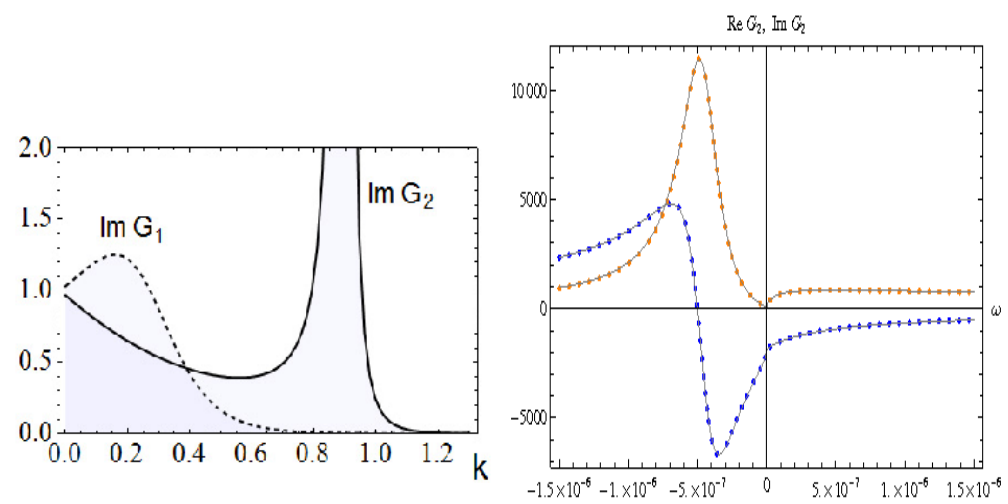

Figura 22: Izquierda: Se muestra $\operatorname{Im}\left(G_{11}\right)$ curva punteada y $\operatorname{Im}\left(G_{22}\right)$ curva solida como función de k para $\omega=-0.001$ y $(m=0, q=1)$. En $\operatorname{Im}\left(G_{22}\right)$ se ve claramente un pico cerca de $k_{F} \approx 0.9185$. Derecha: Se muestra la parte real (curva azul) e imaginaria (curva naranja) de $G_{22}$ para $k=0.9<k_{F}$. En $\operatorname{Im}\left(G_{22}\right)$ para $\omega<0$ hay un pico de cuasi-partícula. Para $\omega>0$ una pequeña loma está presente. Conforme ke aproxima a $k_{F}$ el pico y la loma se aproximan a cero y sus pesos tienden a infinito.

Las ecuaciones de movimiento, la imposición de las condiciones de contorno y como resultado la obtención de la función de Green retardada asociada a la acción (190) se analizan con detalle en la sección (7). Aquí se presentarán solo algunas ecuaciones e ideas relevantes acerca del cálculo de la función de Green. Notamos primero que el campo de Dirac se acopla a un operador espinorial $\hat{O}(t, \vec{x})$ de la QFT del borde. La estructura asintótica de las soluciones de (190) es algo distinta ya que las ecuaciones diferenciales para $\Psi$ son de primer orden, en particular el número de componentes del operador $\hat{O}$ es la mitad del número de componentes de $\Psi$, o sea, mientras $\Psi$ tiene cuatro componentes, $\hat{O}$ tiene dos, como es de esperar en un espacio-tiempo de tres dimensiones (ver [80]). El resultado de la función de Green es:

$$
G_{\alpha}(\omega, k)=\frac{b_{\alpha}(\omega, k)}{a_{\alpha}(\omega, k)}, \alpha=1,2
$$

donde $a_{\alpha}$ y $b_{\alpha}$ son los coeficientes de expansión en el borde como en (96). Una relación útil es: $G_{1}(\omega, k)=$ $G_{2}(\omega,-k)$, la cual permite concentrarnos en $G_{2}(\omega, k)$. Vemos que cuando $a_{\alpha}(\omega, k)=0$ tenemos un polo en la función de Green que indica una divergencia en $\operatorname{Im}_{2}(\omega, k)$. Definimos el momento de Fermi $k_{F}$ como el valor de $k$ a $\omega=0$ (energía de excitación nula) tal que $\operatorname{Im} G_{2}\left(0, k_{F}\right)$ tiene una divergencia. De esta forma podemos calcular los momentos de Fermi numéricamente para diferentes parámetros. La figura (22)(izquierda) muestra una divergencia en $\operatorname{Im} G_{2}$ para $\omega \approx 0$ en un valor especifico de $k$, señalando la ubicación del momento de Fermi $k_{F}$. De la figura $(22)$ (derecha) se ve la presencia de un pico agudo para $\omega<0$ y en la región $\omega>0$ una loma. Esto parece indicar la presencia de un pico de cuasi-partícula, en primera instancia. Si se grafican diferentes gráficas de este estilo, para diferentes valores de $\mathrm{k}$ cercanos a $k_{F}$ y anotamos el valor de $\omega$ para el cual ocurre este máximo, denotado como $\omega_{*}\left(k_{\perp}\right)$, podemos ir siguiendo este pico y encontrar una relación funcional entre $\omega_{*}\left(k_{\perp}\right)$ y $k_{\perp} \equiv k-k_{F}$ para $k_{\perp} \rightarrow 0^{-}$. Se encuentra que:

$$
\omega_{*}\left(k_{\perp}\right) \sim k_{\perp}^{z} \quad, \quad z=2.09 \pm 0.01
$$

y el peso del máximo de la función espectral escalea como:

$$
\rho=\operatorname{Im} G_{22}\left(\omega_{*}\left(k_{\perp}\right), k_{\perp}\right) \sim k_{\perp}^{-\alpha} \sim \omega_{*}^{-\alpha / z} \quad, \quad \alpha=1.00 \pm 0.01
$$


En un líquido de Fermi-Landau los exponentes toman los valores $z=\alpha=1$.

Es posible hallar la función de Green en una forma semi-analítica para frecuencias pequeñas $\omega<<\mu$ usando el método de pareo (que se explica en detalle en el ápendice 7.8). El uso de esta técnica se debe a que a altas temperaturas $(T>>\mu)$ la función de Green $G_{R}(\omega, k)$ es analítica cerca de $\omega=0$ entonces es posible hacer una expansión del espinor alrededor de $\omega$, pero para temperaturas pequeñas $T<<\mu$ y bajas frecuencias $\omega / \mu$ la expansión es delicada ya que en la ecuación de movimiento para el espinor aparecen términos de la forma $(\omega / f)$ donde cerca del horizonte tenemos $f\left(r_{0}\right) \rightarrow 0$ no importa que tan chico sea $\omega$. El resultado final para la función de Green resulta ser de la forma:

$$
G_{R}(\omega, k)=\frac{b_{0}^{+}(k)+b_{1}^{+}(k) \omega+\cdots+\left(b_{0}^{-}(k)+\ldots\right) \mathcal{G}_{k}(\omega)}{a_{0}^{+}(k)+a_{1}^{+}(k) \omega+\cdots+\left(a_{0}^{-}(k)+\ldots\right) \mathcal{G}_{k}(\omega)}
$$

donde los coeficientes $a_{ \pm}$en (194) tienen una expansión analítica en $\omega$ :

$$
a_{+}(\omega, k)=a_{+}^{(0)}(k)+a_{+}^{(1)}(k) \omega+a_{+}^{(2)}(k) \omega^{2}+\ldots
$$

de igual forma para $b_{ \pm}(\omega, k)$. Estamos interesados en la función de Green cerca de la FS ya que los fenómenos que ocurren en las cercanías de la FS dominan las propiedades termodinámicas a bajas temperaturas. Cerca de la FS y para frecuencias bajas tenemos la siguiente expansión:

$$
G_{R}(\omega, k) \approx \frac{h_{1}}{k-k_{F}-\frac{1}{v_{F}} \omega-\Sigma(\omega)} \quad ; \quad \Sigma(\omega)=h \mathcal{G}_{k}(\omega)=h c\left(\nu_{k_{F}}\right) \omega^{2 \nu_{k_{F}}}
$$

con:

$v_{F}=\frac{\partial_{k} a_{+}^{(0)}\left(k_{F}\right)}{a_{+}^{(1)}\left(k_{F}\right)} \quad ; \quad h_{1}=\frac{b_{+}^{0}\left(k_{F}\right)}{\partial_{k} a_{+}^{(0)}\left(k_{F}\right)} \quad ; \quad h=-\frac{a_{-}^{(0)}\left(k_{F}\right)}{\partial_{k} a_{+}^{(0)}\left(k_{F}\right)} \quad ; \quad c(\nu)=2^{\nu_{k}} \frac{\Gamma\left(-2 \nu_{k}\right) \Gamma\left(\frac{1}{2}-\nu_{k}-i q e_{d}\right)}{\Gamma\left(2 \nu_{k}\right) \Gamma\left(\frac{1}{2}+\nu_{k}-i q e_{d}\right)}$

Por lo tanto podemos ver que la auto-energía $\Sigma(\omega)$ solo depende de la frecuencia, y es caracterizada por un exponente no trivial $\nu_{k_{F}}$ que viene dado por:

$$
\nu_{k}=\sqrt{m_{k}^{2} L_{2}^{2}-q^{2} e_{d}^{2}} \quad ; \quad m_{k}^{2}=\frac{k^{2} L^{2}}{r_{*}^{2}}+m^{2}
$$

donde: $L_{2}=\frac{1}{\sqrt{d(d-1)}} L, e_{d}=\frac{g_{F}}{\sqrt{2 d(d-1)}} \mathrm{y} r_{*}^{d-1}=\sqrt{\frac{d-2}{d}} Q$.

La variación de $\nu_{k_{F}}$ como función de $q$ y $m$ se muestra en la figura (4.2)(derecha). Los polos de (196) son determinados vía:

$$
k-k_{F}-\frac{1}{v_{F}} \omega_{c}-\Sigma\left(\omega_{c}\right)=0 \quad \longrightarrow \quad \omega_{c}(k)=\omega_{*}(k)-i \Gamma(k)
$$

La parte real de $\omega_{c}(k), \omega_{*}(k)$, define la relación de dispersión de las cuasi-partículas, mientras que su parte imaginaria $\Gamma(k)$ define el ancho de una excitación, el cual resulta ser proporcional a la parte imaginaria de $\mathcal{G}_{k}(\omega)$. Por lo tanto vemos que dependiendo de los valores de $\nu_{k_{F}}$ la física sera diferente.

- Caso $\nu_{k_{F}}>1 / 2$ : líquido de Fermi regular.

Si $\nu_{k_{F}}>1 / 2$ el término líneal de (196) domina sobre el termino de la auto-energía $\omega^{2 \nu_{k_{F}}}$ (recordemos que estamos a frecuencias bajas). Luego la expresión para el movimiento del polo sera

$$
\omega_{c}(k)=v_{F}\left(k-k_{F}\right)-v_{F} h c\left(\nu_{k_{F}}\right) v_{F}\left(k-k_{F}\right)^{2 \nu_{k_{F}}}
$$

Tenemos entonces una relación de dispersión líneal $\omega_{*}=v_{F}\left(k-k_{F}\right)$ con ancho $\Gamma \sim \omega^{2 \nu_{k_{F}}}$ que es siempre mucho mas pequeño que la energía $\omega_{*}$. Por lo tanto tenemos cuasi-partículas con una gran vida media, que cualitativamente no son muy diferentes de las excitaciones de un líquido de Fermi, excepto que ahora el ancho satisface $\Gamma \sim \omega^{2 \nu_{k_{F}}}$ en lugar de $\Gamma \sim \omega^{2}$ como en el líquido de Fermi-Landau.

- Caso $\nu_{k_{F}}<1 / 2$ : líquido de Fermi singular.

En este caso la contribución de $\Sigma(\omega)$ domina sobre el término lineal. La solución a (199) es ahora:

$$
\omega_{c}(k)=\mu_{*}\left(\frac{\left(k-k_{F}\right)^{1 / 2 \nu}}{h c\left(\nu_{k_{F}}\right)}\right)
$$


Como $c\left(\nu_{k_{F}}\right)$ es complejo este polo tiene parte real y imaginaria. La relación de dispersión es ahora no líneal, $\omega_{*} \sim\left(k-k_{F}\right)^{1 / 2 \nu_{k}}$, y el ancho también tiene el mismo comportamiento: $\Gamma(k) \sim\left(k-k_{F}\right)^{1 / 2 \nu_{k_{F}}}$. Por lo tanto:

$$
\frac{\Gamma}{\omega_{*}(k)} \sim \text { constante }
$$

El residuo del polo es

$$
Z=-\frac{\omega_{c} h_{1}}{2 \nu\left(k-k_{F}\right)} \sim\left(k-k_{F}\right)^{\frac{1}{2 \nu}-1}
$$

mientras que el residuo del polo para $k \rightarrow k_{F}$ se anula. El hecho de que el ancho de la excitación sea del orden de su energía y que el residuo desaparezca indica que la misma no puede considerarse como una cuasi-partícula. Tenemos un ejemplo donde el sistema tiene una superficie de Fermi bien definida pero no existe el concepto de cuasi-partícula.

- Caso $\nu_{k_{F}}=1 / 2$ : líquido de Fermi marginal.

En este caso ambos términos en (196) son proporcionales a $\omega$. Básicamente tenemos un cero simple en $v_{F}$, y $c\left(\nu_{k_{F}}\right)$ en la auto-energía $\Sigma(\omega)$ tiene un polo simple para $\nu_{k_{F}}=1 / 2$; las dos divergencias se cancelan y dejan un término finito $\omega \ln \omega$ con $\tilde{c}_{1}$ real, más otro término $c_{1} \omega$ complejo, ver [68]:

$$
G_{R}(\omega, k) \approx \frac{h_{1}}{k-k_{F}+\tilde{c}_{1} \omega \ln \omega+c_{1} \omega}
$$

Esta función de Green es exactamente la postulada para la función respuesta de un metal extraño discutida en la sección (3.7). Acá vemos que notablemente surge de cálculos holográficos. Notemos que el ancho viene dado por $\Gamma \sim \omega$, dando lugar a que el residuo del polo escalee como $Z \sim 1 / \ln \left(k-k_{F}\right)$ y así $Z^{-1}$ diverge logarítmicamente.
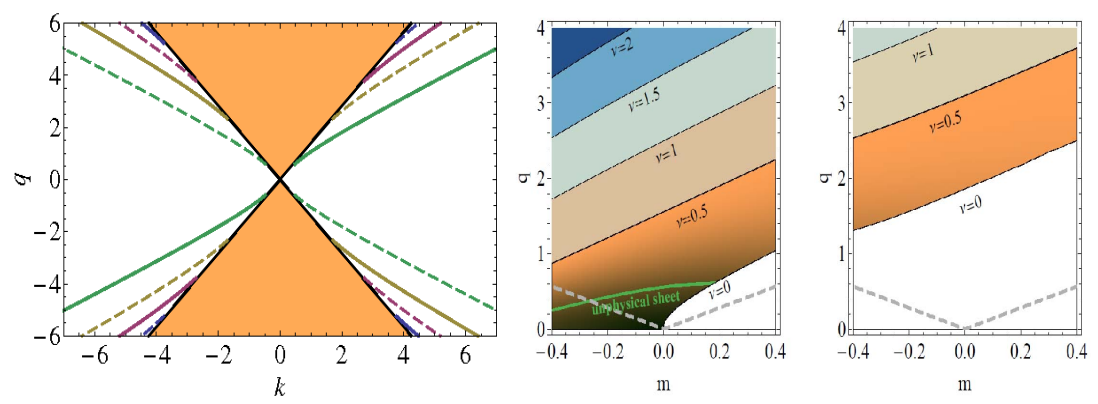

Figura 23: Izquierda: Las líneas sólidas representan los valores de $k_{F}$ para distintos valores de $q$. La zona sombreada representa la región donde el parámetro $\nu_{k}$ es complejo. Las líneas punteadas corresponden a lo mismo pero en la cuantización alternativa. El gráfico resulta simétrico frente al intercambio de $(q, k) \rightarrow$ $(-q,-k)$. Derecha: Se muestra como se distribuye $\nu_{k_{F}}$ en el plano $m-q$. La zona blanca corresponde a una región donde no hay superficie de Fermi. Vemos que en la región $\nu_{k_{F}}<1 / 2$ no hay cuasi-partículas con gran vida media. En el resto del espacio de parámetros hay cuasi-partículas con gran vida media.

\subsection{Estrella de electrones}

Esta sección se basa en el artículo [115].

Comenzaremos haciéndonos la siguiente pregunta: ¿cuál es el sistema físico de materia fermiónica compactada debido a la gravedad? La respuesta es una estrella, como en el caso de una estrella de neutrones en Astrofísica. Para altas densidades es posible aproximar el gas de Fermi como un fluido perfecto, aproximación conocida como de Thomas-Fermi o de fluido. Consiste en tomar el límite donde el número de fermiones por radio de $A d S$ L, es muy grande (infinito) respecto de la energía de Fermi, que permanece fija. Dicho de otro modo, la escala de longitud fijada por las variaciones de los campo fermiónicos es muy pequeña comparada con la escala de longitud fijada por la curvatura del espacio, es decir, $E_{F} L>>1$. De esto se desprende que cada fermión ve localmente un espacio-tiempo plano y por lo tanto podemos usar las expresiones usuales para un gas de Fermi ideal. El efecto de la gravedad es solo notable si comparamos diferentes puntos. La métrica juega el rol de un potencial de captura, el efecto de la curvatura se resume en un potencial químico 
local al que está sometido efectivamente el fluido de fermiones. Por lo tanto en la aproximación de fluido la acción de Dirac es reemplazada por una acción efectiva:

$$
S_{\Psi}^{e f f}=\int d^{d+1} x \sqrt{-g} p_{f}
$$

donde $p_{f}$ es la presión del fluido de fermiones.

Comencemos recordando la descripción usual de un fluido perfecto en espacio plano. Los parámetros que caracterizan a éste son la presión $p_{f}$, la densidad de energía $\rho_{f}$ y la densidad de carga $\sigma_{f}$. Estas variables están dadas por:

$$
\begin{gathered}
\rho_{f}=\int_{E_{\min }}^{E_{\max }} E g(E) d E=\int_{m}^{\epsilon_{F}=\mu} E g(E) d E \\
\sigma_{f}=\int_{m}^{\epsilon_{F}=\mu} g(E) d E
\end{gathered}
$$

donde $g(E)$ es la densidad de estados. Notemos que estamos integrando desde el mínimo de energía (masa de la partícula) a la energía de Fermi, o sea, el potencial químico a $T=0$. La densidad de estados para un gas de Fermi relativista en $\mathrm{d}+1$ dimensiones es:

$$
g(E)=\beta_{d+1} E\left(E^{2}-m^{2}\right)^{(d-2) / 2} \stackrel{d=3}{\longrightarrow} g(E)=\beta_{4} E \sqrt{E^{2}-m^{2}}
$$

El potencial gran canónico es por definición: $\Omega \equiv F-\mu N=U-T S-\mu N$ donde a $T=0$ se tiene que $\Omega=-p V$, llegando a:

$$
-p_{f}=\rho_{f}-\mu \sigma_{f}
$$

En la aproximación de densidad local el potencial químico es reemplazado por el potencial químico local:

$$
\mu \rightarrow \mu_{\text {local }}=u^{t} A_{t}
$$

donde $u^{t}$ es la componente temporal del tetravector velocidad $u^{\mu}$, normalizado a $u^{\mu} u_{\mu}=-1$. En el marco de referencia del gas:

$$
u^{t}=\frac{1}{\sqrt{-g_{t t}}}
$$

Localmente el fluido ve un espacio plano pero globalmente se tiene en cuenta la gravedad debido a la introducción de este potencial químico local. Las variables del fluido perfecto de fermiones están dados por:

$$
\rho_{f}=\beta_{d+1} \int_{m}^{\mu_{l o c a l}} E g(E) d E \quad ; \quad \sigma_{f}=\beta_{d+1} \int_{m}^{\mu_{l o c}} g(E) d E \quad ; \quad-p_{f}=\rho_{f}-\mu_{l o c} \sigma
$$

El efecto de los fermiones sobre la métrica en la aproximación de fluido es capturado por el tensor de energía-momento del fluido perfecto, que aparece como fuente de la ecuación de Einstein, a saber:

$$
T_{\mu \nu}^{f}=\left(\rho_{f}+p_{f}\right) u_{\mu} u_{\nu}+p_{f} g_{\mu \nu}
$$

La acción a considerar contiene siguiente tipo de materia:

$$
S=\int d^{4} x \sqrt{-g}\left(\frac{1}{2 \kappa^{2}}(R-2 \Lambda)-\frac{1}{4 e^{2}} F_{\mu \nu} F^{\mu \nu}-p\left(\mu_{l o c}\right)\right)
$$

cuyas ecuaciones de movimiento son

$$
\begin{gathered}
R_{\mu \nu}+\left(\Lambda-\frac{1}{2} R\right) g_{\mu \nu}=\kappa^{2} T_{\mu \nu}=\kappa^{2}\left(T_{\mu \nu}^{A}+T_{\mu \nu}^{f}\right) \\
\nabla_{\mu} F^{\nu \mu}=e^{2} J^{\nu}=e^{2} \sigma u^{\nu} \\
\nabla_{\mu} T^{\mu \nu}=0
\end{gathered}
$$

Propondremos un ansatz para la métrica tal que a altas energías la teoría cuántica dual asociada sea invariante de escala como vimos en la sección (2.7.3):

$$
d s^{2}=-L^{2}\left(f(r) d t^{2}+g(r) d r^{2}+\frac{1}{r^{2}}\left(d x^{2}+d y^{2}\right)\right) \quad ; \quad A=\frac{e L}{\kappa} h d t
$$


donde $f(r)$ y $g(r)$ son funciones desconocidas. Imponiendo que el espacio para $r \rightarrow 0$ sea asintóticamente $A d S$ tenemos que para gran $r: f(r) \rightarrow 1 / r^{2}$ y $g(r) \rightarrow 1 / r^{2}$. Es conveniente trabajar con cantidades adimensionales; notando que:

$$
[p]=[\rho]=[\text { longitud }]^{-4} \quad ; \quad[\sigma]=[\text { longitud }]^{-3} \quad ; \quad[\kappa]^{2}=[\text { longitud }]^{2} \quad,
$$

podemos definir las variables adimensionales:

$$
\hat{p}=L^{2} \kappa^{2} p \quad ; \quad \hat{\rho}=L^{2} \kappa^{2} \rho \quad ; \quad \hat{\sigma}=\frac{1}{e L^{2} \kappa} \sigma
$$

Las ecuaciones de movimiento que se obtienen toman la forma:

$$
\begin{gathered}
\hat{p}^{\prime}+(\hat{p}+\hat{\rho}) \frac{f^{\prime}}{2 f}-\frac{h^{\prime} \hat{\sigma}}{\sqrt{f}}=0 \\
\frac{1}{r}\left(\frac{f^{\prime}}{f}+\frac{g^{\prime}}{g}+\frac{4}{r}\right)+(\hat{p}+\hat{\rho}) g=0 \\
\frac{f^{\prime}}{f r}-\frac{\left(h^{\prime}\right)^{2}}{2 f}+g(3+\hat{p})-\frac{1}{r^{2}}=0 \\
h^{\prime \prime}+\frac{r h^{\prime} g(\hat{p}+\hat{\rho})}{2}-g \sqrt{f} \hat{\sigma}=0
\end{gathered}
$$

Las cantidades termodinámicas adimensionales vendrán dadas ahora por:

$$
\begin{gathered}
\hat{\rho}=\hat{\beta} \int_{\hat{m}}^{\frac{h}{\sqrt{f}}} \epsilon^{2} \sqrt{\epsilon^{2}-\hat{m}^{2}} d \epsilon \\
\hat{\sigma}=\hat{\beta} \int_{\hat{m}}^{\frac{h}{\sqrt{f}}} \epsilon \sqrt{\epsilon^{2}-\hat{m}^{2}} d \epsilon \\
-\hat{p}=\hat{\rho}-\frac{h}{\sqrt{f}} \hat{\sigma}
\end{gathered}
$$

donde:

$$
\hat{\beta} \equiv \frac{e^{4} L^{2}}{\kappa^{2}} \beta \quad ; \quad \hat{m}^{2} \equiv \frac{\kappa^{2}}{e^{2}} m^{2}
$$

Los ansatz (225), (226) y (227) determinan tres de las funciones a encontrar. La ecuación (221) es en efecto la ecuación de conservación de la energía y es verificada por (225), (226) y (227), por lo cual las cuatro ecuaciones (221), (222), (223) y (224) se reducen a

$$
\begin{gathered}
\frac{1}{r}\left(\frac{f^{\prime}}{f}+\frac{g^{\prime}}{g}+\frac{4}{r}\right)+(\hat{p}+\hat{\rho}) g=0 \\
\frac{f^{\prime}}{f r}-\frac{\left(h^{\prime}\right)^{2}}{2 f}+g(3+\hat{p})-\frac{1}{r^{2}}=0 \\
h^{\prime \prime}+\frac{r h^{\prime} g(\hat{p}+\hat{\rho})}{2}-g \sqrt{f} \hat{\sigma}=0
\end{gathered}
$$

El fluido perfecto más la gravedad se reduce a las ecuaciones de un fluido de fermiones autogravitantes denominado estrella de electrones.

En la región IR, que en nuestras coordenadas se corresponde a $r \rightarrow \infty$ se encuentra una escala emergente tipo Lifshitz, es decir, la geometría en el interior de esta estrella es del tipo Lifshitz determinado por un exponente dinámico crítico $z$. En efecto, tal métrica es una solución exacta en el IR de las ecuaciones de movimiento (229), (230) y (231). La métrica y la función de Maxwell toman la forma

$$
f=\frac{1}{r^{2 z}} \quad ; \quad g=\frac{g_{\infty}}{r^{2}} \quad ; \quad h=\frac{h_{\infty}}{r^{z}}
$$

Lo siguiente que haremos es seguir al flujo de renormalización, es decir, comenzar desde el punto fijo en el infrarojo dado por la métrica de Lifshitz y perturbarla para encontrar la solución para todo $r$, es decir queremos ver hasta donde se extiende la solución en el UV. Se propone un ansatz de la forma: 


$$
f=\frac{1}{r^{2 z}}\left(1+f_{1} r^{\alpha}+\ldots\right) \quad ; \quad g=\frac{g_{\infty}}{r^{2}}\left(1+g_{1} r^{\alpha}+\ldots\right) \quad ; \quad h=\frac{h_{\infty}}{r^{z}}\left(1+h_{1} r^{\alpha}+\ldots\right)
$$

Sustituyendo las expansiones de arriba en las ecuaciones de movimiento se encuentran tres soluciones para el parámetro $\alpha$ :

$$
\alpha_{0}=2+z \quad ; \quad \alpha_{ \pm}=\frac{2+z}{2} \pm \frac{\sqrt{9 z^{3}-21 z^{2}+40 z-28-\hat{m}^{2} z(4-3 z)^{2}}}{2 \sqrt{\left(1-\hat{m}^{2}\right) z-1}}
$$

El exponente $\alpha_{-}$es negativo y por lo tanto es el que debemos considerar. La presencia de los modos $\alpha_{0}$ y $\alpha_{+}$ deben ser puestos a cero para obetener una solución regular en el IR, ya que se debe integara numéricamente desde el IR hasta el borde. Dado el valor del exponente $\alpha_{-}$se pueden determinar los coeficientes $g_{1}$ y $h_{1}$ y todos los coeficientes más altos en términos de $f_{1}$ el cual en principio no esta determinado. No obstante $f_{1}$ puede ser elegido para que tome cualquier valor mediante un cambio de variables de las coordenadas $t, r, \vec{x}$, aunque el signo queda sin ser definido. Se elige el signo de $f_{1}$ para que cuando $r \rightarrow 0$ se obtenga la solución de un $\mathrm{BH}$ cargado en $A d S$. Para lograrlo se tomara el valor $f_{1}=-1$. Luego podemos proceder a integrar numéricamente hasta pequeños valores de $r$. Se llega a una zona donde para algún radio especifico $r_{s}$ la estrella termina y la presión, densidad de carga y densidad de energía tienden a cero.

La solución que se encuentra se muestra en la figura (24), donde se puede ver como varían las variables termodinámicas del fluido de fermiones desde sus valores constantes de Lifshitz a grandes $r$ hasta el radio de la estrella $r=r_{s}$. Cabe notar que a diferencia de estrellas reales, la región IR del espacio-tiempo a grandes valores de $r$ tiene un volumen infinito en la dirección radial. El borde de la estrella ocurre cuando el potencial químico local no es suficientemente grande para poblar el mar de Fermi. Este valor viene dado por $m=\frac{h\left(r_{s}\right)}{\sqrt{f\left(r_{s}\right)}}$. Estos perfiles radiales son similares a los perfiles del fluido de una estrella de neutrones.

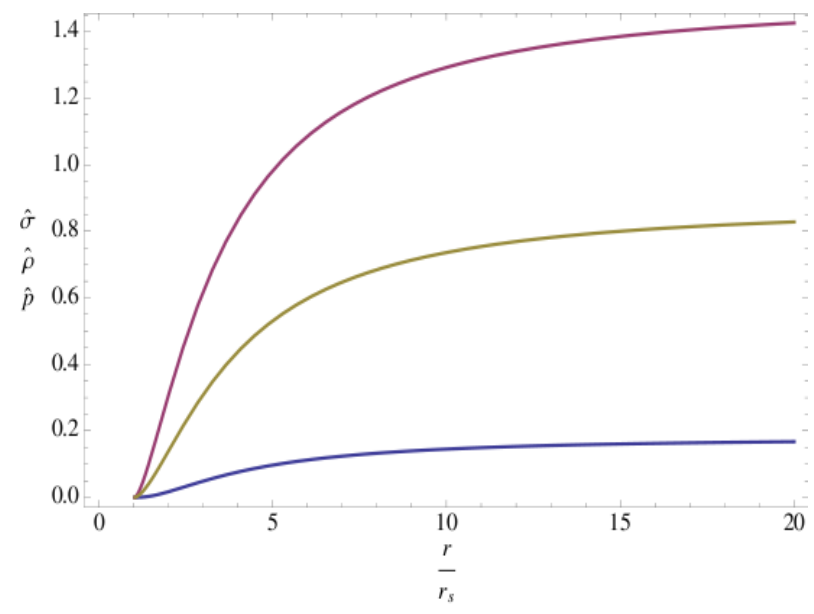

Figura 24: Desde arriba hacia abajo se corresponden a las soluciones de la presión, energía y densidad de carga para una estrella de electrones con $z=2$ y $m=0.36$ (correspondiente a $\beta \approx 20$ ). El borde de la estrella es $r=r_{s}$. El borde del espacio-tiempo es $r=0$ mientras que la profundidad del IR es $r \rightarrow \infty$.

Para explicar más acerca de como es posible la formación física de esta estrella, nos haremos la siguiente pregunta: ¿cuál es el destino final de un agujero negro cargado en presencia de fermiones?. En presencia de fermiones los BH cargados son inestables a descargarse llevando la geometría a quedarse sin horizonte, pues los campos de materia apantallarán y descargarán al agujero negro. La explicación de este proceso es la siguiente: la producción de pares de Schwinger de fermiones ocurre cerca del horizonte del BH (que geométricamente es $\left.A d S_{2} \times R^{2}\right)$ si la masa de los fermiones es suficientemente baja, $(L m)^{2} \leq \gamma^{2} \equiv(e L / \kappa)^{2}$. Esta inestabilidad no conduce a la ocupación macroscópica de un estado, como en el caso de los superconductores holográficos (debido a que los fermiones cumplen la estadística de Fermi-Dirac) sino que se espera que la producción de pares conduzca a una neutralización del BH y un mar de Fermi aparezca fuera del horizonte, el cual contendrá toda la carga. Por lo tanto podemos concluir que el estado que describe esta estrella de electrones a $T=0$ y con entropia nula, es el de un sistema de fermiones fuertemente acoplado que forman una superficie de Fermi. 


\title{
5 Transiciones holográficas en presencia de campo un campo de Higgs en un agujero negro cargado no abeliano
}

\begin{abstract}
Resumen
Encontramos soluciones de una teoría de gravedad-Yang-Mills-Higgs en $3+1$ dimensiones que representan un espacio anti-de Sitter con un agujero negro cargado con parcial/completa ruptura de simetría de calibre. Aplicamos la correspondencia $A d S / C F T$ para estudiar el régimen de acoplamiento fuerte de una teoría cuántica de campos en $2+1$ dimensiones a temperatura y potencial químico finito, la cual experimenta una transición de fase exhibiendo la condensación de un campo vectorial compuesto y cargado bajo una temperatura crítica $T_{c}$ presumiblemente describiendo superconductores del tipo $p+i p / p$. En el caso de un superconductor del tipo $p+i p$ hemos encontrado que las transiciones de fase son siempre de segundo orden pero para los superconductores de tipo $p$, determinamos la existencia de un valor crítico $\alpha_{c}$ del acoplamiento gravitacional (para un valor fijo v.e.v del parámetro $\hat{m}_{W}$ ) más allá del cual la transición se vuelve de primer orden. También hemos encontrado soluciones correspondientes a $T=0$ tales estados son descriptos por geometrías de tipo domain wall (pared de dominio) que interpolan entre espacios $A d S_{4}$ con diferentes velocidades de la luz y para un dado valor de $\hat{m}_{W}$ existe un valor crítico del acoplamiento que no es posible superar. El comportamiento del parámetro de orden como función del acoplamiento gravitacional cerca del acoplamiento crítico sugiere la presencia de una transición cuántica de segundo orden. Finalmente estudiamos la dependencia de la solución en presencia de una constante de acoplamiento debida al Higgs y encontramos la existencia de un valor crítico a partir del cual no se encuentra una fase condensada.
\end{abstract}

\subsection{Introducción}

Un agujero negro cargado introduce una densidad de carga/potencial químico y una temperatura en una teoría cuántica de campos (QFT) definida sobre el borde usando la correspondencia calibre/gravedad. Este marco permite estudiar las transiciones de fase y construir diagramas de fase en el espacio parámetros.

El modelo mas simple es provisto por una teoría de Einstein-Maxwell acoplada a un campo escalar cargado que, en el marco de la correspondencia AdS/CFT, es dual a un operador escalar que lleva la carga de la simetría global $U(1)$. Ha sido probado que una solución de un agujero negro cargado, interpretada como una fase no condensada, se convierte en inestable y desarrolla pelo escalar a bajas temperaturas rompiendo la simetría $U(1)$ cerca del horizonte del agujero negro [11] [12]. Este fenómeno en general puede ser interpretado como una transición de fase de segundo orden entre la fase conductora y superconductora. Esta interpretación es soportada por el análisis del comportamiento de la conductividad en estas fases [8]. Han sido también estudiadas soluciones de vórtices que describen superconductores holográficos de tipo II [20] [21] [22] y mas recientemente, modelos espacialmente anisotrópicos en modelos abelianos de superconductores [23]. Tan pronto como estos superconductores holográficos de onda $-s$ fueron introducidos, modelos de superconductores holográficos con pelo vectorial, conocidos como superconductores holográficos de onda- $p$ fueron explorados numéricamente primero en [24] y [9] (para un reciente tratamiento, ver [25]). El ejemplo mas simple de superconductor holográfico de onda $p$ puede ser provisto por una teoría de Einstein-YangMills con un grupo de calibre $S U(2)$ y sin campos escalares, donde la simetría de calibre electromagnética es identificada con un subgrupo $U(1)$ de $S U(2)$. Las otras componentes del campo de calibre $S U(2)$ juegan el rol de campos cargados duales a operadores vectoriales cuyo valor de expectación distinto de cero rompe la simetría $U(1)$ llevándonos a una transición de fase en la teoría de campo dual.

Mas recientemente, soluciones de las ecuaciones de campo gravedad-materia donde ambos parámetros de orden, escalares y vectoriales están presentes fueron consideradas: ellos describen sistemas donde existe competición/coexistencia entre diferentes fases [26]-[29].

Soluciones de diones auto-gravitantes de las ecuaciones de Einstein-Yang-Mills-Higgs (EYMH) en el límite BPS y asintóticas a un espacio global AdS fueron construidas tiempo atrás en [31] and [32]. Ellas fueron extendidas a soluciones diónicas de agujeros negros en [83] and [33], donde estas son interpretadas como describiendo los llamados superconductores de onda $p+i p$ a temperatura finita en la fase condensada. La propuesta del presente trabajo es generalizar trabajos previos encontrando soluciones mas generales de EYMH en un espacio asintóticamente $A d S_{4}$ con masa y densidad de carga eléctrica finita, e interpretar estas vía la dualidad calibre/gravedad como describiendo fases de una teoría de campo fuertemente acoplada, y construir los correspondientes diagramas de fases ${ }^{12}$

Mas específicamente, en primer termino comenzaremos revisando el análisis de [83] [33], verificando la existencia de una transición de segundo orden a lo largo de todo el espacio de parámetros. Fue encontrado

\footnotetext{
${ }^{12}$ Consideraremos el ansatz usual de un horizonte plano, los cuales son relevantes en el estudio de los sistemas de materia condensada con invarianza traslacional. Bajo estas circunstancias la densidad de carga magnética de la solución de dion en [83] [33] desaparece
} 
(ver por ejemplo [34]) que algunos sistemas holográficos experimentan una transición de fase de segundo orden como función de la temperatura a una de primer orden, moviendo el acoplamiento gravitacional de los campos sobre la geometría, cuando tal acoplamiento excede cierto valor crítico. Este fenómeno ocurre en superfluidos holográficos cuando la velocidad es suficientemente alta [35] [36], y fue medida en ciertos tipo de superconductores [37] [38] [39]. Hemos encontrado que este tipo de comportamiento en nuestro sistema en el caso anisotropico, encontrando soluciones condensadas y construyendo el diagrama de fase. En segundo lugar computamos las energías libres y encontramos que para cualquier conjunto de valores de los parámetros libres que determinan las soluciones, la fase anisotrópica es siempre energéticamente favorecida sobre la fase isotrópica como fue conjeturado en otros contextos [40] [41] [42]. En tercer lugar analizamos el límite de temperatura nula, caso que no ha sido analizado anteriormente; para acoplamiento gravitacionales suficientemente bajos encontramos soluciones, las cuales espontáneamente rompen la simetría $U(1)$ y tienen entropía nula, con lo cual describen el verdadero estado base del sistema. Para acoplamientos gravitacionales mas altos que el valor crítico la solución desaparece, que es interpretada como una transición de fase de segundo orden. Por último, analizamos el efecto de un potencial de Higgs no nulo sobre el sistema.

\subsection{Superconductores holográficos: gravedad+Yang-Mills+Higgs}

\subsubsection{El modelo}

Consideremos un sistema de la forma: gravedad - Yang - Mills - Higgs en un espacio-tiempo de $3+1$ dimensiones con la siguiente signatura de Minskowski (-+++). Tomaremos $S U(2)$ como el grupo de calibre, con generadores que satisfacen el álgebra

$$
\left[X_{a}, X_{b}\right]=\epsilon_{a b c} X_{c} \quad ; \quad a, b, c=0,1,2 \quad, \quad \epsilon_{012} \equiv+1
$$

y el campo escalar en la representación adjunta $H=H^{a} X_{a}$. La acción completa que se considerara es,

$$
S=S^{(b u l k)}+S^{(G H)}+S^{(c t)}
$$

donde

$$
\begin{aligned}
S^{(\text {bulk })} & =\int_{\mathcal{M}} d^{4} x \sqrt{|g|}\left(\frac{1}{2 \kappa^{2}}\left(R+\frac{6}{L^{2}}\right)-\frac{1}{4 e^{2}} F_{M N}^{a} F^{a M N}\right. \\
& \left.-\frac{1}{2} D^{M} H^{a} D_{M} H^{a}-\frac{\lambda}{4}\left(H^{a} H^{a}-H_{0}{ }^{2}\right)^{2}\right) \\
S^{(G H)} & =\frac{1}{2 \kappa^{2}} \int_{\partial \mathcal{M}} d^{3} x \sqrt{|h|} 2 K
\end{aligned}
$$

donde $\kappa, e$ and $\lambda$ son los acoplamientos gravitacionales, de calibre y escalares respectivamente, $L$ es la escala $A d S$ relacionada a la constante cosmológica negativa a través de $\Lambda=-3 / L^{2}$ y $H_{0}>0$ define el valor de expectación de vacío del campo de higgs (y entonces la condición de borde en el infinito ver debajo en $(248))$.

Como es bien conocido el termino de Gibbons-Hawking $S^{(G H)}$ es necesario para tener un principio variacional bien definido [43], donde $K \equiv \nabla_{a} n^{a}$ es la traza de la curvatura extrínsica, y $h$ and $n$ son la métrica inducida y el vector normal sobre $\partial \mathcal{M}$. El contratérmino en la acción $S^{(c t)}$ sera discutido en la sección 5.4

El campo de esfuerzos $F_{M N}^{a}$ y la derivada covariante $D_{M}$ actúa sobre el triplete de Higgs $H^{a}$ que esta definido como,

$$
F_{M N}^{a} \equiv \partial_{M} A_{N}^{a}-\partial_{N} A_{M}^{a}+\epsilon_{a b c} A_{M}^{b} A_{N}^{c} \quad ; \quad D_{M} H^{a} \equiv \partial_{M} H^{a}+\epsilon_{a b c} A_{M}^{b} H^{c}
$$

Vamos a considerar coordenadas $\left(x^{\mu}, y\right)$ y un ansatz que preserva invarianza traslacional en las coordenadas $\left\{x^{\mu}, \mu=0,1,2\right\}$,

$$
\begin{aligned}
g & =-f(y) A(y)^{2} d x^{0^{2}}+y^{2}\left(c(y)^{2} d x^{1^{2}}+d x^{2^{2}}\right)+L^{2} \frac{d y^{2}}{f(y)} \\
A & =L^{-1}\left(d x^{0} J(y) X_{0}+d x^{1} K_{1}(y) X_{1}+d x^{2} K_{2}(y) X_{2}\right) \\
H & =H_{0} H(y) X_{0}
\end{aligned}
$$

en lo que sigue sera conveniente introducir las constantes de acoplamiento adimensionales

$$
\alpha \equiv \frac{\kappa}{e L} \quad ; \quad \hat{m}_{W} \equiv e H_{0} L \quad ; \quad \lambda_{0} \equiv e^{2} H_{0}{ }^{4} L^{4} \lambda
$$


Las ecuaciónes de movimiento de la gravedad (e.o.m.) derivadas de (237) resultan,

$$
\begin{aligned}
-(y f(y))^{\prime}+3 y^{2} & -y^{2} f(y) \frac{c^{\prime \prime}(y)}{c(y)}-\left(3 y f(y)+\frac{y^{2}}{2} f^{\prime}(y)\right) \frac{c^{\prime}(y)}{c(y)} \\
& =\alpha^{2}\left(\frac{\lambda_{0}}{4} y^{2}\left(H(y)^{2}-1\right)^{2}+f(y) V_{1}+V_{2}+\frac{y^{2}}{2} \frac{J^{\prime}(y)^{2}}{A(y)^{2}}\right. \\
& \left.+\frac{1}{2}\left(\frac{K_{1}(y)^{2}}{c(y)^{2}}+K_{2}(y)^{2}\right)\left(\hat{m}_{W}{ }^{2} H(y)^{2}+\frac{J(y)^{2}}{f(y) A(y)^{2}}\right)\right) \\
y \frac{A^{\prime}(y)}{A(y)}-\frac{A(y)}{2 c(y)}\left(\frac{y^{2} c^{\prime}(y)}{A(y)}\right)^{\prime} & =\alpha^{2}\left(V_{1}+\frac{1}{2}\left(\frac{K_{1}(y)^{2}}{c(y)^{2}}+K_{2}(y)^{2}\right) \frac{J(y)^{2}}{f(y)^{2} A(y)^{2}}\right) \\
\frac{1}{A(y) c(y)}\left(y^{2} f(y) A(y) c^{\prime}(y)\right)^{\prime} & =\alpha^{2}\left(\left(\frac{K_{1}(y)^{2}}{c(y)^{2}}-K_{2}(y)^{2}\right)\left(\frac{J(y)^{2}}{f(y) A(y)^{2}}-\hat{m}_{W}{ }^{2} H(y)^{2}\right)\right. \\
& \left.-f(y)\left(\frac{K_{1}^{\prime}(y)^{2}}{c(y)^{2}}-K_{2}^{\prime}(y)^{2}\right)\right)
\end{aligned}
$$

mientras que las de la materia son,

$$
\begin{aligned}
\frac{c(y)}{A(y)}\left(\frac{f(y) A(y)}{c(y)} K_{1}^{\prime}(y)\right)^{\prime} & =\left(\frac{K_{2}(y)^{2}}{y^{2}}+\hat{m}_{W}^{2} H(y)^{2}-\frac{J(y)^{2}}{f(y) A(y)^{2}}\right) K_{1}(y) \\
\frac{1}{A(y) c(y)}\left(f(y) A(y) c(y) K_{2}^{\prime}(y)\right)^{\prime} & =\left(\frac{K_{1}(y)^{2}}{c(y)^{2} y^{2}}+\hat{m}_{W}{ }^{2} H(y)^{2}-\frac{J(y)^{2}}{f(y) A(y)^{2}}\right) K_{2}(y) \\
\frac{1}{A(y) c(y)}\left(y^{2} f(y) A(y) c(y) H^{\prime}(y)\right)^{\prime} & =\left(\frac{K_{1}(y)^{2}}{c(y)^{2}}+K_{2}(y)^{2}+\frac{\lambda_{0}}{\hat{m}_{W^{2}}} y^{2}\left(H(y)^{2}-1\right)\right) H(y) \\
\frac{f(y) A(y)}{c(y)}\left(\frac{y^{2} c(y)}{A(y)} J^{\prime}(y)\right)^{\prime} & =\left(\frac{K_{1}(y)^{2}}{c(y)^{2}}+K_{2}(y)^{2}\right) J(y)
\end{aligned}
$$

donde hemos definido

$$
V_{1}=\frac{1}{2}\left(\frac{K_{1}^{\prime}(y)^{2}}{c(y)^{2}}+K_{2}^{\prime}(y)^{2}\right)+\frac{\hat{m}_{W}^{2}}{2} y^{2} H^{\prime}(y)^{2} \quad ; \quad V_{2}=\frac{1}{2} \frac{K_{1}(y)^{2} K_{2}(y)^{2}}{y^{2} c(y)^{2}}
$$

Comenzaremos considerando el límite BPS $\lambda_{0}=0$, pero conservando el valor de vacío de Higgs $H_{0}>0$ que sera crucial en nuestras consideraciones. El efecto de un acoplamiento de Higgs finito sera considerado en la sección 6

\subsubsection{Condiciones de borde}

Investigaremos soluciones de agujeros negros cargados los cuales presentan un horizonte en $y=y_{h}$ donde $f\left(y_{h}\right)=$ 0. La temperatura de Bekenstein-Hawking asociada al agujero negro viene dada por,

$$
T_{B H}=\frac{1}{4 \pi L} A\left(y_{h}\right) f^{\prime}\left(y_{h}\right)
$$

El ansatz (y e.o.m) son invariante bajo la trasformación de escala,

$$
\begin{aligned}
\left(x^{0} ; A(y), J(y)\right) & \longrightarrow \\
\left(x^{1} ; c(y), K_{1}(y)\right) & \left.\longrightarrow\left(\frac{x^{0}}{\beta} ; \beta A(y), \beta J(y)\right)\right) \\
\beta^{\prime} & \left.; \beta^{\prime} c(y), \beta^{\prime} K_{1}(y)\right)
\end{aligned}
$$

Estas permiten fijar alguna normalización imponiendo las b.c (condiciones de borde), $A(y), c(y) \stackrel{y \rightarrow \infty}{\longrightarrow} 1$. Las coordenadas $x^{\mu}$ son identificadas con las coordenadas en un espacio-tiempo de Minkowski donde vive la QFT y (244) con su temperatura. Además se tienen otras simetrías de escala

$$
\left(x^{\mu}, y\right) \rightarrow\left(\frac{x^{\mu}}{\gamma}, \gamma y\right) \quad, \quad f(y) \rightarrow \gamma^{2} f(y) \quad, \quad K_{i}(y) \rightarrow \gamma K_{i}(y) \quad, \quad J(y) \rightarrow \gamma J(y)
$$

si $y_{h} \neq 0$ podemos fijar $y_{h}=1^{13}$

\footnotetext{
${ }^{13}$ Este es el caso excepto cuando consideramos en límite de temperatura cero. Cuando la back-reaction no es tomada en cuenta $y_{h}=0$ corresponde a el espacio $A d S$; cuando es considerada, $y_{h}=0$ es impuesta en orden para obtener una correcta descripción del estado base y (246) puede ser usado para fijar el potencial químico, ver sección 5.5.
} 
Ya que fijaremos la posición del horizonte en esta forma, teniendo en mente que tenemos que considerar solo cantidades invariantes de escala.

En [83] y [33] las soluciones (241)-(242) con un horizonte y asintóticamente $A d S_{4}$ fueron estudiadas. Mas específicamente allí fueron encontradas soluciones con $K_{1}=K_{2}=K$ y las siguientes condiciones de borde: cerca del horizonte $y \rightarrow 1^{+}$,

$$
\begin{aligned}
f(y) & =f_{1}(y-1)+\mathcal{O}\left[(y-1)^{2}\right] \\
A(y) & =a_{0}+a_{1}(y-1)+\mathcal{O}\left[(y-1)^{2}\right] \\
c(y) & =c_{0}+c_{1}(y-1)+\mathcal{O}\left[(y-1)^{2}\right] \\
H(y) & =h_{0}+h_{1}(y-1)+\mathcal{O}\left[(y-1)^{2}\right] \\
K(y) & =k_{0}+k_{1}(y-1)+\mathcal{O}\left[(y-1)^{2}\right] \\
J(y) & =j_{1}(y-1)+\mathcal{O}\left[(y-1)^{2}\right]
\end{aligned}
$$

mientras que en el borde $y \rightarrow \infty$,

$$
\begin{aligned}
f(y) & =y^{2}+\frac{F_{1}}{y}+\cdots \\
A(y) & =1+\cdots \\
c(y) & =1+\cdots \\
H(y) & =1+\frac{H_{1}}{y^{3}}+\cdots \\
K(y) & =\frac{K_{1}}{y^{\kappa_{1}}}+\cdots \\
J(y) & =J_{0}+\frac{J_{1}}{y}+\cdots
\end{aligned}
$$

donde la consistencia con las e.o.m y la finitud de $K(y)$ fija $\kappa_{1}$ para ser

$$
\kappa_{1}\left(\kappa_{1}-1\right)=\hat{m}_{W}^{2} \quad \longrightarrow \quad \kappa_{1}=\frac{1}{2}+\sqrt{\frac{1}{4}+\hat{m}_{W}^{2}}
$$

para saber mas acerca de las b.c en el borde mandamos al lector al apéndice A. Adoptaremos las b.c. (247)-(248) en este trabajo excepto en la sección 5.5 donde las b.c sobre el horizonte tienden a ser modificadas.

La teoría definida en (237) es invariante bajo el grupo de grauge $S U(2)$; no obstante las b.c sobre el campo de Higgs $H(y) \stackrel{y \rightarrow \infty}{\longrightarrow} 1$, rompen esta invarianza al $U(1)$ generada por el $X_{0}$. Con respecto a este subgrupo de calibre la densidad de carga eléctrica de la solución es definida de la forma usual como,

$$
\left.\rho \equiv \frac{1}{V_{2}} \int_{\Re^{2}} * F\right|_{U(1)}=\left.\frac{1}{L^{2}} \frac{c(y)}{A(y)} y^{2} J^{\prime}(y)\right|_{y \rightarrow \infty}=-\frac{J_{1}}{L^{2}}
$$

como mostramos en la sección 5.4 para acoplamientos finitos $\left(\alpha, \hat{m}_{W}\right)$ una solución general de $(241)-(242)$ con las b.c (247)-(248) es determinada por $J_{0}$, la cual esta relacionada al potencial químico $U(1)$ por,

$$
\mu \equiv A_{0}^{0}(\infty)=\frac{J_{0}}{L}
$$

de (250) y (251) la expansión asintótica standard es

$$
A_{0}^{0}(y)=\mu-\frac{L \rho}{y}+\ldots
$$

A lo largo de este trabajo adoptaremos $\mu$ como nuestra escala. De (246) la temperatura invariante de escala adimensional es

$$
T \equiv \frac{T_{B H}}{\mu}=\frac{a_{0} f_{1}}{4 \pi J_{0}}
$$

donde $a_{0}$ y $f_{1}$ son definidas en (247). Una solución es determinada por los tres parámetros $\left(\alpha, \hat{m}_{W}, J_{0}\right)$, y entonces la temperatura (a través de los coeficientes $a_{0}, f_{1}$ ) resultan una función de ellos. ${ }^{14}$

En la solución analítica a las ecuaciónes (241)-(242) que preservan la simetría $U(1)_{X_{0}}$ los campos de materia toman la forma,

\footnotetext{
${ }^{14}$ En EYM donde el campo de Higgs no esta presente la temperatura es función de solo un parámetro libre de la teoría, $\alpha$, ver [34]
} 


$$
J(y)=J_{0}+\frac{J_{1}}{y}=J_{0}\left(1-\frac{1}{y}\right) \quad ; \quad K_{i}(y)=0 \quad ; \quad H(y)=1
$$

donde impusimos un comportamiento suave del campo de calibre en el horizonte dando la condición $J(1)=0$, ver la última línea en $(247)$, y entonces se encuentra $J_{1}=-J_{0}{ }^{15}$.

En lo que concierne a las funciónes de la métrica, ellas corresponden a un agujero negro AdS ReissnerNordström (AdS-RN),

$$
\begin{aligned}
A(y) & =c(y)=1 \\
f(y) & =y^{2}-\left(1+\frac{\alpha^{2} J_{0}^{2}}{2}\right) \frac{1}{y}+\frac{\alpha^{2} J_{0}^{2}}{2} \frac{1}{y^{2}} \\
& =\frac{y-1}{y^{2}}\left(3-\frac{\alpha^{2} J_{0}^{2}}{2}+\left(y^{2}+2 y+3\right)(y-1)\right)
\end{aligned}
$$

con temperatura,

$$
T=\frac{1}{4 \pi J_{0}}\left(3-\frac{\alpha^{2} J_{0}^{2}}{2}\right)
$$

El extremo (cero temperatura AdS-RN black hole) es definido por la relación $\alpha^{2} J_{0}^{2}=6$.

\subsection{Soluciones a $T>0$ : estado superconductor}

Cuando la parte magnética del campo de calibre es no trivial, i.e. $K_{i}(y) \neq 0$ para algún $i=1,2$, la solución no solo rompe la invarianza $U(1)_{X_{0}}$, sino que también rompe la invarianza bajo rotaciones en el plano $\left(x^{1}, x^{2}\right)$. De acuerdo al diccionario de $A d S / C F T$ este pelo es interpretado como un rompimiento espontáneo de una simetría global $U(1)$ en la teoría del borde (donde vive la QFT), cuya corriente toma un valor de expectación,

$$
\left\langle J_{i}^{a}(x)\right\rangle \sim K_{i} \delta_{i}^{a} \quad ; \quad i, a=1,2
$$

Dado que el parámetro de orden es dual a una componente del campo de calibre, es posible modelar un superconductor de onda $p[9]$. El estado normal del superconductor es descripto por una solución del tipo AdS-RN (254)-(255); tal solución es energéticamente favorecida hasta que cierta temperatura crítica $T_{c}$ es alcanzada; cuando $T<T_{c}$ la solución no simétrica con pelo, dando lugar a una fase superconductora. Cabe notar que con la b.c sobre el campo de Higgs estamos rompiendo explícitamente el grupo de calibre de $S U(2)$ a $U(1)_{X_{0}}$; esto da una masa para los bosones de calibre"W"

$$
m_{W} \equiv e H_{0}
$$

el problema es entonces el siguiente: podemos encontrar bajo esta condición una solución con $K_{i}(y) \neq 0$ que rompa espontáneamente el $U(1)_{X_{0}}$ ?. Esto ultimo es interpretado en el borde como un rompimiento de una fase de simetría global $U(1)$ de la QFT. Esto es parecido a lo que sucede con los superfluidos y superconductores con fotones débilmente acoplados. Se puede identificar a $T_{c}$ con la temperatura crítica de la transición de fase en la QFT.

Consideraremos dos casos:

- El caso isotrópico: $K(y) \equiv K_{1}(y)=K_{2}(y)$

Aunque ambos simetrías, la de calibre y la rotacional se rompen por una solución con pelo, una configuración (239) con $K_{1}(y)=K_{2}(y)$ preserva el subgrupo diagonal,

$\left(U(1)_{X_{0}} \times S O(2)_{\text {rot }}\right)_{\text {diag }}$, efecto que se manifiesta en (257) [24]. Esta configuración da lugar a un tensor de energía-momento isotrópico en el plano $x^{1}-x^{2}$; por lo tanto la componente de la métrica $c(y)$ debe ser una constante, incluso cuando el acoplamiento gravitacional es tomado en cuenta.

Este tipo de configuración fue primero estudiada en [83]-[33], usando métodos de relajación. Nosotros re-obtendremos estas soluciones usando shooting methods (métodos de disparo).

- El caso anisotrópico: $K(y) \equiv K_{1}(y) ; K_{2}(y)=0$

Como mencionamos arriba una configuración con $K_{1}=0$ preserva el grupo $U(1)_{X_{0}}$ y las rotaciones espaciales. Cuando $K_{1}(y)$ desarrolla un valor distinto de cero la simetría de calibre $U(1)_{X_{0}}$ se rompe,

\footnotetext{
${ }^{15}$ Cuando $y_{h}=0, J(y)=J_{0}$ es puro potencial químico y la solución de la métrica es un espacio $A d S$; este describe la fase no condensada cuando la temperatura es cero, ver sección 5
} 
y el condensado $K_{1}(y) X_{1} d x^{1}$ elije la dirección $x^{1}$ como especial. Luego si tenemos en cuenta el acoplamiento gravitacional, el sistema no puede soportar la condición $g_{11}=g_{22}$ [9]. Debido a este hecho $T_{x^{1} x^{1}} \neq T_{x^{2} x^{2}}$, la función $c(y)$ no puede ser una constante; en conclusión el sistema estará en una fase anisotrópica.

En ambos casos el valor de expectación del vacío en la teoría de campo $d=3$ del operador corriente, dual a la función $K$ asociada con el campo magnético en el lado gravitatorio, se sigue de la identificación, $\left\langle O_{K}\right\rangle \sim K_{1}$ con $K_{1}$ definido en $(248) ; K_{1}=K_{1}(T)$ puede ser tomado como el parámetro de orden que describe la transición de fase del sistema. Como discutimos para diferentes modelos [12]-[33] uno pude interpretar este resultado entendiendo que un condensado es formado arriba del horizonte del agujero negro debido a un balance entre las fuerzas gravitacionales y electrostáticas. Del comportamiento asintótico en (248) se obtiene la dimensión $\Delta\left[O_{K}\right]$ del operador $O_{K}[44]$

$$
\Delta\left[O_{K}\right]=1+\kappa_{1}=\frac{3}{2}+\frac{1}{2} \sqrt{1+4 \hat{m}_{W}^{2}}
$$

De las soluciones numéricas concluimos que a temperatura finita toma lugar una transición de fase debido a un rompimiento espontaneo de simetría y que el sistema condensa a una temperatura critica $T_{c}$, como se puede ver del comportamiento de $K_{1}(T)$ para $T \approx T_{c}$ en las figuras 27,28 y 29. Además si comparamos la energía libre correspondientes a ambas fases en las figuras 32 y 33, encontramos que la fase anisotrópica es favorecida, ver [40] [41] [42] para resultados relacionados.

\subsection{Soluciones numéricas}

Analizamos soluciones numéricamente (241)-(242) y encontramos soluciones que satisfacen las requeridas b.c (247)-(248) en una amplia región del espacio de parámetros, que conducen al diagrama de fase que se ve en la figura 25. Tales soluciones en el caso anisotrópico son mostradas en la figura 26.

Antes de presentar los resultados, pensamos que es mejor gastar unas pocas palabras sobre el método usado para encontrar tales soluciones. Como discutimos en el apéndice A1, después de fijar alguna normalización y preguntarnos por la finitud de la solución cerca del borde, la solución es determinada por seis constantes $\left(F_{1}, C_{1}, J_{0}, J_{1}, K_{1}, H_{1}\right)$. De todas maneras las b.c sobre el horizonte impone cinco condiciones. Las primeras dos viene de la definición del horizonte y la regularidad del campo de calibre,

$$
f(1)=0 \quad ; \quad J(1)=0
$$

Estas esencialmente fijan la masa y la densidad de carga $\left(\sim J_{1}\right)$ del agujero negro. Las otras tres condiciones fijan $\left(C_{1}, K_{1}, H_{1}\right)$ y son obtenidas de un análisis del comportamiento (singular) de las e.o.m cerca del horizonte,

$$
\begin{aligned}
c^{\prime}(1) & =\alpha^{2} \hat{m}_{W}{ }^{2} \frac{c(1)}{f^{\prime}(1)}\left(-\frac{K_{1}(1)^{2}}{c(1)^{2}}+K_{2}(1)^{2}\right) H(1)^{2} \\
K_{1}^{\prime}(1) & =\frac{K_{1}(1)}{f^{\prime}(1)}\left(K_{2}(1)^{2}+\hat{m}_{W}{ }^{2} H(1)^{2}\right) \\
H^{\prime}(1) & =\frac{H(1)}{f^{\prime}(1)}\left(\frac{K_{1}(1)^{2}}{c(1)^{2}}+K_{2}(1)^{2}\right)
\end{aligned}
$$

Por lo tanto el único parámetro adicional que determina la solución es $J_{0}$, i.e el potencial químico (251). En la practica integramos el sistema desde el horizonte, donde de acuerdo a (260)-(261) los parámetros libres son,

$$
J^{\prime}(1)=j_{1} \quad ; \quad K(1)=k_{0} \quad ; \quad H(1)=h_{0} \quad ; \quad A(1)=a_{0} \quad ; \quad c(1)=c_{0}
$$

como fueron definidos en (247). Estos parámetros son elegidos de una forma tal que las soluciones le peguen a las condiciones sobre el borde (248),

$$
A(\infty)=c(\infty)=H(\infty)=1 \quad ; \quad K(\infty)=0 \quad ; \quad J(\infty)=J_{0}
$$



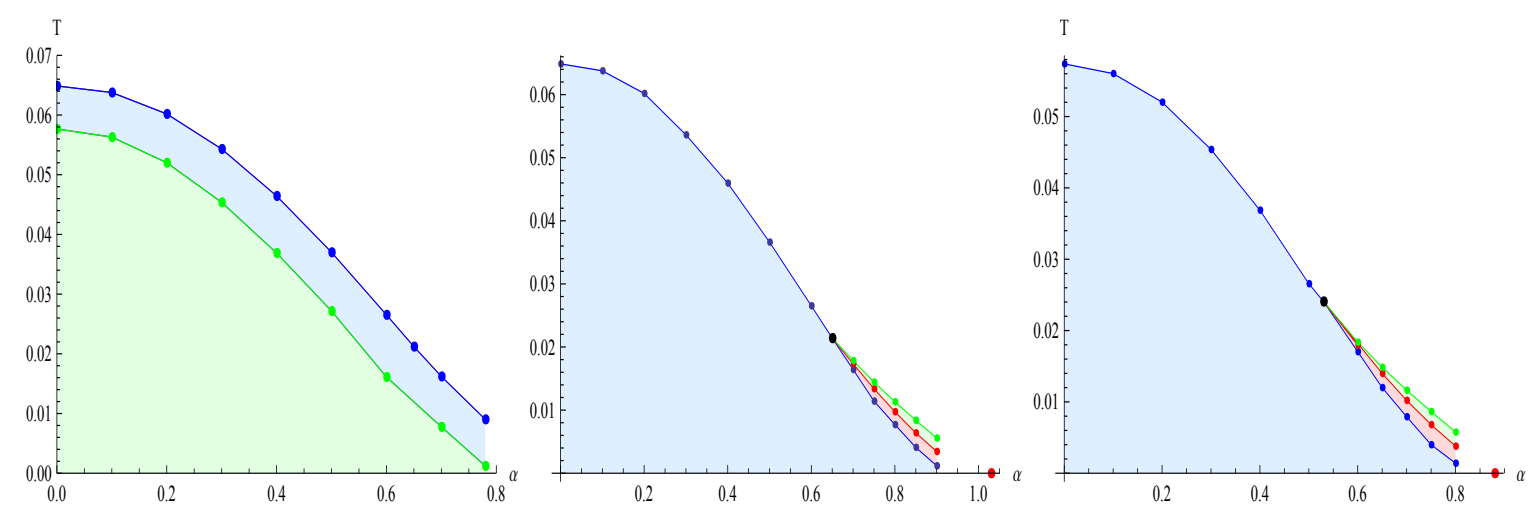

Figura 25: Diagramas de fases en el caso isotropico (izquierda) para $\hat{m}_{W}=0.1$ (azul), $\hat{m}_{W}=0.4$ (verde), y en el caso anisotrópico, para $\hat{m}_{W}=0.1$ (centro) y $\hat{m}_{W}=0.4$ (derecha).

La figura 25 muestra el diagrama de fases en el plano $\alpha-T$, para dos diferentes valores de $\hat{m}_{W}$. En las regiónes blancas solo la fase normal o no condensada esta presente. En el caso isotrópico el sistema experimenta una transición de segundo orden a lo largo de las curvas azules $\left(\hat{m}_{W}=0.1\right)$ y verdes $\left(\hat{m}_{W}=0.4\right)$. En el caso anisotrópico, en la regiónes azules y rojas la fase condensada es una fase termodinámica preferida. La línea azul hasta el punto negro indica una línea crítica de transiciones de segundo orden. El punto negro señala el acoplamiento $\alpha_{t c}$ más allá del cual la transición se convierte de primer orden a lo largo de la línea roja, mientras que las líneas azules y verdes que continúan después del triple punto crítico representan líneas espinodales. La curva roja crítica, de las transiciones de primer orden terminan en el punto rojo a $T=0$, las cuales representan una transición de fase cuántica, señalando un acoplamiento crítico $\tilde{\alpha}$ arriba del cual la fase condensada deja de existir, ver sección 5.5. Comparando ambos gráficos podemos ver que ambos $\alpha_{t c}$ y $\tilde{\alpha}$ decrecen con el incremento de $\hat{m}_{W}$. Diagramas de fase similares son obtenidos en las referencias [45] en ausencia de campo de Higgs.

En la figura 26 los campos son mostrados como función de la coordenada $y$, a un valor fijo de $J_{0}$ y $\hat{m}_{W}$ y para diferentes $\alpha$ 's. Para $\alpha_{c} \approx 0.8825$ un segundo horizonte aparece, como se aprecia de las curvas correspondientes a $f(y) / y^{2}$. La fase no condensada y condensada están separadas por una curva sobre la cual la formación de un segundo horizonte toma lugar para una dada temperatura crítica determinada por la masa del bosón de calibre $\hat{m}_{W}$ y $J_{0}$.

En el caso isotrópico la curva es mostrada en la figura 25 para dos diferentes valores de $\hat{m}_{W}$ (líneas azules y verdes) y coincide con la curva crítica sobre la cual la transición de fase toma lugar. Por otro lado, en el caso anisotrópico la curva coincide con la curva crítica (línea azul en la figura 25) hasta el triple punto crítico $\alpha_{t c}$, y esta continua a través de la curva espinodal verde.

En la figura 27 el parámetro de orden $K_{1}$ es mostrado como función de la temperatura para diferentes valores de $\hat{m}_{W}$, para un acoplamiento gravitacional fijo $\alpha=0.7$. En este caso la transición es de segundo orden independientemente de $\alpha$, en acuerdo con [24].

Las figuras 28 y 29 muestran el parámetro de orden $K_{1}$ como función de $T$ en el caso anisotrópico desde dos perspectivas: para un valor fijo de $\hat{m}_{W}=0.4$ y variando $\alpha$ de la figura 28 y luego a un valor fijo de $\alpha=0.7$ y variando $\hat{m}_{W}$ en la figura 29 . De la figura 28 se ve que para $\alpha_{t c} \approx 0.53 K_{1}$ se convierte en multievaluada, hecho que señala el pasaje de una transición de segundo a una de primer orden como se corrobora del computo de la energía libre en la siguiente sección. Este fenómeno ha sido visto recientemente en superfluidos de onda $-p$ estudiando el papel del acoplamiento gravitacional en las transiciones de fase [34] (para resultados experimentales sobre transiciones de fase de primer orden en superconductores, ver [37][38][39]). Comparando las figuras 27 y 29 es observado que la temperatura a la cual el parámetro de orden se convierte en cero es el mismo en ambos casos, y que la temperatura crítica decrece cuando $\hat{m}_{W}$ se incrementa, esto puede ser interpretado como que la presencia del campo de Higgs dificulta la condensación. Además hemos chequeado cerca de la temperatura crítica $T_{c}$ y para acoplamientos gravitacionales pequeños $\alpha<\alpha_{t c}$, que $K_{1}$ se comporta como $\left(T_{c}-T\right)^{\frac{1}{2}}$, indicando una transición de fase de segundo orden con un exponente de campo medio de $\left(T_{c}-T\right)^{\frac{1}{2}}$, como usualmente sucede en descripciones holográficas de sistemas críticos en el límite de un gran número de grados de libertad. 

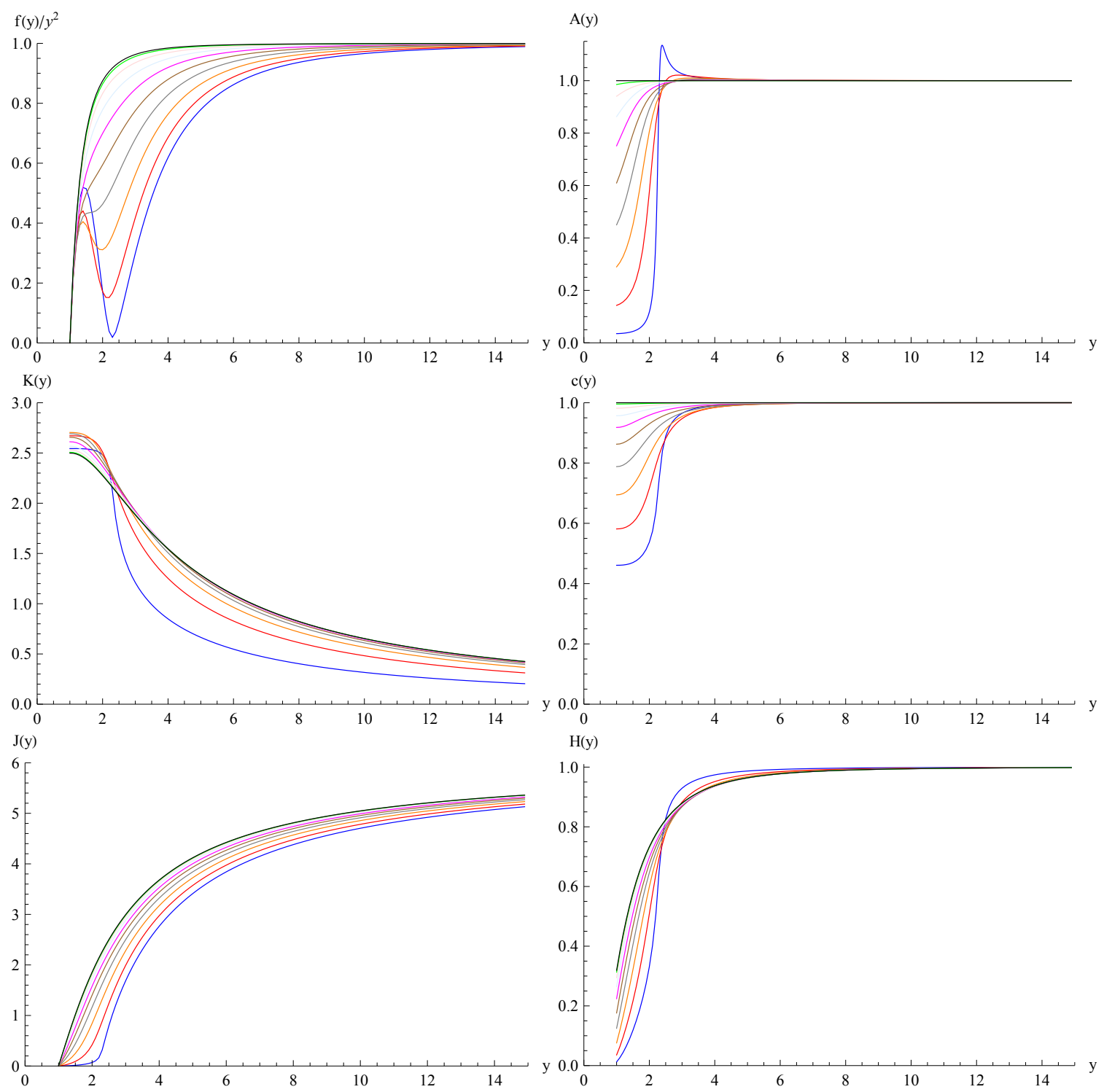

Figura 26: soluciones para los campos $(f(y), A(y), c(y), K(y), J(y), H(y))$ con b.c (247)-(248) en el caso anisotrópico. Las curvas corresponden a $J_{0}=6, \hat{m}_{W}=0.4$ con diferentes valores de $\alpha=0.0$ (negra), 0.1 (verde), 0.2 (roja suave), 0.3 (azul suave), 0.4 (fucsia), 0.5 (marrón), 0.6 (gray), 0.7 (naranja), 0.8 (roja), 0.88 (azul). Se puede apreciar de las curvas correspondientes a $f(y) / y^{2}$ la formación de un segundo horizonte en $\alpha_{c} \approx 0.8825$. Las soluciones análogas para el caso isotrópico pueden ser encontradas en [33]. 


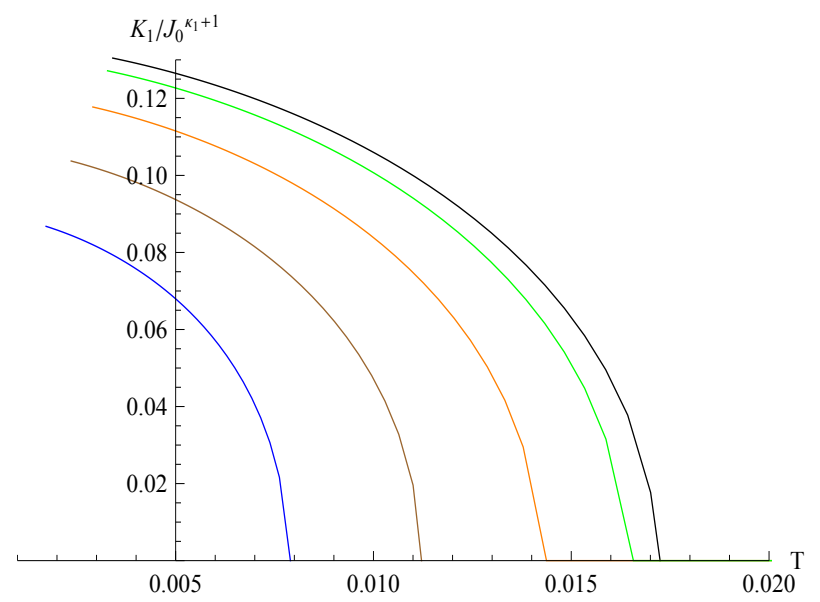

Figura 27: El parámetro de orden $K_{1} / J_{0}{ }^{1+\kappa_{1}}=\left\langle\hat{O}_{K_{1}}\right\rangle / J_{0}{ }^{1+\kappa_{1}}$ es mostrado en el caso isotrópico a un valor fijo de $\alpha=0.7$, para diferentes valores de $\hat{m}_{W}=0.01$ (negra), 0.1 (verde), 0.2 (naranja), 0.3 (marrón), 0.4 (azul) que corresponden a las temperaturas críticas $T_{c}=0.017056,0.016371,0.014174,0.011215,0.00791$ respectivamente. Cerca de la temperatura crítica el parámetro de orden se comporta como $\left(T_{c}-T\right)^{1 / 2}$.

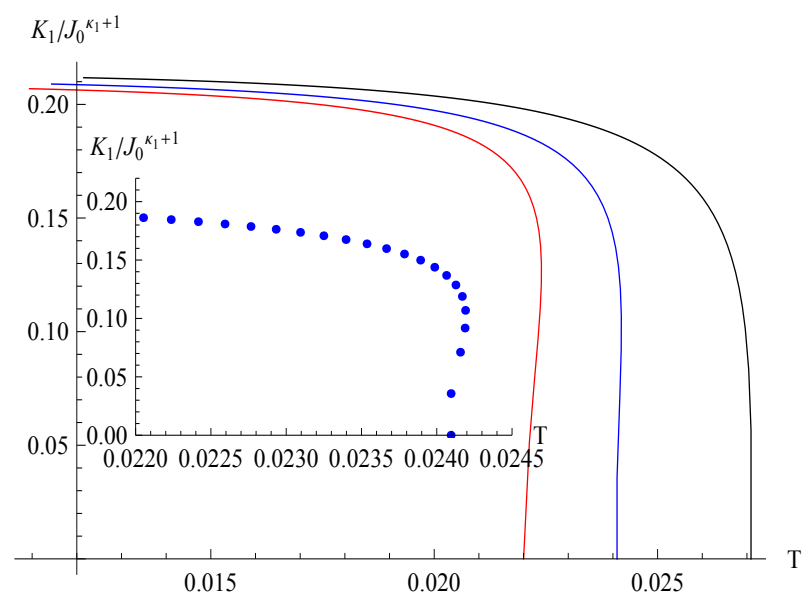

Figura 28: El parámetro de orden $K_{1} / J_{0}{ }^{1+\kappa_{1}}=\left\langle\hat{O}_{K_{1}}\right\rangle / J_{0}{ }^{1+\kappa_{1}}$ es mostrado en el caso anisotrópico para un valor fijo de $\hat{m}_{W}=0.4$, para diferentes valores de $\alpha=0.50$ (negra), 0.53 (azul), 0.55 (roja). En el recuadro es mostrada la multievaluación del parámetro de orden para $\alpha_{t c} \approx 0.53$. 


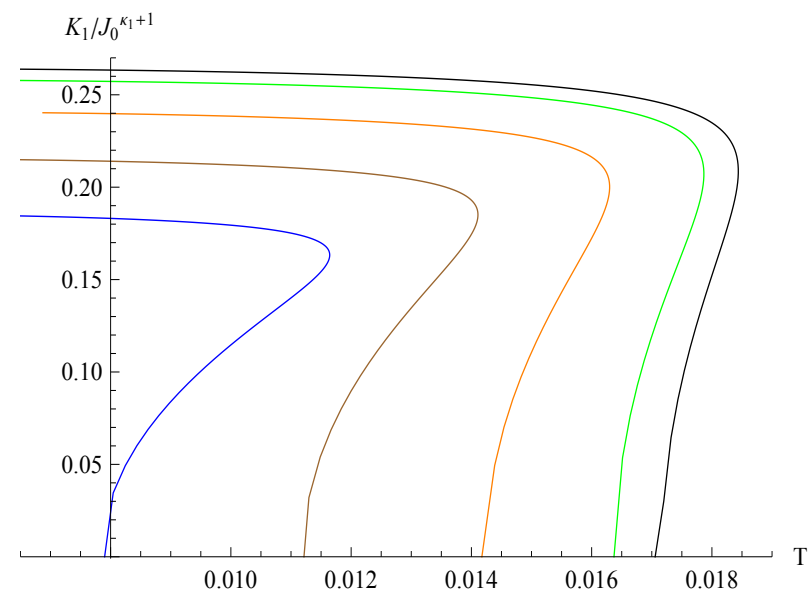

Figura 29: El párametro de orden $K_{1} / J_{0}{ }^{1+\kappa_{1}}=\left\langle\hat{O}_{K_{1}}\right\rangle / J_{0}{ }^{1+\kappa_{1}}$ es mostrado en el caso anisotrópico a un valor fijo de $\alpha=0.7$, mostrando la transición de fase a diferentes valores de $\hat{m}_{W}=0.01$ (negro), 0.1 (verde), 0.2 (naranja), 0.3 (marrón), 0.4 (azul).

\subsubsection{La energía libre}

De acuerdo a la correspondencia AdS/CFT, la energía libre de la QFT es dada por,

$$
F \equiv T S_{\text {eucl }}=\int_{y_{h}}^{\infty} d y \int d \vec{x}^{2} L_{\text {eucl }}
$$

de (237) y usando las e.o.m la contribución de los campos de materia-gravedad a la densidad de energía libre puede ser escrita como,

$$
\begin{aligned}
f^{(\text {bulk })} & =\frac{1}{2 e^{2} L^{3}} \int_{1}^{y_{\infty}} d y A(y) c(y) y^{2}\left(\frac{6}{\alpha^{2}}-\frac{\lambda_{0}}{2}\left(H(y)^{2}-1\right)^{2}\right. \\
& +\frac{f(y)}{y^{2}}\left(\frac{K_{1}^{\prime}(y)^{2}}{c(y)^{2}}+K_{2}^{\prime}(y)^{2}\right)+\frac{K_{1}(y)^{2} K_{2}(y)^{2}}{y^{4} c(y)^{2}} \\
& \left.-\frac{J^{\prime}(y)^{2}}{A(y)^{2}}-\frac{J(y)^{2}}{y^{2} f(y) A(y)^{2}}\left(\frac{K_{1}(y)^{2}}{c(y)^{2}}+K_{2}(y)^{2}\right)\right)
\end{aligned}
$$

La contribución del termino de Gibbons-Hawking es,

$$
f^{(G H)}=\left.\frac{1}{2 e^{2} L^{3}}\left(-\frac{2}{\alpha^{2}}\right) f(y)^{\frac{1}{2}}\left(y^{2} f(y)^{\frac{1}{2}} A(y) c(y)\right)^{\prime}\right|_{y_{\infty}}
$$

Aquí hemos introducido $y_{\infty}$ para regularizar las expresiones ya que ellas presentan términos divergentes. Para este fin hemos introducido un contratérmino a la acción [46] [47],

$$
S^{(c t)}=\frac{1}{2 \kappa^{2}} \int_{\partial \mathcal{M}} d^{3} x \sqrt{|h|} \frac{-2}{L}
$$

el cual da lugar a la siguiente contribución a la densidad de energía libre,

$$
f^{(c t)}=\left.\frac{1}{2 e^{2} L^{3}} \frac{4}{\alpha^{2}}\left(f(y)^{1 / 2} A(y) c(y) y^{2}\right)\right|_{y_{\infty}}
$$

La densidad total de energía libre del sistema $f$ es dada por,

$$
f \equiv \lim _{y \infty \rightarrow \infty}\left(f^{(b u l k)}+f^{(G H)}+f^{(c t)}\right)
$$

Remarcamos que en orden para analizar los resultados, la forma correcta es trabajar con la densidad de energía libre invariante de escala,

$$
\hat{f} \equiv \frac{\kappa^{2}}{L^{2} \mu^{3}} f
$$


Las figuras 30 y 31 muestran la evolución de la densidad de energía libre (270) con la masa del bosón de calibre para dos diferentes valores de $\alpha$, en los casos isotrópicos y anisotrópicos respectivamente. La figura 30 muestra la continuidad de $\hat{f}$ a la temperatura crítica (donde la densidad de energía libre de la fase no condensada se intersecta con la fase de la curva condensada) para ambos valores de $\alpha$, para cualquier $\hat{m}_{W}$, hecho que indica el carácter de segundo orden de la transición de fase como el comportamiento de $K_{1}$ en la figura 27 lo sugiere. En la figura 31 en efecto se observa la discontinuidad en la primera derivada de la densidad de energía libre a la temperatura crítica para $\alpha=0.7>\alpha_{t c}$ para cualquier valor $\hat{m}_{W}$, señala una transición de fase de primer orden. En ambos casos la temperatura crítica decrece con el crecimiento de $\hat{m}_{W}$, en acuerdo con el análisis del comportamiento del parámetro de orden de arriba.

En las figuras 32 y 33 las densidad de energía libre de las fases isotrópicas y anisotrópicas son comparadas para dos valores del acoplamiento gravitacional, $\alpha<\alpha_{t c}$ (figura 32) y $\alpha>\alpha_{t c}$ (figura 33). De ellas uno puede ver que la densidad de energía de la fase anisotrópica, sin importar el valor que tome $\alpha$ i.e si estamos en la fase de primer o de segundo orden, es mas baja que la densidad de energía libre de la fase isotrópica. Esto implica, que la fase anisotrópica es siempre energéticamente favorecida sobre la fase isotrópica.

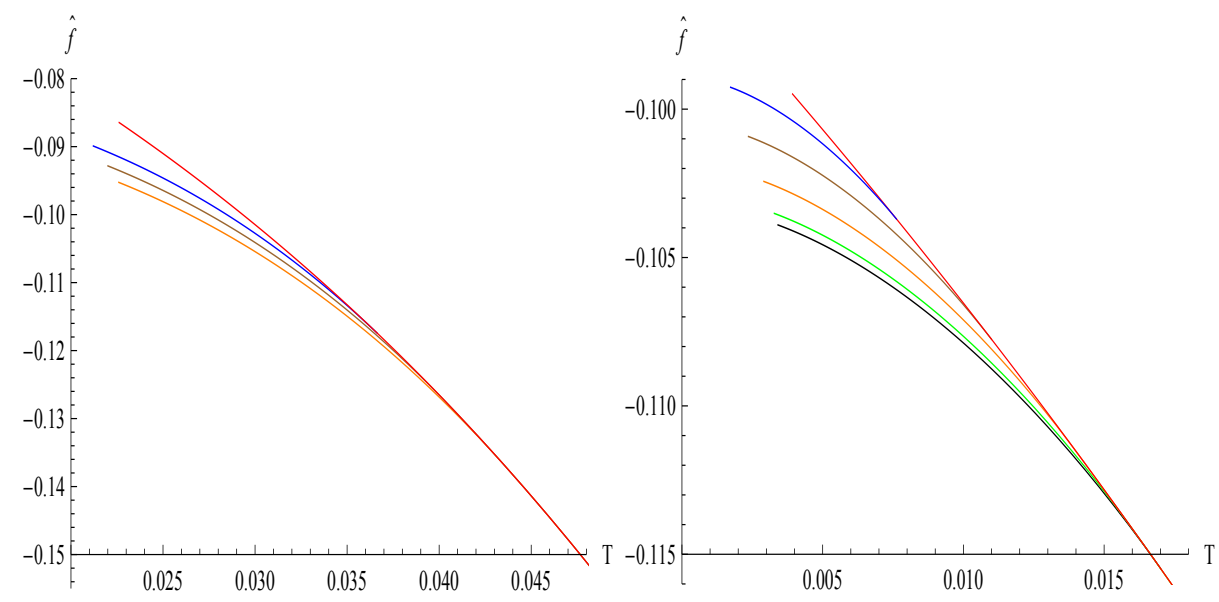

Figura 30: La densidad de energía libre $\hat{f}$ se muestra como función de la temperatura en el caso isotrópico para dos valores del acoplamiento gravitacional $\alpha=0.4$ (izquierda) y $\alpha=0.7$ (derecha), a diferentes valores de $\hat{m}_{W}=0.01$ (negra), 0.1 (verde), 0.2 (naranja), 0.3 (marrón), 0.4 (azul). La curva roja representa la densidad de energía libre de la fase no condensada.
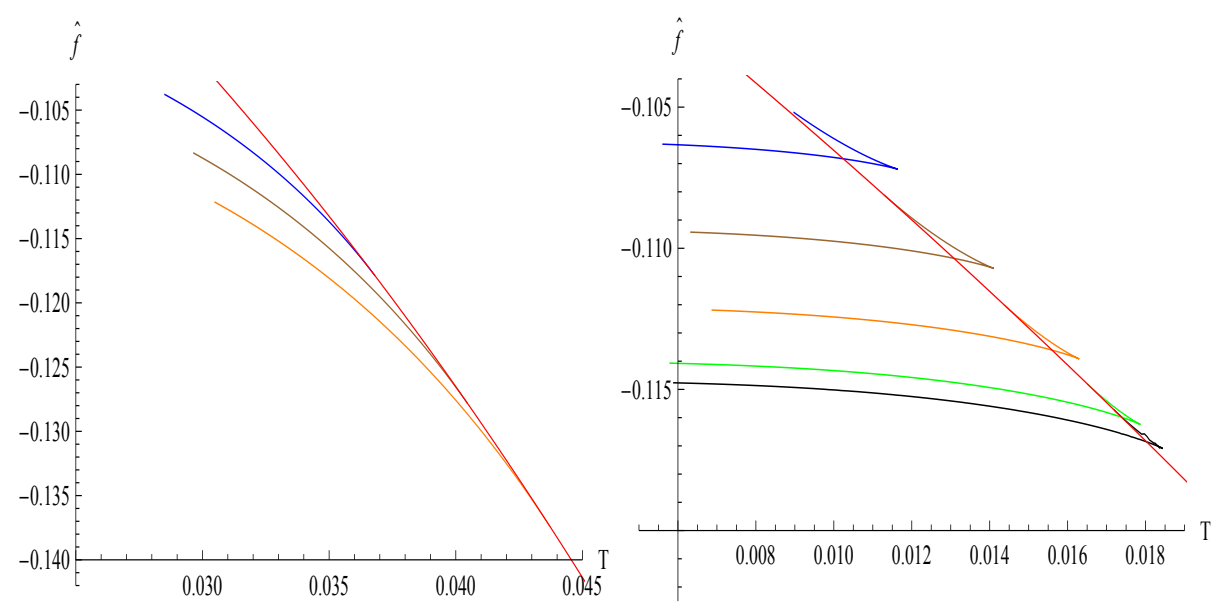

Figura 31: La densidad de energía libre $\hat{f}$ se muestra como función de la temperatura en el caso anisotrópico para dos valores del acoplamiento gravitacional $\alpha=0.4$ (izquierda) y $\alpha=0.7$ (derecha), a diferentes valores de $\hat{m}_{W}=0.01$ (negra), 0.1 (verde), 0.2 (naranja), 0.3 (marrón), 0.4 (azul). La curva roja representa la densidad de energía libre de la fase no condensada. 

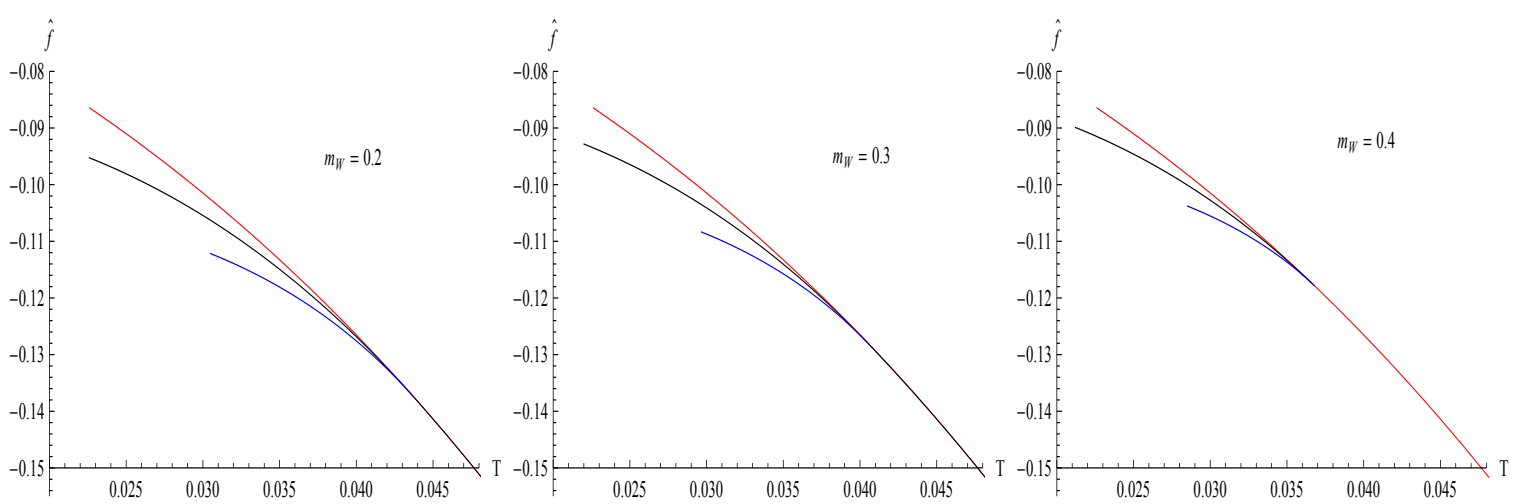

Figura 32: Las densidades de energía libre para los casos isotrópico (negra) y anisotrópico (azul) son mostradas como función de la temperatura para diferentes valores de $\hat{m}_{W}$, a un valor fijo de $\alpha=0.4$. La curva roja representa la densidad de energía libre de la fase no condensada.

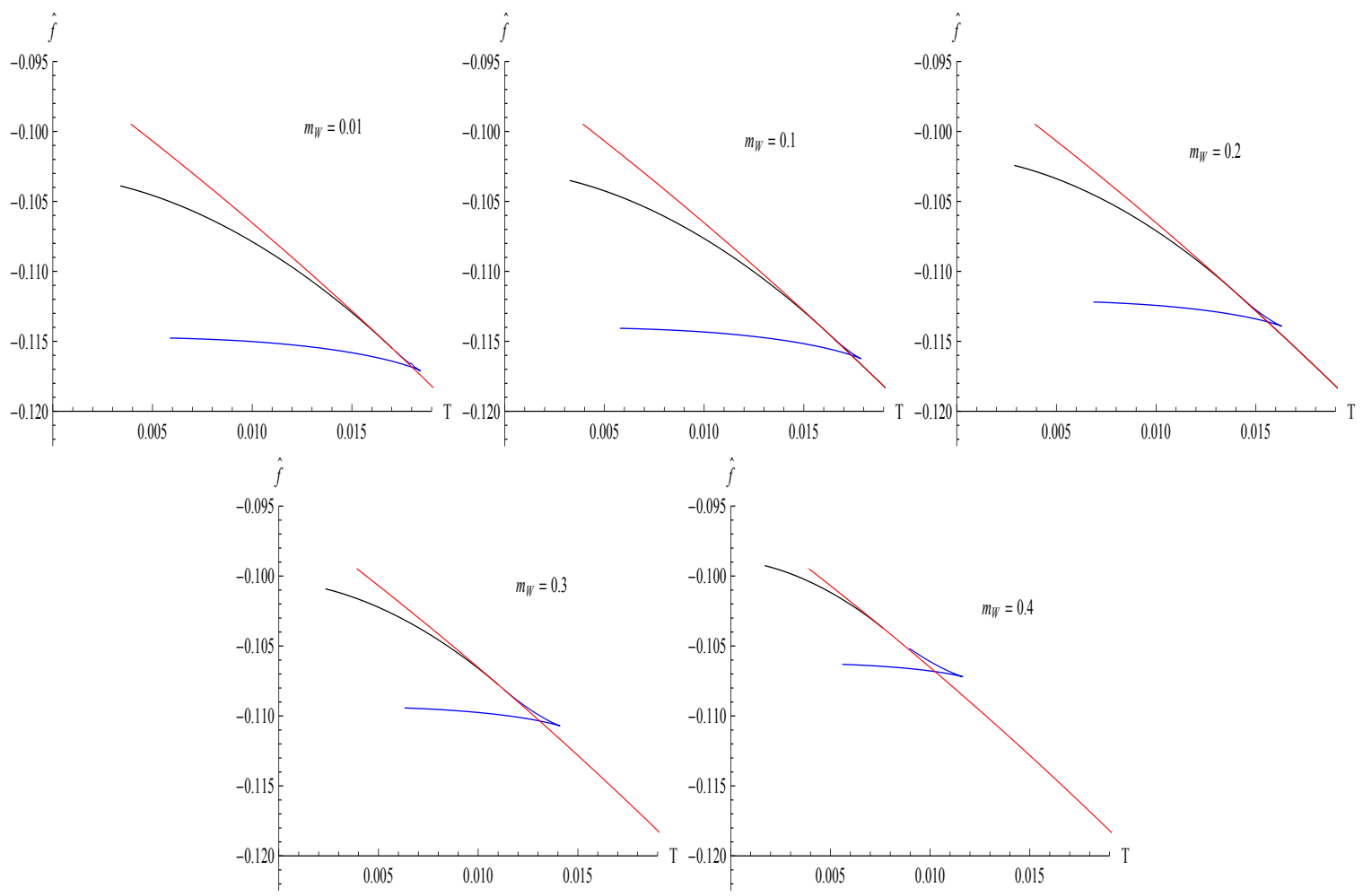

Figura 33: Las densidades de energía libre para los casos isotrópicos (negra) y anisotrópicos (azul) son mostrados como función de la temperatura para diferentes valores de $\hat{m}_{W}$, a un valor fijo de $\alpha=0.7$. La curva roja representa la densidad de energía libre de la fase no condensada.

\subsection{Soluciones a $T=0$}

En esta sección analizaremos el problema de transiciones de fase cuánticas en tres dimensiones en un superconductor de onda -p (en el caso anisotrópico), i.e transiciones a $T=0$, que ha nuestro conocimiento no ha sido considerado antes en la literatura (ver no obstante [48] [49] [50] [51] para estudios relacionados en otros modelos).

Es conocido que cuando un agujero negro cargado AdS es llevado hacia el límite de temperatura cero este se convierte en un agujero negro extremo, pero su entropía es diferente de cero y entonces tal sistema no puede describir el estado base del superconductor que queremos modelar holográficamente. Para alcanzar tal objetivo el radio del agujero negro necesita volverse nulo, para estar en acuerdo con la tercera ley de la termodinámica y realmente describir el estado fundamental cuántico del superconductor. Entonces debemos 
imponer que $y_{h}=0$, , con lo cual la coordenada $y \in[0, \infty)$. Una muy importante cosa desde un punto de vista técnico es que mientras que el comportamiento asintótico de los campos es como en (248), las expansiones cerca del horizonte cambian drásticamente con respecto al caso $T>0$. A primer orden el comportamiento no analítico de los campos para $y \rightarrow 0^{+}$es,

$$
\begin{aligned}
& f(y)= y^{2}-\alpha^{2} \frac{\tilde{k}_{0} \tilde{j}_{0}^{2}}{2 \tilde{c}_{0} \tilde{a}_{0}^{2}} \frac{e^{-\frac{2 \tilde{k}_{0}}{\tilde{c}_{0} y}}}{y}+\ldots \\
& A(y)= \tilde{a}_{0}\left(1+\alpha^{2} \frac{\tilde{k}_{0} \tilde{j}_{0}^{2}}{\tilde{c}_{0} \tilde{a}_{0}^{2}} \frac{e^{-\frac{2 \tilde{k}_{0}}{\tilde{c}_{0} y}}}{y^{3}}+\ldots\right) \\
& c(y)= \tilde{c}_{0}\left(1+\alpha^{2} \frac{\tilde{j}_{0}^{2}}{\tilde{a}_{0}^{2}} \frac{e^{-\frac{2 \tilde{k}_{0}}{\tilde{c}_{0} y}}}{y^{2}}+\ldots\right) \\
& K(y)= \tilde{k}_{0}\left(1-\frac{\tilde{c}_{0}^{2} \tilde{j}_{0}^{2}}{4 \tilde{a}_{0}^{2} \tilde{k}_{0}^{2}} e^{-\frac{2 \tilde{k}_{0}}{\tilde{c}_{0} y}}+\ldots\right) \\
& J(y)= \tilde{j}_{0} e^{-\frac{\tilde{k}_{0}}{\tilde{c}_{0} y}}+\ldots \\
& H(y)=\tilde{h}_{0} e^{-\frac{\tilde{k}_{0}}{\tilde{c}_{0} y}}+\ldots
\end{aligned}
$$

Las constantes independientes son $\tilde{k}_{0}, \tilde{j}_{0}, \tilde{h}_{0}, \tilde{a}_{0}, \tilde{c}_{0}$. Tales constantes son elegidas de la misma forma como en el caso de $T>0$, ver (263). De (271) se sigue que cerca del horizonte la solución es otro espacio $A d S_{4}$. La solución que describe el estado base cuántico del superconductor en la fase condensada son por lo tanto paredes de dominio (domain walls) que interpolan la solución entre espacios $A d S_{4}$ con el mismo radio $L$ pero con diferentes velocidades de la luz en ambas direcciones, en virtud del hecho que $\tilde{a}_{0} \neq 1$ y $\tilde{c}_{0} \neq 1$. Mas explícitamente,

$$
\frac{v_{1}^{U V}}{v_{1}^{I R}}=\frac{y_{h}}{y_{\infty}} \frac{c\left(y_{h}\right)}{c\left(y_{\infty}\right)} \sqrt{\frac{f\left(y_{\infty}\right)}{f\left(y_{h}\right)}} \frac{A\left(y_{\infty}\right)}{A\left(y_{h}\right)}=\frac{\tilde{c}_{0}}{\tilde{a}_{0}} \quad ; \quad \frac{v_{2}^{U V}}{v_{2}^{I R}}=\frac{y_{h}}{y_{\infty}} \sqrt{\frac{f\left(y_{\infty}\right)}{f\left(y_{h}\right)}} \frac{A\left(y_{\infty}\right)}{A\left(y_{h}\right)}=\frac{1}{\tilde{a}_{0}}
$$

Por otro lado, la fase no condensada es descripta estrictamente por el espacio $A d S_{4}$ y $J(y)=J_{0}$, la cual reemplaza la solución AdS-RN (254)-(255). También hemos encontrado que arriba de cierto valor de $\tilde{\alpha}$ la solución que representa la fase condensada desaparece y la única solución que existe es el espacio AdS. Este resultado puede ser seguido del análisis de [49] (ver también [40]). A muy bajas temperaturas la fase normal es representada casi por la solución de un agujero negro Reissner-Nördstrom (254-256) con $J_{0}^{2}=\frac{6}{\alpha^{2}}$, cuya geometría cerca del horizonte es $A d S_{2} \times \Re^{2}$. Si perturbamos esta solución con un campo de calibre no nulo $K_{1}(y)=K(y)$, de la primera ecuación en (242) su ecuación líneal en esta geometría resulta ser,

$$
0=\left(\rho^{2} \partial_{\rho}^{2}+2 \rho \partial_{\rho}-\hat{m}_{e f f}^{2}\right) K(y)
$$

donde $\rho \equiv y-1$, la cual es solo la ecuación de onda para $A d S_{2}$ con una masa efectiva,

$$
\hat{m}_{e f f}^{2}=\frac{1}{6}\left(\hat{m}_{W}^{2}-\frac{1}{\alpha^{2}}\right)
$$

con lo cual, la inestabilidad para formar un pelo vectorial $S U(2)$ a bajas temperaturas, sucede para campos escalares por debajo del enlace de BF para $A d S_{2}, \hat{m}_{B F}^{2}=-\frac{1}{4}$. Esto es, cuando $\hat{m}_{e f f}{ }^{2}<\hat{m}_{B F}^{2}$ uno puede esperar que el vacío del espacio $A d S$ se vuelva inestable y el sistema prefiera estar en la fase descripta por la solución superconductora del agujero negro con pelo no abeliano. Entonces obtenemos una adecuada condición para la inestabilidad,

$$
\alpha^{2}<\tilde{\alpha}_{\text {guess }}^{2} \equiv \frac{1}{\frac{3}{2}+\hat{m}_{W}^{2}}
$$

La figura 34 muestra los campos para diferentes valores del acoplamiento gravitacional ${ }^{16}$. En el ejemplo mostrado $\left(\hat{m}_{W}=0.4\right)$ obtenemos $\left.\tilde{\alpha} \approx 0.8825 \approx \alpha_{c}\right|_{T>0}$, ver figuras 26 y 34 .

\footnotetext{
${ }^{16}$ Usaremos la simetría de escala (246) para fijar $J_{0}=1$.
} 

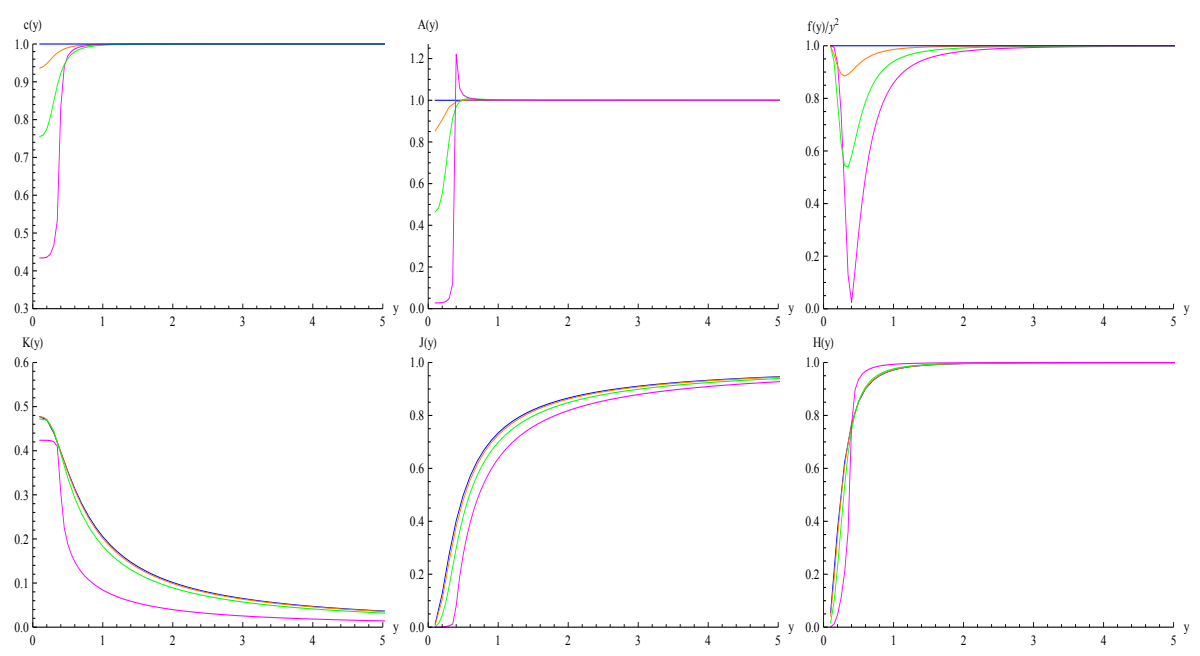

Figura 34: Mostramos para $T=0$ las soluciones $f(y) / y^{2}, A(y), c(y), H(y), K(y)$ and $J(y)$ como función de la coordenada $y$ desde el horizonte, ahora localizado en $y=0$, hacia el borde, para $\hat{m}_{W}=0.4$ y diferentes valores de $\alpha=0.0$ (azul), 0.3 (naranja), 0.6 (verde), 0.8 (fucsia). Se puede apreciar de las curvas correspondientes a $f(y) / y^{2}$ la formación de un segundo horizonte cuando $\alpha$ se aproxima a $\left.\tilde{\alpha} \approx \alpha_{c}\right|_{T>0} \approx 0.8825$; para valores mas grandes que $\alpha$ la solución asimétrica deja de existir.

Encontramos las soluciones para diferentes valores del parámetro $\hat{m}_{W}$, en la tabla 1 los correspondientes acoplamientos críticos son mostrados. Es observado que $\tilde{\alpha}$ decrece cuando se incrementa $\hat{m}_{W}$ y que $\tilde{\alpha}_{\text {guess }}<$ $\tilde{\alpha}$, esto es consistente con en análisis de inestabilidad realizado arriba.

Por otro lado, en la figura 35 es mostrado el parámetro de orden como función del acoplamiento $\alpha$. Hemos verificado que el comportamiento cerca del acoplamiento crítico es del tipo,

$$
K_{1}(\alpha) \sim(\tilde{\alpha}-\alpha)^{\frac{1}{2}}
$$

consistente con la existencia de una transición de segundo orden en la teoría de campo medio, en el límite de un gran número de grados de libertad.

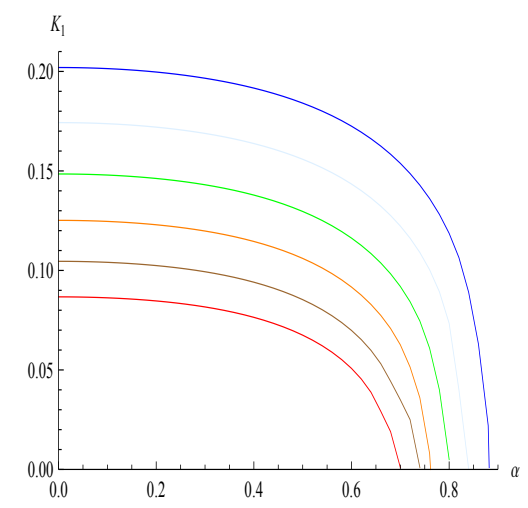

Figura 35: El parámetro de orden $K_{1}$ es mostrado como función de $\alpha$ a $T=0$, para diferentes valores de $\hat{m}_{W}=0.5$ (azul), 0.6 (azul claro), 0.7 (verde), 0.8 (naranja), 0.9 (marrón), 1.0 (rojo). Los valores para los cuales $K_{1}=0$ define los acoplamientos críticos $\tilde{\alpha}$.

\subsection{Análisis para $\lambda \neq 0$}

En esta sección estudiaremos el efecto de un potencial de Higgs no nulo como es introducido en (237), especificado por la escala del vev del Higgs $H_{0}$ y la intensidad $\lambda$. Por simplicidad trabajaremos en ausencia de acoplamiento gravitacional $\alpha$, aunque la nueva idea no depende de este hecho.

En las convenciones del apéndice A2, las e.o.m (242) en el caso anisotrópico se reducen a, 


\begin{tabular}{|c|c|}
\hline$\hat{m}_{W}$ & $\tilde{\alpha}$ \\
\hline 0.1 & 1.03 \\
\hline 0.2 & 0.97 \\
\hline 0.3 & 0.94 \\
\hline 0.4 & 0.88 \\
\hline 0.5 & 0.86 \\
\hline 0.6 & 0.84 \\
\hline 0.7 & 0.80 \\
\hline 0.8 & 0.76 \\
\hline 0.9 & 0.74 \\
\hline 1.0 & 0.70 \\
\hline
\end{tabular}

Tabla 1: Acoplamientos gravitacionales críticos $\tilde{\alpha}$ para diferentes valores de $\hat{m}_{W}$ y $T=0$.

$$
\begin{aligned}
\left(f(u) K^{\prime}(u)\right)^{\prime} & =\left(\hat{m}_{W}{ }^{2} \frac{H(u)^{2}}{u^{2}}-\frac{J(u)^{2}}{f(u)}\right) K(u) \\
u^{2}\left(\frac{f(u)}{u^{2}} H^{\prime}(u)\right)^{\prime} & =\left(K(u)^{2}+\frac{\lambda_{0}}{\hat{m}_{W}} \frac{H(u)^{2}-1}{u^{2}}\right) H(u) \\
J^{\prime \prime}(u) & =\frac{K(u)^{2}}{f(u)} J(u)
\end{aligned}
$$

donde $f(u)=1-u^{3}$.

La existencia de un $\lambda$ no nulo no modifica el comportamiento de $K(y)$ y $J(y)$ sobre el borde, siguen permaneciendo como en (248), pero en el caso del Higgs tenemos,

$$
H(u)=1+H_{-} u^{\Delta_{-}}+\cdots+H_{+} u^{\Delta_{+}}+\ldots
$$

donde, para un valor general de $\lambda_{0}$ y $\hat{m}_{W}$

$$
\Delta_{ \pm}=\frac{3}{2} \pm \sqrt{\frac{9}{4}+2 \frac{\lambda_{0}}{\hat{m}_{W}{ }^{2}}}
$$

Un hecho bien conocido es que exigiendo un valor real de $\Delta_{ \pm}$esto necesariamente implica que el enlace BF $\lambda_{0} \geq-\frac{9}{8} \hat{m}_{W}{ }^{2}$. Cuando estamos en la ventana $-\frac{9}{8} \leq \frac{\lambda_{0}}{\hat{m}_{W}{ }^{2}} \leq-\frac{3}{4}$ ambos modos son normalizables y conducen a una cuantización consistente y podemos ignorar $H_{-}=0$ o $H_{+}=0$. Si $\lambda_{0}>-\frac{3}{4} \hat{m}_{W}^{2}$, la condición $H_{-}=0$ debe ser impuesta [53]. Consideraremos por definición el caso $\lambda_{0}>0$.

Un muy interesante hecho es que, además de la existencia de un enlace por debajo del acoplamiento del Higgs como en el caso de arriba, un análisis directo de la solución cerca del borde $u=0$ produce también un enlace por arriba. Nosotros también encontramos la existencia de un valor crítico $\lambda_{0}^{c}$ definido por,

$$
\frac{\lambda_{0}^{c}}{\hat{m}_{W}^{2}}=2+\left(\kappa_{1}-1\right)\left(2 \kappa_{1}+3\right)
$$

tal que para $\lambda_{0}>\lambda_{0}^{c}$ la solución condensada deja de existir. En el ejemplo considerado arriba $\hat{m}_{W}{ }^{2}=1$, $\lambda_{0}^{c} \sim 5.854$. Esto es para ambos casos, isotrópico y anisotrópico. 

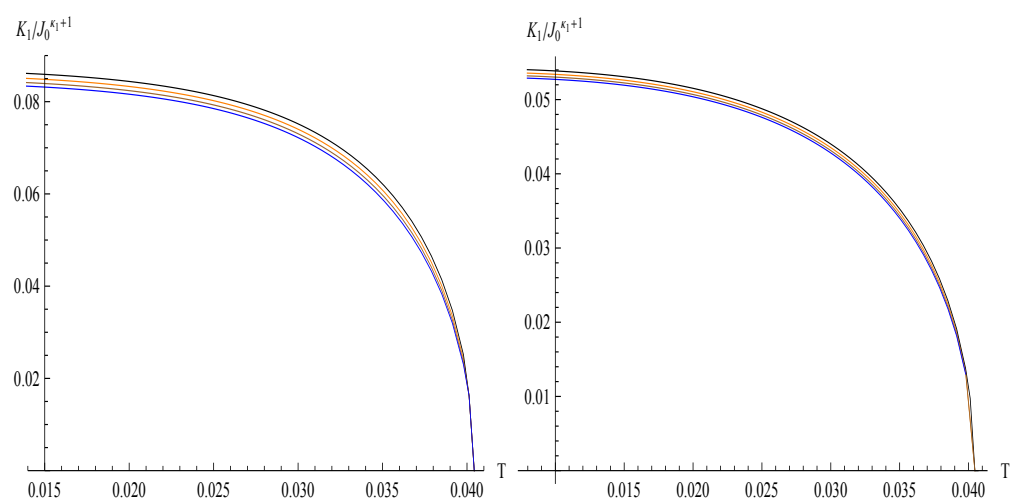

Figura 36: El condensado $K_{1}$ como función de la temperatura $T$, para $m_{W}^{2}=1$ y $\lambda_{0}=0$ (negra) 0.25 (naranja), 0.5 (marrón),0.75 (azul), en los casos anisotrópicos (izquierda) y isotrópicos (derecha).

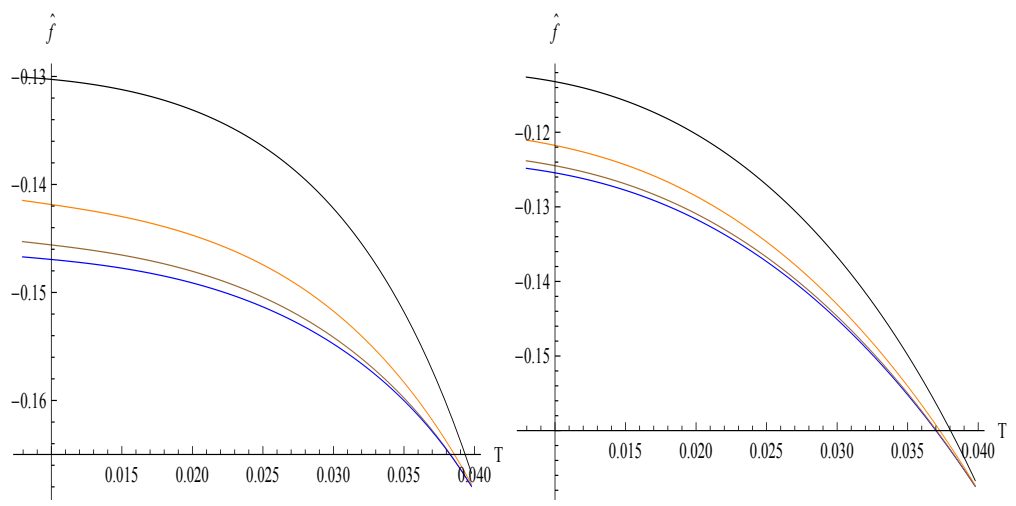

Figura 37: La energía libre $\hat{f}$ como función de la temperatura $T$ para $m_{W}{ }^{2}=1$ y $\lambda_{0}=0$ (negra), 0.25 (naranja), 0.5 (marrón), 0.75 (azul) en el caso anisotrópico (izquierda) y isotrópico (derecha).
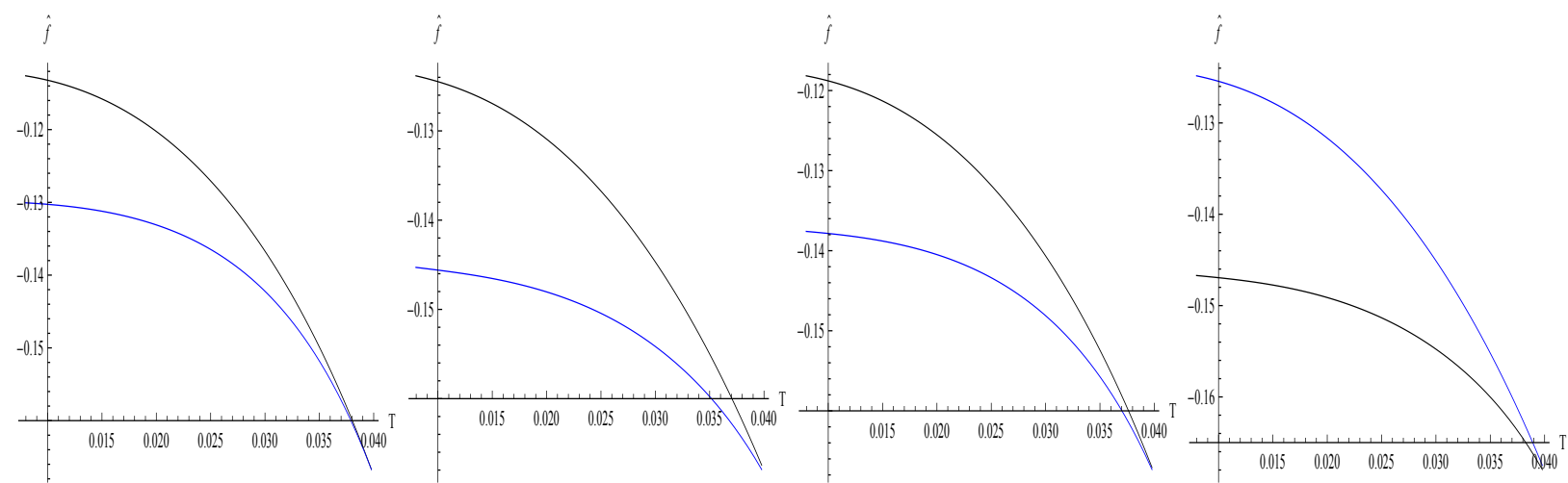

Figura 38: Comparación entre las energías libres en los casos anisotrópico (azul) y isotrópico (negra) para $m_{W}^{2}=1 \mathrm{y}$, de izquierda a derecha, $\lambda_{0}=0,0.25,0.5,0.75$. 


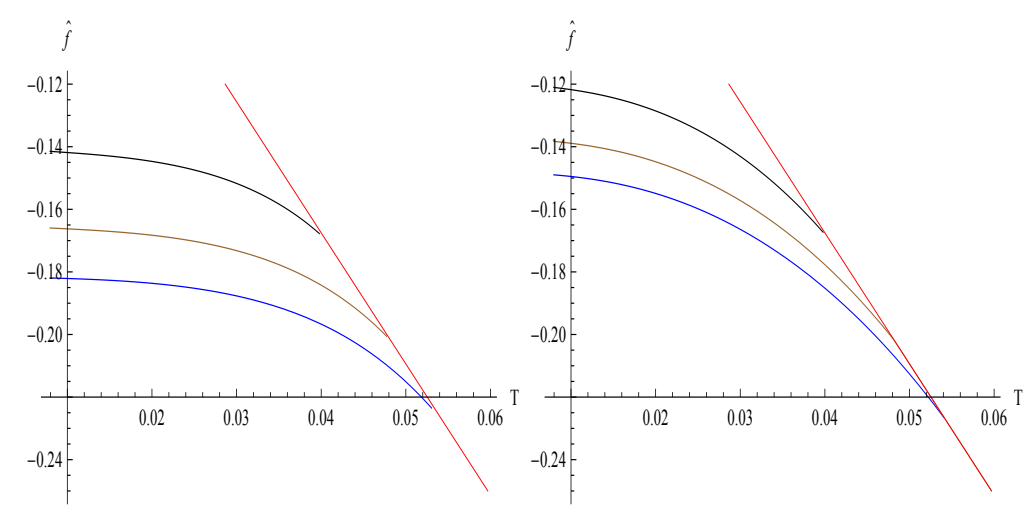

Figura 39: La energía libre como función de la temperatura en el caso anisotrópico (izquierda) y isotrópico (derecha) para un valor fijo de $\lambda_{0}=0.25$ y diferentes valores de $\hat{m}_{W}=0.5$ (azul), 0.7 (marrón) y 1.0 (negro). La curva roja representa la energía libre de la fase normal.

Las figuras 36 y 37 muestran el condensado y la energía libre respectivamente como función de la temperatura, para un valor fijo de $\hat{m}_{W}$ y diferentes valores del acoplamientos del Higgs ${ }^{17}$.

Uno pude apreciar que ambos, el parámetro de orden y la energía libre decrecen con el incremento de la fuerza del potencial $\lambda_{0}$; no obstante la temperatura crítica no cambia y las transiciones de fase permanecen de segundo orden.

En la figura 38 las energías libres para los casos isotrópicos y anisotrópicos son mostradas juntas como función de la temperatura para compararlas, para una escala de Higgs fija y diferentes acoplamientos del Higgs. La fase anisotrópica siempre permanece energéticamente favorecida sobre la fase isotrópica

Como se dijo antes, un valor crítico (280) arriba del cual la fase condensada no existe esta presente y fue verificado por nuestros cálculos numéricos; tales resultados ocurren en ambos casos isotrópicos y anisotrópicos

La figura 39 muestra las energías libres en función de la temperatura para un valor fijo de $\lambda_{0}$, para diferentes masas del bosón de calibre. Es observado que ellas se incrementan con el incremento de $\hat{m}_{W}$. Las curvas son similares a las curvas de la izquierda de las figuras 30 y 31 .

\subsection{Conclusiones y perspectivas}

En este trabajo hemos investigado soluciones en 4 dimensiones de agujeros negros con pelos no abelianos $S U(2)$ introducido por bosones de calibre de Yang-Mills y un campo de Higgs no trivial en la representación adjunta, cuyo v.e.v desencadena el rompimiento de la simetría de calibre a un subgrupo $U(1)$ bajo el cual el agujero negro esta cargado.

En el espíritu de la correspondencia AdS/CFT, la solución simétrica dada por el agujero negro AdS-RN cuando la temperatura es positiva y AdS cuando $T=0$, describe la fase no condensada de la QFT dual de tres dimensiones. Una solución con pelo no abeliano genéricamente rompe la simetría de calibre $U(1)$ junto con la simetría rotacional, y esto es interpretado como la fase condensada de la QFT. El parámetro de orden es el coeficiente del término de segundo orden (normalizable) de la componente magnética del campo de calibre, luego los sistemas son descriptos genéricamente como superconductores o superfluidos de onda p. Nosotros hemos considerado dos casos. El caso isotrópico que describe superconductores de onda $p+i p$ donde el subgrupo diagonal de $U(1)_{\text {calibre }} \times S O(2)_{\text {rot }}$ es preservado, y el caso anisotrópico donde ninguna simetría es preservada. En ambos casos obtenemos transiciones de fase a la temperatura crítica que decrecen cuando el acoplamiento gravitacional crece y en el caso de superconductores anisotrópicos las transiciones de fase se convierten de primer orden cuando el acoplamiento gravitacional es grande [34]. Estos resultados son resumidos en los diagramas de fase presentados en la figura 25.

Hemos también encontrado soluciones que describen estados bases de entropía nula del superconductor de onda $p$, mostrando la existencia de transiciones desde la fase normal (descripta por el espacio AdS) a la fase condensada, que esta presente debajo de cierto valor crítico del acoplamiento gravitacional $\tilde{\alpha}$. Estas transiciones de fase son de segundo orden, en acuerdo al comportamiento (276) del parámetro de orden cerca de la transición, obtenida de la figura 35. Tales estados son descriptos por geometrías de pared de dominio, que interpolan entre dos espacios AdS. La aparición del espacio AdS cerca del horizonte, con la misma escala

\footnotetext{
${ }^{17}$ Para valores más altos del acoplamientos del Higgs por encima del valor crítico, las curvas no experimentan cambios significativos.
} 
que el espacio AdS que aparece en el borde pero con diferentes velocidades de la luz, presumiblemente indica que hay un escala invariante emergente en el límite de $T=0$ [34] [35].

Finalmente estudiamos el efecto de considerar un potencial de Higgs no nulo. Fue encontrando que para acoplamientos constantes del Higgs mas grandes que el valor crítico $\lambda_{c}$, la solución colapsa a la fase normal. Este efecto depende del comportamiento ultravioleta del sistema; en particular es independiente de si el acoplamiento gravitacional es considerado o no. Para $\lambda$ debajo $\lambda_{c}$ el sistema y su variables termodinámicas se comportan cualitativamente como en el caso $\lambda=0$, pero con una energía libre mas baja.

Un muy importante hecho conjeturado en la literatura [24] [9] [40] para sistemas sin campos de Higgs que nosotros contestamos explícitamente en este artículo incluyendo un correspondiente potencial de Higgs, es que debajo de cierta temperatura crítica en todo el espacio de los parámetros encontramos que la densidad de energía libre de la solución anisotrópica es mas baja que la del caso isotrópico, indicando que el superconductor de onda $\mathrm{p}$ es mas estable que el superconductor de onda $p+i p$. Esto es ilustrado en las figuras 32,33 y 38 .

Creemos que es necesario hacer las siguientes aclaraciones. Resulta directo ver que si apagamos el campo de Higgs las e.o.m del superconductor de onda p son recuperadas. No obstante si apagamos la parate magnética del campo de calibre, $K_{1}=K_{2}=0$, se ve que no recuperamos las e.o.m de un superconductor de onda $s$. Esto es debido al hecho de que encendimos el campo de Higgs en la dirección $X_{0}$, y no en la dirección $X_{1}$. Esto nos lleva a concluir que en nuestro modelo el campo de Higgs no condensa espontánemante ya que la componente temporal del campo de calibre (cual juega un rol fundamental en la condensación) es nulo. Por lo tanto en nuestro esquema nunca tendremos una competición entre las fases de onda s y p como en los casos analizados en las referencias [26]-[29] donde el ansatz para el campo de materia es ligeramente diferente y el valor del vev para el campo de Higgs es puesto a cero. Por lo tanto no es difícil ver de las e.o.m que una configuración donde un vev (257) implica necesariamente un campo de Higgs no trivial; no obstante el vev de su campo escalar dual $\mathcal{O}(x)$,

$$
\langle\mathcal{O}(x)\rangle \sim H_{1}
$$

no indica ningún rompimiento espontáneo de simetría en vista de la presencia de la fuente $H_{0}$.

Debemos destacar que, aunque ellos comparten algunas características similares, la presencia del campo de Higgs con una no trivial b.c $|\vec{H}(\infty)|=H_{0}>0$, introduce una escala que hace a nuestro sistema diferente de aquellos considerados [9] [48], en los cuales los escalares no están presentes. Por otro lado del hecho obvio que el sistema es mas grande y mas complexo; en particular nosotros tenemos tres parámetros libres $\left(\alpha, \hat{m}_{W}, J_{0}\right)$ y otras cosas más, como por ejemplo la dimensión (259) del parámetro de orden permanece arbitrario. En efecto, en los sistemas del tipo Einstein-Yang-Mills donde el campo de Higgs no esta presente, la temperatura es solo una función del parámetro $\alpha \equiv \frac{\kappa}{e L}$ [34]. Por otro lado y mas importante, desde el punto de vista de la QFT para la cual los sistemas que hemos considerados tiene un dual holográfico. Este hecho puede ser elucidado estudiando las propiedades de transporte del sistema i.e las conductividades. Incluso ignorando los efectos del acoplamiento gravitacional, terminamos con un sistema de quince ecuaciónes de segundo orden acopladas que resultan mucho mas pesado para desacoplarlas que en el caso de no tener campo de Higgs. Esperamos reportar resultados en esta dirección en el futuro cercano [54].

\subsection{Apéndice A1: expansiones en el borde}

A lo largo de este artículo usamos métodos de disparo para obtener soluciones a las e.o.m. Presentamos aquí el (siguiente) orden de los campos cerca del borde, que es necesario para llevar a cabo los cálculos numéricos.

Para grandes $y \rightarrow \infty$ los campos admiten la expasión,

$$
\begin{aligned}
A(y) & =1+\frac{A_{1}}{y^{a_{1}}} \tilde{A}_{1}(y)=1+\frac{A_{1}}{y^{a_{1}}}\left(1+\frac{A_{2}}{y^{a_{2}}} \tilde{A}_{2}(y)\right) \\
f(y) & =y^{2}+\frac{F_{1}}{y} \tilde{F}_{1}(y)=1+\frac{F_{1}}{y}\left(1+\frac{\alpha^{2} J_{1}{ }^{2}}{2 \hat{m}_{W^{2}} F_{1} y} \tilde{F}_{2}(y)\right) \\
c(y) & =1+\frac{C_{1}}{y^{3}} \tilde{C}_{1}(y)=1+\frac{C_{1}}{y^{3}}\left(1+\frac{C_{2}}{y^{c_{2}}} \tilde{C}_{2}(y)\right) \\
K(y) & =\frac{K_{1}}{y^{\kappa_{1}}} \tilde{K}_{1}(y)=\frac{K_{1}}{y^{\kappa_{1}}}\left(1-\frac{J_{0}{ }^{2}}{2\left(1+2 \kappa_{1}\right) y^{2}} \tilde{K}_{2}(y)\right) \quad ; \quad \kappa_{1} \equiv \frac{1}{2}+\sqrt{\frac{1}{4}+\hat{m}_{W}} \\
J(y) & =J_{0}+\frac{J_{1}}{y} \tilde{J}_{1}(y)=J_{0}+\frac{J_{1}}{y}\left(1+\frac{J_{2}}{y^{j_{2}}} \tilde{J}_{2}(y)\right) \\
H(y) & =1+\frac{H_{1}}{y^{3}} \tilde{H}_{1}(y)=1+\frac{H_{1}}{y^{3}}\left(1+\frac{H_{2}}{y^{h_{2}}} \tilde{H}_{2}(y)\right)
\end{aligned}
$$


donde $\tilde{A}_{i}(0)=1, a_{i}>0$, etc., para $i=1,2, \ldots$ Las constantes $\left(F_{1}, C_{1}, J_{0}, J_{1}, K_{1}, H_{1}\right)$ son libres, el resto son determinadas por las e.o.m ${ }^{18}$ En el caso isotrópico, $K(y) \equiv K_{1}(y)=K_{2}(y), C(y)=1\left(C_{1}=0\right)$ son dadas por,

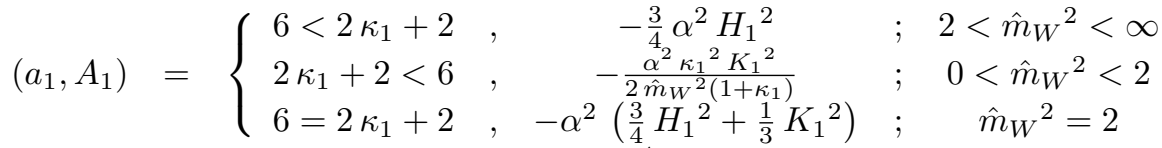

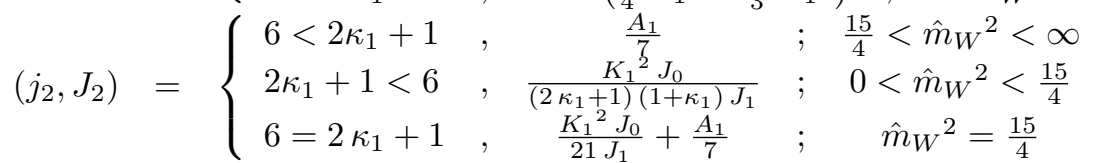

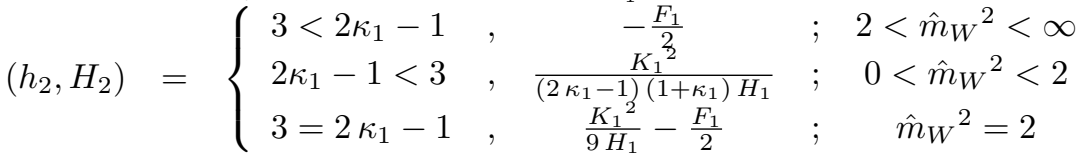

mientras que en el caso anisotrópico, $K(y) \equiv K_{1}(y), K_{2}(y)=0$, resultan,

$$
\begin{aligned}
& \left(a_{1}, A_{1}\right)=\left(3,-C_{1}\right)
\end{aligned}
$$

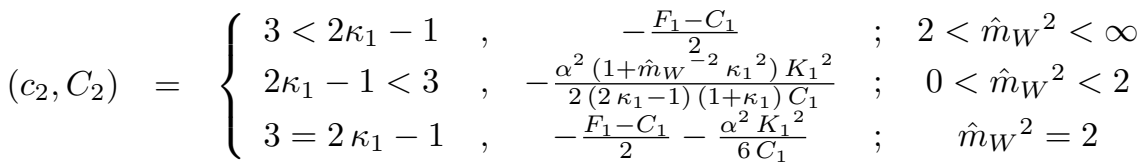

$$
\begin{aligned}
& \left(j_{2}, J_{2}\right)=\left(3,-\frac{C_{1}}{2}\right)
\end{aligned}
$$

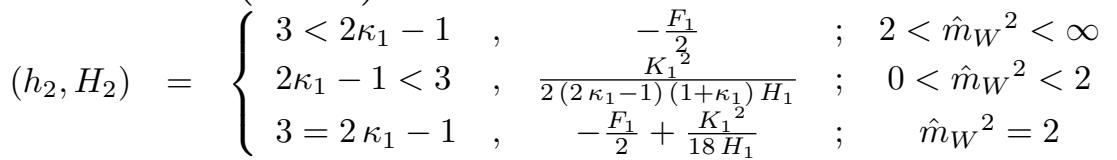

\subsection{Apéndice A2: convenciones}

En este apéndice escribimos las e.o.m en las convenciones de las referencias [34], [55]. Además de estar mas a menudo en la literatura, ellas suelen ser convenientes en algunos cálculos numéricos.

$\mathrm{El}$ ansatz es escrito como,

$$
\begin{aligned}
g & =\frac{L^{2}}{u^{2}}\left(-\tilde{f}(u) s(u)^{2} d t^{2}+\frac{d x^{2}}{g(u)^{2}}+g(u)^{2} d \tilde{y}^{2}+\frac{d u^{2}}{\tilde{f}(u)}\right) \\
A & =d t \tilde{J}(u) X_{0}+d x \tilde{K}_{1}(u) X_{1}+d \tilde{y} \tilde{K}_{2}(u) X_{2} \\
H & =H_{0} \tilde{H}(u) X_{0}
\end{aligned}
$$

La relación con las convenciones usadas en la métrica de este trabajo son,

$$
\begin{aligned}
& x^{0}=L t \quad, \quad x^{1}=L x \quad, \quad x^{2}=L \tilde{y} \quad, \quad y=\frac{g(u)}{u} \\
& c(y)=\frac{1}{g(u)^{2}} \quad, \quad f(y)=\frac{\tilde{f}(u)}{u^{2}}\left(g(u)-u g^{\prime}(u)\right)^{2} \quad, \quad A(y)=\frac{s(u)}{g(u)-u g^{\prime}(u)} \\
& J(y)=\tilde{J}(u) \quad, \quad K_{1}(y)=K_{1}(u) \quad, \quad K_{2}(y)=\tilde{K}_{2}(u) \quad, \quad H(y)=\tilde{H}(u)
\end{aligned}
$$

Las e.o.m de la gravedad resultan (sin los tildes),

$$
\begin{aligned}
f^{\prime}(u) & =\frac{3}{u}(f(u)-1)+u f(u) \frac{g^{\prime}(u)^{2}}{g(u)^{2}}+\frac{u}{g(u)^{2}}(1) \\
\frac{s^{\prime}(u)}{s(u)} & =-u \frac{g^{\prime}(u)^{2}}{g(u)^{2}}-\frac{u^{3}}{g(u)^{2}} y^{\prime}(u)^{2}(2) \\
2 \frac{u^{2} g(u)}{f(u) s(u)}\left(\frac{s(u) f(u)}{u^{2} g(u)} g^{\prime}(u)\right)^{\prime} & =-\frac{1}{f(u) g(u)}(3)
\end{aligned}
$$

donde (1), (2), (3) son las r.h.s.'s de (241) escritas en las variables (287), mientras que las e.o.m de la materia son,

$$
\frac{1}{g(u)^{2}}\left(f(u) s(u) g(u)^{2} K_{1}^{\prime}(u)\right)^{\prime}=\left(\frac{s(u)}{g(u)^{2}} K_{2}(u)^{2}+\hat{m}_{W}{ }^{2} \frac{s(u) H(u)^{2}}{u^{2}}-\frac{J(u)^{2}}{f(u) s(u)}\right) K_{1}(u)
$$

\footnotetext{
${ }^{18}$ Las condiciones de borde en el horizonte deja solo un parámetro libre (que lo tomaremos como $J_{0}$ ) que determina completamente la solución, ver sección 5.4.
} 


$$
\begin{aligned}
\frac{1}{g(u)^{2}}\left(f(u) s(u) g(u)^{2} K_{2}^{\prime}(u)\right)^{\prime} & =\left(s(u) g(u)^{2} K_{1}(u)^{2}+\hat{m}_{W}{ }^{2} \frac{s(u) H(u)^{2}}{u^{2}}-\frac{J(u)^{2}}{f(u) s(u)}\right) K_{2}(u) \\
f(u) s(u)\left(\frac{J^{\prime}(u)}{s(u)}\right)^{\prime} & =\left(g(u)^{2} K_{1}(u)^{2}+\frac{K_{2}(y)^{2}}{g(u)^{2}}\right) J(u) \\
\frac{u^{2}}{s(u)}\left(\frac{f(u) s(u)}{u^{2}} H^{\prime}(u)\right)^{\prime} & =\left(g(u)^{2} K_{1}(u)^{2}+\frac{K_{2}(y)^{2}}{g(u)^{2}}+\frac{\lambda_{0}}{\hat{m}_{W^{2}}} \frac{H(u)^{2}-1}{u^{2}}\right) H(u)
\end{aligned}
$$

Finalmente, las contribuciones a la densidad de energía libre son,

$$
\begin{aligned}
\hat{f}^{(b u l k)} & =\frac{\alpha^{2}}{2 J_{0}^{3}} \int_{u_{0}}^{u_{1}} d u s(u)\left(\frac{6}{\alpha^{2} u^{4}}-\frac{\lambda_{0}}{2 u^{4}}\left(H(u)^{2}-1\right)^{2}\right. \\
& +\frac{f(u)}{g(u)^{2}}\left(g(u)^{4} K_{1}^{\prime}(u)^{2}+K_{2}^{\prime}(u)^{2}\right)+K_{1}(u)^{2} K_{2}(u)^{2} \\
& \left.-\frac{J^{\prime}(u)^{2}}{s(u)^{2}}-\frac{J(u)^{2}}{f(u) s(u)^{2} g(u)^{2}}\left(g(u)^{4} K_{1}(y)^{2}+K_{2}(y)^{2}\right)\right) \\
\hat{f}^{(G H)} & =\left.\frac{\alpha^{2}}{2 J_{0}^{3}} \frac{2}{\alpha^{2}} u f(u)^{\frac{1}{2}}\left(\frac{f(u)^{\frac{1}{2}} s(u)}{u^{3}}\right)^{\prime}\right|_{u_{0}} \\
\hat{f}^{(c t)} & =\left.\frac{\alpha^{2}}{2 J_{0}{ }^{3}} \frac{4}{\alpha^{2}}\left(\frac{f(u)^{\frac{1}{2}} s(u)}{u^{3}}\right)\right|_{u_{0}}
\end{aligned}
$$




\title{
6 Funciones espectrales de superconductores holográficos de onda- $p$
}

\begin{abstract}
Resumen
Investigamos la función espectral de fermiones en un superconductor onda-p, tanto a temperatura finita como a acoplamiento gravitacional finito, usando la correspondencia $A d S / C F T$ y extendiendo investigación previa. Hemos encontrado que, para cualquier acoplamiento debajo de un cierto valor crítico, el sistema se comporta como su límite de temperatura cero. Incrementando el acoplamiento, la estructura del tipo "pico-depresión-joroba" que caracteriza la función espectral, a momento finito desaparece. En la región donde la transición de fase del estado normal al superconductor es de primer orden, la presencia de un parámetro de orden no nulo es reflejado en la ausencia de simetría rotacional en la función espectral a la temperatura crítica.
\end{abstract}

\subsection{Introducción}

Los superconductores son clasificados de acuerdo a la simetría de su función salto de energía $\Delta$ [57]. Desde este punto de vista, los superconductores convencionales BCS corresponden a simetrías del tipo $s$. Con respecto a los HTSC, los cupratos se cree que tienen simetría de onda $d$, mientras que algunos rutenatos como $\mathrm{Sr}_{2} \mathrm{RuO}_{4}$ se suponen que tienen simetría de onda $p$.

En este contexto, un modelo de superconductor a temperatura y potencial químico finito con un campo dinámico cargado, es necesario para describir el condensado . Con esto, se espera que para altas temperaturas la geometría del espacio-tiempo sea un agujero negro cargado asintóticamente AdS, este será dual a la fase normal de superconductor, mientras que a bajas temperaturas el agujero negro podría adquirir pelo del campo que modela al condensado. Esto es, en efecto, lo que sucede en los conocidos modelos de los superconductores holográficos.

Por ejemplo, los superconductores holográficos de onda $s$ son modelados añadiendo un campo escalar cargado en el espacio curvo, este es dual al condensado escalar que vive en la teoría de campos. Debajo de cierta temperatura crítica una solución con pelo escalar no trivial aparece, la cual tiene menos energía libre que la solución AdS Reissner-Nördstrom que describe el estado o fase normal del superconductor. Este pelo del agujero negro rompe la simetría de calibre $U(1)$ y , de acuerdo a la correspondencia $A d S / C F T$, esto es interpretado en la teoría de campo como un rompimiento espontáneo de la simetría global $U(1)$ debido al condensado escalar [7][8][60][61][62].

En este artículo estamos interesados en los superconductores holográficos de onda $p$, donde el condensado es alguna componente de un campo vectorial. En orden, para que este se encuentre cargado, un campo de calibre no abeliano es requerido. En este, sistema la fase normal es una solución con una componente no trivial del campo eléctrico el alguna dirección del álgebra de calibre, representando la carga del BH AdS ReissnerNördstrom. Tal elección rompe el grupo de calibre a una simetría de calibre residual $U(1)$. Similarmente como en el caso del superconductor de onda $s$, la solución normal es energéticamente favorecida hasta que la temperatura crítica es alcanzada; debajo de la cual una solución con una componente magnética en otra dirección de calibre se convierte en no trivial, rompiendo espontánemanete la simetría de calibre residual $U(1)$ y la simetría espacial rotacional ${ }^{19}$. Esto es interpretado de acuerdo a la correspondencia AdS/CFT como la aparición de una nueva fase en la teoría del borde, con un condensado vectorial no trivial que espontáneamente rompe la simetría global $U(1)$ [9]. La inclusión del acoplamiento gravitacional de los campos de calibre produce un cambio en el orden de la transición de fase para un acoplamiento gravitacional crítico, pasando de una transición de segundo orden a una de primer orden [63][14][64][65].

Los fermiones holográficos en la fase normal fueron estudiados en las referencias [66][67][68][69][70] entre otras, las conductividades para estos sistemas fueron computadas en [71][72]. El esquema que emerge es la de un líquido de Fermi con una superficie de Fermi bien definida, con o sin cuasi-partícualas con gran vida media dependiendo de los parámetros de la teoría. Útiles referencias acerca de estos temas son [73][74]. Con respecto a la fase superconductora, los grados de libertad fermiónicos en el superconductor de onda- $s$ fueron estudiados en [13][75][76][77]. Por el lado del superconductor de onda- $p$, fueron primeros analizado en [14], para el caso de temperatura nula a un acoplamiento gravitacional finito. El caso de temperatura finita fue estudiado en el probe límite en [78]. El objetivo de este trabajo es extender el computo para incluir ambos efectos, el de un acoplamiento gravitacional finito y considerar temperatura finita [10].

El artículo es divido como sigue. En la sección 6.2 revisamos las soluciones que describen a un superconductor de onda- $p$, para temperatura nula y temperatura finita. La sección 6.5 es devota a el estudio

\footnotetext{
${ }^{19}$ Campos de Higgs pueden ser añadidos pero no lo haremos aquí. Tal solución isotrópica con $A_{x^{1}}^{1}=A_{x^{2}}^{2} \neq 0$ que preserva el subgrupo diagonal de $U(1)_{\text {calibre }} \times S O(2)_{\text {rotation }}$ puede ser obtenido. No obstante siempre tiene una energía libre mas alta que la solución anisotrópica como es mostrado explícitamente en la referencia [81].
} 
de las ecuaciónes de movimiento de los fermiones en la representación fundamental de $S U(2)$, el análisis de las condiciones de borde y el computo de las funciónes de correlación siguiendo los pasos de [14][80]. En la sección 6.8 presentamos los resultados numéricos, mientras que en la sección 6.9 es dejada a las discusiones y conclusiones. Dos apéndices con algunos resultados relevantes con respecto a la función espectral son añadidos en el final.

\subsection{Sector bosónico: el superconductor holográfico de onda- $p$}

En esta sección estableceremos nuestras convenciones y revisaremos la contrucción de soluciones duales al superconductor de onda - $p$ holográfico [9], que serán usadas en el resto del artículo. Primero nos concentraremos en encontrar numéricamente la solución geométrica del espacio-tiempo, y entonces daremos la interpretación en el borde.

\subsection{Solución gravitacional del espacio-tiempo}

Nuestro punto de partida es una teoría de la gravitación cuyo sector bosónico es una sistema que contiene gravedad mas una teoría de Yang-Mills en un espacio-tiempo de $3+1$ dimensiones, con una métrica $G_{M N}$ y $M, N=0,1,2,3$ con signatura $(-+++)$, y un grupo de calibre $S U(2)$ con generadores hermíticos $\tau^{a} \operatorname{con}$ $a=0,1,2$. La acción se escribe como

$$
S^{(b o s)}=\int d^{4} x \sqrt{|G|}\left(\frac{1}{2 \kappa^{2}}\left(R+\frac{6}{L^{2}}\right)-\frac{1}{4 e^{2}} F_{M N}^{a} F^{a M N}\right) .
$$

Aquí $\kappa^{2} \equiv 8 \pi G_{N}$ con $G_{N}$ la constantes de Newton, $L$ es la escala AdS relacionada a la constante cosmológica a través $\Lambda \equiv-3 / L^{2}$, e es el acoplamiento de calibre y la fuerza del campo de calibre es definido como $F_{M N}^{a} \equiv \partial_{M} A_{N}^{a}-\partial_{N} A_{M}^{a}+\epsilon_{a b c} A_{M}^{b} A_{N}^{c}$. Las ecuaciónes de movimiento derivadas de esta acción se escriben,

$$
\begin{aligned}
R_{M N}-\frac{R}{2} G_{M N}+\Lambda G_{M N} & =\frac{\kappa^{2}}{e^{2}}\left(F_{S M}^{a} F_{N}^{a S}-\frac{G_{M N}}{4} F_{P Q}^{a} F^{a P Q}\right), \\
\nabla_{S} F_{M N}^{a}+\epsilon_{a b c} A_{S}^{b} F_{M N}^{c} & =0,
\end{aligned}
$$

donde " $\nabla$ " es la derivada covariante geométrica usual. Nosotros estamos interesados en soluciones de la forma $[9][63][65][81]$

$$
\begin{aligned}
G & =\frac{L^{2}}{z^{2}}\left(-f(z) s(z)^{2} d t^{2}+\frac{d z^{2}}{f(z)}+\frac{d x^{2}}{g(z)^{2}}+g(z)^{2} d y^{2}\right), \\
\mathbf{A} & =\phi(z) d t \tau_{0}+W(z) d x \tau_{1} .
\end{aligned}
$$

Para entender este ansatz, primero observamos que una $\phi(z)$ no nula rompe el grupo de calibre $S U(2)$ hacia $U(1)_{\tau_{0}}$, y seleccionamos una dirección preferida temporal $t$. Entonces, una componente "magnética" no nula $W(z)$ rompe la simetría residual $U(1)_{\tau_{0}}$ completamente, y establece una anisotropía espacial entre $x$ y $y$. Si tenemos en cuenta los efectos del acoplamiento gravitacional el sistema no puede soportar una métrica rotacionalmente invariante, y esto es porque necesitamos incluir no solo $f(z)$ y $s(z)$ sino que también una eventual componente no trivial $g(z)$. Reemplacemos el ansatz en las ecuaciónes (292) y obtendremos lo siguiente

$$
\begin{aligned}
s\left(\frac{\phi^{\prime}}{s}\right)^{\prime} & =\frac{g^{2} W^{2}}{f} \phi \\
\left(s f g^{2} W^{\prime}\right)^{\prime} & =-\frac{g^{2} \phi^{2}}{f s} W \\
\frac{z^{3}}{s}\left(\frac{s f}{z^{3}}\right)^{\prime} & =-\frac{3}{z}+\frac{\lambda^{2}}{2} \frac{z^{3} \phi^{\prime 2}}{s^{2}} \\
s^{\prime} & =-z s \frac{g^{\prime 2}}{g^{2}}-\frac{\lambda^{2}}{2} z^{3} g^{2}\left(s W^{\prime 2}+\frac{\phi^{2}}{s f^{2}} W^{2}\right), \\
g^{\prime \prime}-\frac{g^{\prime 2}}{g} & =-\frac{g^{\prime}}{z}\left(1-\frac{3}{f}+\frac{\lambda^{2}}{2} \frac{z^{4} \phi^{\prime 2}}{f s^{2}}\right)+\frac{\lambda^{2}}{2} z^{2} g^{3}\left(W^{\prime 2}-\frac{\phi^{2} W^{2}}{s^{2} f^{2}}\right)
\end{aligned}
$$

donde hemos definido el acoplamiento adimensional ${ }^{20} \lambda \equiv \kappa /(L e)$.

${ }^{20}$ La constante de acoplamiento $g_{Y M}$ en [14] y la nuestra, están relacionadas por, $\lambda=1 /\left(\sqrt{2} g_{Y M}\right)$. 
Notemos que el ansatz (293) y las ecuaciónes de movimiento (294) son invariantes bajo las simetrías de escala

$$
\begin{aligned}
(t ; s, \phi) & \longrightarrow\left(\frac{t}{\alpha} ; \alpha s, \alpha \phi\right), \\
(x, y ; g, W) & \longrightarrow\left(\frac{x}{\beta}, \beta y ; \frac{g}{\beta}, \beta W\right), \\
\left(x^{\mu}, z ; \phi, W\right) & \longrightarrow\left(\frac{1}{\gamma} x^{\mu}, \frac{1}{\gamma} z ; \gamma \phi, \gamma W\right) .
\end{aligned}
$$

Estas simetrías resultaran útiles para fijar valores asintóticos de algunos de los campos.

Las expresiones representan un sistema de ecuaciónes diferenciales de segundo orden en la variable independiente $z$, la cual se mueve en un cierto intervalo $z \in\left[z_{U V}, z_{I R}\right]$. Esta debe ser resuelta con condiciones de borde en las fronteras $z_{U V}$ y $z_{I R}$ reflejan la situación física deseada. En lo que sigue, definiremos $z_{U V}=0$ como el borde AdS. Cerca de $z_{U V}$, las ecuaciónes de arriba puede ser expandidas en potencias de $z$ y resueltas, para obtener los comportamiento asintóticos

$$
\begin{aligned}
\phi(z) & =\mu+\rho z+\ldots, \\
W(z) & =W_{U V}+W_{U V}^{\prime} z+\ldots, \\
f(z) & =1+f_{3} z^{3}+\ldots, \\
s(z) & =1-\frac{\lambda^{2} W_{U V}^{\prime 2}}{8} z^{4}+\ldots, \\
g(z) & =1+g_{3} z^{3} \ldots,
\end{aligned}
$$

donde hemos usando las dos primeras simetrías en (295) para fijar las condiciones de normalización $s(0)=$ $g(0)=1$, en un camino para identificar las $x^{\mu}$ 's coordenadas de Minskowski en el infinito. La tercer simetría será usada de acuerdo a si estudiamos los casos de entropía nula o finita en las secciones que vendrán.

Ahora tomando $\mu \neq 0$ la condición de borde rompe explícitamente la simetría de calibre $S U(2)$ a un subgrupo $U(1)_{\tau_{0}}$. Luego poner $W_{U V}=0$, implica que no hay un rompimiento explícito de la simetría de calibre residual $U(1)_{\tau_{0}}$, entonces un valor no nulo de la cantidad $W_{U V}^{\prime}$ señala un rompimiento espontáneo del calibre restante $U(1)_{\tau_{0}}$ y la simetría rotacional

En el extremo opuesto de la geometría, el valor de $z_{I R}$ y los detalles de las expansiones asintóticas depende de si queremos estudiar una solución con una entropía nula o no nula. En cualquier caso la temperatura de Hawking de la solución es determinada por

$$
T=\frac{s\left(z_{I R}\right)\left|f^{\prime}\left(z_{I R}\right)\right|}{4 \pi} \equiv \frac{s_{I R}\left|f_{I R}^{\prime}\right|}{4 \pi}
$$

\subsubsection{Entropía de Beckenstein-Hawking nula}

En este caso, extendemos la geometría de la región $z_{I R} \rightarrow \infty$ para fijar nuestras condiciones de borde allí. En tal región, las ecuaciónes de movimiento dictan los siguientes comportamientos

$$
\begin{aligned}
\phi(z) & =\phi_{I R} e^{-g_{I R} w_{I R} z}+\ldots, \\
W(z) & =w_{I R}-\frac{\phi_{I R}^{2}}{4 s_{0}^{2} g_{I R}^{2} w_{I R}} e^{-2 g_{I R} w_{I R} z}+\ldots \\
f(z) & =1-\frac{\lambda^{2}}{2} \frac{\phi_{I R}^{2} g_{I R} w_{I R}}{s_{I R}^{2}} z^{3} e^{-2 g_{I R} w_{I R} z}+\ldots, \\
s(z) & =s_{I R}+\frac{\lambda^{2}}{4} \frac{\phi_{I R}^{2} g_{I R} w_{I R}}{s_{I R}} z^{3} e^{-2 g_{I R} w_{I R} z}+\ldots \\
g(z) & =g_{I R}-\frac{\lambda^{2}}{8} \frac{g_{I R} \phi_{I R}^{2}}{s_{I R}^{2}} z^{2} e^{-2 g_{I R} w_{I R} z}+\ldots
\end{aligned}
$$

En las expresiones de arriba imponemos suaves condiciones poniendo a cero los coeficientes de la parte divergente de las soluciones del sistema línealizado. Genéricamente, las soluciones con condiciones de borde (296) y (298) son paredes de dominio que interpolan entre dos espacio AdS con escalas iguales pero diferentes velocidades de la luz, y describen el verdadero vacío de la teoría. De acuerdo a (297), ellas tienen entropia cero a temperatura nula. Tales soluciones fueron primero estudiadas en [14][40] y revisadas mas recientemente en [81]. Nosotros fijaremos $\mu=1$ usando la ultima simetría de escala en (295). 
En la figura 40 las funciónes $f(z), g(z), W(z), \phi(z)$ y $s(z)$ son mostradas y se corresponden a diferentes valores del acoplamiento $\lambda$. Vemos que la simetría rotacional y de calibre están rotas por las funciónes $g(z)$ y $W(z)$. El comportamiento líneal de $W(z)$ en el borde señala el rompimiento de la simetría de calibre restante $U(1)_{\tau_{0}}$. Cuando $\lambda \rightarrow \lambda_{*} \approx 0.995$ (correspondiente a $g_{Y M *} \approx 0.71$ ) un horizonte a $z_{h} \approx 2.462$ se forma y la solución tiende a un BH extremo AdS-Reissner-Nördstrom ${ }^{21}$

$$
\begin{aligned}
W(z) & =0 \\
g(z) & =s(z)=1 \\
\phi(z) & =1-\frac{z}{z_{h}} \\
f(z) & =\left(1-\frac{z}{z_{h}}\right)^{2}\left(1+2 \frac{z}{z_{h}}+3\left(\frac{z}{z_{h}}\right)^{2}\right)
\end{aligned}
$$

Para los acoplamientos por encima del valor crítico cuántico $\lambda_{*}$ solo la solución AdS-Reissner-Nördstrom esta presente.
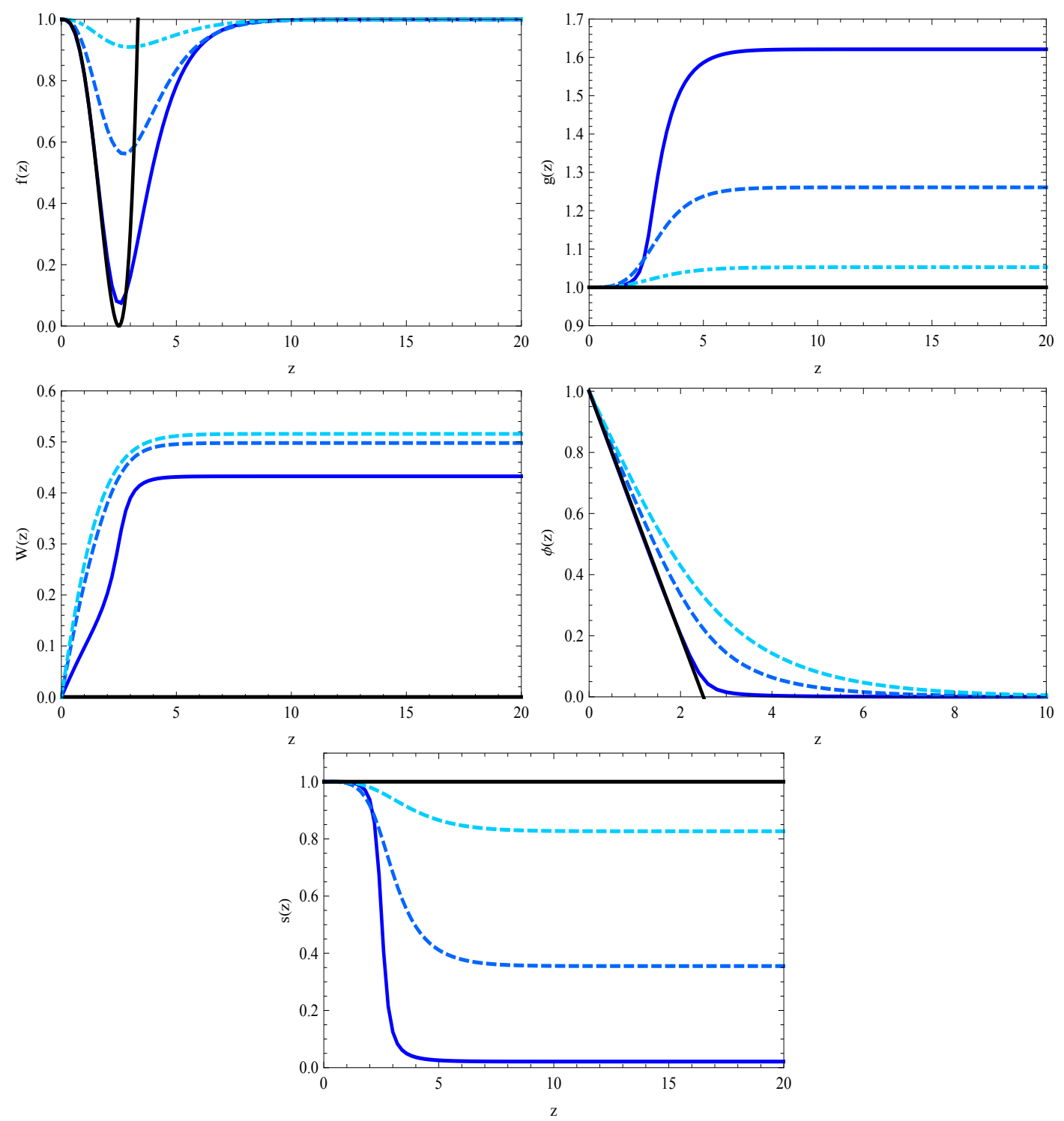

Figura 40: La métrica y el campo de calibre a entropía nula son mostradas, para diferentes valores de $\lambda$ : 0.40 (cyan), 0.8 (azul claro), 0.995 (azul). Las curvas negras corresponden a la solución del BH extremo cargado.

${ }^{21}$ Estrictamente hablando, el BH AdS cargado es definido para $0 \leq z \leq z_{h}$, ver la próxima sección 


\subsubsection{Entropía de Beckenstein-Hawking no nula}

Ahora consideramos soluciones con un horizonte $z_{I R}=z_{h}<\infty$ tal que $f\left(z_{h}\right) \equiv 0$. La expansión asintótica de la solución cerca del horizonte se comporta suavemente

$$
\begin{aligned}
\phi(z) & =\phi_{1}\left(z-z_{h}\right)+\ldots \\
W(z) & =W_{I R}+W_{I R}^{\prime}\left(z-z_{h}\right)+\ldots \\
f(z) & =f_{I R}^{\prime}\left(z-z_{h}\right)+\ldots \\
s(z) & =s_{I R}+s_{I R}^{\prime}\left(z-z_{h}\right)+\ldots \\
g(z) & =g_{I R}+g_{I R}^{\prime}\left(z-z_{h}\right)+\ldots
\end{aligned}
$$

Las condiciones de borde (296) y (300) son compatibles con la solución de un agujero negro AdS cargado

$$
\begin{aligned}
W(z) & =0 \\
g(z) & =s(z)=1 \\
\phi(z) & =\mu\left(1-\frac{z}{z_{h}}\right) \\
f(z) & =1-\left(1+Q^{2}\right)\left(\frac{z}{z_{h}}\right)^{3}+Q^{2}\left(\frac{z}{z_{h}}\right)^{4},
\end{aligned}
$$

donde $Q^{2} \equiv \lambda^{2} \mu^{2} z_{h}^{2} / 2$. La temperatura de Hawking de esta solución de agujero negro es dada por

$$
T=\frac{3-Q^{2}}{4 \pi z_{h}} .
$$

El límite de temperatura nula se corresponde al agujero negro AdS cargado extremo (entropía finita) con $Q^{2}=3$ considerado arriba en la ecuación (299). En efecto, la condición de extremo implica que la relación $\lambda_{*} z_{h}=\sqrt{6}$ se debe mantener, consistente con los valores numéricos encontrados, $\lambda_{*} \approx 0.995$ and $z_{h} \approx 2.462$.

Las condiciones (296) and (300) son también compatibles con una solución con pelo "magnético" $W(z) \neq$ 0 que rompe la simetría de calibre residual $U(1)_{\tau_{0}}$. Para obtener ésta, llevamos a cabo cálculos numéricos donde, usando la ultima simetría de escala en (295), fijamos la posición del horizonte al valor, $z_{h}=1$ [9][63].

En términos de la temperatura invariante de escala $T / \mu$, uno obtiene que la solución de agujero negro AdS cargado es energéticamente favorecida para temperaturas arriba de una temperatura crítica $T_{c}$. Para temperaturas mas bajas, una solución con pelo sin simetría es seleccionada. Como discutimos para diferentes modelos [61][82][83] uno puede interpretar este resultado declarando que un condensado es formado arriba del horizonte del agujero negro debido a un balance entre la fuerza gravitacional y electrostática.

\subsection{La interpretación del borde en términos de una QFT}

La invarianza de calibre $S U(2)$ de la acción (291) es realizada en el borde dual como una simetría interna global $S U(2)$. La presencia de un valor no nulo de $\phi(z)$ en el borde, es interpretado como un potencial químico finito $\mu$ para partículas cargadas bajo tal simetría, y además rompe la simetría global $S U(2)$ a la simetría residual global $U(1)_{\tau_{0}}$. La elección de la condición de borde $W_{U V}=0$ corresponde a apagar la fuente del operador dual $J_{x}^{1}$ en el lado de la QFT, cuyo valor de expectación es dado por $W_{U V}^{\prime}$. En consecuencia la cantidad $W_{U V}^{\prime}=\left\langle J_{x}^{1}\right\rangle$ puede ser considerada como el parámetro de orden de la QFT, un valor no nulo para el cual $W_{U V}^{\prime}=\left\langle J_{x}^{1}\right\rangle$ señala el rompimiento espontáneo de la simetría residual global $U(1)_{\tau_{0}}$ y la simetría rotacional. Una solución con un $W_{U V}^{\prime}$ no nulo es entonces interpretada como la fase dual de un superconductor de onda- $p$ [9]. Por otro lado, el estado normal del sistema es descripto por una solución de agujero negro AdS cargado (301) con $W_{U V}^{\prime}=0$.

La temperatura de Hawking de la solución de espacio curvo es interpretada como la temperatura del baño térmico en contacto con la teoría del borde. Ya que solución de un agujero negro cargado AdS es energéticamente favorecida para temperaturas por encima de $T_{c}$, podemos decir que la teoría dual esta en su fase normal para $T>T_{c}$, mientras que tiende a una fase anisotrópica superconductora para $T<T_{c}$. El valor de $T_{c}$ y el orden de la transición de fase depende del acoplamiento $\lambda$. En la figura 41(a) mostramos el diagrama de fases del superconductor de onda- $p$. Podemos distinguir tres regiónes del acoplamiento gravitacional, a saber

- Cuando $0 \leq \lambda \leq \lambda_{c} \approx 0.62$ el sistema tiene una transición de fase de segundo orden para algun valor de $T_{c}$ (línea critica azul), experimentando una solución con pelo para bajas temperaturas, mientras que para altas temperaturas se encuentra en la fase normal. 
- Cuando $\lambda_{c}<\lambda \leq \lambda_{*} \approx 0.995$ la transición de fase se convierte de primer orden (curva roja crítica). Notemos la presencia de dos curvas modales arriba y abajo de la linea crítica que señala la emergencia de la dos ramas de la solución con pelo $\left(T_{1}\right)$ y la desaparición de la rama inestable $\left(T_{2}\right)$ respectivamente.

- Para $\lambda>\lambda_{*}$ la fase de simetría rota deja de existir.

El parámetro de orden $W_{U V}^{\prime}$ y la energía libre son mostradas en las figuras 41(b) y 41(1)(2)(3) respectivamente, para tres diferentes acoplamiento típicos $\lambda=0.40<\lambda_{c}\left(\right.$ donde $\left.T_{c}=0.0467607 \mu\right), \lambda=0.62=\lambda_{c}$ (donde $\left.T_{c}=0.0252852 \mu\right)$ y $\lambda=0.80>\lambda_{c}\left(\right.$ donde $T_{c}=0.0103462 \mu$ ).

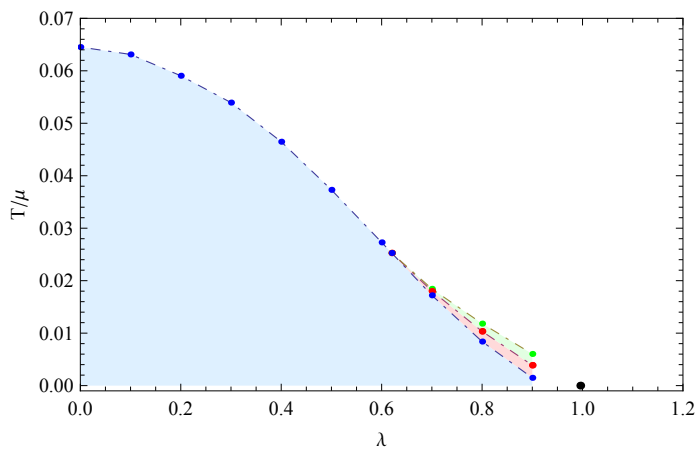

(a)

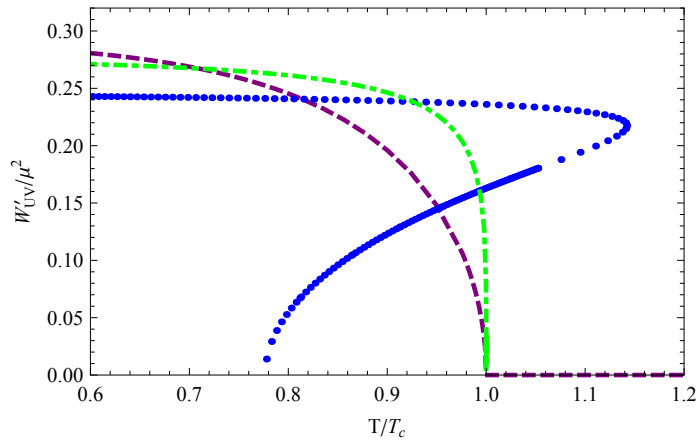

(b)

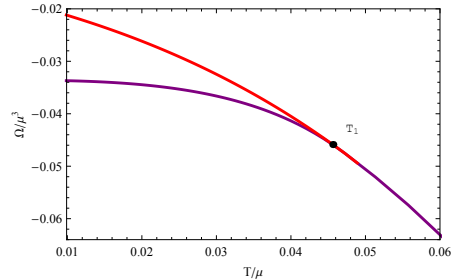

(1)

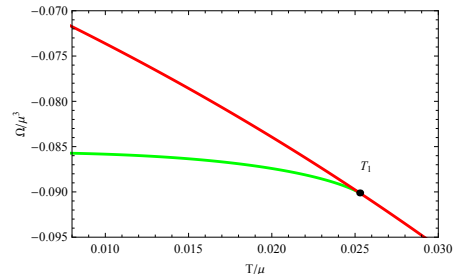

(2)

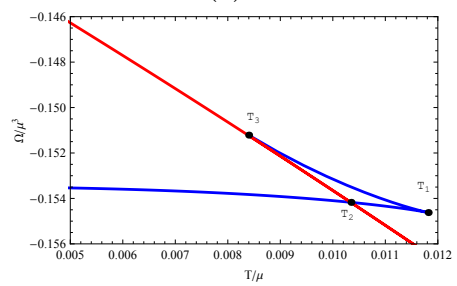

(3)

Figura 41: (a) Diagrama de fase de un superconductor de onda - $p$ en el plano $\lambda-T$. El punto negro indica el acoplamiento critico $\lambda_{*}$ a $T=0$ donde una transición de fase cuántica toma lugar. (b) El parámetro de orden como función de la temperatura para tres diferentes valores de $\lambda: \lambda=0.40<\lambda_{c}$ (violeta), $\lambda=0.62=\lambda_{c}$ (verde), $\lambda=0.80>\lambda_{c}$ (azul). (1) (2) (3) La energía libre para los valores de $\lambda$ como en (b); Las curvas rojas son las energías libres del sistema en la fase normal.

En lo que sigue, después de introducir los fermiones en esta geometría, analizaremos como su función espectral cambia en todo el diagrama de fases. 


\subsection{Sector fermiónico: añandiendo fermiones al superconductor holográfico de onda $p$}

Ahora vamos a movernos al sector fermiónico, introduciendo grados de libertad fermiónicos en la configuración definida arriba. Por lo pronto estaremos en el lado gravitacional y luego interpretaremos nuestros resultados numéricos desde el punto de vista de la QFT.

\subsection{Espinores de Dirac en un fondo gravitacional}

Consideraremos espinores de Dirac en la representación fundamental de $S U(2)$, definido por los generadores $\left\{\tau^{0}, \tau^{1}, \tau^{2}\right\}=\left\{\sigma^{3} / 2, \sigma^{1} / 2, \sigma^{2} / 2\right\}$ donde $\sigma_{1}, \sigma_{2}, \sigma_{3}$ son matrices de Pauli. Para acoplar estos espinores a la gravedad, primero debemos introducir un vielbein $\left\{\omega^{A}: G=\eta_{A B} \omega^{A} \omega^{B}\right\}$, junto con sus vectores bases duales $\left\{e_{A}: e_{A}\left(\omega^{B}\right)=\delta_{A}^{B}\right\}$, y una conexión de spin $\left\{\omega_{B}^{A}\right\}$, donde los índices locales corren de $A, B=0, \ldots, 3$. En la geometría (293) ellos se escriben

$$
\begin{aligned}
& \omega^{0} \equiv \frac{s \sqrt{f}}{z} d t, \quad e_{0} \equiv \frac{z}{s \sqrt{f}} \frac{\partial}{\partial t}, \\
& \omega^{1} \equiv \frac{1}{z g} d x, \quad e_{1} \equiv z g \frac{\partial}{\partial x}, \\
& \omega^{2} \equiv \frac{g}{z} d y, \quad e_{2} \equiv \frac{z}{g} \frac{\partial}{\partial y}, \\
& \omega^{3} \equiv \frac{1}{z \sqrt{f}} d z, \quad e_{3} \equiv z \sqrt{f} \frac{\partial}{\partial z} . \\
& \omega^{0}{ }_{3}=-\omega_{03}=+\omega_{30}=-\sqrt{f}\left(1-z \frac{s^{\prime}}{s}-\frac{z f^{\prime}}{2 f}\right) \omega^{0}, \\
& \omega^{1}{ }_{3}=+\omega_{13}=-\omega_{31}=-\sqrt{f}\left(1+z \frac{g^{\prime}}{g}\right) \omega^{1}, \\
& \omega^{2}{ }_{3}=+\omega_{23}=-\omega_{32}=-\sqrt{f}\left(1-z \frac{g^{\prime}}{g}\right) \omega^{2},
\end{aligned}
$$

las matrices gamma obedecen $\left\{\Gamma^{A}, \Gamma^{B}\right\}=2 \eta^{A B}$, y nos permiten a nosotros definir una derivada covariante como la usual

$$
\not D \Psi \equiv \Gamma^{A} D_{A} \Psi=\Gamma^{A}\left(e_{A}(\Psi)+\frac{1}{8} \omega_{A}^{B C}\left[\Gamma_{B}, \Gamma_{C}\right] \Psi-i A_{A}^{a} \tau_{a} \Psi\right),
$$

y introducir los proyectores sobre los autoestado de $\Gamma^{3}$ dados por $P_{ \pm} \equiv \frac{1}{2}\left(1 \mp \Gamma^{3}\right)$ con autovalores $\mp 1$. Es bien conocido que un termino de borde es necesario para tener un principio variacional bien definido [84][85]. Si fijamos los valores en el borde de los campos de Dirac para ser la parte izquierda (derecha) $\Psi_{+} \equiv P_{+} \Psi\left(\Psi_{-} \equiv P_{-} \Psi\right)$, la acción a ser considerada es

$$
S^{(f e r)}=-\int d^{4} x \sqrt{-G} \bar{\Psi}(\not D-m) \Psi \mp \int_{z \rightarrow 0} d^{3} x \sqrt{-h} \bar{\Psi}_{ \pm} \Psi_{\mp},
$$

donde $\bar{\Psi} \equiv \Psi^{\dagger} i \Gamma^{0} \quad$ y $h=h_{\mu \nu}(x) d x^{\mu} d x^{\nu} \quad$ es la métrica inducida sobre el borde. Las ecuaciónes de movimiento resultantes se escriben

$$
(\not D-m) \Psi=0
$$

para resolver estas ecuaciónes, encontramos conveniente trabajar en el espacio de momentos y re-escalear los espinores como $\Psi(x, z) \equiv\left(-\operatorname{det} G G^{z z}\right)^{-1 / 4} e^{i k_{\mu} x^{\mu}} \psi_{k}(z)=z^{3 / 2} f^{-\frac{1}{4}} s^{-\frac{1}{2}} e^{i k_{\mu} x^{\mu}} \psi_{k}(z)$ donde $\left(k_{\mu}\right)=$ $\left(-\omega, k_{x}, k_{y}\right)$. Usando la siguiente representación de las matrices $\Gamma$ de Dirac

$$
\Gamma^{\mu} \equiv\left(\begin{array}{cc}
0 & \gamma^{\mu} \\
\gamma^{\mu} & 0
\end{array}\right), \quad \Gamma^{3} \equiv\left(\begin{array}{cc}
-1 & 0 \\
0 & 1
\end{array}\right),
$$

donde las matrices $\gamma^{\mu}$ en un espacio $2+1$ son $\gamma^{0}=i \sigma_{2}, \gamma^{1}=\sigma_{1}, \gamma^{2}=\sigma_{3}$, podemos introducir dos espinores bidimensionales $\psi_{k}^{ \pm}(z)$ como $^{22}$

$$
\psi_{k}(z) \equiv\left(\begin{array}{c}
\psi_{k}^{+}(z) \\
\psi_{k}^{-}(z)
\end{array}\right)
$$

Las ecuaciones de movimiento (306) se escriben entonces como

\footnotetext{
${ }^{22}$ Los generadores del subgrupo de Lorentz en $2+1$ dimensiones son$$
\frac{i}{4}\left[\Gamma_{0}, \Gamma_{1}\right]=\frac{i}{2}\left(\begin{array}{cc}
\sigma_{3} & 0 \\
0 & \sigma_{3}
\end{array}\right), \quad \frac{i}{4}\left[\Gamma_{0}, \Gamma_{2}\right]=\frac{i}{2}\left(\begin{array}{cc}
-\sigma_{1} & 0 \\
0 & -\sigma_{1}
\end{array}\right), \quad \frac{i}{4}\left[\Gamma_{1}, \Gamma_{2}\right]=\frac{1}{2}\left(\begin{array}{cc}
\sigma_{2} & 0 \\
0 & \sigma_{2}
\end{array}\right),
$$

las cuales muestran la reductibilidad de la representación con respecto a $\operatorname{spin}(1,2)$, siendo $i \sigma_{3} / 2$ y $-i \sigma_{1} / 2$ los generadores de los boosts y $\sigma_{2} / 2$ el generador de las rotaciones en el plano $(x-y)$-. Los espinores de color izquierdos/derechos $\psi_{k}^{ \pm}(z)$ son entonces espinores de Dirac en la teoría del borde.
} 


$$
\begin{aligned}
& +\psi_{k}^{+\prime}+\frac{i}{\sqrt{f}}\left[\frac{1}{s \sqrt{f}}\left(\omega+\phi \tau_{0}\right) \gamma^{0}-g\left(k_{x}-W \tau_{1}\right) \gamma^{1}-\frac{k_{y}}{g} \gamma^{2}\right] \psi_{k}^{-}=-\frac{m}{z \sqrt{f}} \psi_{k}^{+}, \\
& -\psi_{k}^{-\prime}+\frac{i}{\sqrt{f}}\left[\frac{1}{s \sqrt{f}}\left(\omega+\phi \tau_{0}\right) \gamma^{0}-g\left(k_{x}-W \tau_{1}\right) \gamma^{1}-\frac{k_{y}}{g} \gamma^{2}\right] \psi_{k}^{+}=-\frac{m}{z \sqrt{f}} \psi_{k}^{-}
\end{aligned}
$$

Ahora podemos hacer explícita los índices de color escribiendo $\psi_{k}^{ \pm}=\left(\psi_{k}^{ \pm \alpha i}\right)$ donde $\alpha=1,2$ y $i=1,2$ son índices de espinores en el borde y índices de color respectivamente. Será conveniente en lo que sigue introducir las cuatro-uplas $\vec{u}_{k}(z)$ y $\vec{v}_{k}(z)$ como

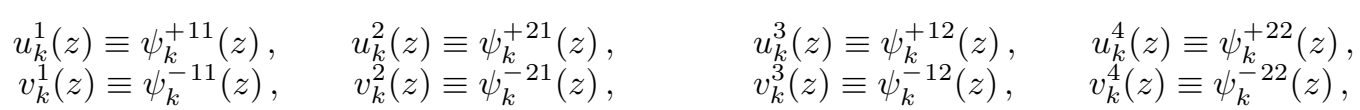

y la matriz real de $4 \times 4$

$$
\mathbf{U}(z) \equiv \frac{1}{\sqrt{f}}\left(\begin{array}{cccc}
-\frac{k_{y}}{g} & \frac{\left(\omega+\frac{\phi}{2}\right)}{s \sqrt{f}}-g k_{x} & 0 & g W / 2 \\
-\frac{\left(\omega+\frac{\phi}{2}\right)}{s \sqrt{f}}-g k_{x} & \frac{k_{y}}{g} & g W / 2 & 0 \\
0 & g W / 2 & -\frac{k_{y}}{g} & \frac{\left(\omega-\frac{\phi}{2}\right)}{s \sqrt{f}}-g k_{x} \\
g W / 2 & 0 & -\frac{\left(\omega-\frac{\phi}{2}\right)}{s \sqrt{f}}-g k_{x} & \frac{k_{y}}{g}
\end{array}\right) .
$$

En términos de esta notación, las ecuaciónes (309) son escritas en forma compacta como

$$
\begin{aligned}
& +\vec{u}_{k}^{\prime}(z)+i \mathbf{U}(z) \vec{v}_{k}(z)=0, \\
& -\vec{v}_{k}^{\prime}(z)+i \mathbf{U}(z) \vec{u}_{k}(z)=0,
\end{aligned}
$$

donde ponemos $m=0$ ya que nos concentraremos en este caso particular en el resto del artículo. Estas ecuaciónes necesitan ser resueltas para las ocho funciónes $\vec{u}_{k}(z), \vec{v}_{k}(z)$. Ya que ellas son ecuaciónes de primer orden, las soluciones son escritas en términos de ocho constantes no determinadas. Tales constantes son fijadas eligiendo condiciones de borde en la frontera $z_{U V}=0$ y $z_{I R}$ del dominio de la variable $z$. Nosotros hacemos la elección de fijar la parte izquierda $\Psi_{+}$en el borde, esto es $\vec{u}_{k}(z)$, siendo la acción como esta escrita en (305) con el signo de arriba. Entonces, cuando imponemos las condiciones de borde en $z_{I R}$, cuatro de estas constantes pasan a estar determinadas. .

Las ecuaciónes (312) pueden ser resueltas iterativamente en la vecindad de cualquier punto $z_{0}$ escribiendo

$$
\begin{aligned}
& \vec{u}_{k}(z)=\mathcal{C}\left(z ; z_{0}\right) \vec{u}_{k}^{(0)}-i \mathcal{S}\left(z ; z_{0}\right) \vec{v}_{k}^{(0)} \\
& \vec{v}_{k}(z)=i \mathcal{S}\left(z ; z_{0}\right) \vec{u}_{k}^{(0)}+\mathcal{C}\left(z ; z_{0}\right) \vec{v}_{k}^{(0)},
\end{aligned}
$$

donde

$$
\begin{aligned}
& \mathcal{C}\left(z ; z_{0}\right)=\mathrm{P} \cosh \left(\int_{z_{0}}^{z} d z^{\prime} \mathbf{U}\left(z^{\prime}\right)\right) \equiv \frac{1}{2}\left(\mathrm{P} e^{\int_{z_{0}}^{z} d z^{\prime} \mathbf{U}\left(z^{\prime}\right)}+\mathrm{P} e^{-\int_{z_{0}}^{z} d z^{\prime} \mathbf{U}\left(z^{\prime}\right)}\right) \\
& \mathcal{S}\left(z ; z_{0}\right)=\mathrm{P} \sinh \left(\int_{z_{0}}^{z} d z^{\prime} \mathbf{U}\left(z^{\prime}\right)\right) \equiv \frac{1}{2}\left(\mathrm{P} e^{\int_{z_{0}}^{z} d z^{\prime} \mathbf{U}\left(z^{\prime}\right)}-\mathrm{P} e^{-\int_{z_{0}}^{z} d z^{\prime} \mathbf{U}\left(z^{\prime}\right)}\right)
\end{aligned}
$$

Aquí P implica para cada orden. Los operadores de evolución $\mathcal{C}\left(z ; z_{0}\right)$ y $\mathcal{S}\left(z ; z_{0}\right)$ mapean los valores $\vec{u}_{k}^{(0)}$ y $\vec{v}_{k}^{(0)}$ de $\vec{u}_{k}(z)$ y $\vec{v}_{k}(z)$ a el punto $z_{0}$ en sus valores a $z$. Usamos ellos para analizar el comportamiento de las soluciones en los límites IR y UV

\subsubsection{Condiciones de borde entrantes para entropía de Beckenstein-Hawking nula}

Cuando la entropía de Beckenstein-Hawking es nula, las condiciones de borde deben ser impuestas a $z_{I R} \rightarrow$ $\infty$. La matriz $\mathbf{U}(z)$ es constante allí

$$
\mathbf{U}_{I R} \equiv \mathbf{U}(\infty)=\left(\begin{array}{cccc}
-\frac{k_{y}}{g_{I R}} & \frac{\omega}{s_{I R}}-g_{I R} k_{x} & 0 & \frac{g_{I R} w_{I R}}{2} \\
-\frac{\omega}{s_{I R}}-g_{I R} k_{x} & \frac{k_{y}}{g_{R}} & \frac{g_{I R} w_{I R}}{2} & 0 \\
0 & \frac{g_{I R} w_{I R}}{2} & -\frac{k_{y}}{g_{I R}} & \frac{\omega}{s_{I R}}-g_{I R} k_{x} \\
\frac{g_{I R} w_{I R}}{2} & 0 & -\frac{\omega}{s_{I R}}-g_{I R} k_{x} & \frac{k_{y}}{g_{I R}}
\end{array}\right)
$$


donde $\left(w_{I R}, s_{I R}, g_{I R}\right)$ fue introducido en (298). Los autovalores de $\mathbf{U}_{I R}$ son dados por $\left(\kappa_{+},-\kappa_{+}, \kappa_{-},-\kappa_{-}\right)$ verificando

$$
\kappa_{ \pm}^{2}=g_{I R}^{2}\left(k_{x} \pm \frac{w_{I R}}{2}\right)^{2}+\frac{k_{y}^{2}}{g_{I R}^{2}}-\frac{\omega^{2}}{s_{I R}^{2}} .
$$

La matriz $\mathbf{U}_{I R}{ }^{2}$ es simétrica (aunque $\mathbf{U}_{I R}$ no lo es ) y entonces es diagonalizable por una matriz ortogonal $P$ tal que $P^{t} \mathbf{U}_{I R}{ }^{2} P=\operatorname{Diag}\left(\kappa_{+}{ }^{2}, \kappa_{+}{ }^{2}, \kappa_{-}{ }^{2}, \kappa_{-}{ }^{2}\right)$ y

$$
P \equiv \frac{1}{\sqrt{2}}\left(\begin{array}{cc}
1_{2 \times 2} & 1_{2 \times 2} \\
-1_{2 \times 2} & 1_{2 \times 2}
\end{array}\right) .
$$

Tomaremos $z_{0}=z_{i r} \gg 1$ y consideraremos la región $z>z_{i r} \gg 1$. Allí los operadores de evolución se escriben como

$$
\begin{aligned}
& \mathcal{C}\left(z ; z_{i r}\right) \simeq P\left(\begin{array}{cc}
\cosh \left(\kappa_{+}\left(z-z_{i r}\right)\right) 1_{2 \times 2} & 0_{2 \times 2} \\
0_{2 \times 2} & \cosh \left(\kappa_{-}\left(z-z_{i r}\right)\right) 1_{2 \times 2}
\end{array}\right) P^{t}, \\
& \mathcal{S}\left(z ; z_{i r}\right) \simeq P\left(\begin{array}{cc}
\sinh \left(\kappa_{+}\left(z-z_{i r}\right)\right) 1_{2 \times 2} & 0_{2 \times 2} \\
0_{2 \times 2} & \sinh \left(\kappa_{-}\left(z-z_{i r}\right)\right) 1_{2 \times 2}
\end{array}\right) D P^{t} \mathbf{U}_{I R},
\end{aligned}
$$

donde $D=\operatorname{Diag}\left(1 / \kappa_{+}, 1 / \kappa_{+}, 1 / \kappa_{-}, 1 / \kappa_{-}\right)$. Ahora las ecuaciónes (313) implican que en el límite de grandes valores de $z$ tenemos que

$$
\begin{aligned}
\vec{u}_{k}(z) \simeq & \frac{1}{2} P\left(\begin{array}{cc}
e^{\kappa_{+}\left(z-z_{i r}\right)} 1_{2 \times 2} & 0_{2 \times 2} \\
0_{2 \times 2} & e^{\kappa_{-}\left(z-z_{i r}\right)} 1_{2 \times 2}
\end{array}\right)\left(P^{t} \vec{u}_{k}^{(i r)}-i \tilde{D} P^{t} \mathbf{U}_{I R} \vec{v}_{k}^{(i r)}\right)+ \\
& +\frac{1}{2} P\left(\begin{array}{cc}
e^{-\kappa_{+}\left(z-z_{i r}\right)} 1_{2 \times 2} & 0_{2 \times 2} \\
0_{2 \times 2} & e^{-\kappa_{-}\left(z-z_{i r}\right)} 1_{2 \times 2}
\end{array}\right)\left(P^{t} \vec{u}_{k}^{(i r)}+i \tilde{D} P^{t} \mathbf{U}_{I R} \vec{v}_{k}^{(i r)}\right), \\
\vec{v}_{k}(z) \simeq & \frac{1}{2} P\left(\begin{array}{cc}
e^{\kappa_{+}\left(z-z_{i r}\right)} 1_{2 \times 2} & 0_{2 \times 2} \\
0_{2 \times 2} & e^{\kappa_{-}\left(z-z_{i r}\right)} 1_{2 \times 2}
\end{array}\right)\left(P^{t} \vec{v}_{k}^{(i r)}+i \tilde{D} P^{t} \mathbf{U}_{I R} \vec{u}_{k}^{(i r)}\right)+ \\
& +\frac{1}{2} P\left(\begin{array}{cc}
e^{-\kappa_{+}\left(z-z_{i r}\right)} 1_{2 \times 2} & 0_{2 \times 2} \\
0_{2 \times 2} & e^{-\kappa_{-}\left(z-z_{i r}\right)} 1_{2 \times 2}
\end{array}\right)\left(P^{t} \vec{v}_{k}^{(i r)}-i \tilde{D} P^{t} \mathbf{U}_{I R} \vec{u}_{k}^{(i r)}\right) .
\end{aligned}
$$

Vamos primero a considerar $\kappa_{ \pm}^{2}>0$, y entonces $\kappa_{ \pm} \equiv+\sqrt{\kappa_{ \pm}^{2}}$. La condición para que la solución sea bien comportada implica que los coeficientes de las exponenciales positivas deben desaparecer, teniendo la solución

$$
\begin{aligned}
& \vec{u}_{k}(z) \simeq P\left(\begin{array}{cc}
e^{-\kappa_{+}\left(z-z_{i r}\right)} 1_{2 \times 2} & 0_{2 \times 2} \\
0_{2 \times 2} & e^{-\kappa_{-}\left(z-z_{i r}\right)} 1_{2 \times 2}
\end{array}\right) P^{t} \vec{u}_{k}^{(i r)}, \\
& \vec{v}_{k}(z) \simeq P\left(\begin{array}{cc}
e^{-\kappa_{+}\left(z-z_{i r}\right)} 1_{2 \times 2} & 0_{2 \times 2} \\
0_{2 \times 2} & e^{-\kappa_{-}\left(z-z_{i r}\right)} 1_{2 \times 2}
\end{array}\right) P^{t} \vec{v}_{k}^{(i r)},
\end{aligned}
$$

donde los valores iniciales de los campos satisfacen la relación líneal

$$
\vec{u}_{k}^{(i r)}=i P \tilde{D} P^{t} \mathbf{U}_{I R} \vec{v}_{k}^{(i r)} \text {. }
$$

Un resultado similar es obtenido cuando $\kappa_{+}{ }^{2}<0 \mathrm{y} / \mathrm{o} \kappa_{-}{ }^{2}<0$ bajo la imposición de condiciones de borde entrantes (ingoing), con el reemplazo $\kappa_{ \pm} \rightarrow-i \operatorname{sign}(\omega) \sqrt{-\kappa_{ \pm}^{2}}$ en (320). Con respecto al caso $\kappa_{ \pm}^{2}=0$, sera de particular interés mas adelante, obtenemos que

$$
\begin{aligned}
& \mathcal{C}\left(z ; z_{i r}\right) \simeq 1, \\
& \mathcal{S}\left(z ; z_{i r}\right) \simeq 0,
\end{aligned}
$$

implicando los comportamientos

$$
\begin{aligned}
& \vec{u}_{k}(z) \simeq \vec{u}_{k}^{(i r)}, \\
& \vec{v}_{k}(z) \simeq \vec{v}_{k}^{(i r)} .
\end{aligned}
$$

Notemos que estas son regulares en el horizonte sin imponer ninguna relación adicional entre $\vec{u}_{k}^{(i r)}$ y $\vec{u}_{k}^{(i r)}$. 


\subsubsection{Condiciones de borde entrantes para entropía de Beckenstein-Hawking no nula}

Cuando la entropía de Beckenstein-Hawking no es nula, el horizonte se puede fijar en $z_{h}=1$, y la matriz $\mathbf{U}(z)$ no es una matriz constante allí pero presenta un polo simple

$$
\mathbf{U}(z) \stackrel{z \rightarrow 1^{-}}{\longrightarrow} \frac{-i \omega}{4 \pi T(1-z)} \mathbf{C}, \quad \mathbf{C} \equiv\left(\begin{array}{cc}
i \gamma^{0} & 0_{2 \times 2} \\
0_{2 \times 2} & i \gamma^{0}
\end{array}\right),
$$

donde $T$ es la temperatura de Hawking introducida en (302). Notemos que $\mathbf{C}$ es un imaginario puro, hermítico y satisface $\mathbf{C}^{2}=1$. Vamos a tomar ahora $\left|1-z_{i r}\right| \ll 1$, y vamos a mirar la región cerca del horizonte $z_{i r}<z \leq 1$. La evolución de los operadores (314) a primer orden se escribe entonces como

$$
\mathcal{C}\left(z ; z_{i r}\right) \simeq \cos \left(\frac{\omega}{4 \pi T} \ln \frac{1-z}{1-z_{i r}}\right) \mathbf{1}, \quad \mathcal{S}\left(z ; z_{i r}\right) \simeq i \sin \left(\frac{\omega}{4 \pi T} \ln \frac{1-z}{1-z_{i r}}\right) \mathbf{C},
$$

de (313) ellos producen la solución

$$
\begin{aligned}
& \vec{u}_{k}(z) \simeq \cos \left(\frac{\omega}{4 \pi T} \ln \frac{1-z}{1-z_{i r}}\right) \vec{u}_{k}^{(i r)}+\sin \left(\frac{\omega}{4 \pi T} \ln \frac{1-z}{1-z_{i r}}\right) \mathbf{C} \vec{v}_{k}^{(i r)} \\
& \vec{v}_{k}(z) \simeq-\sin \left(\frac{\omega}{4 \pi T} \ln \frac{1-z}{1-z_{i r}}\right) \mathbf{C} \vec{u}_{k}^{(i r)}+\cos \left(\frac{\omega}{4 \pi T} \ln \frac{1-z}{1-z_{i r}}\right) \vec{v}_{k}^{(i r)} .
\end{aligned}
$$

Las condiciones de borde entrantes en el horizonte fijan de nuevo una relacion líneal entre $\vec{u}_{k}^{(i r)}$ y $\vec{v}_{k}^{(i r)}$, como

$$
\vec{u}_{k}^{(i r)}=i \mathbf{C} \vec{v}_{k}^{(i r)} .
$$

Después de imponer esta condición cerca del horizonte la solución se escribe,

$$
\begin{aligned}
& \vec{u}_{k}(z) \simeq e^{-i \frac{\omega}{4 \pi T} \ln \frac{1-z}{1-z_{i r}}} \vec{u}_{k}^{(i r)}, \\
& \vec{v}_{k}(z) \simeq e^{-i \frac{\omega}{4 \pi T} \ln \frac{1-z}{1-z_{i r}}} \vec{v}_{k}^{(i r)} .
\end{aligned}
$$

\subsubsection{El comportamiento ultravioleta}

Vamos a tomar ahora $z_{0}=z_{U V}=0$ en las ecuaciónes (313), y considerar que la región cerca del borde AdS $z \rightarrow 0$. Allí la matriz $\mathbf{U}(z)$ va a una constante

$$
\mathbf{U}_{U V} \equiv \mathbf{U}(0)=\left(\begin{array}{cccc}
-k_{y} & \omega+\frac{\mu}{2}-k_{x} & 0 & 0 \\
-\omega-\frac{\mu}{2}-k_{x} & k_{y} & 0 & 0 \\
0 & 0 & -k_{y} & \omega-\frac{\mu}{2}-k_{x} \\
0 & 0 & -\omega+\frac{\mu}{2}-k_{x} & k_{y}
\end{array}\right)
$$

y los operadores de evolución (314) a primer orden son

$$
\mathcal{C}(z ; 0) \simeq 1, \quad \mathcal{S}(z ; 0) \simeq \mathbf{U}_{U V} z .
$$

poniendo devuelta en (313), los comportamientos a primer orden resultan ser

$$
\begin{aligned}
& \vec{u}_{k}(z) \simeq \vec{u}_{k}^{(U V)}-i z \mathbf{U}_{U V} \vec{v}_{k}^{(U V)} \\
& \vec{v}_{k}(z) \simeq \vec{v}_{k}^{(U V)}+i z \mathbf{U}_{U V} \vec{u}_{k}^{(U V)}
\end{aligned}
$$

\subsubsection{Modos normales y los conos de Dirac}

Los modos normales pueden ser definidos genéricamente como las soluciones mas normalizables regulares con energía real. En el caso de Fermiones sin masa que es el caso que consideraremos nosotros, el comportamiento cerca del borde AdS es dado por (331). El camino natural para definir los modos normales debe ser buscar soluciones donde los términos de primer orden resultan ser cero en el borde. No obstante, de acuerdo los términos de borde incluidos en nuestra acción (305), la mejor cosa que podemos hacer es definir los modos normales fermiónicos como soluciones regulares con $\vec{u}_{k}^{(U V)} \equiv \vec{u}_{k}\left(z_{U V}\right)=\overrightarrow{0}$. Debido a la suavidad/entrantes condiciones de borde en el horizonte, las componentes restantes $\vec{v}_{k}^{(U V)} \equiv \vec{v}_{k}\left(z_{U V}\right)$ son entonces completamente determinadas, y ninguna condición puede ser impuesta sobre ellas. En efecto, vamos a tomar $z_{0}=z_{i r}$ y $z=z_{U V}=0$ en $(313)$

$$
\vec{u}_{k}^{(U V)}=\mathcal{C}\left(z_{U V} ; z_{i r}\right) \vec{u}_{k}^{(i r)}-i \mathcal{S}\left(z_{U V} ; z_{i r}\right) \vec{v}_{k}^{(i r)}
$$




$$
\vec{v}_{k}^{(U V)}=i \mathcal{S}\left(z_{U V} ; z_{i r}\right) \vec{u}_{k}^{(i r)}+\mathcal{C}\left(z_{U V} ; z_{i r}\right) \vec{v}_{k}^{(i r)} .
$$

Pero como vimos en la subsección 6.6.1 y 6.6.2 una relación líneal $\vec{v}_{k}^{(i r)}=-i L \vec{u}_{k}^{(i r)}$ se sostiene donde, de acuerdo a (321) o (327), la matriz unimodular $L$ es dada por $L=U_{I R} P \tilde{D} P^{t}$ o $L=\mathbf{C}$ respectivamente. ${ }^{23}$. Por lo tanto (332) puede ser escrito como

$$
\begin{aligned}
& \vec{u}_{k}^{(U V)}=\left(\mathcal{C}\left(z_{U V} ; z_{i r}\right)-\mathcal{S}\left(z_{U V} ; z_{i r}\right) L\right) \vec{u}_{k}^{(i r)}, \\
& \vec{v}_{k}^{(U V)}=i\left(\mathcal{S}\left(z_{U V} ; z_{i r}\right)-\mathcal{C}\left(z_{U V} ; z_{i r}\right) L\right) \vec{u}_{k}^{(i r)},
\end{aligned}
$$

la cual muestra que para un momento general $\vec{v}_{k}^{(U V)}$ es determinado una vez que $\vec{u}_{k}^{(U V)}$ es dado. No obstante, para modos normales fijamos la condición de borde particular $\vec{u}_{k}^{(U V)}=0$, y entonces existirá una solución no trivial si

$$
C\left(\omega, k_{x}, k_{y}\right) \equiv \operatorname{det}\left(\mathcal{C}\left(z_{U V} ; z_{i r}\right)-\mathcal{S}\left(z_{U V} ; z_{i r}\right) L\right)=0 .
$$

Esta ecuación define una restricción en el espacio de momento para cualquier valor de la frecuencia, donde los modos normales viven. Ahora vamos a dar algún argumento eurístico para analizar algún límite de esta ecuación:

- En primer lugar vamos a considerar partículas altamente energéticas. Ya que ellas sondean el espacio cerca del borde, son prácticamente ciegas a la región IR, entonces podemos usar (330) como una aproximación para sus operadores de evolución, i.e $\mathcal{C}\left(0 ; z_{i r}\right) \simeq \mathcal{C}\left(z_{i r} ; 0\right) \simeq 1, \mathcal{S}\left(0 ; z_{i r}\right) \simeq-\mathcal{S}\left(z_{i r} ; 0\right) \simeq$ $-\mathbf{U}_{U V} z_{i r}$. En tal caso, la ecuación (334) se escribe

$$
C\left(\omega, k_{x}, k_{y}\right) \simeq \operatorname{det}\left(1+z_{i r} \mathbf{U}_{U V} L\right) \simeq z_{i r}{ }^{4} \operatorname{det}\left(\mathbf{U}_{U V}\right)=0
$$

Por lo tanto en este límite los modos normales tienen su momento y frecuencia tal que los autovalores de $\mathbf{U}_{U V}$, son dados por $\left(\lambda_{+},-\lambda_{+}, \lambda_{-},-\lambda_{-}\right)$, con

$$
\lambda_{ \pm}^{2} \equiv k_{x}^{2}+k_{y}^{2}-\left(\omega \pm \frac{\mu}{2}\right)^{2}
$$

desaparece. Las ecuaciónes $\lambda_{ \pm}^{2}=0$ pueden entonces ser tomadas como una aproximación de la restricción $C\left(\omega, k_{x}, k_{y}\right)=0$ para un gran valor de $|\omega|$. Ellas definen los conos de Dirac UV $C_{ \pm}^{U V}$ [14], cuyos vértices están localizados a $\left(\omega, k_{x}, k_{y}\right)=(\mp \mu / 2,0,0)$, y estos satisfacen $C_{+}^{U V} \cap C_{-}^{U V^{ \pm}}=$ $\left\{\left(0, k_{x}, k_{y}\right): k_{x}^{2}+k_{y}^{2}=\mu^{2} / 4\right\}$. Notemos que ellos presentan simetría rotacional en el plano de los momentos, indicando que tal simetría es preservada en el UV.

- Por otro lado, si consideramos los modos de baja energía, ellos sondean la región de grandes valores de $z$ siendo esencialmente ciegas a la geometría en el UV. Entonces para $\kappa_{ \pm}^{2} \neq 0$ podemos usar (318) como una aproximación para sus operadores evolución. La restricción (334) esta vez se convierte en

$$
C\left(\omega, k_{x}, k_{y}\right) \simeq e^{\left(\kappa_{+}+\kappa_{-}\right) z_{i r}}=0
$$

la cual no puede ser verificada para cualquier elección de momento y frecuencia. Entonces nos quedamos con el caso $\kappa_{ \pm}^{2}=0$, donde

$$
\kappa_{ \pm}^{2}=g_{I R}^{2}\left(k_{x} \pm \frac{w_{I R}}{2}\right)^{2}+\frac{k_{y}^{2}}{g_{I R}^{2}}-\frac{\omega^{2}}{s_{I R}^{2}} .
$$

entonces la restricción $C\left(\omega, k_{x}, k_{y}\right)=0$ puede ser aproximada como $\kappa_{ \pm}^{2}=0$. Esto define los dos conos de Dirac en el IR $C_{ \pm}^{I R}[14]$. Sus vértices están localizados a $\left(\omega, k_{x}, k_{y}\right)=\left(0, \mp w_{I R} / 2,0\right)$, y ellos satisfacen $C_{+}^{I R} \bigcap C_{-}^{I R}=\left\{\left(\omega, 0, k_{y}\right): \omega^{2}=s_{I R}^{2}\left(g_{I R}^{2} w_{I R}^{2} / 4+k_{y}^{2} / g_{I R}^{2}\right)\right\}$. Notemos que la simetría rotacional en el plano de los momentos esta rota en el IR.

\subsection{Operadores fermiónicos en la QFT}

Desde el punto de vista de la correspondencia AdS/CFT, los campos espinoriales definidos arriba, tienen un operador espinorial dual $\mathcal{O}(x)$ en el borde, el cual transforma como un doblete en la representación de $S U(2)$. Las funciónes de correlación de tal operador pueden ser computadas derivando la acción on-shell con respecto a la fuente identificada rudamente con el valor en el borde que toma el campo espinorial. En esta subsección presentaremos los cómputos de la función de correlación retardada fermiónica siguiendo de cerca la referencia [14].

\footnotetext{
${ }^{23}$ Los modos normales son estrictamente definidos a $T=0$, entonces nos restringiremos a este caso por ahora.
} 
Cuando evaluamos la acción (305) sobre una solución de las ecuaciónes de movimiento solo los términos de borde contribuyen. En términos de la notación vectorial (310) toma la forma

$$
S_{\text {on-shell }}^{(f e r)}=-\int \frac{d^{3} k}{(2 \pi)^{3}} \vec{u}_{k}^{(U V) \dagger} \mathbf{C} \vec{v}_{k}^{(U V)}
$$

No obstante, como notamos antes, una relación existe para un valor de momento general entre los valores de borde de los campos; de (333) obtenemos

$$
\begin{aligned}
\vec{v}_{k}^{(U V)} & =\mathbf{M}_{k} \vec{u}_{k}^{(U V)} \\
\mathbf{M}_{k} & \equiv i\left(\mathcal{S}\left(z_{U V} ; z_{i r}\right)-\mathcal{C}\left(z_{U V} ; z_{i r}\right) L\right)\left(\mathcal{C}\left(z_{U V} ; z_{i r}\right)-\mathcal{S}\left(z_{U V} ; z_{i r}\right) L\right)^{-1}
\end{aligned}
$$

entonces la acción on-shell es

$$
S_{\text {on-shell }}^{(f e r)}=-\int \frac{d^{3} k}{(2 \pi)^{3}} \vec{u}_{k}^{(U V) \dagger} \mathbf{C ~} \mathbf{M}_{k} \vec{u}_{k}^{(U V)} .
$$

En nuestro notación de 4-upla, las fuentes $\vec{u}_{k}^{(U V)}$ se acoplan a 4- uplas de operadores fermiónicos $\overrightarrow{\mathcal{O}}(x)=$ $\left(\mathcal{O}^{I}(x), I=1, \ldots, 4\right)$ en la acción de la teoría del borde, por un término de la forma $\delta S=\vec{u}_{k}^{U V \dagger} \mathbf{C} \overrightarrow{\mathcal{O}}+h . c .$. La correspondencia AdS/CFT establece que la función de Green retardada conectada de ellos en el espacio de momentos puede ser computada por medio de la receta

$$
-\left.i\left\langle\overrightarrow{\tilde{O}}(k) \overrightarrow{\tilde{O}}^{\dagger}(0)\right\rangle_{c}\right|_{\text {ret }} \equiv(2 \pi)^{3} \delta^{3}(k) \mathbf{G}_{R}(k)=\mathbf{C} \frac{\delta^{2} S_{\text {on-shell }}^{(f e r)}}{\delta \vec{u}_{k}^{(U V)_{\dagger}} \delta \vec{u}_{0}^{(U V)}} \mathbf{C} .
$$

De aquí, usando la ecuación (341),podemos escribir la función de correlación

$$
\mathbf{G}_{R}(k)=\mathbf{M}_{k} \mathbf{C} .
$$

Desde el punto de vista de la teoría fermiónica, el observable relevante para analizar la dinámica de fermiones es la función espectral $\rho(k)$, definida como

$$
\rho(k) \equiv-\operatorname{Im} \operatorname{Tr} \mathbf{G}_{R}(k)=-\operatorname{Im} \operatorname{Tr}\left(\mathbf{M}_{k} \mathbf{C}\right) .
$$

Es una cantidad siempre positiva, un hecho que desde el punto de vista holográfico puede ser mostrado ver [14] en el caso de $T=0$; nosotros revisamos y extendemos el análisis para temperatura finita en el apéndice B1. Para teorías invariantes frente a traslaciones débilmente acopladas, una representación vía KähllenLehmann para $G_{R}(k)$ implica que $\rho(k)$ genéricamente presenta deltas (picos) como función de la frecuencia, sobre los estados de cuasi-partículas con una relación de dispersión bien definida, ver por ejemplo [86].

Resulta de particular relevancia el valor de la función espectral para frecuencia cero $\rho\left(0, k_{x}, k_{y}\right)$, cuyos picos serán etiquetados $\left(k_{x}^{*}, k_{y}^{*}\right)$. A temperatura nula, estos picos corresponden al momento de Fermi $\left(k_{x}^{F}, k_{y}^{F}\right)$, y su localización en el espacio de momentos determinan la superficie de Fermi. Notemos que los modos normales a $\omega=0$ se sientan sobre tal superficie, ya que la matriz $\mathbf{M}_{k}$ es singular en ellos.

\subsection{Resultados numéricos}

Realizamos un extensivo estudio numérico de todo el espacio de parámetros, para analizar como el acoplamiento gravitacional afecta la función espectral cuando la temperatura es modificada. Los resultados están contenidos en las figuras mostradas en esta sección. Gráficamos la función espectral como función de $\left(k_{x}, k_{y}\right)$ para diferentes valores de $T$ para un valor fijo de $\omega$ y $\lambda$, y como una función de $\omega$ para valores fijos de $\left(k_{x}, k_{y}\right)$, para diferentes valores de $\lambda$ y $T$.

En la figura 42 presentamos los gráficos de la función espectral como función de $\omega$ para valores fijos de $\left(k_{x}, k_{y}\right)$ y valores de $\lambda<\lambda_{c}$, y a bajas temperaturas $T<T_{c}$. Es visto que la intersección con los conos de luz en el IR son suavizados a temperatura no nula con respecto al caso $T=0$, como puede ser visto comparando la figura 8 de la referencia [14]. La región para pequeños valores de $\omega$, la función espectral oscila dando lugar a un único pico y luego tiende a un valor constante para $\omega=0$.

En la figura 43 damos la función espectral en la fase normal y superconductora a $T<T_{c}$ para diferentes valores de momento. Es observado la ausencia de paridad en $\omega$ en la fase normal en acuerdo con [67], y la presencia de tal simetría en la fase superconductora, resulta observada para el caso $T=0$ en [14].

Las figuras 44 a 52 muestran la evolución de la función espectral como función de la temperatura. Cada figura se corresponde a un valor fijo de $\lambda$ y $\omega$. En todos los casos cuando la temperatura decrece de la 


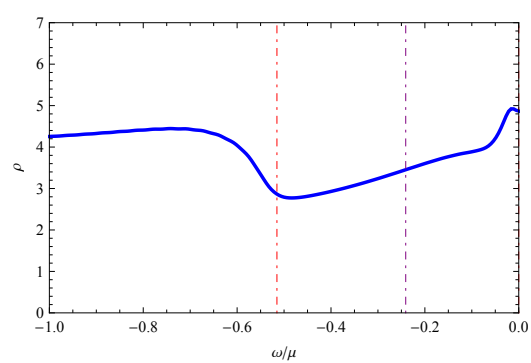

(a) $\left(k_{x}, k_{y}\right)=\left(k_{*}, 0\right) \approx(0.27,0) \mu$

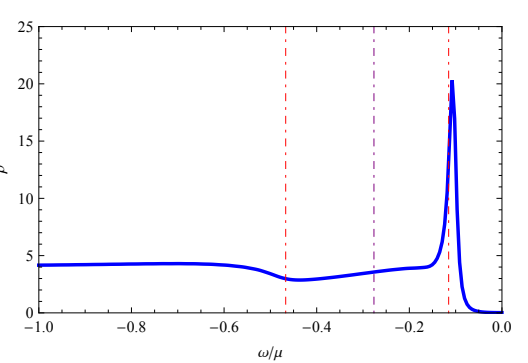

(b) $\left(k_{x}, k_{y}\right)=(0.2,0.1) \mu$

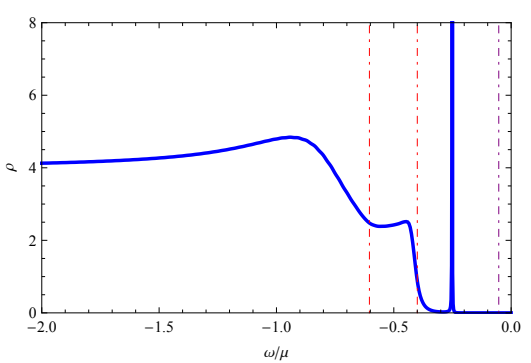

(c) $\left(k_{x}, k_{y}\right)=(0.2,0.4) \mu$

Figura 42: La función espectral como función de $\omega$ para diferentes $\left(k_{x}, k_{y}\right)$, y a baja temperatura $T=0.20 T_{c}$ y $\lambda=1 /(8 \sqrt{2})$. La líneas verticales punteadas representan la posición de los conos de Dirac IR (en rojo) y UV (en violeta).

temperatura crítica, la simetría esférica rápidamente se pierde y el máximo local se vuelve mas agudo y se aproximan al lugar de los modos normales. Notemos que, aunque por razones ilustrativas nosotros incluimos las localizaciones de los modos normales en todos los gráficos, como mencionamos en el pie de página 23 en la sección 6.6.4, estos modos son estrictamente definidos solo a $T=0$. Comparando la localización del máximo en, por ejemplo, figura 45 subfigura (b) con figura 44 subfigura (f), o figura 46 subfigura (b) con figura 45 subfigura (f), vemos que para altas temperaturas ellos se aproximan a los lugares de los modos normales correspondientes a altas frecuencias. Esto es debido al hecho de que la temperatura y frecuencia son medidas en unidades del potencial químico $\mu$, por lo que la cantidad $T / \mu$ puede ser interpretada como el conjunto formado por las figuras 44 a 46, figuras 47 a 49, y figuras 50 a 52 respectivamente corresponden a tres valores fijos de $\lambda$, uno mas pequeño, intermedio y grande comparado con $\lambda_{c}$. Cada figura en el marco, muestra los gráficos de la función espectral para diferentes valores de temperatura para un valor fijo de $\omega$. El valor de $\omega$ decrece de figura a figura dentro de cada marco. Es evidente comparando las figuras correspondientes a la misma temperatura dentro de un único cuadro, que la región para la cual la función espectral es apreciable en el espacio de momentos se vuelve mas pequeña conforme $\omega$ decrece para un valor fijo de $\lambda$. Comparando diferentes conjuntos de figuras vemos que la dependencia en la temperatura y frecuencia de la función espectral es mas suave para $\lambda>\lambda_{c}$ que en los casos $\lambda \leq \lambda_{c}$. Notemos también que incluso si la simetría esférica esta ausente, parece ser recuperada cuando la frecuencia es aumentada. Para $\lambda>\lambda_{c}$ y altas frecuencias, de la figura 50, los modos normales no existen. Esto ultimo esta en acuerdo con la ausencia de una región entre los conos de Dirac IR y UV. En su lugar en la figura 52 dos pequeñas regiónes abiertas y desconectadas están presentes, donde los modos normales aparecen.

Las figuras 53 a 55 corresponde a tres diferentes valores de $\lambda$, mas pequeño, intermedio y mas grande comparados con $\lambda_{c}$. En el lado izquierdo la superficie espectral $\omega=0$ es mostrada para diferentes temperaturas. En el lado derecho, la función espectral como función de la frecuencia es mostrada para varias temperaturas, para tres momentos diferentes. De estas funciónes, las subfiguras (1) son graficadas para el valor de momento $\left(k_{x}, k_{y}\right)_{*}=\left(k_{*}, 0\right)$ que corresponde a uno de los dos máximos de la superficie espectral a cada temperatura, y se mueve al valor de momento de Fermi conforme la temperatura tiende a cero. Definiendo el momento de Fermi $k_{F}$ como la localización de uno de los máximos cuando la temperatura es nula $T=0$, la superficie de Fermi consiste de dos puntos $\pm\left(k_{x}^{F}, k_{y}^{F}\right)=\left( \pm k_{F}, 0\right)$. Es observado que los picos en la función espectral como una función de $\omega$ en las subfiguras (2) (3) se mueve a la izquierda y se vuelve muy agudo conforme la temperatura decrece. Un pico puntiagudo similar surge en la subfigura (1), aunque es mas difícil de apreciar con nuestros métodos numéricos. Esto corresponde a la aparición de los modos normales a $T=0$. De forma interesante en la subfigura (1) los picos también se vuelven agudos conforme la temperatura se aproxima a $T_{c}$

Cuando la temperatura tiende a cero, la densidad espectral en 53 a 55 subfigura (1) se aproxima a un continuo con una especia de pico en forma de delta a $\omega=0$. Esto nos permite interpretar la inversa del peso del pico a $\omega=0$ como un rudo estimador de su ancho, i.e. de la vida media de la cuasi-partícula. En la figura 56 mostramos el valor de la densidad espectral a $\omega=0$ como función de la temperatura. Para temperaturas suficientemente bajas, tal valor se incrementa conforme la temperatura decrece, indicando el esperado y agudo pico de una cuasi-partícula. Algo interesante es que, un pico agudo sucede no solo cuando la temperatura se aproxima a cero, sino que también cuando nos aproximamos a $T_{c}$, donde se une perfectamente con los valores de la fase normal. 


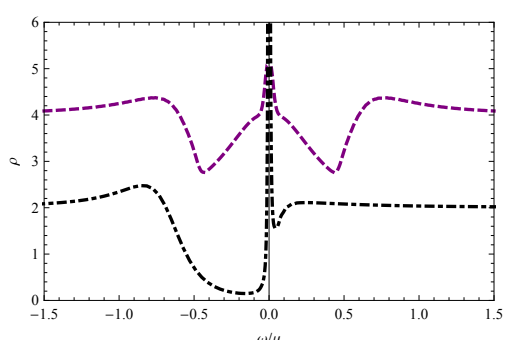

(a) $\left(k_{x}, k_{y}\right)=\left(k_{*}, 0\right)$

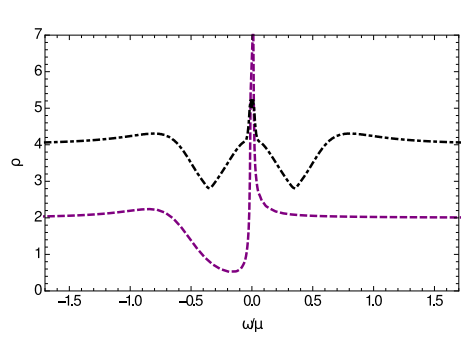

(d) $\left(k_{x}, k_{y}\right)=\left(k_{*}, 0\right)$

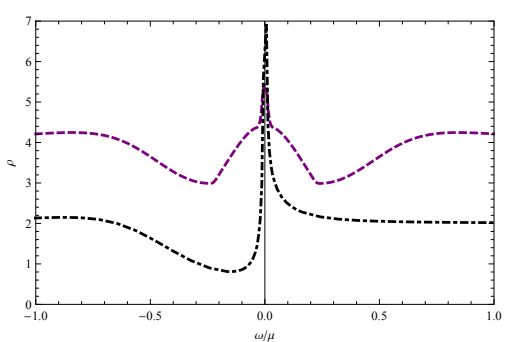

(g) $\left(k_{x}, k_{y}\right)=\left(k_{*}, 0\right)$

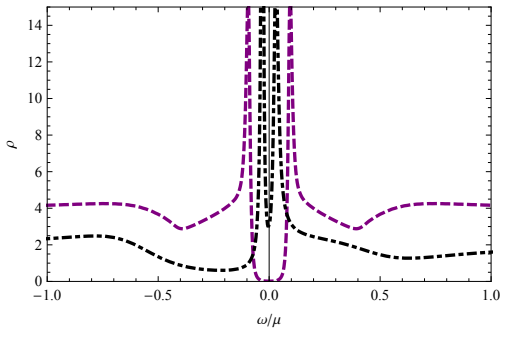

(b) $\left(k_{x}, k_{y}\right)=(0.2,0.1) \mu$

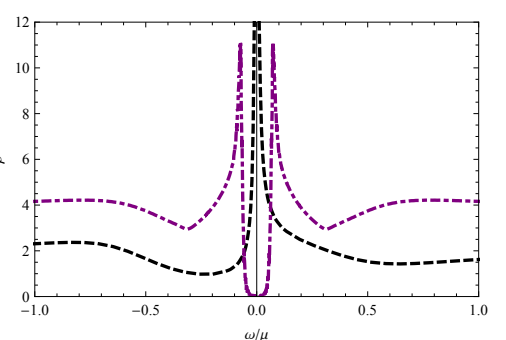

(e) $\left(k_{x}, k_{y}\right)=(0.2,0.1) \mu$

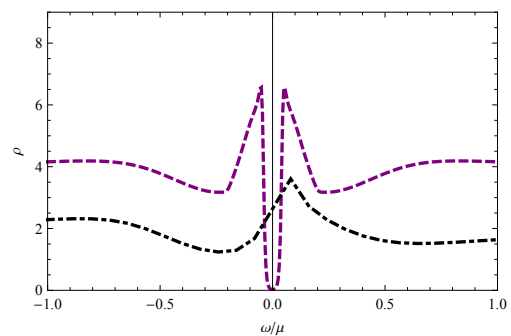

(h) $\left(k_{x}, k_{y}\right)=(0.2,0.1) \mu$

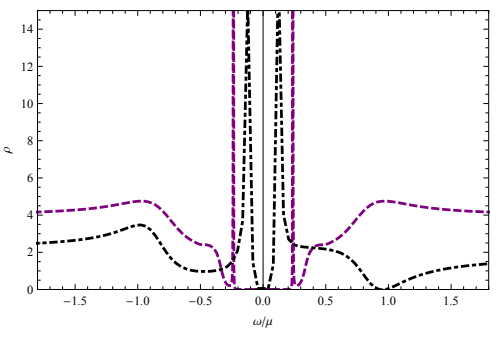

(c) $\left(k_{x}, k_{y}\right)=(0.2,0.4) \mu$

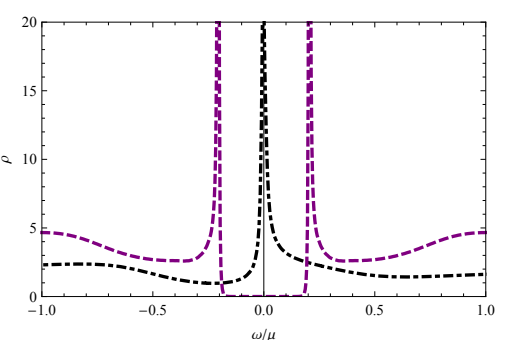

(f) $\left(k_{x}, k_{y}\right)=(0.2,0.4) \mu$

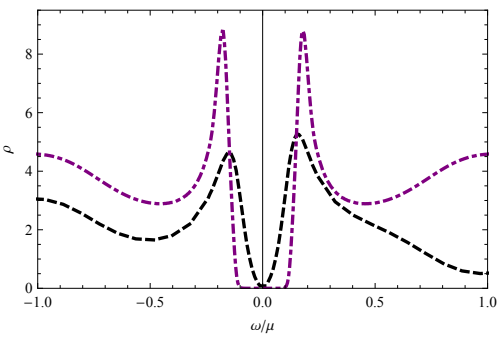

(i) $\left(k_{x}, k_{y}\right)=(0.2,0.4) \mu$

Figura 43: La función espectral como función de $\omega$ en la fase normal (negra) y la fase superconductora (violeta), a un valor fijo de $\left(k_{x}, k_{y}\right)$, para tres diferentes valores de temperatura y acoplamientos: (a) (b) (c) $T=0.20 T_{c}, \lambda=0.40<\lambda_{c} ;$ (d) (e) (f) $T=0.28 T_{c}, \lambda=0.62=\lambda_{c} ;(\mathrm{g})$ (h) (i) $T=0.40 T_{c}, \lambda=0.80>\lambda_{c}$. 


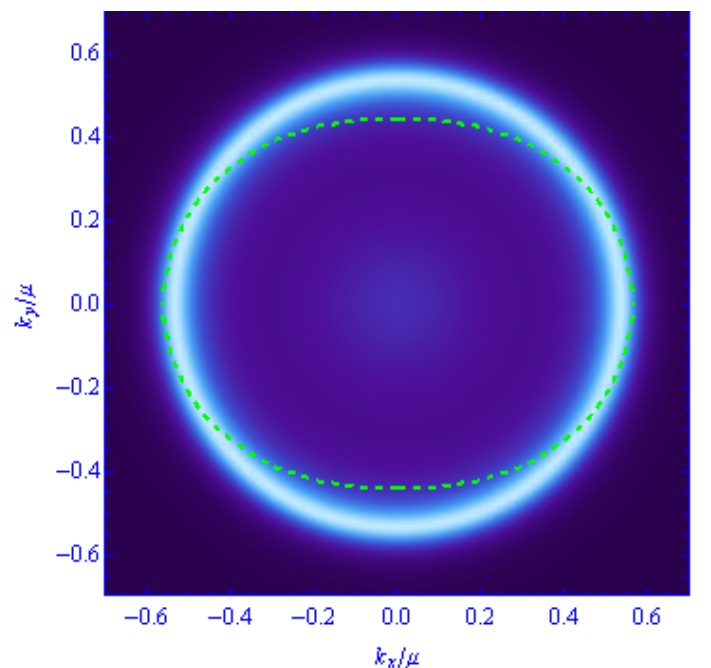

(a) $T=T_{c}$

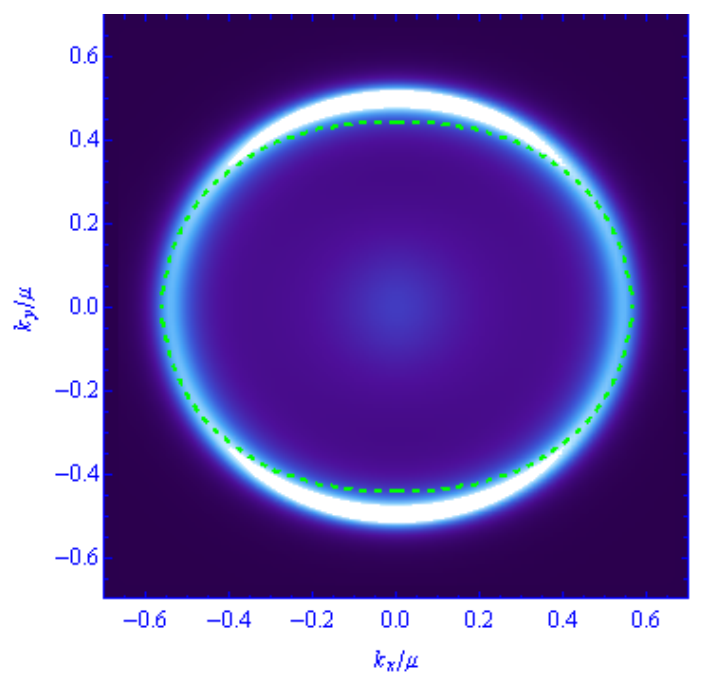

(c) $T=0.60 T_{c}$

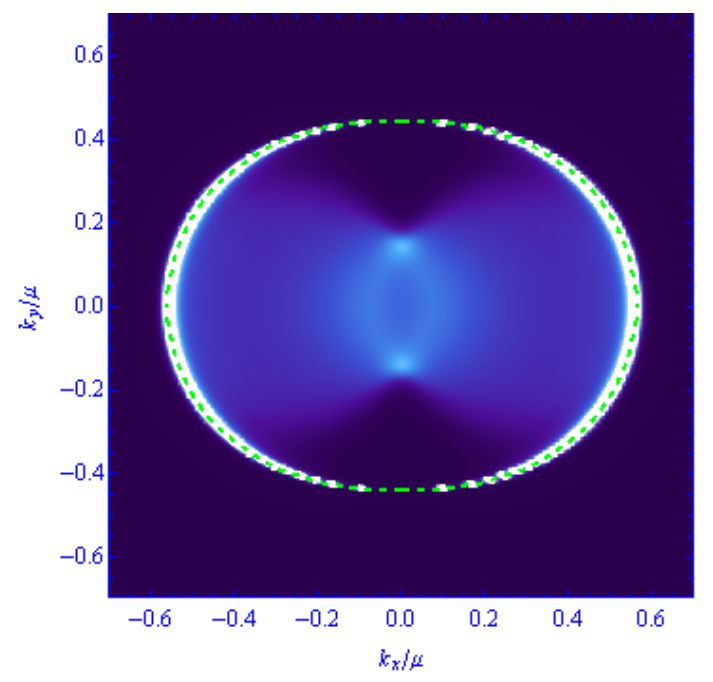

(e) $T=0.20 T_{c}$

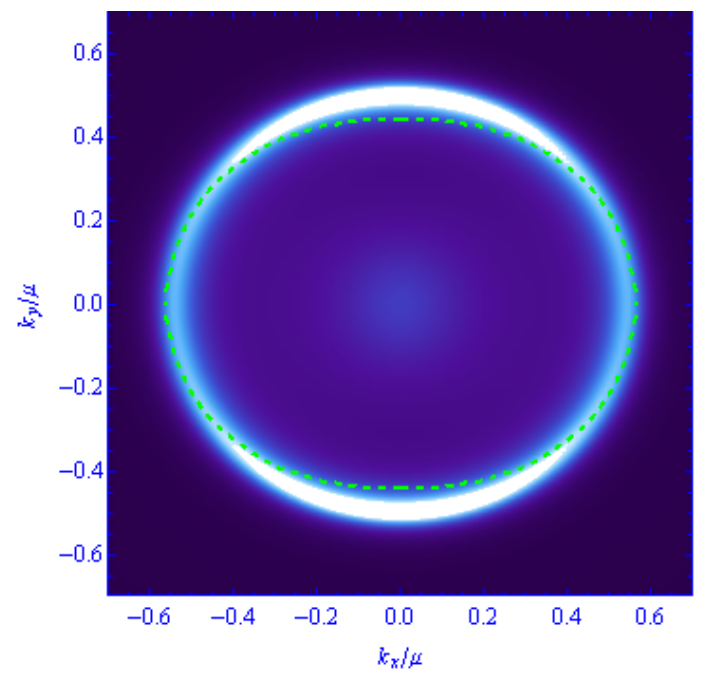

(b) $T=0.85 T_{c}$

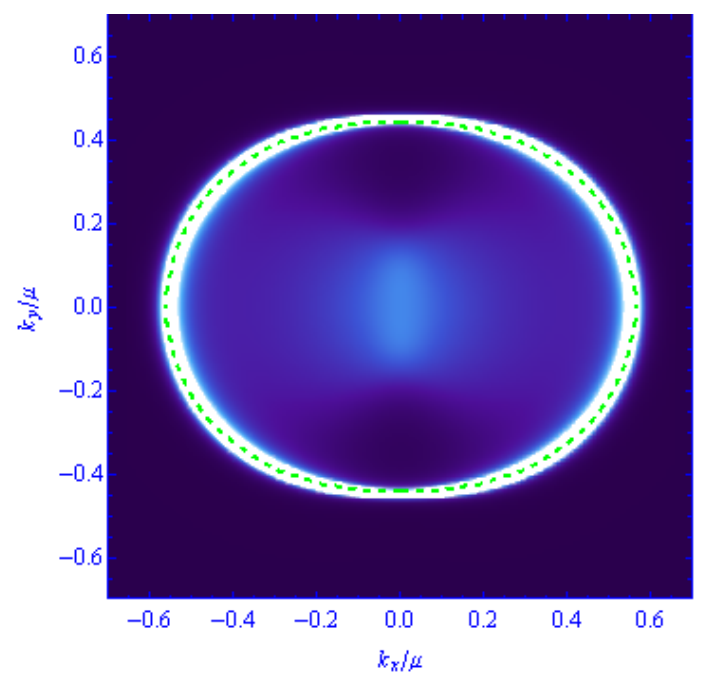

(d) $T=0.43 T_{c}$

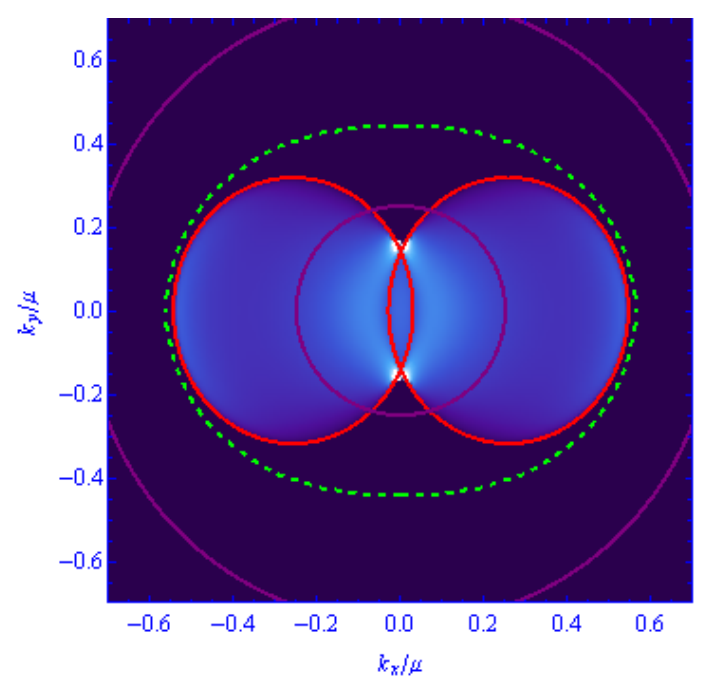

(f) $T=0$

Figura 44: La función espectral para diferentes temperaturas y para un valor fijo de $\lambda=0.40<\lambda_{c}$, $\omega=0.25 \mu$. Se observa la presencia de simetría esférica a $T=T_{c}$. Las líneas punteadas indican el lugar de los modos normales. Los círculos violetas y rojos representa los conos de Dirac UV y IR respectivamente. 


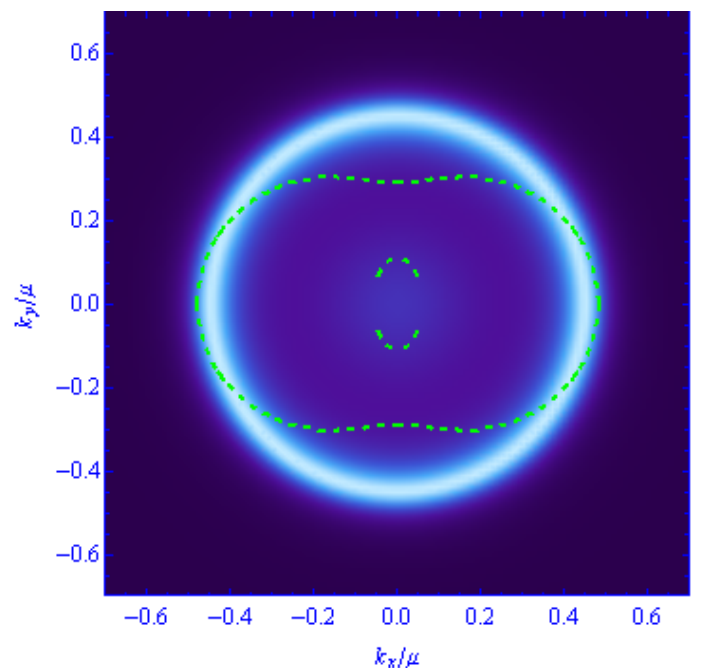

(a) $T=T_{c}$

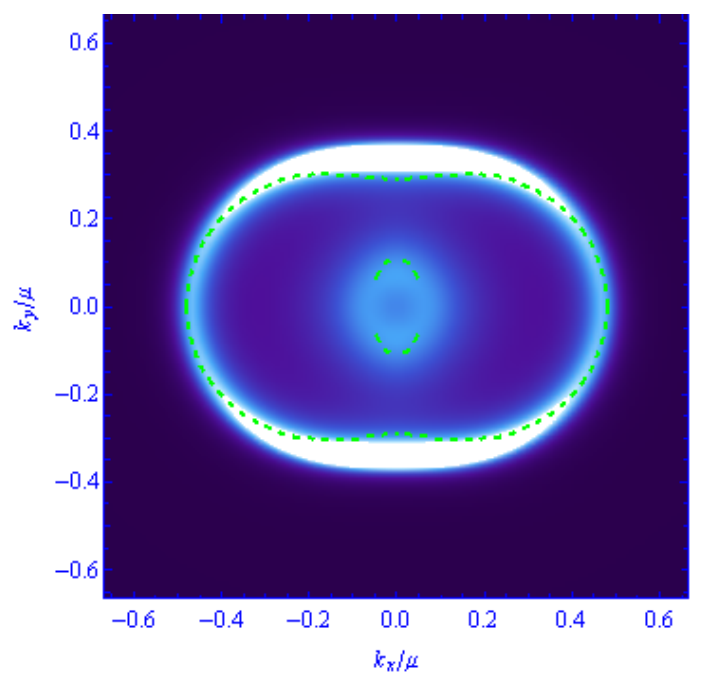

(c) $T=0.60 T_{c}$

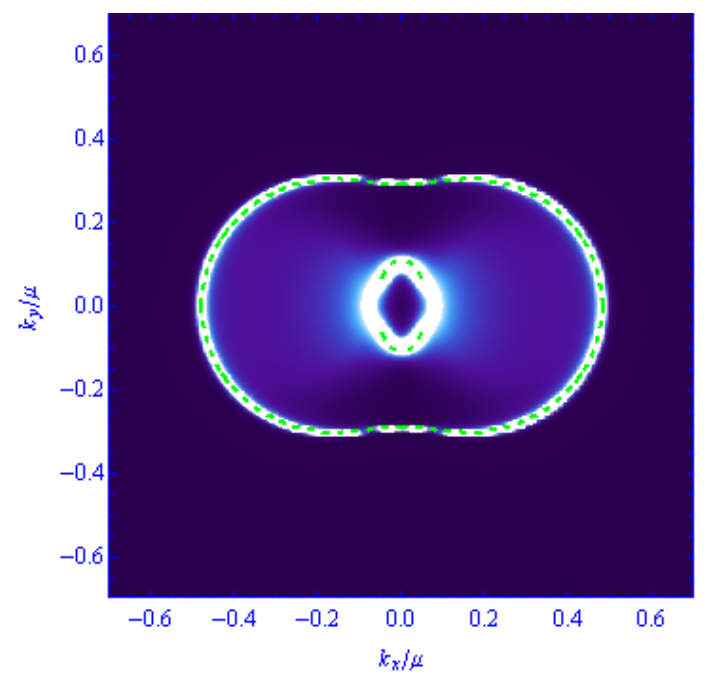

(e) $T=0.20 T_{c}$

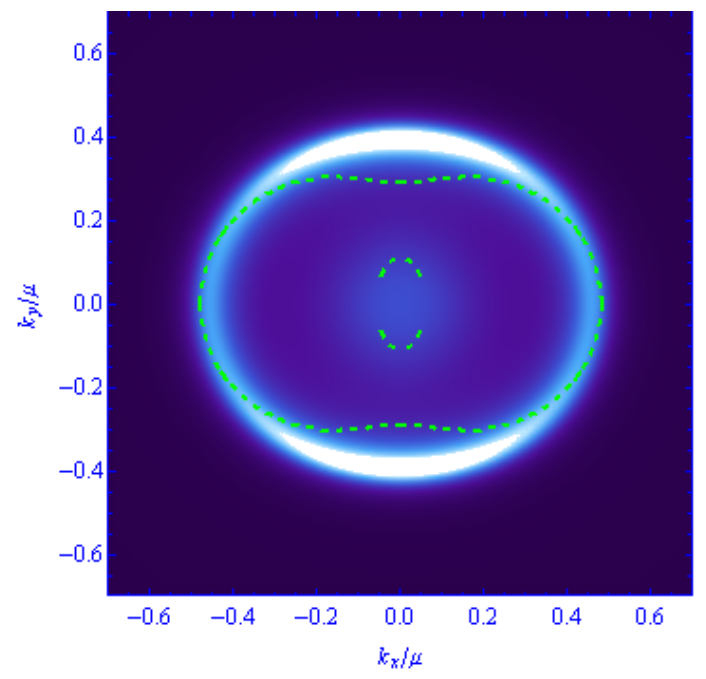

(b) $T=0.85 T_{c}$

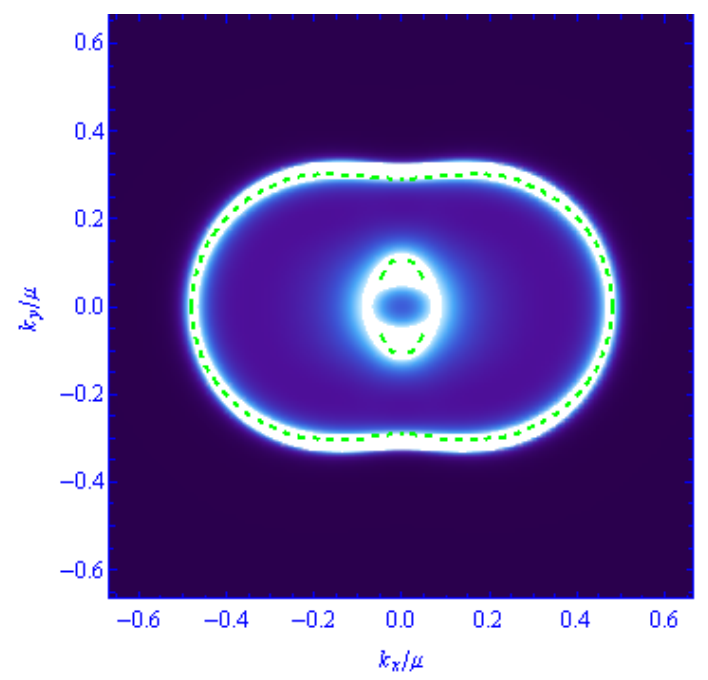

(d) $T=0.43 T_{c}$

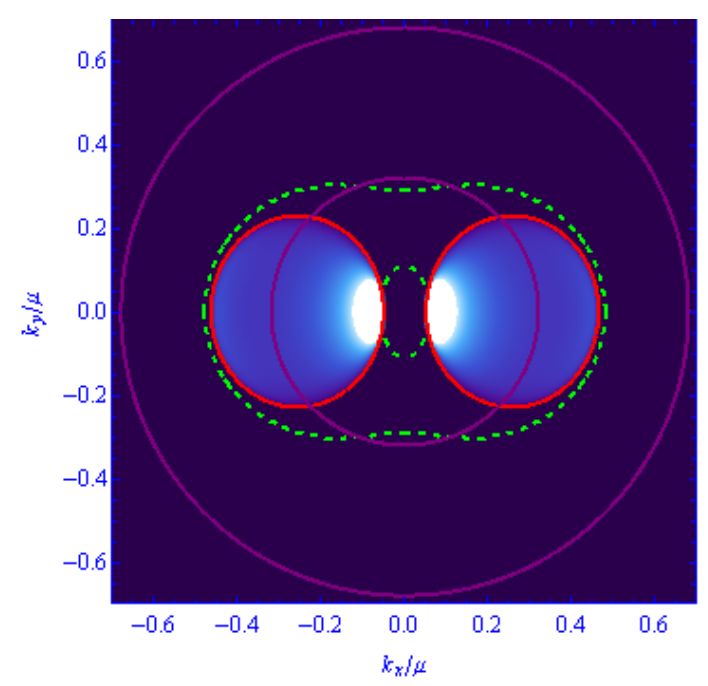

(f) $T=0$

Figura 45: La función espectral para diferentes temperaturas y para un valor fijo de $\lambda=0.40<\lambda_{c}$, $\omega=0.18 \mu$. Se observa la presencia de simetría esférica a $T=T_{c}$. Las líneas punteadas indican el lugar de los modos normales. Los círculos violetas y rojos representa los conos de Dirac UV y IR respectivamente. 


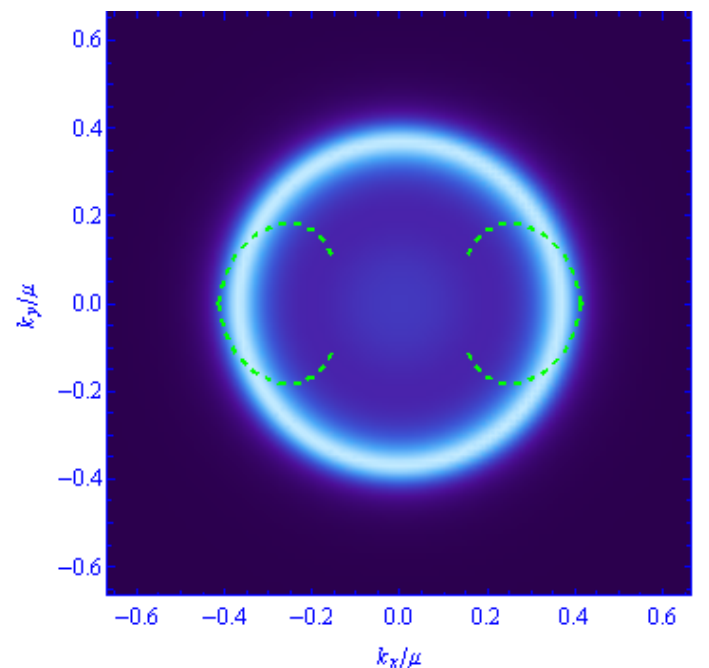

(a) $T=T_{c}$

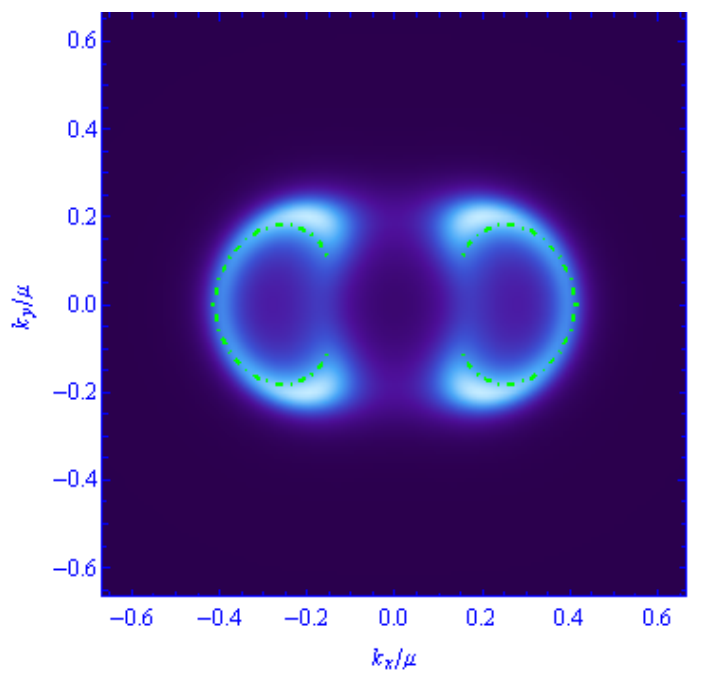

(c) $T=0.60 T_{c}$

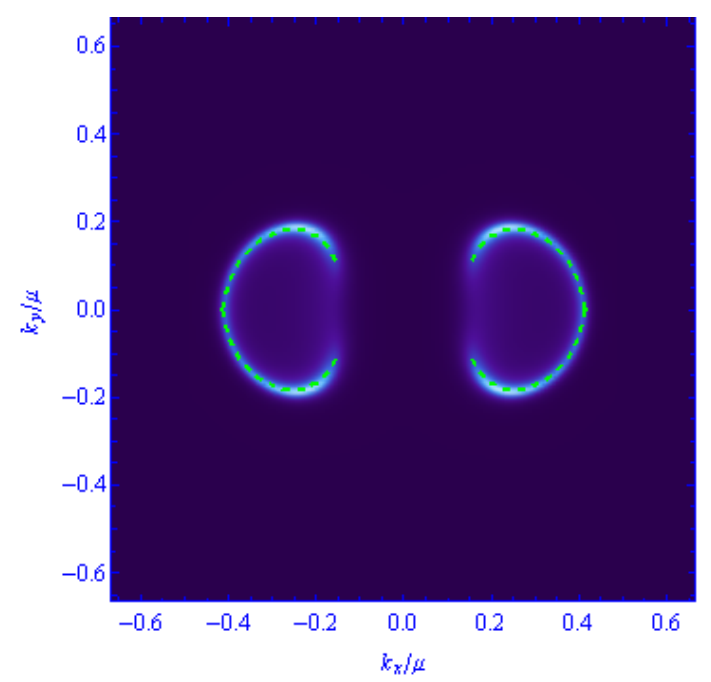

(e) $T=0.20 T_{c}$

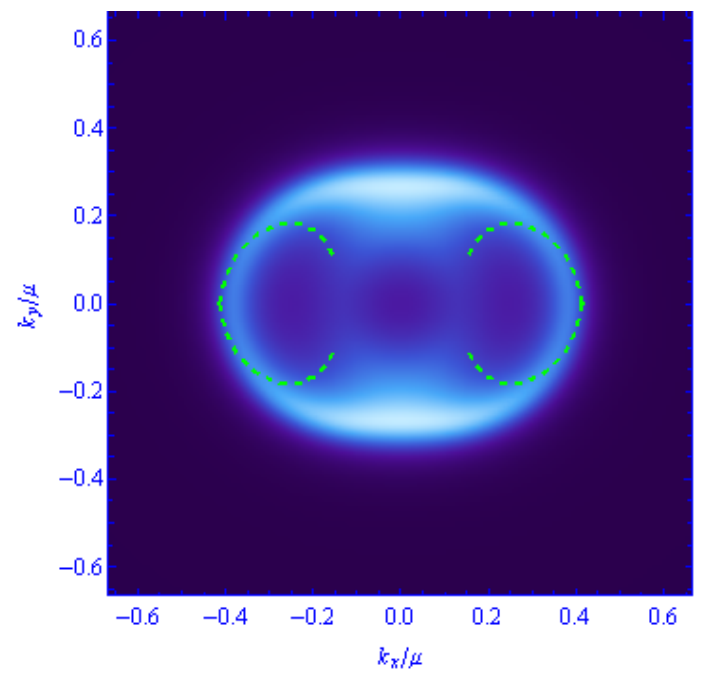

(b) $T=0.85 T_{c}$

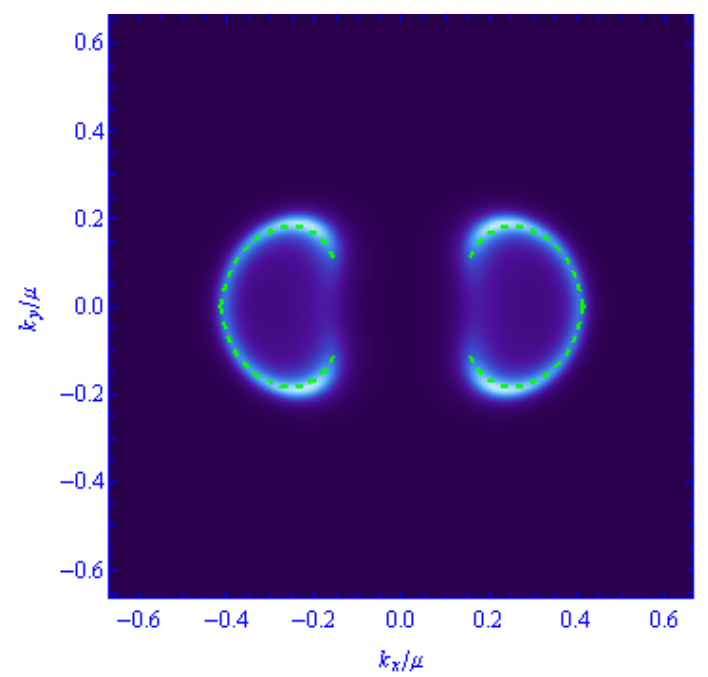

(d) $T=0.43 T_{c}$

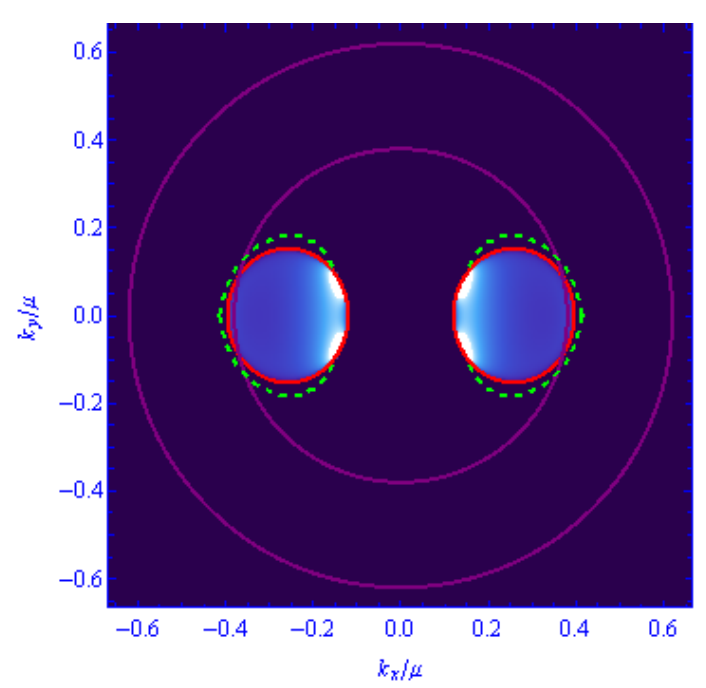

(f) $T=0$

Figura 46: La función espectral para diferentes temperaturas y para un valor fijo de $\lambda=0.40<\lambda_{c}$, $\omega=0.12 \mu$. Se observa la presencia de simetría esférica a $T=T_{c}$. Las líneas punteadas indican el lugar de los modos normales. Los círculos violetas y rojos representa los conos de Dirac UV y IR respectivamente. 


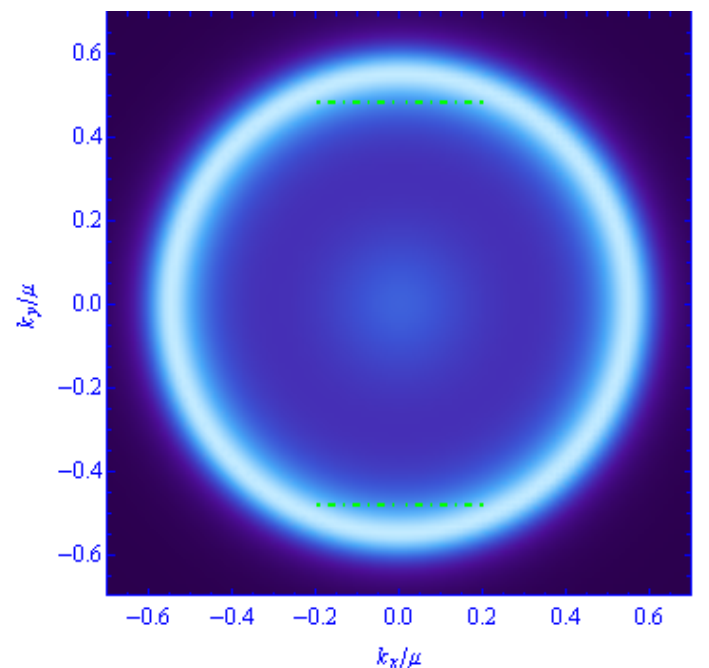

(a) $T=T_{c}$

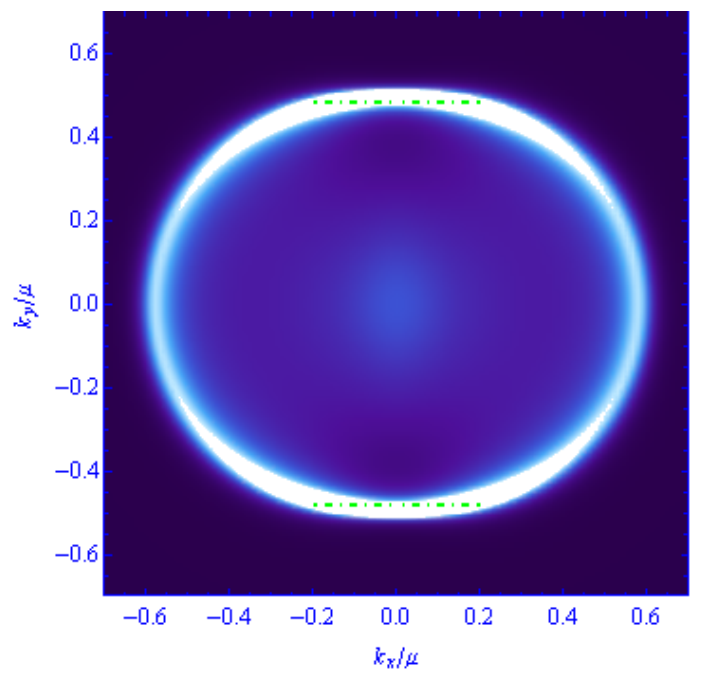

(c) $T=0.60 T_{c}$

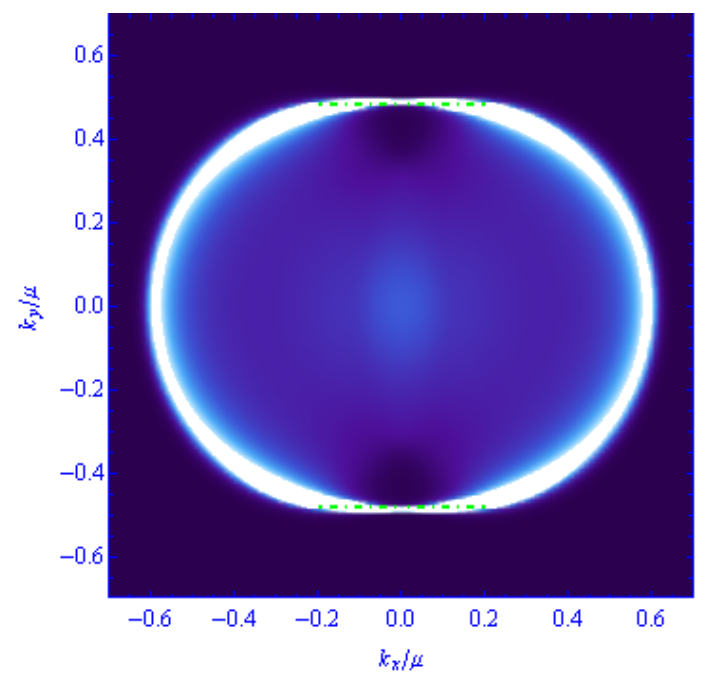

(e) $T=0.28 T_{c}$

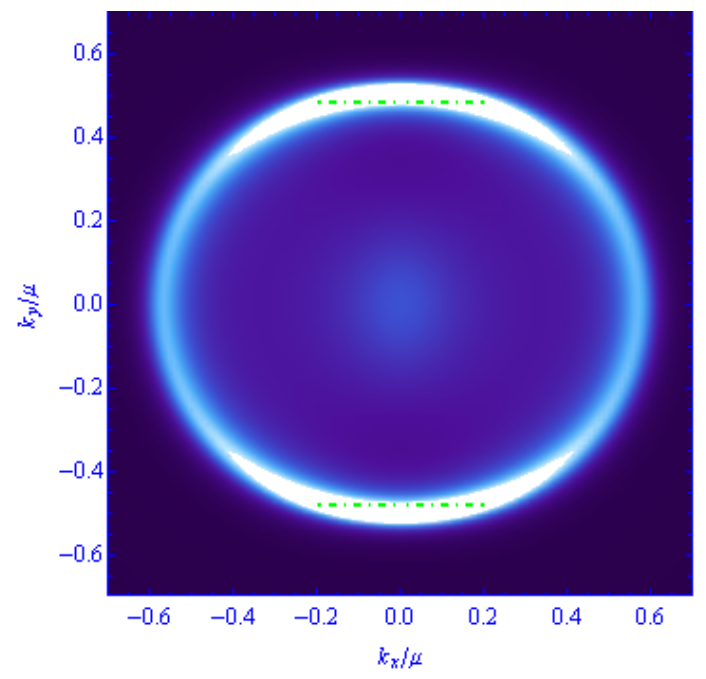

(b) $T=0.79 T_{c}$

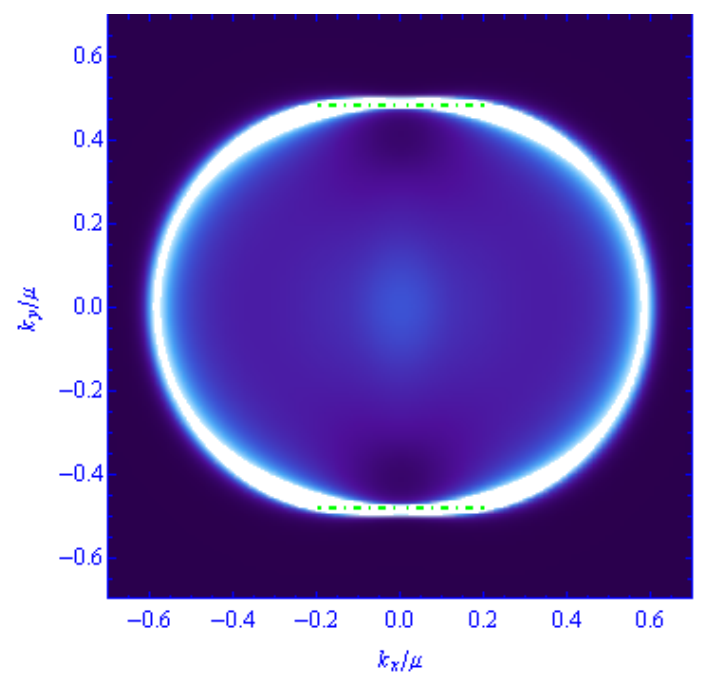

(d) $T=0.38 T_{c}$

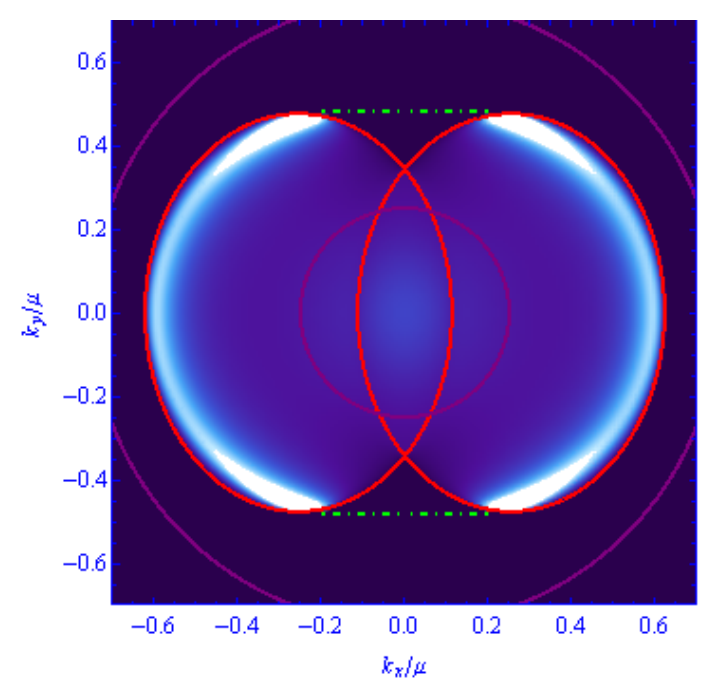

(f) $T=0$

Figura 47: La función espectral para diferentes temperaturas y para un valor fijo de $\lambda=0.62=\lambda_{c}, \omega=0.25 \mu$. Se observa la presencia de simetría esférica a $T=T_{c}$. Las líneas punteadas indican el lugar de los modos normales. Los círculos violetas y rojos representa los conos de Dirac UV y IR respectivamente. 


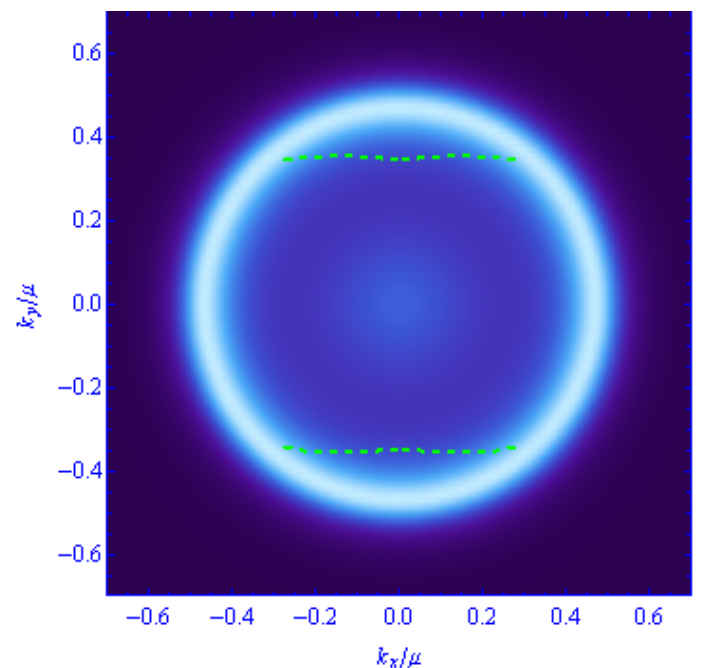

(a) $T=T_{c}$

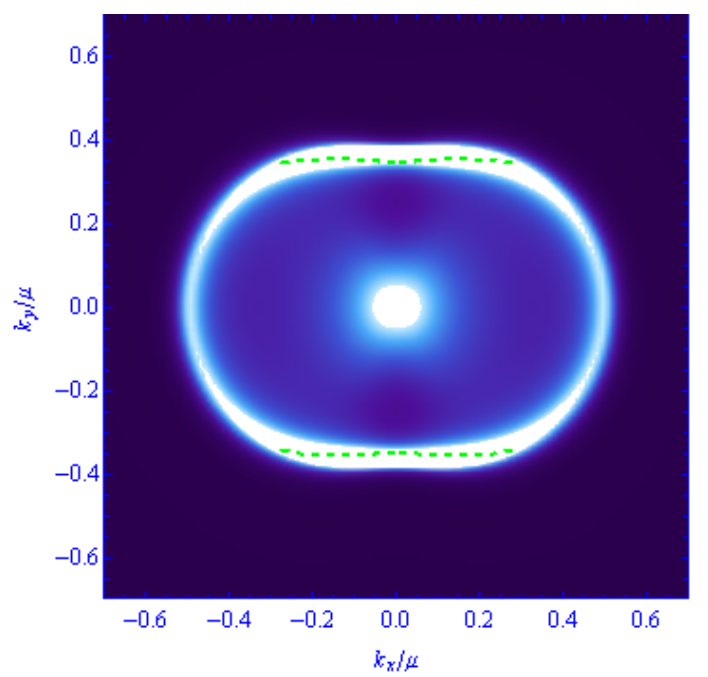

(c) $T=0.60 T_{c}$

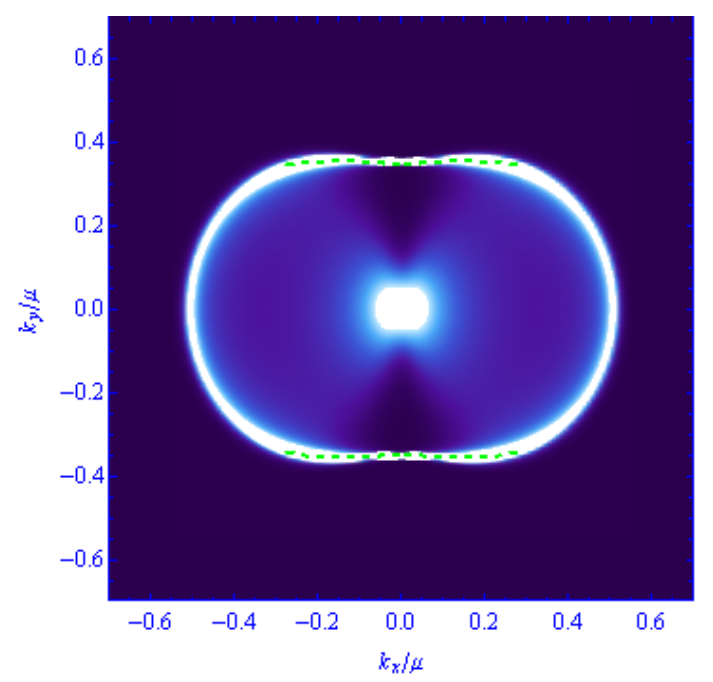

(e) $T=0.28 T_{c}$

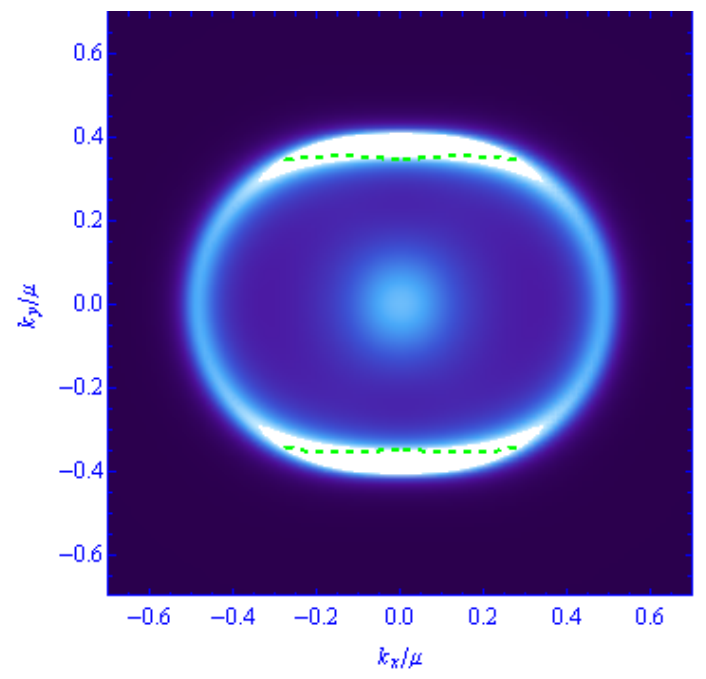

(b) $T=0.79 T_{c}$

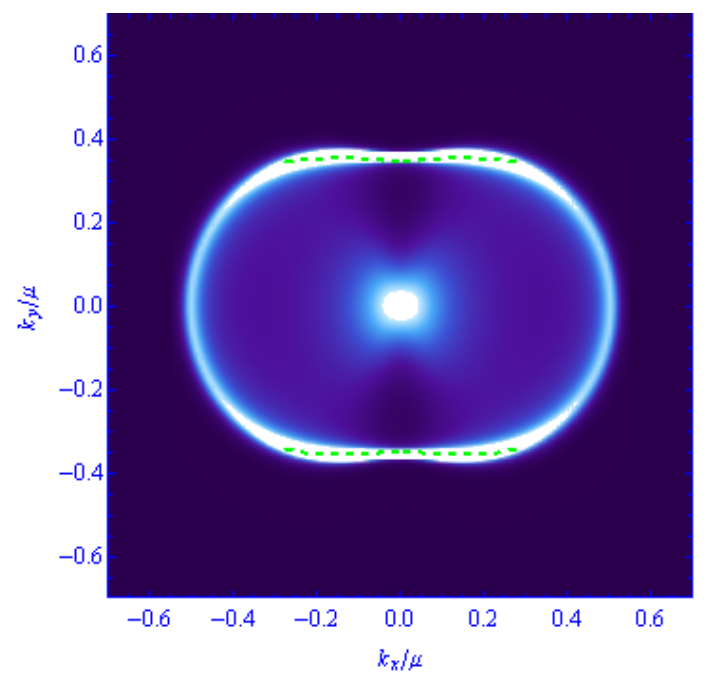

(d) $T=0.38 T_{c}$

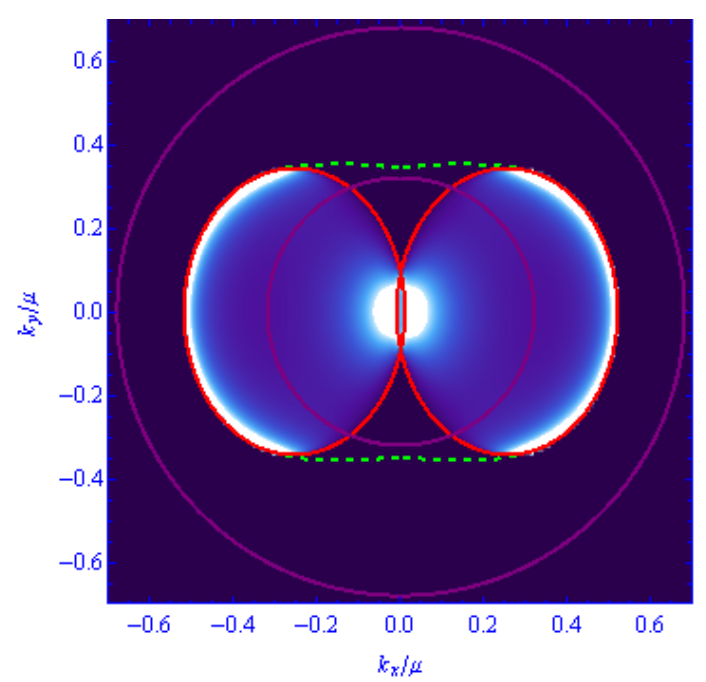

(f) $T=0$

Figura 48: La función espectral para diferentes temperaturas y para un valor fijo de $\lambda=0.62=\lambda_{c}$, $\omega=0.18 \mu$. Se observa la presencia de simetría esférica a $T=T_{c}$. Las líneas punteadas indican el lugar de los modos normales. Los círculos violetas y rojos representa los conos de Dirac UV y IR respectivamente. 


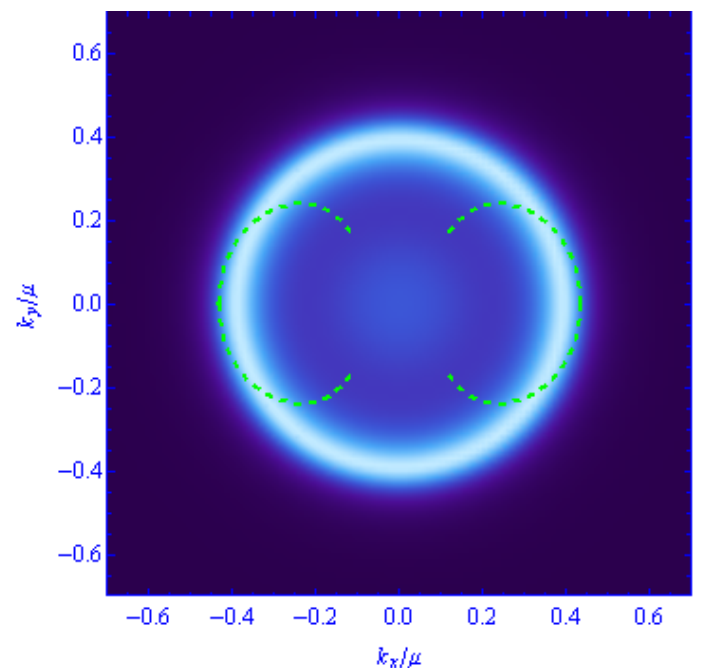

(a) $T=T_{c}$

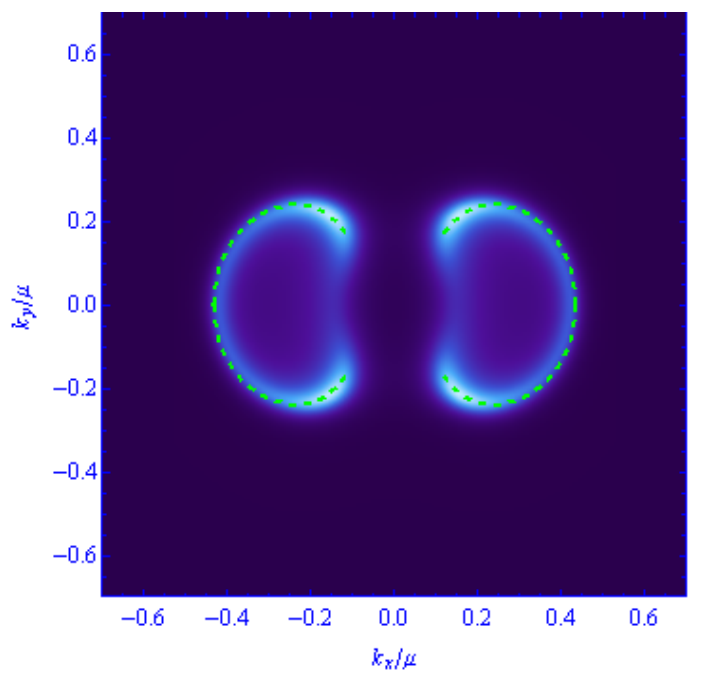

(c) $T=0.60 T_{c}$

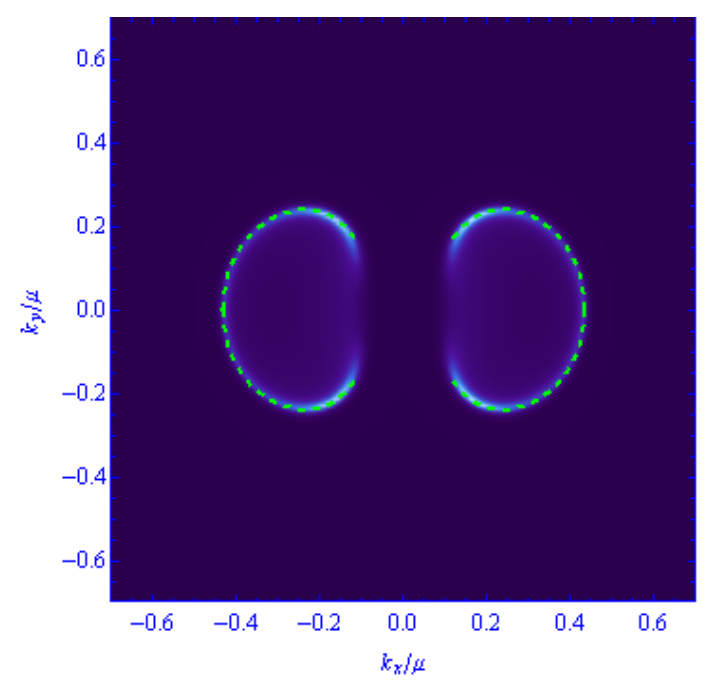

(e) $T=0.28 T_{c}$

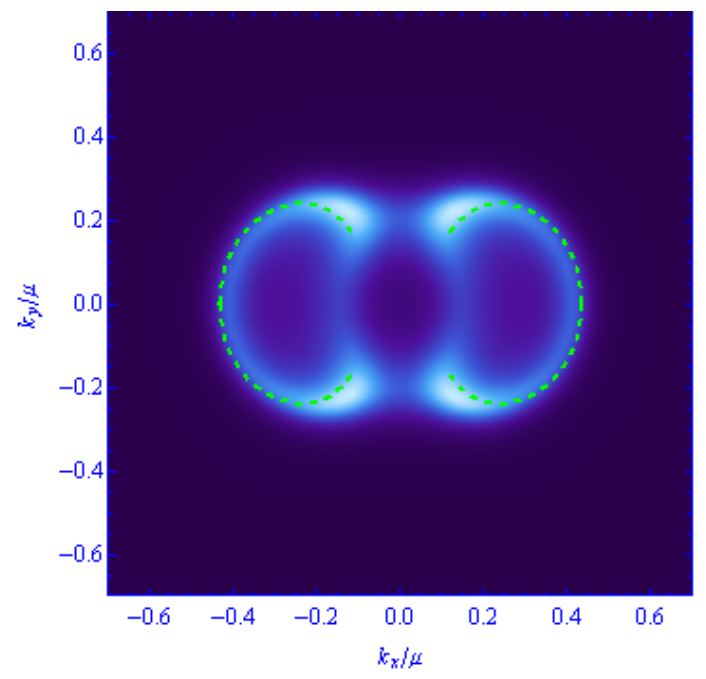

(b) $T=0.79 T_{c}$

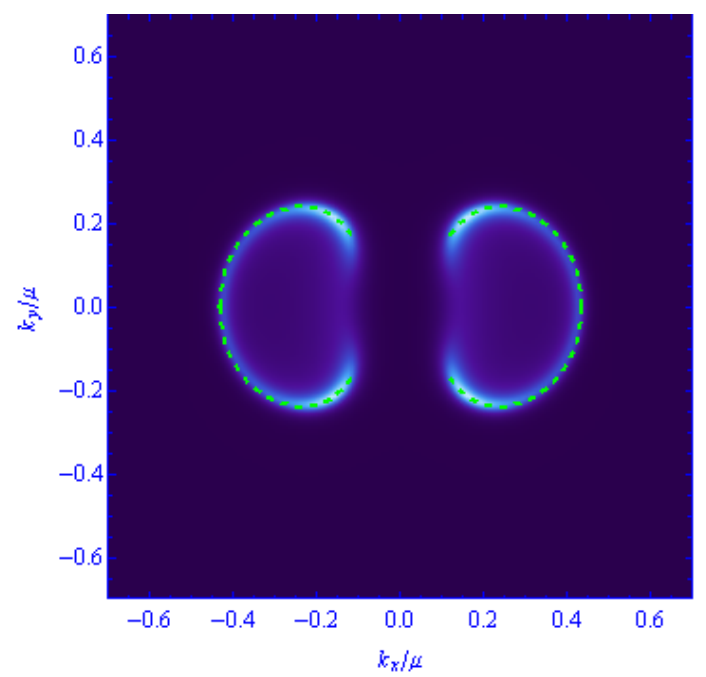

(d) $T=0.38 T_{c}$

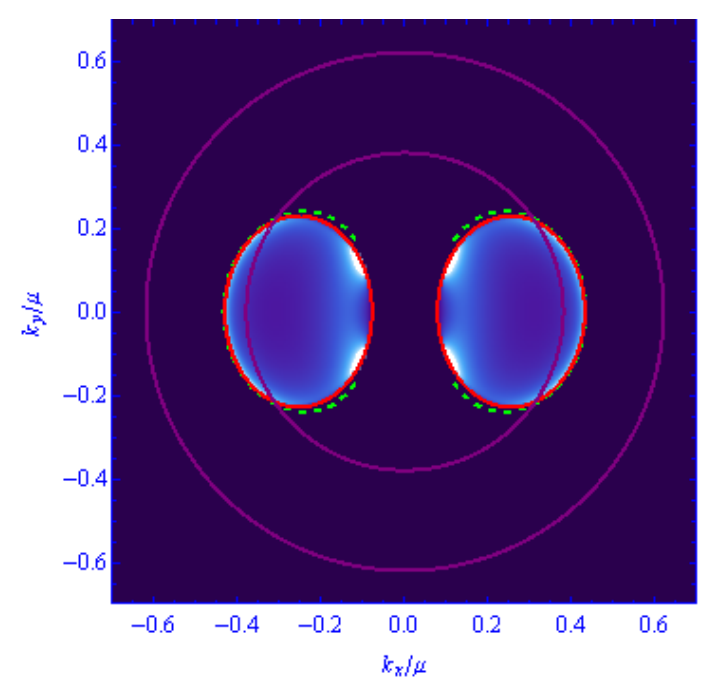

(f) $T=0$

Figura 49: La función espectral para diferentes temperaturas y para un valor fijo de $\lambda=0.62=\lambda_{c}$, $\omega=0.12 \mu$. Se observa la presencia de simetría esférica a $T=T_{c}$. Las líneas punteadas indican el lugar de los modos normales. Los círculos violetas y rojos representa los conos de Dirac UV y IR respectivamente. 


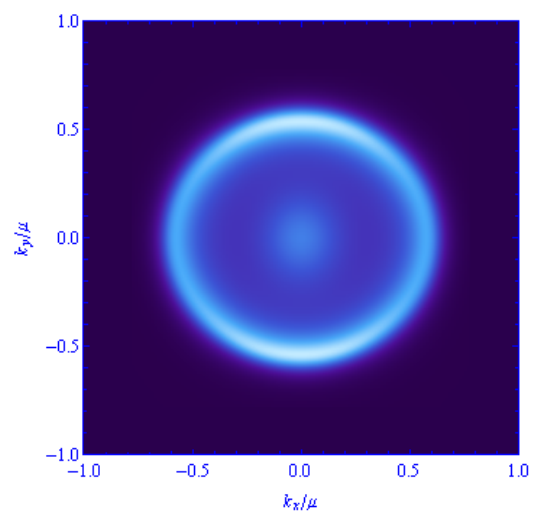

(a) $T=0.80 T_{c}$

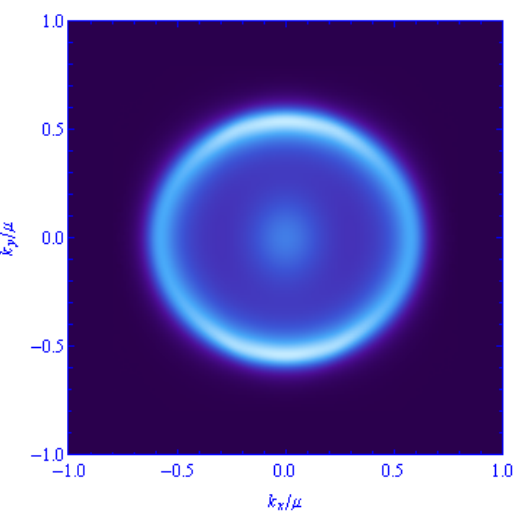

(b) $T=0.40 T_{c}$

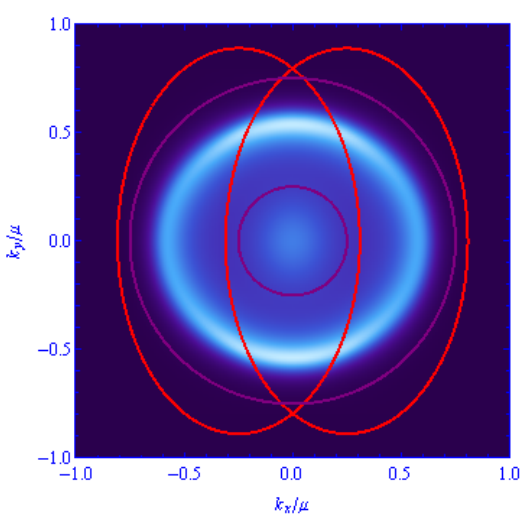

(c) $T=0$

Figura 50: La función espectral para diferentes temperaturas y para un valor fijo de $\lambda=0.80>\lambda_{c}$, $\omega=0.25 \mu$. Permanece prácticamente sin cambiar para el rango entero de temperaturas. Los círculos violetas y rojos representa los conos de Dirac UV y IR respectivamente.

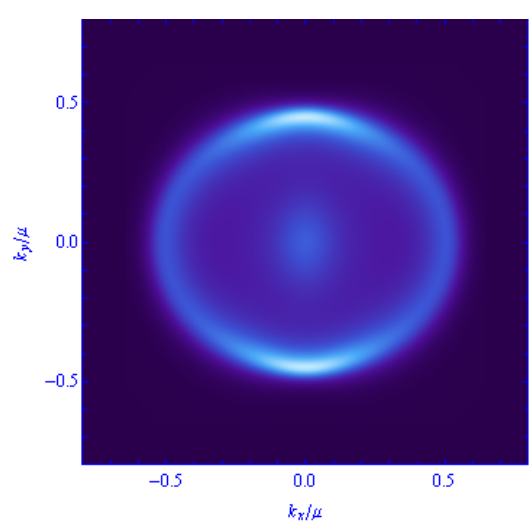

(a) $T=0.80 T_{c}$

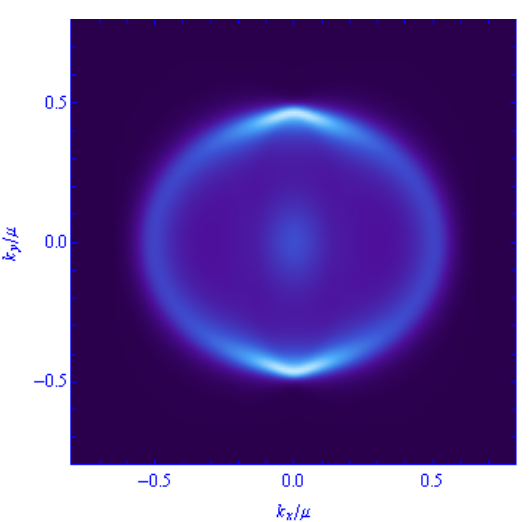

(b) $T=0.40 T_{c}$

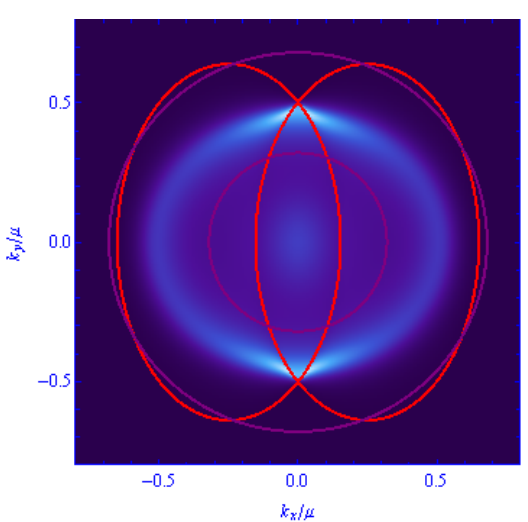

(c) $T=0$

Figura 51: La función espectral para diferentes temperaturas y para un valor fijo de $\lambda=0.80>\lambda_{c}$, $\omega=0.18 \mu$. Permanece prácticamente sin cambiar para el rango entero de temperaturas. Los círculos violetas y rojos representa los conos de Dirac UV y IR respectivamente.

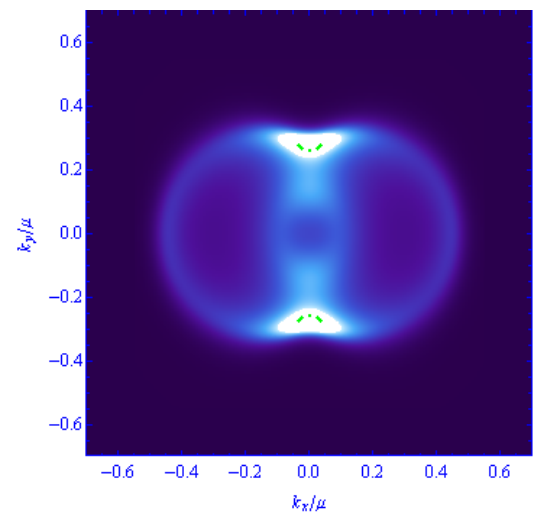

(a) $T=0.80 T_{c}$

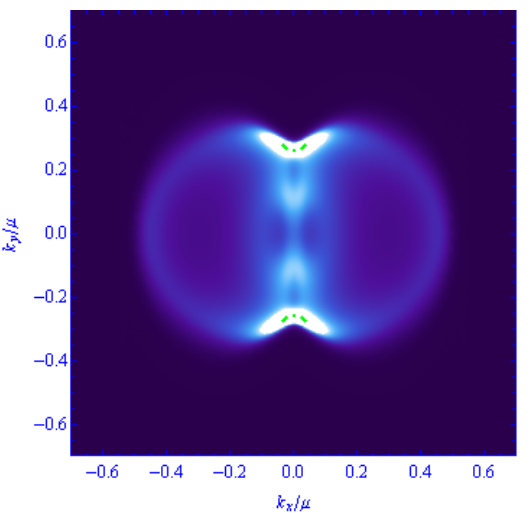

(b) $T=0.40 T_{c}$

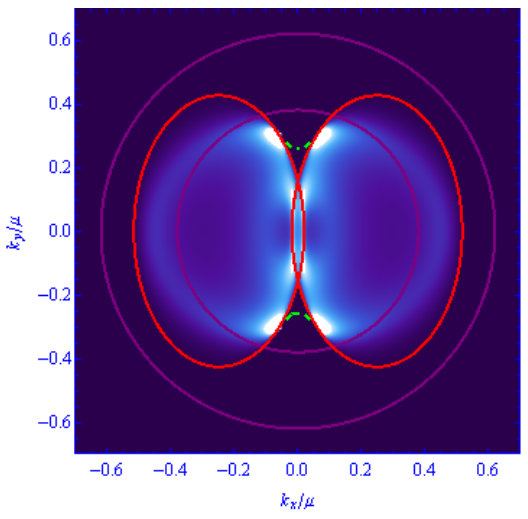

(c) $T=0$

Figura 52: La función espectral para diferentes temperaturas y para un valor fijo de $\lambda=0.80>\lambda_{c}$, $\omega=0.12 \mu$. Permanece prácticamente sin cambiar para el rango entero de temperaturas. Los círculos violetas y rojos representa los conos de Dirac UV y IR respectivamente. 


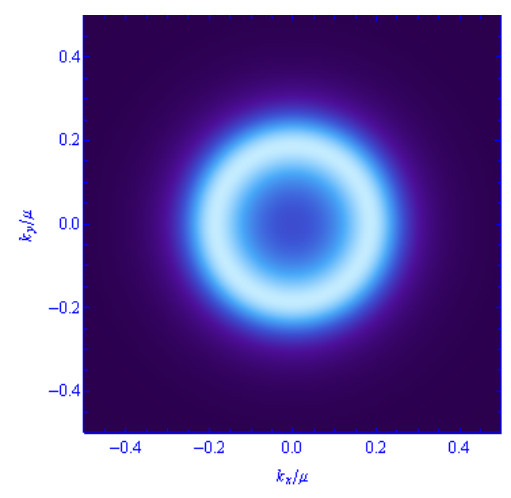

(a) $\omega=0, T=T_{c}$

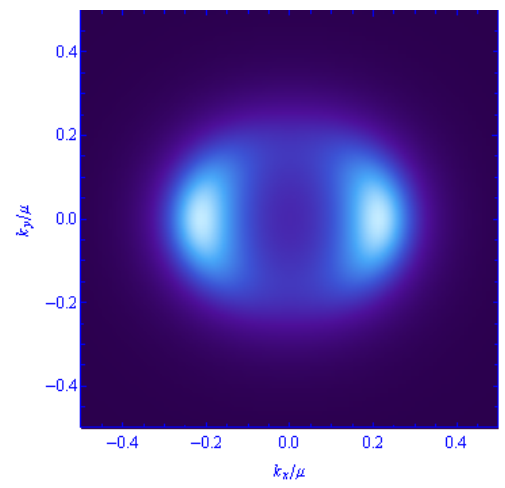

(b) $\omega=0, T=0.95 T_{c}$

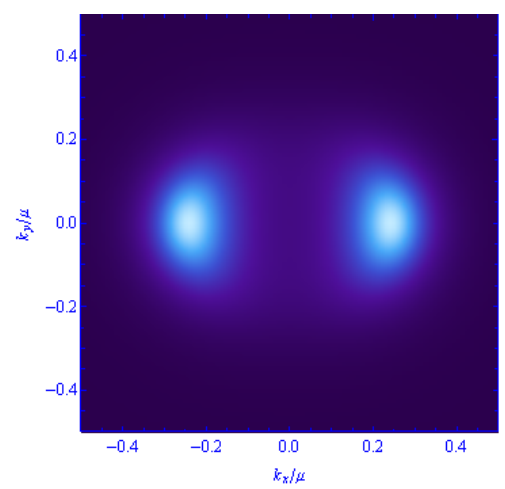

(c) $\omega=0, T=0.85 T_{c}$

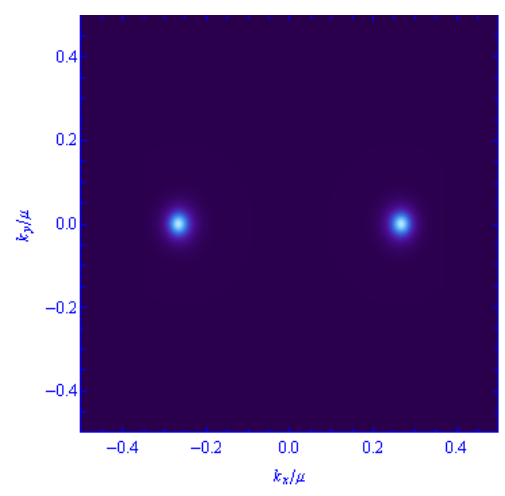

(d) $\omega=0, T=0.20 T_{c}$

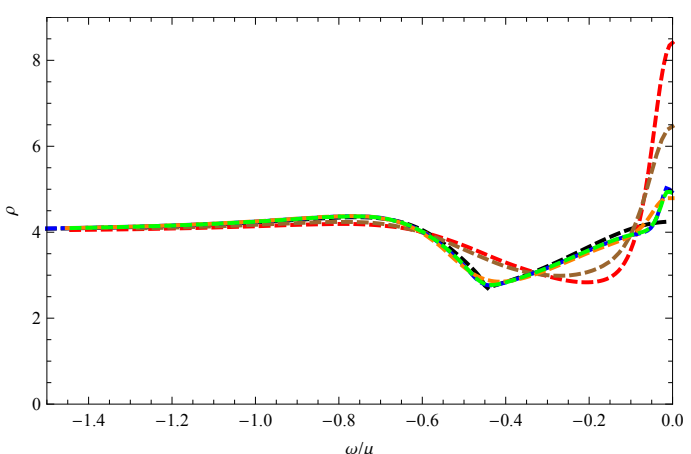

(1)

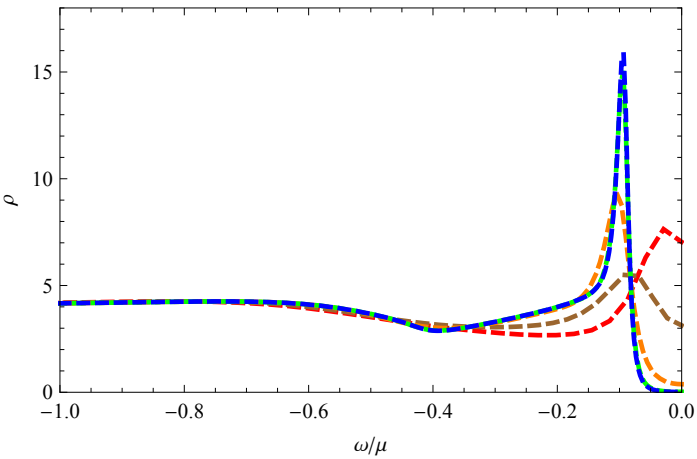

(2)

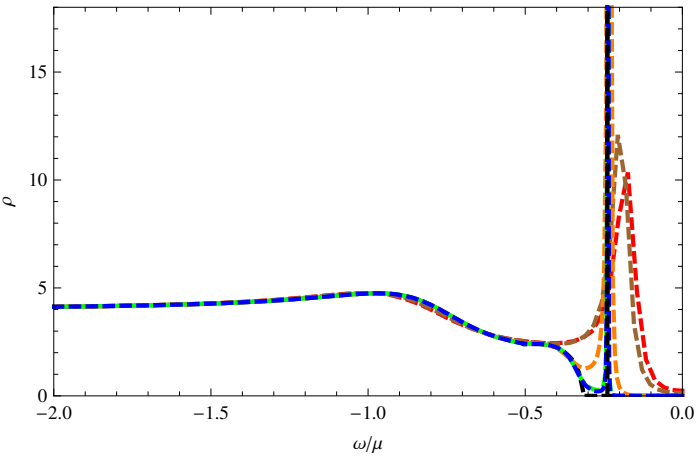

(3)

Figura 53: jajajaLa función espectral para un valor fijo de $\lambda=0.40<\lambda_{c}$. Izquierda : La superficie espectral $\omega=0$ a diferentes temperaturas. Derecha: (1) $\rho$ como función de $\omega$ a $\left(k_{x}, k_{y}\right)=\left(k_{*}, 0\right)$, notemos que la curva negra $(T=0)$ también presenta un pico tipo delta a $\omega=0$ el cual no es representado en la figura ; (2) $\rho$ como función de $\omega$ a $\left(k_{x}, k_{y}\right)=(0.20,0.10) \mu$; (3) $\rho$ como función de $\omega$ a $\left(k_{x}, k_{y}\right)=(0.20,0.40) \mu$. Cada gráfico en esta figura corresponden a las temperaturas: $T=T_{c}$ (roja), $T=0.97 T_{c}$ (marrón), $T=0.86 T_{c}$ (naranja), $T=0.43 T_{c}$ (verde), $T=0.21 T_{c}$ (azul), $T=0$ (negra). 


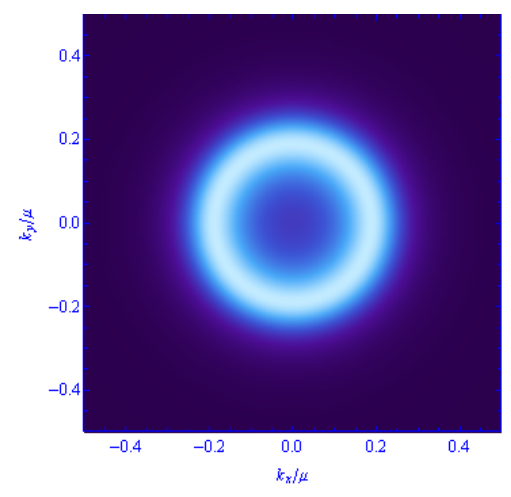

(a) $\omega=0, T=T_{c}$

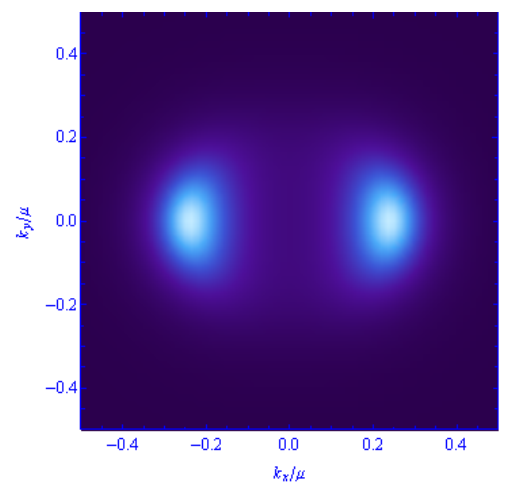

(b) $\omega=0, T=0.97 T_{c}$

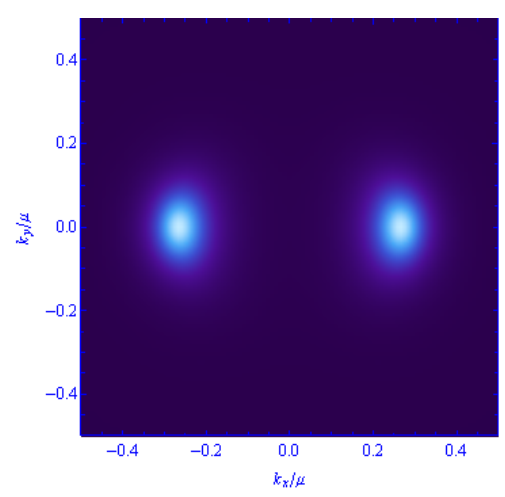

(c) $\omega=0, T=0.79 T_{c}$

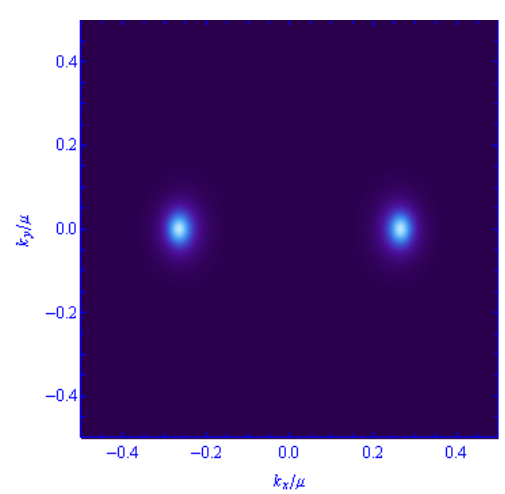

(d) $\omega=0, T=0.41 T_{c}$

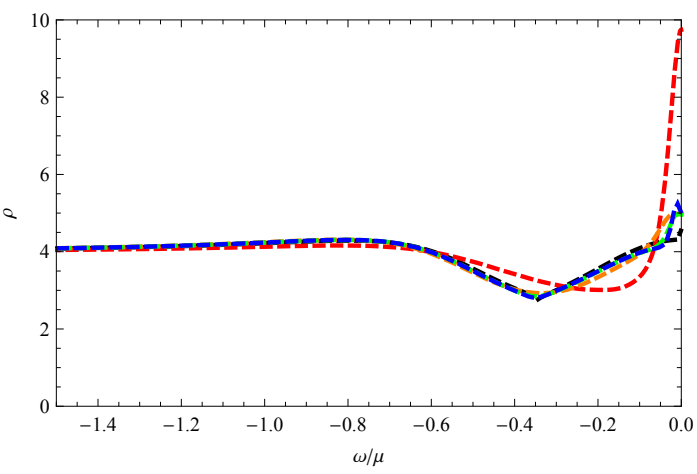

(1)

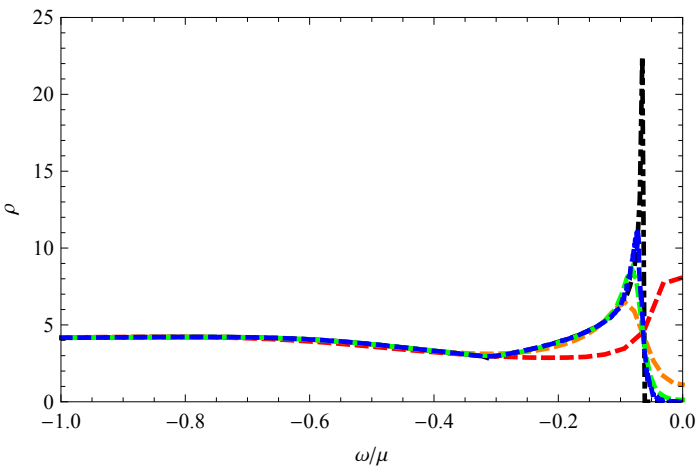

(2)

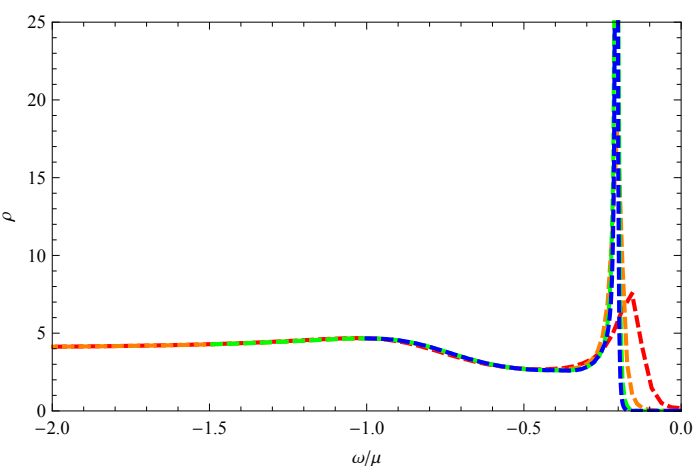

(3)

Figura 54: La función espectral para un valor fijo de $\lambda=\lambda_{c}=0.62$. Izquierda: La superficie espectral $\omega=0$ a diferentes temperaturas. Derecha: (1) $\rho$ como función de $\omega$ a $\left(k_{x}, k_{y}\right)=\left(k_{*}, 0\right)$, notemos que la curva negra $(T=0)$ también presenta un pico tipo delta a $\omega=0$ el cual no es representado en la figura ; $(2) \rho$ como función de $\omega$ a $\left(k_{x}, k_{y}\right)=(0.20,0.10) \mu ;(3) \rho$ como función de $\omega$ a $\left(k_{x}, k_{y}\right)=(0.20,0.40) \mu$. Cada gráfico en esta figura corresponden a las temperaturas: $T=T_{c}$ (roja), $T=0.97 T_{c}$ (violeta), $T=0.76 T_{c}$ (naranja), $T=0.38 T_{c}$ (verde), $T=0.28 T_{c}$ (azul), $T=0$ (negra). 


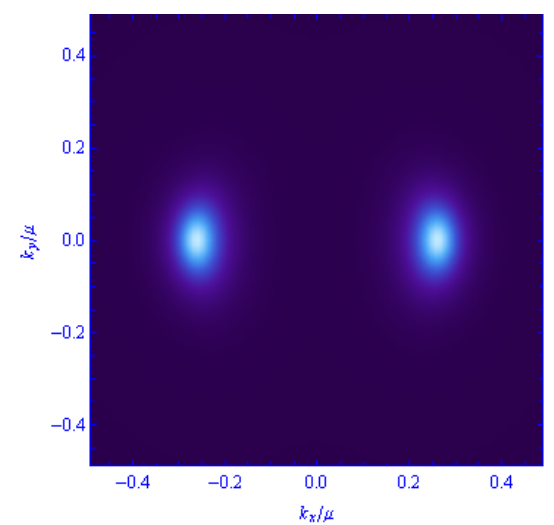

(a) $\omega=0, T=T_{c}$

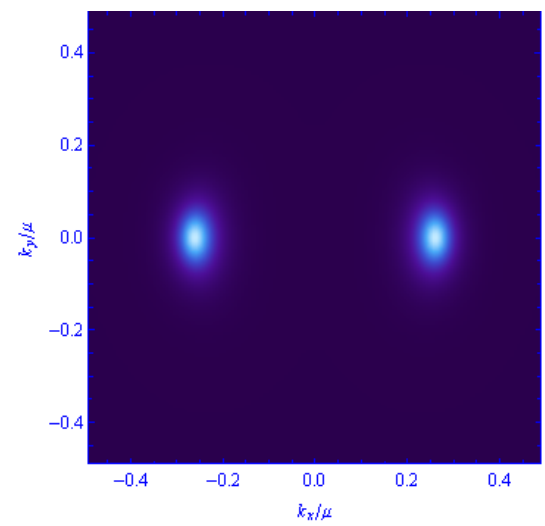

(b) $\omega=0, T=0.80 T_{c}$

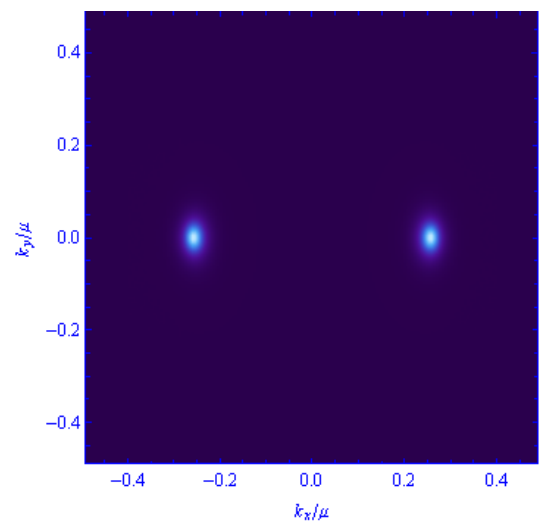

(c) $\omega=0, T=0.40 T_{c}$

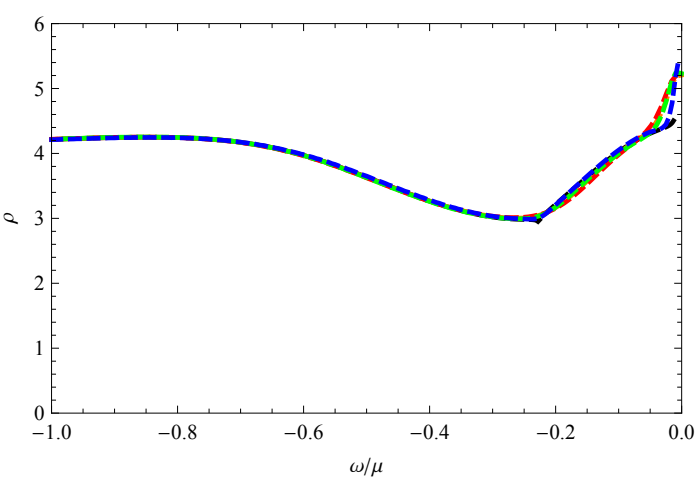

(1)

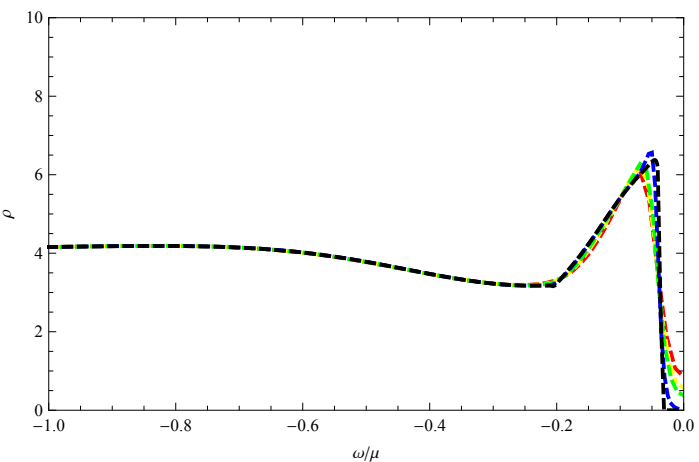

$(2)$

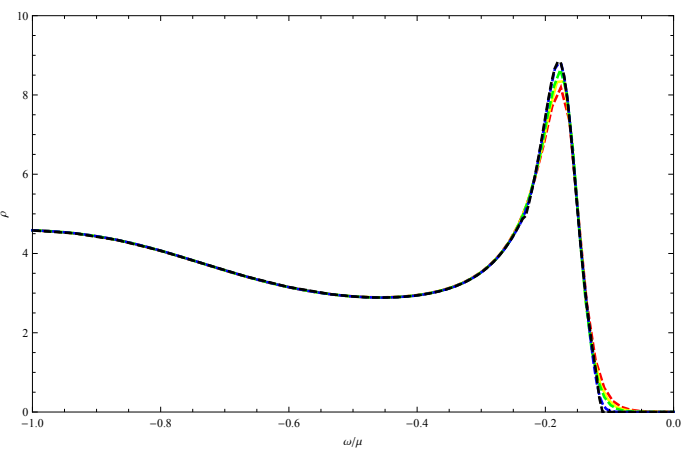

(3)

Figura 55: La función espectral para un valor fijo de $\lambda=0.80>\lambda_{c}$. Izquierda: La superficie espectral $\omega=0$ a diferentes temperaturas. Derecha: (1) $\rho$ como función de $\omega$ a $\left(k_{x}, k_{y}\right)=\left(k_{*}, 0\right)$, notemos que la curva negra $(T=0)$ también presenta un pico tipo delta a $\omega=0$ el cual no es representado en la figura ; $(2) \rho$ como función de $\omega$ a $\left(k_{x}, k_{y}\right)=(0.20,0.10) \mu ;(3) \rho$ como función de $\omega$ a $\left(k_{x}, k_{y}\right)=(0.20,0.40) \mu$. Cada gráfico en esta figura corresponden a las temperaturas: $T=T_{c}$ (roja), $T=0.90 T_{c}$ (naranja), $T=0.86 T_{c}$ (amarillo), $T=0.80 T_{c}$ (verde), $T=0.4 T_{c}$ (azul), $T=0$ (negra). 


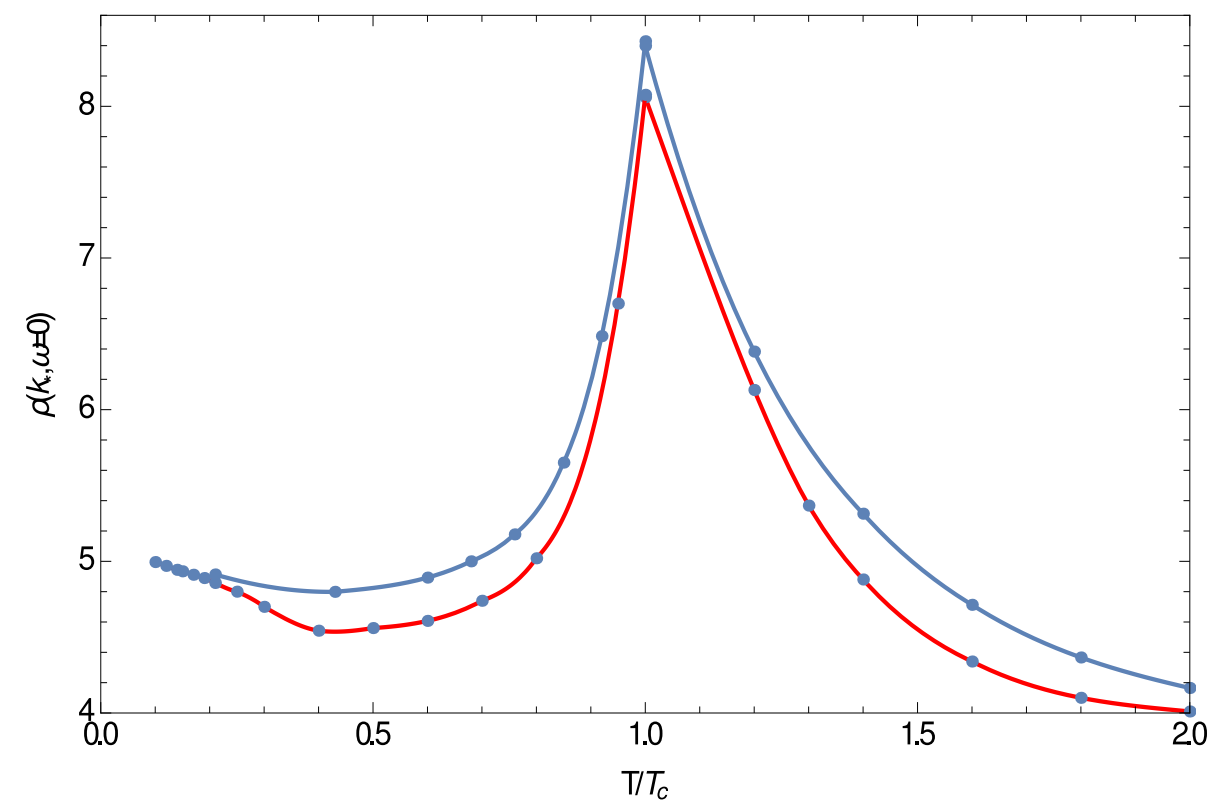

Figura 56: El valor de $\rho\left(k_{*}, \omega=0\right)$ es mostrado como función de $T / T_{c}$. La curva azul corresponde a $\lambda=1 /(8 \sqrt{2})$, mientras que la curva roja se corresponde a $\lambda=0.4$. 


\subsection{Discusión}

En este trabajo hemos estudiado la función espectral de los grados de libertad fermiónicos sobre un superconductor de onda- $p$ holográfico teniendo en cuenta el acoplamiento gravitacional, a temperatura y entropía finita extendiendo trabajos previos en la literatura.

A temperatura nula, los modos normales existen dentro de los conos de Dirac UV y fuera de los conos IR. Este comportamiento es consistente con la aparición de arcos donde la intersección de los conos de Dirac UV y IR no permiten una superficie de Fermi cerrada.

Los modos normales pueden ser interpretados como un modelo donde una relación entre la frecuencia y los momentos pueden ser entendidos como una relación de dispersión de cuasi-partículas.

El soporte de la densidad espectral esta completamente contenida dentro de los conos de Dirac IR y las fuertes divergencias en los modos normales.

A temperatura finita, la región donde la función espectral es numéricamente apreciable se reduce cuando la temperatura disminuye, los picos en los modos normales se vuelven mucho mas agudos. Hay un rompimiento de la invarianza rotacional que se vuelve mas fuerte a medida que la temperatura disminuye.

No obstante nuestro resultados muestran que tal dependencia con la temperatura es suave conforme el acoplamiento gravitacional es incrementado.

La densidad espectral evaluada en el momento de Fermi presenta un pico de cuasi-partícula para una frecuencia nula, esto puede ser interpretado como la vida media de la cuasi-partícula. En los gráficos, vemos que la cuasi-partícula se estabiliza cuando la temperatura disminuye, que es lo que se espera debido a la presencia de los modos normales y también cuando la temperatura se aproxima a la temperatura crítica de la transición de fase superconductora

\subsection{Apéndice B1: Acerca de la positividad de la función espectral}

En este apéndice estableceremos las propiedad de no negatividad de la función espectral siguiendo el análisis del caso $T=0$ en [14] y extendiendo los resultados para el caso $T>0$. El punto de partida es la conservación de la corriente (on-shell)

$$
J^{A}(x, z) \equiv i \bar{\Psi}(x ; z) \Gamma^{A} \Psi(x ; z) \quad \longrightarrow \quad J^{A}(z ; k)=i \frac{z^{3}}{s \sqrt{f}} \bar{\psi}_{k}(z) \Gamma^{A} \psi_{k}(z)
$$

donde sobre el lado derecho usamos el ansatz introducido en (306). La ecuación de conservación se escribe,

$$
\begin{aligned}
D_{A} J^{A}(z ; k) & =\frac{z^{4}}{s} Q^{\prime}(z ; k)=0 \\
Q(z ; k) & \equiv-2 \operatorname{Im}\left(\bar{\psi}_{k}^{+}(z) \psi_{k}^{-}(z)\right)=-2 \operatorname{Im}\left(\vec{u}_{k}(z)^{\dagger} \mathbf{C} \vec{v}_{k}(z)\right)=-2 \operatorname{Im}\left(\vec{u}_{k}(z)^{\dagger} \mathbf{S}(z)^{-1} \vec{u}_{k}^{\prime}(z)\right)(34
\end{aligned}
$$

Aquí la matriz $\mathbf{S}(z) \equiv-i \mathbf{U}(z) \mathbf{C}$ es hermítica, $\mathbf{S}(z)^{\dagger}=\mathbf{S}(z)$, y en la segunda igualdad hemos introducido la notación (310) con la matriz $\mathbf{C}$ dada en (305), mientras que para la ultima igualdad usamos las ecuaciones de movimiento (312).

De (346) concluimos que, cuando evaluamos on-shell, $Q(z ; k)=Q(k)$ es una cantidad conservada, incluso si no sabemos la solución explicita a las ecuaciones de movimiento podemos evaluarla, en los límites conocidos.

En primer lugar consideremos el límite UV; con ayuda del comportamiento asintótico (331), la relación (340) y la definición (343) obtenemos

$$
Q_{U V}(k) \equiv Q(0 ; k)=i\left(\mathbf{C} \vec{u}_{k}^{(U V)}\right)^{\dagger}\left(\mathbf{G}_{R}(k)-\mathbf{G}_{R}(k)^{\dagger}\right) \mathbf{C} \vec{u}_{k}^{(U V)}
$$

Si asumimos que $Q_{U V}(k) \geq 0$, y siendo el vector $\vec{u}_{k}^{(U V)}$ arbitrario, debemos concluir que la matriz hermítica $i\left(\mathbf{G}_{R}(k)-\mathbf{G}_{R}(k)^{\dagger}\right)$ es no negativa. De la definición (344) concluimos que,

$$
\rho\left(\omega ; k_{x}, k_{y}\right) \equiv \operatorname{Tr} \frac{i}{2}\left(\mathbf{G}_{R}(k)-\mathbf{G}_{R}(k)^{\dagger}\right) \geq 0
$$

siendo estrictamente cero si todos los autovalores son cero y entonces $Q_{U V}=0$

En segundo lugar consideremos el límite UR: este caso se divide en dos. Cuando la entropía desaparece el comportamiento asintótico para $z \rightarrow \infty$ es de la forma

$$
\vec{u}_{k}(z) \simeq e^{-\kappa_{+} z}\left(\begin{array}{c}
b_{1} \\
b_{2} \\
-b_{1} \\
-b_{2}
\end{array}\right)+e^{-\kappa_{-} z}\left(\begin{array}{l}
b_{3} \\
b_{4} \\
b_{3} \\
b_{4}
\end{array}\right)
$$


donde el vector $\vec{b}^{t}=\left(b_{1}, b_{2}, b_{3}, b_{4}\right)$ es leído de $(320)$ en términos de $\vec{u}_{k}^{(i r)}$ pero tal relación no es relevante aquí. De (346) obtenemos

$$
\begin{aligned}
Q_{I R}(k) & =\left.Q(z ; k)\right|_{z \rightarrow \infty} \\
& =\lim _{z \rightarrow \infty}\left(2 \operatorname{Im}\left(\kappa_{+}\right)\left|e^{-\kappa_{+} z}\right|^{2}\left(\begin{array}{c}
b_{1} \\
b_{2} \\
-b_{1} \\
-b_{2}
\end{array}\right) \mathbf{S}_{I R}^{-1}\left(\begin{array}{c}
b_{1} \\
b_{2} \\
-b_{1} \\
-b_{2}
\end{array}\right)+2 \operatorname{Im}\left(\kappa_{-}\right)\left|e^{-\kappa_{-} z}\right|^{2}\left(\begin{array}{c}
b_{3} \\
b_{4} \\
b_{3} \\
b_{4}
\end{array}\right)^{\dagger} \mathbf{S}_{I R}^{-1}\left(\begin{array}{c}
b_{3} \\
b_{4} \\
b_{3} \\
b_{4}
\end{array}\right)\right. \\
& \left.+2 \operatorname{Im}\left(\kappa_{+} e^{-\left(\kappa_{+}+\kappa_{-}{ }^{*}\right) z}\left(\begin{array}{c}
b_{3} \\
b_{4} \\
b_{3} \\
b_{4}
\end{array}\right) \mathbf{S}_{I R}^{-1}\left(\begin{array}{c}
b_{1} \\
b_{2} \\
-b_{1} \\
-b_{2}
\end{array}\right)+\kappa_{-} e^{-\left(\kappa_{+}{ }^{*}+\kappa_{-}\right) z}\left(\begin{array}{c}
b_{1} \\
b_{2} \\
-b_{1} \\
-b_{2}
\end{array}\right) \mathbf{S}_{I R}^{-1}\left(\begin{array}{c}
b_{3} \\
b_{4} \\
b_{3} \\
b_{4}
\end{array}\right)\right)\right)
\end{aligned}
$$

donde

$$
\left.\mathbf{S}_{I R} \equiv \mathbf{S}\right|_{z \rightarrow \infty}=\left(\begin{array}{cc}
\mathbf{S}_{\mathbf{1}} & \mathbf{S}_{\mathbf{2}} \\
\mathbf{S}_{\mathbf{2}} & \mathbf{S}_{\mathbf{1}}
\end{array}\right) \quad ; \quad \mathbf{S}_{\mathbf{1}} \equiv-\frac{\omega}{s_{0}} \mathbf{1}-\frac{k_{y}}{g_{0}} \sigma_{\mathbf{1}}+g_{0} k_{x} \sigma_{\mathbf{3}} \quad, \quad \mathbf{S}_{\mathbf{2}} \equiv-\frac{g_{0} w_{0}}{2} \sigma_{\mathbf{3}}
$$

Por cómputos directos de (350), y usando el hecho que los autovalores de las matrices $\mathbf{S}_{ \pm} \equiv \mathbf{S}_{\mathbf{1}} \mp \mathbf{S}_{\mathbf{2}}$ son reales y son dados respectivamente por,

$$
-\frac{\omega}{s_{0}} \pm \sqrt{\left(\frac{\omega}{s_{0}}\right)^{2}+\kappa_{+}{ }^{2}} \quad, \quad-\frac{\omega}{s_{0}} \pm \sqrt{\left(\frac{\omega}{s_{0}}\right)^{2}+\kappa_{-}{ }^{2}}
$$

con $\kappa_{ \pm}^{2}$ definidos en (316), es un poco tedioso pero directo mostrar que $Q_{I R}(k)>0$ excepto cuando estamos fuera de ambos conos de Dirac IR, i.e. $\kappa_{ \pm}{ }^{2}>0$, caso en el cual $Q_{I R}(k)=0$. Entonces ya que la conservación de la corriente implica $Q_{U V}(k)=Q_{I R}(k)=Q(k)$ podemos concluir que efectivamente $\rho(k) \geq 0$, siendo cero afuera de ambos conos de Dirac IR o en otras palabras $\rho$ es estrictamente positiva dentro de algún cono de Dirac IR y nulo fuera de ambos de ellos.

Cuando $T>0$ la cosa se vuelve mucho mas simple; el comportamiento para $z \rightarrow 1^{-}$es de la forma,

$$
\vec{u}_{k}(z) \simeq \exp ^{-i \frac{\omega}{4 \pi T}} \ln (1-z) \vec{b}
$$

donde $\vec{b}$ es leído de (328) en términos de $\vec{u}_{k}^{(i r)}$. De (346) obtenemos,

$$
Q_{I R}(k)=2 \vec{b}^{\dagger} \vec{b}>0
$$

Entonces encontramos que a temperatura finita la función espectral debe ser estrictamente positiva en cualquier lugar en el espacio de momentos.

\subsection{Apéndice B2: Sobre la función espectral en un espacio-tiempo conforme}

Este apéndice intenta clarificar la relación entre las condiciones de borde entrantes, la preincripción en $\omega$ y las positividad de la función espectral cuando tenemos un espacio-tiempo conforme arbitrario.

Consideraremos la siguiente métrica, solución a las ecuaciones de movimiento (292)

$$
\begin{aligned}
G & =\frac{1}{z^{2}}\left(-d t^{2}+d \vec{x}^{2}+d z^{2}\right) \\
\mathbf{A} & =\left(d t \phi+d x^{1} W_{1}+d x^{2} W_{2}\right) \tau_{0} .
\end{aligned}
$$

donde $\left(\phi, W_{1}, W_{2}\right)$ constantes y tomaremos la subalgebra de Cartan en la dirección $\tau_{0}$.

Trabajando en el espacio de momentos, $\Psi(x, z)=z^{3 / 2} e^{i k_{\mu} x^{\mu}}\left(\begin{array}{c}\psi_{k}^{+}(z) \\ \psi_{k}^{-}(z)\end{array}\right)$ y después de introducir la notación (310), las ecuaciones de movimiento resultan como en (312) con la matriz constante $\mathbf{U}$ definida por

$$
\mathbf{U}=\left(\begin{array}{cc}
\mathbf{U}_{+} & \mathbf{0} \\
\mathbf{0} & \mathbf{U}_{-}
\end{array}\right) \quad ; \quad \mathbf{U}_{ \pm} \equiv-\lambda_{a}^{ \pm} \gamma^{a}
$$

donde

$$
\lambda_{0}^{ \pm}=-\omega \mp \frac{\phi}{2} \quad, \quad \lambda_{1}^{ \pm}=k_{x} \mp \frac{W_{1}}{2} \quad, \quad \lambda_{2}^{ \pm}=k_{y} \mp \frac{W_{2}}{2}
$$




$$
\lambda_{ \pm}^{2} \equiv \eta^{a b} \lambda_{a}^{ \pm} \lambda_{b}^{ \pm}=-\left(\omega \pm \frac{\phi}{2}\right)^{2}+\left(k_{x} \mp \frac{W_{x}}{2}\right)^{2}+\left(k_{y} \mp \frac{W_{y}}{2}\right)^{2}
$$

La solución general de (312) es

$$
\vec{u}_{k}(z)=\left(\begin{array}{c}
e^{\sqrt{\lambda_{+}^{2}} z} \mathbf{a}^{+}+e^{-\sqrt{\lambda_{+}^{2}} z} \mathbf{b}^{+} \\
e^{\sqrt{\lambda_{-} 2} z} \mathbf{a}^{-}+e^{-\sqrt{\lambda_{-}^{2}} z} \mathbf{b}^{-}
\end{array}\right) \quad ; \quad \vec{v}_{k}(z)=i\left(\begin{array}{cc}
\frac{\mathbf{U}_{+}}{\lambda_{+}^{2}} & \mathbf{0} \\
\mathbf{0} & \frac{\mathbf{U}_{-}}{\lambda_{-}^{2}}
\end{array}\right) \vec{u}_{k}^{\prime}(z)
$$

Vamos a definir,

$$
\sqrt{\lambda_{ \pm}^{2}}= \begin{cases}\lambda_{ \pm} \geq 0 & , \quad \lambda_{ \pm}^{2} \geq 0 \\ i \operatorname{sign}\left(\lambda_{0}^{ \pm}\right) \sqrt{-\lambda_{ \pm}^{2}} & , \quad \lambda_{ \pm}^{2}<0\end{cases}
$$

y tomamos las condiciones de borde $\mathbf{a}^{ \pm}=\mathbf{0}$; es claro que en el primer caso es solo una condición suave cuando $z \rightarrow \infty$. Con esta condición de borde la solución puede ser escrita

$$
\begin{aligned}
\vec{u}_{k}(z) & =\left(\begin{array}{cc}
e^{-\sqrt{\lambda_{+}^{2}} z} \mathbf{1} & \mathbf{0} \\
\mathbf{0} & e^{-\sqrt{\lambda_{-}^{2}} z} \mathbf{1}
\end{array}\right) \vec{u}_{k}^{(0)} \\
\vec{v}_{k}(z) & =-i\left(\begin{array}{cc}
e^{-\sqrt{\lambda_{+}} z} z & \mathbf{0} \\
\mathbf{0} & e^{-\sqrt{\lambda_{-}^{2}} z} \mathbf{1}
\end{array}\right)\left(\begin{array}{cc}
\frac{\mathbf{U}_{+}}{\sqrt{\lambda_{+}^{2}}} & \mathbf{0} \\
\mathbf{0} & \frac{\mathbf{U}_{-}}{\sqrt{\lambda_{-}}}
\end{array}\right) \vec{u}_{k}^{(0)}
\end{aligned}
$$

De la definición (340) y usando la receta de AdS/CFT (343) leemos la función de Green retardada

$$
\mathbf{G}_{\mathbf{R}}(k)=\left(\begin{array}{cc}
-\frac{\lambda_{a}^{+}}{\sqrt{\lambda_{+}^{2}}} \gamma^{a} \gamma^{0} & \mathbf{0} \\
\mathbf{0} & -\frac{\lambda_{a}^{-}}{\sqrt{\lambda_{-}^{2}}} \gamma^{a} \gamma^{0}
\end{array}\right)
$$

La funcion espectral es entonces obtenida de (344),

$$
\rho\left(\omega, k_{x}, k_{y}\right)=2\left(\frac{\left|\omega+\frac{\phi}{2}\right|}{\sqrt{-\lambda_{+}{ }^{2}}} \theta\left(-\lambda_{+}{ }^{2}\right)+\frac{\left|\omega-\frac{\phi}{2}\right|}{\sqrt{{-\lambda_{-}}^{2}}} \theta\left(-\lambda_{-}{ }^{2}\right)\right)
$$

Vemos que la b.c. adoptada para $z \rightarrow \infty$ junto con la introducción en la ecuación (359) de el signo correcto en la definición de la raiz de $\lambda_{ \pm}{ }^{2}$ en la región donde ellas son negativas, i.e. dentro de los conoes de Dirac (y donde la función espectral es distinta de cero) es lo que asegura la positividad de la función espectral y cuando $\phi=0$, el caso relevante en el límite IR a entropía nula considerado en el artículo, ellas coinciden con las ingoing b.c en la región $\kappa_{ \pm}^{2}<0$ considerada en la subsección 6.6.1.

Como ultima nota, es fácil ver que estas b.c efectivamente siguen de la preinscripción de suavidad después de cambiar $\omega \rightarrow \omega+i \epsilon$ con $\epsilon \rightarrow 0^{+}$; en efecto si asumimos que $\lambda_{ \pm}{ }^{2}<0$ tenemos,

$$
\left.\sqrt{\lambda_{ \pm}^{2}}\right|_{\omega \rightarrow \omega+i \epsilon} \approx \sqrt{\lambda_{ \pm}^{2}}+\left.i \frac{\lambda_{0}^{ \pm}}{\sqrt{\lambda_{ \pm}^{2}}} \epsilon \quad \Longrightarrow \quad e^{-\sqrt{\lambda_{ \pm}^{2}} z}\right|_{\omega \rightarrow \omega+i \epsilon} \approx e^{-\sqrt{\lambda_{ \pm}^{2}} z} e^{-i \frac{\lambda_{0}^{ \pm}}{\sqrt{\lambda_{ \pm}^{2}}} \epsilon z}
$$

y entonces la exponencial se comporta bien para $z \rightarrow \infty$ si (359) se mantiene [14]. 


\section{Funciones espectrales en metales holográficos dopados y el cruce en la fase de metal extraño}

\section{Resumen}

Investigamos funciones espectrales en la fase normal de un superconductor de onda- $s$ dopado a temperatura nula usando la correspondencia $A d S / C F T$. Encontramos evidencia de un cruce entre un líquido de Fermi-Landau a una fase de metal extraño al variar el dopaje.

\subsection{Introducción}

Un sistema fuertemente acoplado se encuentra fuera del rango de valides de los métodos tradicionales usados en la comunidad de la Materia Condensada, tales como la teoría de bandas electrónicas y teoría de perturbaciones. En efecto la teoría BCS [89] ha sido exitosa para explicar los superconductores convencionales pero no funciona en el caso de los HTSC. Aunque ha habido importantes progresos en los últimos 30 años (ver por ejemplo [90] y referencias) todavía es necesario un completo entendimiento de los HTSC.

En una serie de experimentos muy interesantes, Chatterjee [92] estudió el espectro de energía a momento fijo en $\mathrm{Bi}_{2} \mathrm{Sr}_{2} \mathrm{CaCu}_{2} \mathrm{O}_{8+\delta}$ para varios valores de dopaje y temperaturas. Para una temperatura fija (ver Fig. 1E [92] ) las curvas de distribución de energía (EDCs) son cualitativamente diferentes conforme el dopaje varia. Para grandes dopajes $\delta>0.17$, se puede ver un pico agudo en la energía de Fermi, indicando excitaciones con vida media grande y por lo tanto la presencia de cuasi-partículas bien definidas como en el estado de líquido de Fermi usual observado en metales convencionales. Por otro lado, conforme el dopaje es disminuido, uno puede observar un ancho espectro en la energía, lo cual significa que la vida media de las excitaciones son muy pequeñas y por lo tanto no hay cuasi-partículas bien definidas (una característica bastante general de sistemas de electrones fuertemente interactuantes) y el sistema se encuentra fuera de la fase de líquido de Fermi, en la llamada fase de metal extraño. No obstante incluso para valores de dopaje pequeños se puede apreciar la aparición de un gap espectral en las EDCs, señalando la entrada en la fase de pseudogap. El estudio de la transición entre la fase de líquido de Fermi y la fase de metal extraño como función del dopaje y la temperatura será el foco de este articulo.

En la última década, la correspondencia AdS/CFT (también conocida como dualidad holográfica o dualidad calibre/gravedad) $[93,94,112]$ ha sido usada como una alternativa para estudiar sistemas fuertemente acoplados en materia condensada (ver por ejemplo referencias [95, 96, 97] y referencias al respecto). Aunque en su forma original [93] la correspondencia es entre una teoría de calibre supersimétrica especifica y una teoría de cuerdas, se espera que una relación similar sea cierta más generalmente, y que un sistema fuertemente acoplado (descripto por una teoría cuántica de campos fuertemente interactuantes) en $D$ - dimensiones sea equivalente (dual) a un sistema débilmente acoplado (en presencia de gravedad) en $D+1$-dimensiones, donde el número de grados de libertad de la teoría cuántica de campos $D$ dimensional es la misma que la de una teoría de la gravedad $D+1$ dimensional. Ejemplos de sistemas donde la correspondencia AdS/CFT ha sido usada incluye: hidrodinamica (también conocida como correspondencia fluido/gravedad) [98, 99], donde ha sido mostrado que existe una mínima cota para el cociente entre la viscosidad y la entropía [100] en un fluido relativista, superconductores holográficos $[101,102,103]$, los cuales capturan muchas características de los superconductores tales como la existencia de una temperatura crítica, un condensado cargado y una brecha en la conductividad óptica, la entropía de entrelazamiento holográfica [104, 105, 106, 107], donde fue propuesta (conjeturada) que la entropía de entrelazamiento en una teoría fuertemente acoplada puede ser calculada en la teoría gravitacional dual computando la superficie de área mínima en el espacio-tiempo curvo.

\subsection{Metales no balanceados}

Los superconductores holográficos no balanceados contienen dos campos de calibre $A$ y $B$, los cuales son duales a la densidad de corriente de los electrones y de las impurezas en la teoría del borde respectivamente. En la fase normal, su dinámica puede ser descripta por un termino de Maxwell para cada campo de calibre acoplado al campo gravitacional,

$$
S_{b}=\frac{1}{2 \kappa^{2}} \int d^{4} x\left(R+\frac{6}{L^{2}}-\frac{L^{2}}{g_{A}^{2}} A_{\mu \nu} A^{\mu \nu}-\frac{L^{2}}{g_{B}^{2}} B_{\mu \nu} B^{\mu \nu}\right)
$$

donde $A_{\mu \nu}=\partial_{\mu} A_{\nu}-\partial_{\nu} A_{\mu}, B_{\mu \nu}=\partial_{\mu} B_{\nu}-\partial_{\nu} B_{\mu}$ y $L$ es la escala AdS relaciona la constante cosmología vía $\Lambda=-3 / L^{2}$. Cualquier solución a las ecuaciónes de movimiento de (364) es una solución al modelo extendido introducido en [108], en la región de parámetros en la cual todos los campos excepto la gravedad y los campos de calibre están apagados. En particular, la geometría del espacio-tiempo dual a la configuración 
de la teoría de campo que vive en el borde de este espacio, con potenciales químicos $\mu_{A}$ para electrones y $\mu_{B}$ para impurezas está dada por el agujero negro AdS plano doblemente cargado, con métrica:

$$
\begin{aligned}
g & =-\frac{r^{2} f(r)}{L^{2}} d t^{2}+\frac{L^{2}}{r^{2} f(r)} d r^{2}+\frac{r^{2}}{L^{2}}\left(d x^{2}+d y^{2}\right) \\
f(r) & \equiv 1-\left(1+\frac{Q_{A}{ }^{2}+Q_{B}^{2}}{r_{h}{ }^{4}}\right)\left(\frac{r_{h}}{r}\right)^{3}+\frac{Q_{A}{ }^{2}+Q_{B}{ }^{2}}{r^{4}}
\end{aligned}
$$

y campos de calibres

$$
A=d t \mu_{A}\left(1-\frac{r_{h}}{r}\right) \quad, \quad B=d t \mu_{B}\left(1-\frac{r_{h}}{r}\right)
$$

El horizonte es fijado a $r=r_{h}$ tal que $f\left(r_{h}\right)=0$, y las cargas del agujero negro

$$
Q_{A}=L^{2} r_{h} \frac{\mu_{A}}{g_{A}} \quad, \quad Q_{B}=L^{2} r_{h} \frac{\mu_{B}}{g_{B}}
$$

son ajustadas en términos de los potenciales químicos para tener campos de calibres que se anulen en el horizonte $A_{t}\left(r_{h}\right)=B_{t}\left(r_{h}\right)=0$, condiciṕon que garantiza una suave continuación euclídea ${ }^{24}$.La temperatura de Hawking es dada por,

$$
T=\frac{r_{h}{ }^{2} f^{\prime}\left(r_{h}\right)}{4 \pi l^{2}}=\frac{r_{h}}{4 \pi l^{2}}\left(3-\frac{Q_{A}{ }^{2}+Q_{B}{ }^{2}}{r_{h}{ }^{4}}\right)=\frac{r_{h}}{4 \pi L^{2}}\left(3-\frac{L^{4}}{r_{h}{ }^{2}}\left(\left(\frac{\mu_{A}}{g_{A}}\right)^{2}+\left(\frac{\mu_{B}}{g_{B}}\right)^{2}\right)\right)
$$

Los espinores $\Psi$ acoplados a esta geometría tendrán operadores fermiónicos duales en la teoría de campo que vive en el borde. Ellos deben satisfacer una ecuación de Dirac, en la cual los acoplamientos a los campos de calibre están parametrizados por diferentes cargas de prueba $q_{A}$ y $q_{B}$. En el apéndice presentamos un estudio de las ecuaciónes relevantes. Allí es mostrado que las soluciones en el espacio de momentos son de la forma,

$$
\Psi(x, z) \equiv f(r)^{-\frac{1}{4}}\left(\frac{L}{r}\right)^{\frac{3}{2}} e^{i k_{\mu} x^{\mu}} e^{i \theta S_{12}}\left(\begin{array}{l}
y_{\omega, k}(r) \\
z_{\omega, k}(r) \\
y_{\omega,-k}(r) \\
z_{\omega,-k}(r)
\end{array}\right)
$$

donde $y_{\omega, k}(r)$ está dado en (404), y $z_{\omega, k}(r)$ satisface la ecuación de Schrödinger (409) con potencial (410), explícitamente:

$$
V_{\omega, k}(s) \equiv \frac{1}{s_{0}^{2}(u(r)+k)^{2}}\left(k^{2}-u(r)^{2}-\frac{m}{L^{3}} r^{2} f(r)^{\frac{1}{2}}\left(\frac{r u^{\prime}(r)}{u(r)+k}-1\right)+\frac{m^{2}}{L^{2}} r^{2}\right)
$$

donde

$$
u(r)=f(r)^{-\frac{1}{2}}\left(\omega+\left(\mu_{A} q_{A}+\mu_{B} q_{B}\right)\left(1-\frac{r_{h}}{r}\right)\right)
$$

y $f(r)$ está dado en (365).

La siguiente observación resulta relevante. Si re-escaleamos los campos de calibre $A \rightarrow \alpha_{A} A, B \rightarrow \alpha_{B} B$, con parámetros arbitrarios $\alpha_{A}, \alpha_{B}$, y al mismo tiempo redefinimos las constantes de acoplamiento $g_{A} \rightarrow$ $\alpha_{A} g_{A}, g_{B} \rightarrow \alpha_{B} g_{B}$ y las cargas fermiónicas $q_{A} \rightarrow q_{A} / \alpha_{A}, q_{B} \rightarrow q_{B} / \alpha_{B}$, la acción (364)+(388) permanece invariante. Para cualquier solución que tiene al vacío de la geometría (365)-(366) en el UV, los campos de calibres re-escaleados imponen el re-escaleo de los potenciales químicos $\mu_{A} \rightarrow \alpha_{A} \mu_{A}, \mu_{B} \rightarrow \alpha_{B} \mu_{B}$. En particular podemos tomar $\alpha_{A}=g_{A}, \alpha_{B}=g_{B}$, es decir que normalizamos los campos de calibres de forma tal que: $g_{A}=g_{B}=1$; adoptaremos esta normalización en lo que sigue.

La teoría está definida por los cinco parámetros $\left(\mu_{A}, \mu_{B} ; q_{A}, q_{B} ; m\right)$. En lugar de los potenciales químicos será conveniente trabajar con el parámetro de dopaje ("doping") $\bar{x}$ y la temperatura (368),

$$
\bar{x}\left(\mu_{A}, \mu_{B}\right) \equiv \frac{\mu_{B}}{\mu_{A}} \quad ; \quad T\left(\mu_{A}, \mu_{B}\right)=T_{M}\left(1-\frac{3}{\left(4 \pi T_{M}\right)^{2}}\left(\mu_{A}^{2}+\mu_{B}{ }^{2}\right)\right)
$$

con expresiones inversas:

$$
\mu_{A}(\bar{x}, T)=\frac{4 \pi}{\sqrt{3}} \sqrt{\frac{T_{M}\left(T_{M}-T\right)}{1+\bar{x}^{2}}} \quad ; \quad \mu_{B}(\bar{x}, T)=\frac{4 \pi}{\sqrt{3}} \sqrt{T_{M}\left(T_{M}-T\right)} \frac{\bar{x}}{\sqrt{1+\bar{x}^{2}}}
$$

\footnotetext{
${ }^{24}$ Las constantes de acoplamiento adimensionales introducidas en [111] y la densidad de carga usual del agujero negro es definida por: $A_{t}(r)=\mu_{A}-\frac{\rho_{A}}{r}$ (lo mismo con $B_{t}(r)$ ), están relacionadas a las introducidas aquí por: $\lambda_{i}=\frac{\sqrt{2}}{g_{i}}, \rho_{i}=\frac{g_{i} Q_{i}}{L^{2}}$ respectivamente, $i=A, B$
} 
donde $T_{M} \equiv \frac{3 r_{h}}{4 \pi \mathrm{E}^{2}}$. Además introducimos el potencial efectivo y la carga efectiva por:

$$
\begin{aligned}
L^{2} r_{h} \mu_{\text {eff }} & \equiv Q_{\text {eff }} \equiv \sqrt{Q_{A}^{2}+Q_{B}^{2}}=L^{2} r_{h} \sqrt{\mu_{A}^{2}+\mu_{B}^{2}} \\
\mu_{\text {eff }} q_{\text {eff }} & \equiv \mu_{A} q_{A}+\mu_{B} q_{B}
\end{aligned}
$$

que en términos de $(\bar{x}, T)$ se escriben,

$$
\begin{aligned}
\mu_{\mathrm{eff}}(T) & =\frac{\sqrt{3} r_{h}}{L^{2}} \sqrt{1-\frac{T}{T_{M}}} \\
q_{\mathrm{eff}}\left(\bar{x} ; q_{A}, q_{B}\right) & =\frac{q_{A}+q_{B} \bar{x}}{\sqrt{1+\bar{x}^{2}}}
\end{aligned}
$$

Vemos que $\mu_{\text {eff }}(0)=\frac{\sqrt{3} r_{h}}{l^{2}}$ y tiende a cero para $T=T_{M}$. Por otro lado $q_{\text {eff }}$ interpola entre $q_{A}$ a $\bar{x}=0$ y $q_{B}$ como $\bar{x} \rightarrow \infty$, teniendo un máximo a $\bar{x}_{M}=\frac{q_{B}}{q_{A}}$.

El hecho importante es que el problema a mano resulta análogo al estudiado por Faulkner et al. en la referencia [109] si identificamos los parámetros $(Q, \mu, q)$ presentes allí con nuestros $\left(Q_{\text {eff }}, \mu_{\text {eff }}, q_{\text {eff }}\right)$ como son definidos en (374), (375). La función de Green está dada por la llamada "ecuación maestra " ecuación (54) en [109], la cual cerca de la superficie de Fermi $k=k_{F}$ luce como (ver ecuación (88) en [109] y (454))

$$
G_{R}(\omega, k) \sim H_{1}\left(\left|k-k_{F}\right|-\frac{\omega}{v_{F}}-H_{2} \omega^{2 \nu_{k_{F}}}\right)^{-1}
$$

donde las constantes $v_{F}, H_{1}$ and $H_{2}$ se leen de (454). El indice $\nu_{k}$ es dado por la ecuación (A31) en [109],

$$
\nu_{k}=\sqrt{\frac{m^{2} l^{2}}{6}+\frac{l^{4} k^{2}}{2 \sqrt{3} Q}-\frac{r_{h}^{2} l^{4} q^{2} \mu^{2}}{12 Q^{2}}}
$$

Notemos que $\nu_{k}$ en (376) debe ser evaluada en la FS $k=k_{F}$, donde a $T=0$, los $k_{F}$ 's son identificados como los polos de la función espectral a frecuencia nula, i.e. $\Im\left(G_{R}\left(0, k_{F}\right)\right)^{-1}=0$ (para $T>0$ tenemos que buscar el máximo de $\Im\left(G_{R}\left(0, k_{F}\right)\right.$ ). Ellos tienen que ser encontrados numéricamente resolviendo la ecuación de Dirac. Remarcamos que en nuestro contexto, $k_{F}=k_{F}\left(\bar{x}, T ; q_{A}, q_{B} ; m\right)$. Focalizaremos nuestra atención sobre la FS primaria, i.e. la FS con mas alto valor de $k_{F}$.

De lo dicho podemos tomar prestado el resultado (376); de (377) haciendo los reemplazos correspondientes el exponente de nuestro problema resulta:

$$
\nu_{k}=\sqrt{\frac{m^{2} l^{2}}{6}+\frac{l^{4} k^{2}}{2 \sqrt{3} Q_{\mathrm{eff}}}-\frac{r_{h}^{2} l^{4} q_{\mathrm{eff}}{ }^{2} \mu_{\mathrm{eff}}{ }^{2}}{12 Q_{\mathrm{eff}}{ }^{2}}}
$$

De las definiciónes de los parámetros efectivos (378) podemos escribir que,

$$
\nu_{k_{F}}\left(\bar{x}, T ; q_{A}, q_{B} ; m\right)=\sqrt{\frac{m^{2} l^{2}}{6}+\frac{l^{4} k_{F}\left(\bar{x}, T ; q_{A}, q_{B} ; m\right)^{2}}{6 r_{h}^{2} \sqrt{1-\frac{T}{T_{M}}}}-\frac{1}{12} \frac{\left(q_{A}+q_{B} \bar{x}\right)^{2}}{1+\bar{x}^{2}}}
$$

En el apéndice C3 revemos la derivación de la función de Green y la relevancia del índice $\nu_{k}$ para cualquier temperatura. El punto importante aquí es que, como vimos en el capítulo 4 el comportamiento metálico está directamente controlado por la carga del espinor a través de la cantidad $\nu_{k_{F}}$ que depende de esta. Para cargas tales que $\nu_{k_{F}}>1 / 2$, obtenemos el líquido de Fermi-Landau (excepto que el exponente de la frecuencia puede ser mucho mas grande que dos). Si la carga es tal que $\nu_{k_{F}}<1 / 2$ tenemos una superficie de Fermi con cuasi-partículas estables, pero en la cual la vida media de la cuasi-partícula escalea con la frecuencia de forma diferentes a lo esperado en un líquido de Landau. Finalmente, si la carga implica $\nu_{k_{F}}=1 / 2$, tenemos una superficie de Fermi pero cuyo escaleo con la frecuencia coincide exactamente a lo que se espera para las excitaciones que presenta un metal extraño.

Ya que para valores fijos de las cargas $\left(q_{A}, q_{B}\right)$ nuestro $\nu_{k_{F}}$ es una función de $(\bar{x}, T, m)$, conforme variamos el dopaje podemos en principio movernos del comportamiento típico de un líquido de Fermi-Landau a una región de metal extraño. El objetivo es entonces gráficar $\nu_{k_{F}}(\bar{x})$ como una función de $\bar{x}$, e identificar (si está presente) un cruce ("crossover") entre un líquido de Fermi-Landau a un metal extraño. Posteriormente se extiende el análisis con la inclusión de temperatura finita en nuestro esquema, siguiendo [110]. 


\subsection{Viendo el cruce a $T=0$ : resultados numéricos}

Para llevar a cabo los cálculos numéricos es conveniente usar la variable espinorial:

$$
\zeta_{\omega, k}(r) \equiv \frac{y_{\omega, k}(r)}{z_{\omega, k}(r)}
$$

De (403) la misma obedece la ecuación diferencial no lineal:

$$
\sqrt{\frac{g_{i i}}{g_{r r}}} \partial_{r} \zeta_{\omega, k}(r)=u_{\omega}(r)-k-2 m \sqrt{g_{i i}} \zeta_{\omega, k}(r)+\left(u_{\omega}(r)+k\right) \zeta_{\omega, k}(r)^{2}
$$

donde de (371),

$$
u_{\omega}(r)=f(r)^{-\frac{1}{2}}\left(\omega+\mu_{\mathrm{eff}}(T) q_{\mathrm{eff}}\left(\bar{x} ; q_{A}, q_{B}\right)\left(1-\frac{r_{h}}{r}\right)\right)
$$

La función $\zeta_{\omega, k}$ permite computar directamente la función de Green retardada utilizando la relación:

$$
G_{R}(\omega, k)=\lim _{r \rightarrow \infty} r^{m l} \zeta_{\omega, k}(r)
$$

que se deduce de las definiciones (417) y (418). Por otro lado, (381) debe ser resuelta con las b.c. en el IR dictadas por las b.c. entrantes impuestas sobre $y_{\omega, k}$ y $z_{\omega, k}$. Estas b.c se siguen de los siguientes comportamientos en el IR:

$\underline{\text { Caso } T=0} \underset{f}{\longrightarrow} \quad f(r)=\frac{f^{\prime \prime}\left(r_{h}\right)}{2}\left(r-r_{h}\right)^{2}+\ldots=\frac{L^{2}}{L_{2}^{2}} \rho^{2}(1+o(\rho))$

El bi-espinor (402) obedece:

$\psi_{\omega, k}^{(1)}(r) \stackrel{r \rightarrow r_{h}}{\longrightarrow} \begin{cases}a_{+}(0, k) v_{k}^{+} \rho^{-\nu_{k}}+\ldots+a_{-}(0, k) v_{k}^{-} \rho^{+\nu_{k}}+\left.\ldots\right|_{\rho=\frac{r}{r_{h}}-1} & , \quad \omega=0 \\ a_{+}(\omega, k)\left(\begin{array}{r}-i \\ 1\end{array}\right) e^{-i \frac{l_{2}^{2} \omega}{r_{h} \rho}}+\ldots+a_{-}(\omega, k)\left(\begin{array}{r}+i \\ 1\end{array}\right) e^{+i \frac{l_{2}{ }^{2} \omega}{r_{h} \rho}}+\left.\ldots\right|_{\rho=\frac{r}{r_{h}}-1} & \omega \neq 0\end{cases}$

donde $\nu_{k}$ es definido en (430), los espinores $v_{k}^{ \pm}$en (439), y $L_{2} \equiv \sqrt{\frac{2}{r_{h^{2} f^{\prime \prime}\left(r_{h}\right)}}} L$. Las b.c. entrantes implican:

$a_{+}(\omega, k)=0$, y por lo tanto $\left.\zeta_{\omega, k}(r)\right|_{\rho=0}=i$, que es la b.c. en el IR utilizada para resolver $(381)$.

En la figura (57) se muestran diferentes gráficos a $m$ y $q_{B}$ fijos, donde se grafica $\nu_{k_{F}}$ como función de la variable de dopaje para distintos valores de $q_{A}$. Se observa que en el límite $\bar{x} \rightarrow \infty$ todas las curvas confluyen a un mismo valor, como es de esperar dada la irelevancia del campo de calibre $A_{\mu}$ en este límite. Se aprecia además que para el rango de valores de $\bar{x}, \nu_{k_{F}}$ toma valores comprendidos entre cero y 1.5 aproximadamente. Esto nos indica que estamos recorriendo diferentes fases del sistema de acuerdo a si $\nu_{k_{F}}>1 / 2, \nu_{k_{F}}=1 / 2 \mathrm{y}$ $\nu_{k_{F}}<1 / 2$ como se explicó anteriormente. 

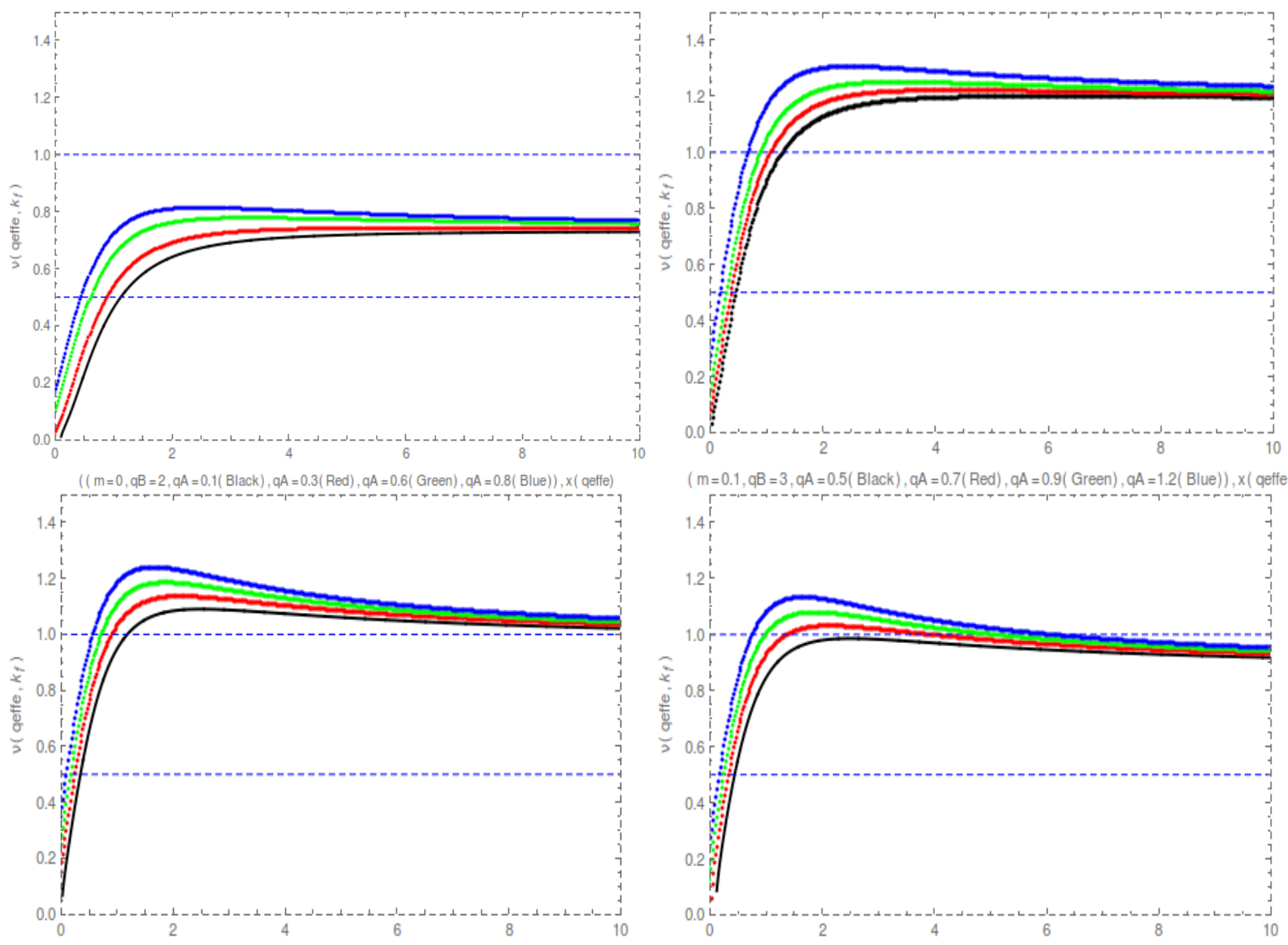

$(\mathrm{m}=0.3, \mathrm{qB}=3, \mathrm{qA}=1.2$ (Black), $\mathrm{qA}=1.4($ Red $), q \mathrm{~A}=1.6$ (Green), $\mathrm{qA}=1.8$ ( Blue) ); $\mathrm{x}(\mathrm{qeffe})$

$(m=0.4, q B=3, q A=1.2($ Black $), q A=1.4($ Red $), q A=1.6($ Green $), q A=1.8($ Blue $)) ; x$ (qeffe)

Figura 57: $\nu_{k_{F}}$ vs $x$ para diferentes valores de masas y cargas $q_{A}, q_{B}$ del fermión.

\subsection{Viendo el crossover a $T>0$ : resultados numéricos}

\subsection{Conclusiones}

\subsection{Apéndice C1: Convenciones}

Consideremos la métrica,

$$
g=g_{t t}(r) d t^{2}+g_{r r}(r) d r^{2}+g_{x x}(r) d x^{2}+g_{y y}(r) d y^{2}=\eta_{a b} \omega^{a} \omega^{b}
$$

donde elegimos los vierbein obvios y su base dual de vectores,

$$
\begin{aligned}
& \omega^{0} \equiv \sqrt{-g_{t t}(r)} d t \quad, \quad e_{0} \equiv \frac{1}{\sqrt{-g_{t t}(r)}} \frac{\partial}{\partial t} \\
& \omega^{1} \equiv \sqrt{g_{x x}(r)} d x \quad, \quad e_{1} \equiv \frac{1}{\sqrt{g_{x x}(r)}} \frac{\partial}{\partial x} \\
& \omega^{2} \equiv \sqrt{g_{y y}(r)} d y \quad, \quad e_{2} \equiv \frac{1}{\sqrt{g_{y y}(r)}} \frac{\partial}{\partial y} \\
& \omega^{3} \equiv \sqrt{g_{r r}(r)} d r \quad, \quad e_{3} \equiv \frac{1}{\sqrt{g_{r r}(r)}} \frac{\partial}{\partial r}
\end{aligned}
$$

Las componentes no nulas de la conexión de espin:

$$
\begin{aligned}
& \omega_{3}^{0}=-\omega_{03}=+\omega_{30}=e_{3}\left(\ln \sqrt{-g_{t t}(r)}\right) \omega^{0} \\
& \omega_{3}^{1}=+\omega_{13}=-\omega_{31}=e_{3}\left(\ln \sqrt{g_{x x}(r)}\right) \omega^{1} \\
& \omega_{3}^{2}=+\omega_{23}=-\omega_{32}=e_{3}\left(\ln \sqrt{g_{y y}(r)}\right) \omega^{2}
\end{aligned}
$$

La acción de un campo de Dirac acoplado a los campos de calibres $A$ y $B$ con cargas $q_{A}$ y $q_{B}$ respectivamente es,

$$
S^{(f e r)}=-\int d^{4} x \sqrt{-g} \bar{\Psi}\left(\Gamma^{a} D_{a}-m\right) \Psi \mp \int_{r \rightarrow \infty} d^{3} x \sqrt{-h} \bar{\Psi}_{ \pm} \Psi_{\mp},
$$


donde $\bar{\Psi} \equiv \Psi^{\dagger} i \Gamma^{0}, h=h_{\mu \nu}(x) d x^{\mu} d x^{\nu}$ es la métrica inducida en el borde $r \rightarrow \infty$, y la derivada covariante es definida por:

$$
D_{a} \Psi=\left(e_{a}+\frac{i}{2} \omega_{a}^{b c} S_{b c}-i q_{A} A_{a}-i q_{b} B_{a}\right) \Psi
$$

Las matrices gamma locales obedecen: $\left\{\Gamma^{a}, \Gamma^{b}\right\}=2 \eta^{a b}$ y $S_{a b} \equiv \frac{1}{4 i}\left[\Gamma_{a}, \Gamma_{b}\right]$ son los generadores en la representación espinorial del grupo local de Lorentz en $3+1$ dimensiones, y $\Psi_{ \pm} \equiv \frac{1}{2}\left(1 \mp \Gamma^{3}\right)$. El signo superior (inferior) en (305) corresponde a fijar $\Psi_{+}\left(\Psi_{-}\right)$en el borde; adoptaremos esta convención.

La ecuación de movimiento para el espinor es:

$$
\left(\Gamma^{a} D_{a}-m\right) \Psi=0
$$

En este trabajo consideramos el caso de invarianza rotacional, $g_{x x}=g_{y y}$ y asumimos que solo la componente temporal de los campos de calibres son no nulas. Para resolver (390) encontramos conveniente trabajar en espacio de momentos. Denotando $\left(x^{\mu}\right)=(t, x, y)$ y $\left(k_{\mu}\right)=\left(k_{t} \equiv-\omega, k_{x} \equiv k \cos \theta, k_{y} \equiv k \sin \theta\right)$, introducimos los bi-espinores $\left(\psi_{\omega, k}^{(\alpha)}(r), \alpha=1,2\right)$ como sigue:

$$
\Psi(x, z) \equiv\left(-g g^{r r}\right)^{-\frac{1}{4}} \int \frac{d k^{3}}{(2 \pi)^{3}} e^{i k_{\mu} x^{\mu}} e^{i \theta S_{12}}\left(\begin{array}{c}
\psi_{\omega, k}^{(1)}(r) \\
\psi_{\omega, k}^{(2)}(r)
\end{array}\right)
$$

También adoptamos una representación imaginaria pura para las matrices $\Gamma$ :

$$
\begin{aligned}
\Gamma^{0} \equiv\left(\begin{array}{cc}
i \sigma_{1} & 0 \\
0 & i \sigma_{1}
\end{array}\right) & \Gamma^{1} \equiv\left(\begin{array}{cc}
-\sigma_{2} & 0 \\
0 & \sigma_{2}
\end{array}\right) \\
\Gamma^{2} \equiv\left(\begin{array}{cc}
0 & \sigma_{2} \\
\sigma_{2} & 0
\end{array}\right) & \Gamma^{3} \equiv\left(\begin{array}{cc}
-\sigma_{3} & 0 \\
0 & -\sigma_{3}
\end{array}\right)
\end{aligned}
$$

De ecuación (390) se halla para $\psi_{\omega, k}^{(\alpha)}(r), \alpha=1,2$ :

$$
\left(\partial_{r}+m \sqrt{g_{r r}} \sigma_{3}\right) \psi_{\omega, k}^{(\alpha)}(r)=\sqrt{\frac{g_{r r}}{g_{x x}}}\left((-)^{\alpha} k \sigma_{1}+i u_{\omega}(r) \sigma_{2}\right) \psi_{\omega, k}^{(\alpha)}(r)
$$

donde

$$
u_{\omega}(r) \equiv \sqrt{-\frac{g_{x x}}{g_{t t}}}\left(\omega+q_{A} A_{t}+q_{B} B_{t}\right)
$$

Ya que de $(393)$ se sigue: $\psi_{\omega, k}^{(2)}(r) \propto \psi_{\omega,-k}^{(1)}(r)^{25}$, podemos enfocarnos en la ecuación $\alpha=1$

La prescripción AdS/CFT para computar la función de Green retardada

Cerca del borde de $A d S$, la solución general a (390) en $d+1$ dimensiones arbitrarias se comporta como:

$$
\Psi(x, r) \stackrel{r \rightarrow \infty}{\longrightarrow} r^{-\frac{d}{2}+m l} \psi_{+}(x)+\ldots+r^{-\frac{d}{2}-m l} \psi_{-}(x)+\ldots
$$

donde $\psi_{ \pm}(x)$ son la parte derecha y izquierda con respecto a $\Gamma^{d}, \Gamma^{d} \psi_{ \pm}(x)= \pm 1$. Si definimos el operador fermiónico de la QFT $\mathcal{O}$ dual a $\Psi$ tal que se acopla a la fuente $\psi_{+}$en la forma standard, $S^{(\text {source })}\left[\bar{\psi}_{+}, \psi_{+}\right]=$ $\int d x^{d}\left(\overline{\mathcal{O}}(x) \psi_{+}(x)+\bar{\psi}_{+}(x) \mathcal{O}(x)\right)$, entonces la prescripción AdS/CFT en el límite de un gran número de grados de libertad (límite de gran N) nos identifica la acción on-shell fermiónica en la geometría (305) con la función generatriz de las funciones de correlaciones conectadas en la teoría de campo, con lo cual:

$$
\left.G\left[\bar{\psi}_{+}, \psi_{+}\right] \equiv S^{(f e r)}\right|_{\text {on-shell }}=-\frac{1}{L^{d}} \int d x^{d} \bar{\psi}_{+}(x) \psi_{-}(x)
$$

donde en la ultima igualdad usamos (395) y la métrica $A d S_{d+1}$. La función de dos puntos en el espacio de momentos $\tilde{G}_{R}(k)$ es introducida por,

$$
-i<\tilde{\mathcal{O}}(k) \tilde{\mathcal{O}}^{\dagger}(p)>_{\text {conn }} \equiv(2 \pi)^{d} \delta^{d}(p-k) \tilde{G}_{R}(k)=C^{-1} \frac{\left.\delta^{2} S^{(f e r)}\right|_{\text {on-shell }}}{\delta \tilde{\psi}_{+}^{\dagger}(k) \delta \tilde{\psi}_{+}(p)} C^{-1}
$$

\footnotetext{
${ }^{25}$ Podemos ciertamente tomar una base de soluciones de (393) que verifica la igualdad; debido a la condiciones de contorno entrantes impuestas en el horizonte la solución que buscamos es única a menos de una normalización, y por lo tanto esta relación es cierta en nuestro contexto.
} 
La función de Green en la que estamos interesados se obtiene de (396) en la forma usual:

$$
\tilde{G}_{R}(k)=C^{-1} \int d p^{d}(2 \pi)^{d} \frac{\left.\delta^{2} S^{(f e r)}\right|_{\text {on-shell }}}{\delta \tilde{\psi}_{+}^{\dagger}(k) \delta \tilde{\psi}_{+}(p)} C^{-1}
$$

En nuestro caso de interés, $d=3$ y con las convenciones (392), (395) puede ser escrita:

$$
\left(\begin{array}{c}
\psi_{\omega, k}^{(1)}(r) \\
\psi_{\omega, k}^{(2)}(r)
\end{array}\right) \stackrel{r \rightarrow \infty}{\longrightarrow} r^{+m l}\left(\begin{array}{c}
0 \\
a^{(1)}(\omega, k) \\
0 \\
a^{(2)}(\omega, k)
\end{array}\right)+\ldots+r^{-m l}\left(\begin{array}{c}
b^{(1)}(\omega, k) \\
0 \\
b^{(2)}(\omega, k) \\
0
\end{array}\right)+\ldots
$$

con los $a^{(\alpha)}(\omega, k)$ definiendo la fuente arbitraria. Entonces la acción fermiónica resulta ser,

$$
\left.S^{(f e r)}\right|_{\text {on-shell }}=\int \frac{d k^{4}}{(2 \pi)^{4}}\left(a^{(1) *}(\omega, k) b^{(1)}(\omega, k)+a^{(2) *}(\omega, k) b^{(2)}(\omega, k)\right)
$$

entonces de (398) obtenemos,

$$
\tilde{G}_{R}(k)=\operatorname{diag}\left(G_{R}(\omega, k), 0, G_{R}(\omega,-k), 0\right) \quad, \quad b^{(\alpha)}(\omega, k) \equiv G_{R}\left(\omega,(-)^{1+\alpha} k\right) a^{(\alpha)}(\omega, k)
$$

Cabe mencionar que las b.c entrantes en el horizonte fijan completamente la solución para dadas $a^{(\alpha)}(\omega, k)$ no nulas; en vista de la linealidad de las ecuaciones, $b^{(\alpha)}(\omega, k) \propto a^{(\alpha)}(\omega, k)$ y por lo tanto el valor de la fuente es irrelevante para el cómputo de $\tilde{G}_{R}(k)$ en $(401)$.

\subsection{Apéndice C2: Una ecuación de Schrödinger para fermiones}

Si escribimos

$$
\psi_{\omega, k}^{(1)}(r) \equiv\left(\begin{array}{c}
y_{\omega, k}(r) \\
z_{\omega, k}(r)
\end{array}\right)
$$

entonces de (393) obtenemos un sistema de ecuaciones acopladas,

$$
\begin{aligned}
0 & =\left(\partial_{r}+m \sqrt{g_{r r}}\right) y_{\omega, k}(r)+\sqrt{\frac{g_{r r}}{g_{x x}}}\left(-u_{\omega}(r)+k\right) z_{\omega, k}(r) \\
0 & =\left(\partial_{r}-m \sqrt{g_{r r}}\right) z_{\omega, k}(r)+\sqrt{\frac{g_{r r}}{g_{x x}}}\left(+u_{\omega}(r)+k\right) y_{\omega, k}(r)
\end{aligned}
$$

De la segunda ecuación tenemos:

$$
y_{\omega, k}(r)=-\sqrt{\frac{g_{x x}}{g_{r r}}}\left(u_{\omega}(r)+k\right)^{-1}\left(\partial_{r}-m \sqrt{g_{r r}}\right) z_{\omega, k}(r)
$$

Remplazando en la primer ecuación obtenemos una ecuación de diferencial de segundo orden:

$$
z_{\omega, k}^{\prime \prime}(r)+P_{\omega, k}(r) z_{\omega, k}^{\prime}(r)+Q_{\omega, k}(r) z_{\omega, k}(r)=0
$$

donde

$$
\begin{aligned}
P_{\omega, k}(r) & =-\partial_{r} \ln \left(\sqrt{\frac{g_{r r}}{g_{x x}}}\left(u_{\omega}(r)+k\right)\right) \\
Q_{\omega, k}(r) & =-\frac{g_{r r}}{g_{x x}}\left(k^{2}-u_{\omega}(r)^{2}\right)+m \sqrt{g_{r r}} \partial_{r} \ln \left(\frac{u_{\omega}(r)+k}{\sqrt{g_{x x}}}\right)-m^{2} g_{r r}
\end{aligned}
$$

Haciendo el cambio de variables $r \rightarrow s(r)$, tal que:

$$
s^{\prime \prime}(r)+P_{\omega, k}(r) s^{\prime}(r)=0 \quad \longrightarrow \quad s^{\prime}(r)=s^{\prime}\left(r_{0}\right) e^{-\int_{r_{0}}^{r} d r^{\prime} P_{\omega, k}\left(r^{\prime}\right)}
$$

que con (406) conduce,

$$
s(r)=s_{0} \int_{\infty}^{r} d r^{\prime} \sqrt{\frac{g_{r r}}{g_{x x}}}\left(u_{\omega}(r)+k\right)
$$

donde $s_{0}$ es una constante arbitraria que pone la escala de $s$. Entonces (405) para $\left.\tilde{z}_{\omega, k}(s) \equiv z_{\omega, k}(r)\right|_{r(s)}$ toma la forma de una ecuación de Schrödinger:

$$
\tilde{z}_{\omega, k}^{\prime \prime}(s)-V_{\omega, k}(s) \tilde{z}_{\omega, k}(s)=0 \quad, \quad V_{\omega, k}(s) \equiv-\left.\frac{Q_{\omega, k}(r)}{s^{\prime}(r)^{2}}\right|_{r(s)}
$$

donde, explícitamente, el potencial efectivo es,

$$
\left.V_{\omega, k}(s) \equiv \frac{1}{s_{0}^{2}\left(u_{\omega}(r)+k\right)^{2}}\left(k^{2}-u_{\omega}(r)^{2}-m \frac{g_{x x}}{\sqrt{g_{r r}}} \partial_{r} \ln \left(\frac{u_{\omega}(r)+k}{\sqrt{g_{x x}}}\right)+m^{2} g_{x x}\right)\right|_{r(s)}
$$




\subsection{Apéndice C3: Funciones espectrales a baja energía}

Será conveniente en el análisis de esta sección rescribir las ecuaciones (403) introduciendo las componentes $y_{\omega, k}^{ \pm}$por medio de la siguiente rotación:

$$
\psi_{\omega, k}^{(1)}(r)=\left(\begin{array}{c}
y_{\omega, k}(r) \\
z_{\omega, k}(r)
\end{array}\right) \equiv \frac{1}{\sqrt{2}}\left(1-i \sigma_{1}\right)\left(\begin{array}{c}
y_{\omega, k}^{+}(r) \\
y_{\omega, k}^{-}(r)
\end{array}\right)=\left(\begin{array}{c}
\frac{1}{\sqrt{2}}\left(y_{\omega, k}^{+}(r)-i y_{\omega, k}^{-}(r)\right) \\
\frac{-i}{\sqrt{2}}\left(y_{\omega, k}^{+}(r)+i y_{\omega, k}^{-}(r)\right)
\end{array}\right)
$$

Las ecuaciónes (403) son ahora:

$$
\begin{aligned}
& 0=\left(\partial_{r}+i \sqrt{\frac{g_{r r}}{g_{x x}}} u_{\omega}(r)\right) y_{\omega, k}^{+}(r)+f_{k}^{+}(r) y_{\omega, k}^{-}(r) \\
& 0=\left(\partial_{r}-i \sqrt{\frac{g_{r r}}{g_{x x}}} u_{\omega}(r)\right) y_{\omega, k}^{-}(r)+f_{k}^{-}(r) y_{\omega, k}^{+}(r)
\end{aligned}
$$

donde hemos definido,

$$
f_{k}^{ \pm}(r) \equiv k \sqrt{\frac{g_{r r}}{g_{x x}}} \mp i m \sqrt{g_{r r}}
$$

De la primera/segunda ecuación en (412) podemos escribir $y_{\omega, k}^{\mp}(r)$ en términos $y_{\omega, k}^{ \pm}(r)$ como sigue,

$$
y_{\omega, k}^{\mp}(r)=-\frac{1}{f_{k}^{ \pm}(r)}\left(\partial_{r} \pm i \sqrt{\frac{g_{r r}}{g_{x x}}} u_{\omega}(r)\right) y_{\omega, k}^{ \pm}(r)
$$

Reemplazando en la segunda/primer ecuación, obtenemos ecuaciónes diferenciales de segundo orden,

$$
\partial_{r}^{2} y_{\omega, k}^{ \pm}(r)-\partial_{r} \ln f_{k}^{ \pm}(r) \partial_{r} y_{\omega, k}^{ \pm}(r)+q_{\omega, k}^{ \pm}(r) y_{\omega, k}^{ \pm}(r)=0
$$

donde,

$$
q_{\omega, k}^{ \pm}(r) \equiv \frac{g_{r r}}{g_{x x}}\left(u_{\omega}(r)^{2}-k^{2}\right)-m^{2} g_{r r} \pm i\left(\partial_{r}\left(\sqrt{\frac{g_{r r}}{g_{x x}}} u_{\omega}(r)\right)-\sqrt{\frac{g_{r r}}{g_{x x}}} u(r) \partial_{r} \ln f_{k}^{ \pm}(r)\right)
$$

Para obtener la función de Green retardada tenemos que resolver (415), imponer condiciones contorno entrantes en el horizonte, y aplicar la receta AdS/CMT. Esta tarea no es analíticamente posible en general y tenemos que pasar a los cálculos numéricos. No obstante en algunos casos como el que tenemos a mano donde el IR es esencialmente un agujero negro $A d S_{2}$, es posible obtener explícitamente el comportamiento a bajas frecuencias, y a bajas temperaturas usando el denominado "matching method" (método de unión o pegado), introducido muchos años atrás en el contexto de teoría de cuerdas [112]. Aquí seguiremos el procedimiento de [109] [110].

La idea es dividir el dominio de las coordenada $r$ en una región "interior" y una región "exterior", y unir ambas soluciones en una expansión a pequeñas frecuencias temperaturas (respecto del potencial químico $\mu$, la escala de la teoría). Con este fin, mientras el horizonte es definido como el punto $r_{h}$ tal que $f\left(r_{h}\right)=0$, también introducimos el punto $r_{*}<r_{h}$ tal que $f^{\prime}\left(r_{*}\right)=0$. De (368) es claro que ambos puntos coinciden a $T=0$.

\section{$\underline{\text { Región exterior }}$}

Es definida como la región donde $r_{*}+\frac{\sigma r_{h}}{\epsilon}<r<\infty$, donde $\sigma$ y $\epsilon$ son parámetros pequeños tales que en el límite $\sigma, \epsilon \rightarrow 0$,

$\sigma / \epsilon \rightarrow 0$. La región en el borde $r \rightarrow \infty$ se encuentra en esta región exterior, y la solución allí se comporta como:

$$
\psi_{\omega, k}^{(1)}(r) \stackrel{r \rightarrow \infty}{\longrightarrow} a(\omega, k) r^{m L}\left(\begin{array}{l}
0 \\
1
\end{array}\right)+\cdots+b(\omega, k) r^{-m L}\left(\begin{array}{l}
1 \\
0
\end{array}\right)+\ldots
$$

donde obviamos los supraíndices respecto del apéndice C.1. Fijando la fuente en el borde $\left(\begin{array}{l}0 \\ a(\omega, k)\end{array}\right)=$ $\lim _{r \rightarrow \infty} r^{-m L} \psi_{\omega, k}^{(1)}(r)$, la función de Green retardada de los operadores fermiónicos duales a $\psi_{\omega, k}^{(1)}$ es de acuerdo a (401) ${ }^{26}$ :

$$
G_{R}(\omega, k)=\frac{b(\omega, k)}{a(\omega, k)}
$$

\footnotetext{
${ }^{26}$ Cuando $0<m L<3 / 2$ es también posible fijar $b(\omega, k)$; aunque no consideraremos este caso directamente aquí.
} 
En general hay dos soluciones independientes a (393) (o a (415), (414)); vamos a llamar a ellas $\eta_{\omega, k}^{ \pm}(r)$ en la región exterior. Entonces podemos escribir,

$$
\psi_{\omega, k}^{(1)}(r)=\eta_{\omega, k}^{+}(r)+\mathcal{G}_{k}(\omega) \eta_{\omega, k}^{-}(r)
$$

donde $\mathcal{G}_{k}(\omega)$ es la normalización relativa.

Dado que en la región exterior la ecuación (415) es bien comportada alrededor de $\omega=0$, podemos elegir ambas $\eta_{\omega, k}^{ \pm}(r)$ holomorfas en $\omega$ y escribir una expansión:

$$
\psi_{\omega, k}^{(1)}(r)=\sum_{p=0}^{\infty}\left(\eta_{p, k}^{+}(r)+\mathcal{G}_{k}(\omega) \eta_{p, k}^{-}(r)\right) \omega^{p}
$$

Las funciónes $\eta_{m, k}^{ \pm}(r)$ son calculadas resolviendo el conjunto (infinito) de ecuaciónes derivadas de (415) orden a orden en $\omega$. Ellas presentan cerca del borde un comportamiento similar a (417),

$$
\eta_{p, k}^{ \pm}(r) \stackrel{r \rightarrow \infty}{\longrightarrow} a_{p}^{ \pm}(k) r^{m L}\left(\begin{array}{c}
0 \\
1
\end{array}\right)+\cdots+b_{p}^{ \pm}(k) r^{-m L}\left(\begin{array}{c}
1 \\
0
\end{array}\right)+\ldots
$$

entonces de (417), (420), (418) podemos escribir,

$$
G_{R}(\omega, k)=\frac{b^{+}(\omega, k)+\mathcal{G}_{k}(\omega) b^{-}(\omega, k)}{a^{+}(\omega, k)+\mathcal{G}_{k}(\omega) a^{-}(\omega, k)}
$$

donde hemos definido los coeficientes holomórficos:

$$
a^{ \pm}(\omega, k)=\sum_{p=0}^{\infty} a_{p}^{ \pm}(k) \omega^{p} \quad ; \quad b^{ \pm}(\omega, k)=\sum_{p=0}^{\infty} b_{p}^{ \pm}(k) \omega^{p}
$$

Enfatizamos que ellos están determinados unívocamente orden por orden en $\omega$ a través de (421) una vez que las funciónes $\eta_{\omega, k}^{ \pm}(r)$ están completamente definidas.

Región interior

El problema de computar (418) depende de la conexión del comportamiento en el UV (417) con la solución en el IR. Si la solución en el IR es conocida ( y este es el punto crucial), el procedimiento de matching (pegado) que brevemente explicamos debajo permite computar (418) en el régimen de frecuencias y temperaturas bajas.

La región interior es definida introduciendo las coordenadas $\xi$ y $\tau$ por:

$$
\begin{aligned}
r & \equiv r_{*}+\frac{\sigma r_{h}}{\xi} \leftrightarrow \xi \equiv \frac{\sigma r_{h}}{r-r_{*}} \quad, \quad \epsilon<\xi<\xi_{h} \\
\tau & \equiv \frac{L \sigma}{L_{2}{ }^{2}} t
\end{aligned}
$$

donde mantenemos el parámetro: $\xi_{h} \equiv \sigma\left(1-\frac{r_{*}}{r_{h}}\right)^{-1}$ fijo, y definimos $L_{2}$ vía:

$$
\frac{L^{2}}{L_{2}^{2}} \equiv \frac{r_{h}^{2} f^{\prime \prime}\left(r_{h}\right)}{2}=d(d-1)
$$

donde la última igualdad es válida para la solución RNAdSBH. En términos de estas coordenadas tenemos que:

$$
f(r)=\sigma^{2} \frac{L^{2}}{L_{2}^{2}} \frac{\tilde{f}\left(\frac{\xi}{\xi_{h}}\right)}{\xi^{2}}(1+o(\sigma)) \quad, \quad \tilde{f}(x) \equiv 1-x^{2}
$$

Los campos toman la forma:

$$
\begin{aligned}
& g=\frac{L_{2}^{2}}{\xi^{2}}\left(-\tilde{f}\left(\frac{\xi}{\xi_{h}}\right) d \tau^{2}+\frac{d \xi^{2}}{\tilde{f}\left(\frac{\xi}{\xi_{h}}\right)}\right)+\frac{r_{h}^{2}}{L^{2}}\left(d x^{2}+d y^{2}\right)+o(\sigma) \\
& A=d \tau \frac{e_{3}}{\xi}\left(1-\frac{\xi}{\xi_{h}}\right)+o(\sigma) \quad, \quad e_{3} \equiv \frac{\mu L_{2}^{2}}{L}
\end{aligned}
$$


Podemos reconocer los términos a primer orden en $\sigma$ como la solución de agujero negro $A d S_{2} \times \Re^{2}$. Con respecto a $\tau$, la temperatura de este agujero negro es $T_{\tau}=\frac{1}{2 \pi \xi_{h}}$; junto con la frecuencia definida por $\omega t \equiv \omega_{\tau} \tau$ ellos están relacionados a la original vía,

$$
T=\sigma \frac{r_{h}}{L_{2}^{2}} T_{\tau} \quad ; \quad \omega \equiv \sigma \frac{L}{L_{2}^{2}} \omega_{\tau}
$$

Estas relaciones muestran que en el límite $\sigma \rightarrow 0$ para un valor fijo de $\omega_{\tau}$ y $T_{\tau}, \omega$ y $T$ tiende a cero mientras que $\frac{\omega}{T}$ permanece fija. Estamos interesados en la expansión a bajas frecuencia y temperatura, entonces a primer orden en $\sigma$, la ecuación fermiónica (415) se convierte en,

$$
\begin{aligned}
0 & =\left(\xi^{2} \partial_{\xi}^{2}+\left(2-\frac{1}{\tilde{f}\left(\frac{\xi}{\xi_{h}}\right)}\right) \xi \partial_{\xi}+v_{\omega, k}^{ \pm}\left(\frac{\xi}{\xi_{h}}\right)\right) y_{\omega, k}^{ \pm}(r) \\
v_{\omega, k}^{ \pm}(x) & \equiv \frac{x^{2}}{\tilde{f}(x)^{2}}\left(W \xi_{h}+E_{3}\left(\frac{1}{x}-1\right)\right)^{2}-\frac{\hat{K}^{2}+\hat{M}^{2}}{\tilde{f}(x)} \mp i \frac{x}{\tilde{f}(x)^{2}}\left(W \xi_{h}-E_{3}(1-x)\right)
\end{aligned}
$$

donde hemos introducido $W=\frac{L}{r_{h}} \omega_{\tau}$ y las tres constantes adimensionales: $\hat{K} \equiv \frac{L L_{2}}{r_{h}} k, \hat{M} \equiv m L_{2} \mathrm{y}$ $E_{3} \equiv \frac{L}{r_{h}} e_{3}=\frac{L_{2}^{2}}{r_{h}} \mu$. En términos de ellas también introducimos el índice,

$$
\nu_{k} 2 \equiv \hat{K}^{2}+\hat{M}^{2}-E_{3}^{2}
$$

Caso $T=0, \xi_{h} \rightarrow \infty$

Definimos las siguientes funciones:

$$
W_{ \pm}(\xi ; \nu, W, E) \equiv(i 2 W \xi)^{-\frac{1}{2}} W_{\mp \frac{1}{2}-i E, \nu}(i 2 W \xi)
$$

donde $W_{\lambda \nu}(z)$ es la función de Whittaker. Para $\xi \rightarrow \epsilon \rightarrow 0^{+}$y $\xi \rightarrow \infty$ tiene el siguiente comportamiento:

$$
\begin{aligned}
& W_{ \pm}(\xi ; \nu, W, E) \stackrel{\xi \rightarrow 0^{+}}{\longrightarrow} \frac{\Gamma(-2 \nu)}{\Gamma\left(\frac{1}{2} \pm \frac{1}{2}-\nu+i E\right)}(i 2 W \xi)^{\nu}+\cdots+\frac{\Gamma(2 \nu)}{\Gamma\left(\frac{1}{2} \pm \frac{1}{2}+\nu+i E\right)}(i 2 W \xi)^{-\nu}+\ldots \\
& W_{ \pm}(\xi ; \nu, W, E) \stackrel{\xi \rightarrow \infty}{\longrightarrow}(i 2 W \xi)^{-\frac{1}{2} \mp \frac{1}{2}-i E} e^{-i W \xi}\left(1+o\left(\frac{1}{\xi}\right)\right)
\end{aligned}
$$

Entonces la solución a (429) puede ser escrita como,

$$
y_{\omega, k}^{ \pm}(r)=A_{\omega, k}^{ \pm} W_{ \pm}\left(\xi ; \nu_{k}, W, E_{3}\right)+B_{\omega, k}^{ \pm} W_{\mp}\left(\xi ; \nu_{k}, W, E_{3}\right)
$$

Pero de la segunda línea en (432) y ya que los términos $e^{\mp i W \xi}$ son ondas salientes/entrantes cerca del horizonte $\xi \rightarrow \infty$, la imposición de las b.c entrantes implica que: $A_{\omega, k}^{ \pm}=0$. Entonces usando la primer ecuación en (432) obtenemos en el borde del agujero negro $A d S_{2}$ :

$$
y_{\omega, k}^{ \pm}(r) \stackrel{\xi \rightarrow 0^{+}}{\longrightarrow} B_{\omega, k}^{ \pm}\left(\frac{\Gamma\left(-2 \nu_{k}\right)}{\Gamma\left(\frac{1}{2} \mp \frac{1}{2}-\nu-i E_{3}\right)}(-i 2 W \xi)^{\nu}+\cdots+\frac{\Gamma\left(2 \nu_{k}\right)}{\Gamma\left(\frac{1}{2} \mp \frac{1}{2}+\nu-i E_{3}\right)}(-i 2 W \xi)^{-\nu}+\ldots\right)
$$

No obstante $y_{\omega, k}^{ \pm}(r)$ no son independientes debido a las relaciones (414), que en las variables interiores se escriben como:

$$
y_{\omega, k}^{ \pm}(r)=\frac{1}{\hat{K} \pm i \hat{M}}\left(\xi \partial_{\xi} y_{\omega, k}^{\mp}(r) \pm i\left(E_{3}+W_{\tau} \xi\right) y_{\omega, k}^{\mp}(r)\right)
$$

En particular del comportamiento de la solución para $\xi$ yendo a cero (434), ecuaciones (435) implican,

$$
A_{\omega, k}^{+}=-(\hat{K}-i \hat{M}) A_{\omega, k}^{-} \quad ; \quad B_{\omega, k}^{-}=-(\hat{K}+i \hat{M}) B_{\omega, k}^{+}
$$

las cuales determinan, i.e, $y_{\omega, k}^{-}(r)$ en términos de $y_{\omega, k}^{+}(r)$. Con la ayuda de (436), de (411) obtenemos:

$$
\begin{aligned}
\psi_{\omega, k}^{(1)}(r) \stackrel{\xi \rightarrow 0^{+}}{\longrightarrow} & -\frac{B_{\omega, k}^{+}}{\sqrt{2}}\left(\Gamma\left(2 \nu_{k}\right)\left(\begin{array}{l}
\frac{-\nu_{k}+\hat{M}+i\left(E_{3}-\hat{K}\right)}{\Gamma\left(1+\nu_{k}-i E_{3}\right)} \\
\frac{E_{3}+\hat{K}+i\left(\nu_{k}+\hat{M}\right)}{\Gamma\left(1-\nu_{k}+i E_{3}\right)}
\end{array}\right)(-i 2 W \xi)^{-\nu_{k}}+\ldots\right. \\
& \left.+\quad \Gamma\left(-2 \nu_{k}\right)\left(\begin{array}{l}
\frac{\nu_{k}+\hat{M}+i\left(E_{3}-\hat{K}\right)}{\Gamma\left(1-\nu_{k}-i E_{3}\right)} \\
\frac{E_{3}+\hat{K}+i\left(-\nu_{k}+\hat{M}\right)}{\Gamma\left(1+\nu_{k}+i E_{3}\right)}
\end{array}\right)(-i 2 W \xi)^{\nu_{k}}+\ldots\right)
\end{aligned}
$$


que en forma abreviada se puede escribir como:

$$
\psi_{\omega, k}^{(1)}(r) \stackrel{\xi \rightarrow 0^{+}}{\longrightarrow} a_{\omega, k}\left(\frac{L_{2}^{2}}{r_{h} \sigma} \xi\right)^{-\nu_{k}} v_{k}^{-}+b_{\omega, k}\left(\frac{L_{2}^{2}}{r_{h} \sigma} \xi\right)^{\nu_{k}} v_{k}^{+}
$$

donde hemos introducido los espinores,

$$
v_{k}^{ \pm} \equiv\left(\begin{array}{c} 
\pm \nu_{k}+\hat{M} \\
\hat{K}+E_{3}
\end{array}\right)
$$

y las constantes,

$$
\begin{aligned}
& a_{\omega, k}=-(-i 2 \omega)^{-\nu_{k}} \frac{\Gamma\left(2 \nu_{k}\right)}{\Gamma\left(1+\nu_{k}-i E_{3}\right)}\left(1+i \frac{\nu_{k}+\hat{M}}{\hat{K}+E_{3}}\right) \frac{B_{\omega, k}^{+}}{\sqrt{2}} \\
& b_{\omega, k}=-(-i 2 \omega)^{\nu_{k}} \frac{\Gamma\left(-2 \nu_{k}\right)}{\Gamma\left(1-\nu_{k}-i E_{3}\right)}\left(1+i \frac{-\nu_{k}+\hat{M}}{\hat{K}+E_{3}}\right) \frac{B_{\omega, k}^{+}}{\sqrt{2}}
\end{aligned}
$$

De acuerdo a (401) se puede introducir la función de Green en dos dimensiones del operador fermiónico dual definido por la fuente $a_{\omega, k}$ en (438):

$$
\mathcal{G}_{k}^{(2)}(\omega) \equiv \frac{b_{\omega, k}}{a_{\omega, k}}=e^{-i \pi \nu_{k}} \frac{\Gamma\left(-2 \nu_{k}\right)}{\Gamma\left(2 \nu_{k}\right)} \frac{\Gamma\left(1+\nu_{k}-i E_{3}\right)}{\Gamma\left(1-\nu_{k}-i E_{3}\right)} \frac{\nu_{k}+\hat{M}+i\left(E_{3}+\hat{K}\right)}{-\nu_{k}+\hat{M}+i\left(E_{3}-\hat{K}\right)}(2 \omega)^{2 \nu_{k}}
$$

Caso $T>0, \xi_{h}<\infty$

Definimos la siguiente función:

$$
F(x ; \nu, W, E) \equiv\left(\frac{1-x}{1+x}\right)^{-i\left(\frac{W}{2}-E\right)}\left(\frac{2 x}{1-x}\right)^{\nu}{ }_{2} F_{1}\left(\nu-i E, \frac{1}{2}+\nu+i(W-E) ; 1+2 \nu ;-\frac{2 x}{1-x}\right)
$$

donde ${ }_{2} F_{1}(\alpha, \beta ; \gamma ; z)$ es la función hipergeométrica. Presenta los siguientes comportamientos:

$$
\begin{array}{rll}
F(x ; \nu, W, E) & \stackrel{x \rightarrow 0^{+}}{\longrightarrow} & (2 x)^{\nu}+\ldots \\
F(x ; \nu, W, E) & \stackrel{x \rightarrow 1^{-}}{\longrightarrow} & \frac{\Gamma(1+2 \nu) \Gamma\left(\frac{1}{2}+i W\right)}{\Gamma\left(\frac{1}{2}+\nu+i(W-E)\right) \Gamma(1+\nu+i E)}\left(\frac{1-x}{2}\right)^{-i \frac{W}{2}}+\ldots \\
& + & \frac{\Gamma(1+2 \nu) \Gamma\left(-\frac{1}{2}-i W\right)}{\Gamma(\nu-i E) \Gamma\left(\frac{1}{2}+\nu-i(W-E)\right)}\left(\frac{1-x}{2}\right)^{\frac{1}{2}+i \frac{W}{2}}+\ldots
\end{array}
$$

Entonces la solución a (429) puede ser escrita como,

$$
y_{\omega, k}^{ \pm}(r)=A_{\omega, k}^{ \pm} F\left(\frac{\xi}{\xi_{h}} ;-\nu_{k}, \pm W_{\tau}, \pm E_{3}\right)+B_{\omega, k}^{ \pm} F\left(\frac{\xi}{\xi_{h}} ;+\nu_{k}, \pm W_{\tau}, \pm E_{3}\right)
$$

Usando la primer ecuación en (443) obtenemos en el borde del agujero negro $A d S_{2}, \xi \rightarrow \epsilon \rightarrow 0^{+}$:

$$
y_{\omega, k}^{ \pm}(r) \stackrel{\xi \rightarrow 0^{+}}{\longrightarrow} A_{\omega, k}^{ \pm}\left(2 \frac{\xi}{\xi_{h}}\right)^{-\nu_{k}}+\cdots+B_{\omega, k}^{ \pm}\left(2 \frac{\xi}{\xi_{h}}\right)^{+\nu_{k}}+\ldots
$$

No obstante $y_{\omega, k}^{ \pm}(r)$ no son independientes debido a las relaciones (414), que en las variables interiores se escriben como:

$$
y_{\omega, k}^{ \pm}(r)=\frac{1}{\hat{K} \pm i \hat{M}}\left(\tilde{f}(\xi) \xi \partial_{\xi} y_{\omega, k}^{\mp}(r) \pm i\left(E_{3}+\left(W-\frac{E_{3}}{\xi_{h}}\right) \xi\right) y_{\omega, k}^{\mp}(r)\right)
$$

En particular, del comportamiento para grandes valores de $\xi(445)$ obtenemos que:

$$
A_{\omega, k}^{-}=\frac{\hat{K}+i \hat{M}}{-\nu_{k}+i E_{3}} A_{\omega, k}^{+} \quad ; \quad B_{\omega, k}^{-}=\frac{\hat{K}+i \hat{M}}{+\nu_{k}+i E_{3}} B_{\omega, k}^{+}
$$


la cual determina, i.e., $y_{\omega, k}^{-}(r)$ en términos de $y_{\omega, k}^{+}(r)$.

Por otro lado, para obtener el comportamiento cerca del horizonte debemos usar la segunda ecuación en (443). Los términos con la dependencia $(1-x)^{-i \frac{W_{\tau}}{2}}$ corresponden a ondas entrantes mientras que aquellos con $(1-x)^{+i \frac{W_{\tau}}{2}}$ son ondas salientes $\left(x=\xi / x i_{h}\right)$. Imponiendo b.c entrantes estos últimos términos se cancelan, y nos conducen a las relaciones entre coeficientes:

$$
\begin{aligned}
& \frac{A_{\omega, k}^{+}}{B_{\omega, k}^{+}}=\frac{\Gamma\left(+2 \nu_{k}\right)}{\Gamma\left(-2 \nu_{k}\right)} \frac{\Gamma\left(-\nu_{k}-i E_{3}\right)}{\Gamma\left(+\nu_{k}-i E_{3}\right)} \frac{\Gamma\left(\frac{1}{2}-\nu_{k}-i\left(W-E_{3}\right)\right)}{\Gamma\left(\frac{1}{2}+\nu_{k}-i\left(W-E_{3}\right)\right)} \\
& \frac{A_{\omega, k}^{-}}{B_{\omega, k}^{-}}=\frac{\Gamma\left(+2 \nu_{k}\right)}{\Gamma\left(-2 \nu_{k}\right)} \frac{\Gamma\left(1-\nu_{k}-i E_{3}\right)}{\Gamma\left(1+\nu_{k}-i E_{3}\right)} \frac{\Gamma\left(\frac{1}{2}-\nu_{k}-i\left(W-E_{3}\right)\right)}{\Gamma\left(\frac{1}{2}+\nu_{k}-i\left(W-E_{3}\right)\right)}
\end{aligned}
$$

Es directo chequear la compatibilidad entre (447) y (448). Con la ayuda de (447), de (411) tenemos:

$$
\begin{gathered}
\psi_{\omega, k}^{(1)}(r) \stackrel{\xi \rightarrow 0^{+}}{\longrightarrow} \frac{A_{\omega, k}^{+}}{\sqrt{2}\left(-\nu_{k}+i E_{3}\right)}\left(\begin{array}{c}
-\nu_{k}+\hat{M}+i\left(E_{3}-\hat{K}\right) \\
E_{3}+\hat{K}+i\left(\nu_{k}+\hat{M}\right)
\end{array}\right)\left(\frac{2 \xi}{\xi_{h}}\right)^{-\nu_{k}}+\ldots \\
+\frac{B_{\omega, k}^{+}}{\sqrt{2}\left(\nu_{k}+i E_{3}\right)}\left(\begin{array}{c}
\nu_{k}+\hat{M}+i\left(E_{3}-\hat{K}\right) \\
E_{3}+\hat{K}+i\left(-\nu_{k}+\hat{M}\right)
\end{array}\right)\left(\frac{2 \xi}{\xi_{h}}\right)^{\nu_{k}}+\ldots
\end{gathered}
$$

con $A_{\omega, k}^{+}$y $B_{\omega, k}^{+}$relacionados por la primera ecuación en (448). Una expansión análoga a (437) permite introducir la versión de (441) a temperatura finita, con:

$$
\begin{aligned}
a_{\omega, k} & =-\frac{(4 \pi T)^{-\nu_{k}}}{-\nu_{k}+i E_{3}}\left(1+i \frac{\nu_{k}+\hat{M}}{\hat{K}+E_{3}}\right) \frac{A_{\omega, k}^{+}}{\sqrt{2}} \\
b_{\omega, k} & =-\frac{(4 \pi T)^{\nu_{k}}}{\nu_{k}+i E_{3}}\left(1+i \frac{-\nu_{k}+\hat{M}}{\hat{K}+E_{3}}\right) \frac{B_{\omega, k}^{+}}{\sqrt{2}}
\end{aligned}
$$

El procedimiento de pegado

Para determinar completamente la solución fermiónica, tenemos que unir la solución en el interior $\xi=$ $\epsilon \rightarrow 0^{+}$con la solución en el exterior en $r=r_{*}+\frac{\sigma r_{h}}{\epsilon}$. El camino más directo para hacerlo es el siguiente. Escribamos el comportamiento a primer orden de la solución en el interior en la forma:

$$
\psi_{\omega, k}^{(1)}(r) \stackrel{\xi \rightarrow 0^{+}}{\longrightarrow} a_{\omega, k} v_{k}^{-}\left(\frac{L_{2}^{2}}{r_{h} \sigma} \xi\right)^{-\nu_{k}}+\cdots+\mathcal{G}_{k}^{(2)}(\omega) a_{\omega, k} v_{k}^{+}\left(\frac{L_{2}^{2}}{r_{h} \sigma} \xi\right)^{\nu_{k}}+\ldots
$$

Ahora consideremos el primer orden de la solución exterior, término $p=0$ en (420). No es difícil ver que las ecuaciónes verificadas por las funciónes $\eta_{0, k}^{ \pm}(r)$ son exactamente las mismas que las ecuaciones verificadas por la solución interior en el límite $\xi \rightarrow 0^{+}$. Por lo tanto, si definimos $\eta_{0, k}^{ \pm}(r)$ imponiendo en el IR el siguiente comportamiento:

$$
\eta_{0, k}^{ \pm}(r) \stackrel{r \rightarrow r_{h}}{\longrightarrow} a_{\omega, k} v_{k}^{\mp}\left(\frac{r-r_{h}}{L_{2}{ }^{2}}\right)^{ \pm \nu_{k}}+\ldots
$$

entonces el pegado es automáticamente realizado con la identificación de la normalización relativa $\mathcal{G}_{k}(\omega)$ en (419) con la función de Green retardada de la CFT dual a la geometría $A d S_{2} \times \Re^{2}, \mathcal{G}_{k}^{(2)}(\omega)$ introducida en (441). Llevando a cabo las identificaciones, tendremos:

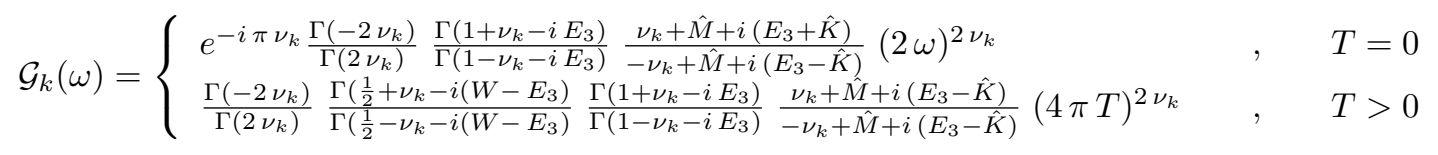

Por lo tanto la función de Green (422) a bajas frecuencias resulta:

$$
G_{R}(\omega, k)=\frac{b_{0}^{+}(k)+b_{1}^{+}(k) \omega+\cdots+\left(b_{0}^{-}(k)+\ldots\right) \mathcal{G}_{k}(\omega)}{a_{0}^{+}(k)+a_{1}^{+}(k) \omega+\cdots+\left(a_{0}^{-}(k)+\ldots\right) \mathcal{G}_{k}(\omega)}
$$




\title{
8 Inestabilidades de Pomeranchuk holográficas
}

\begin{abstract}
Resumen
Usando la descripción holográfica de la fase de metal extraño, desarrollaremos un método para detectar inestabilidades fermiónicas que conducen a una fase nemática. La propuesta es usar la técnica de Pomeranchuk sobre fermiones débilmente acoplados en un espacio-tiempo curvo, como señal de inestabilidades anisotrópicas de la teoría fuertemente acoplada que vive en el borde. Exploramos el diagrama de fase a temperatura cero para un valor finito del potencial químico en el borde, variando un acoplamiento femiónico cuártico.
\end{abstract}

\subsection{Introducción}

Ha sido propuesto que la transición de una fase normal de líquido de Fermi a una fase nemática es inducida vía una inestabilidad de Pomeranchuk [3]. Tales inestabilidades surgen cuando un líquido de Fermi es perturbado con una exitación fermiónica, representada por una deformación anisotrópica de su superficie de Fermi, que resulta en un decrecimiento neto de la energía total. Descomponiendo la deformación de la superficie de Fermi en una base de armónicos esféricos, e insertando dicha descomposición en la fórmula de Landau para la energía de una excitación, Pomeranchuk fue capaz de escribir un conjunto de condiciones bajo las cuales un líquido de Fermi es estable. Su método puede ser generalizado a sistemas con redes o a superficies de Fermi anisotrópicas [111]; dicha posibilidad depende de la debilidad del acoplamiento entre las cuasi-partículas, o en otras palabras de la validez de la fórmula de Landau. Esto implica que desde la perspectiva de la fase de metal extraño, ya que la dinámica es fuertemente acoplada, la detección de inestabilidades fermiónicas se hace más difícil.

Recientemente ha sido mostrado que la descripción holográfica de una fase fermiónica homogénea cuenta con algunas propiedades interesantes del metal extraño [109]-[118]. En particular, la función espectral resultante es compatible con una superficie de Fermi con cuasi-partículas de gran vida media. Basándonos en eso, en este artículo, analizamos las inestabilidades de Pomeranchuk de la fase de metal extraño desde la perspectiva holográfica. Usaremos la geometría del espacio-tiempo de una "estrella de electrones" dentro en la cual propagaremos un campo de Dirac acoplado débilmente [115]-[118]. Asumiendo que los campos en el espacio-tiempo curvado interactúan débilmente, pueden ser descriptos por una teoría de Landau en la aproximación de campo de medio, y por lo tanto podemos estudiar sus inestabilidades bajo la deformación de la superficie de Fermi. Esto nos permite tener en cuenta las inestabilidades anisotrópicas del metal extraño dual.

\subsection{Marco general}

El método de Pomeranchuk para detectar inestabilidades fermiónicas en un espacio-tiempo de $2+1$ dimensiones depende de la representación en el espacio de momentos del sistema fermiónico (ver apéndice 8.6 para una introducción). Usamos la teoría de Landau de líquido de Fermi, que depende de una "relación de dispersión renormalizada" y una "función de interacción de cuasi-partículas" las cuales son obtenidas del Hamiltoniano perturbativo o de cálculos de campo medio. Este procedimiento es en general seguro solo en el límite de acoplamiento débil, ya que la fase de metal extraño se supone que es una fase fuertemente acoplada, el único camino para aplicar el método de Pomeranchuk sería por medio de una descripción de acoplamiento débil. La holografía provee tal dual, el precio a pagar es añadir una dimensión extra teniendo así un espacio-tiempo curvo de $3+1$ dimensiones.

La generalización del método a un espacio curvo arbitrario no es posible, debido a la ausencia de una representación en el espacio de momentos. De todas maneras el tipo especial de geometría usada en holografía tiene la característica adicional de simetría traslacional en las dos direcciones espaciales que abarcan el borde. Esto permite etiquetar los estados en el espacio-tiempo curvo con un índice de momento 2-dimensional $\vec{k}$. A tal índice se le agrega un índice adicional $n$ que etiqueta el modo de oscilación sobre la dirección holográfica. Esto implica que el espacio curvo de $3+1$ dimensiones del sistema de fermiones puede ser entendido como un sistema en un espacio plano de dimensión $2+1$ con muchos fermiones etiquetados por el índice $n$. En conclusión, el método de Pomeranchuk puede ser extendido a un sistema de muchos fermiones acoplados débilmente en un espacio plano.

En lo que sigue esbozaremos los pasos necesarios para ir de una acción para espinores en un espacio-tiempo asintóticamente AdS a un espacio-tiempo plano de $2+1$ dimensiones para un sistema de múltiples fermiones. Luego construiremos la teoría de Landau relevante para tal sistema y aplicaremos el método de Pomeranchuk a éste. La acción en un espacio curvo en $3+1$ dimensiones y la métrica de fondo serán presentadas en la sección 8.3. Las etiquetas para los estados fermiónicos en tal espacio corresponden al momento en dos dimensiones, las especies fermiónicas y el espín, son introducidas en la sección 8.4, en la cual la ecuación de Dirac libre 
es resuelta en la aproximación WKB. Finalmente en la sección 8.5 el Hamiltoniano resultante en $2+1$ dimensiones es presentado, y partir de éste la interacción de Landau y la relación de dispersión normalizada son obtenidas vía un calculo de campo medio. Con estos elementos, la construcción de Pomeranchuk es imitada, resultando en un conjunto de condiciones de estabilidad. Evaluando tales condiciones para la interacción (458), se espera que en alguna región del espacio de constantes de acoplamiento se viole alguna de las desigualdades de Pomeranchuk, originando una superficie de Fermi anisotrópica donde la invarianza rotacional está rota dando lugar a una fase nemática.

Solo los pasos más relevantes de los cálculos se muestran en la parte central del artículo, los detalles se presentan en los Apéndices.

\subsection{Espinores acoplados en un espacio curvo}

Trabajaremos sobre un geometría genérica de la forma:

$$
d s^{2}=L^{2}\left(-f(z) d t^{2}+g(z) d z^{2}+\frac{d x^{2}+d y^{2}}{z^{2}}\right)
$$

con un campo electromagnético dado por:

$$
A=\frac{Q L}{\kappa} h(z) d t
$$

Aquí $f(z), g(z)$ and $h(z)$ son funciones de la coordenada holográfica $z$. Una elección particular de tales funciónes determinan el fondo que usaremos, el cual es una solución de las ecuaciones de Einstein con una posible contribución de materia al tensor de energía-momento. El borde está localizado a $z=0$ y la geometría se extiende en el IR en la dirección de $z$ crecientes. Las secciones de $z$ constante corresponden a un espacio euclídeo de dos dimensiones. Para pequeños valores de $z$ las funciónes de la métrica son elegidas para que asintóticamente tiendan a la métrica de AdS: $f(z) \rightarrow 1 / z^{2}, g(z) \rightarrow 1 / z^{2}$. El potencial eléctrico tiende a $h(z) \rightarrow z+h_{0}$ donde la constante aditiva $h_{0}$ es determinada en términos de los otros parámetros imponiendo adecuadas condiciones de regularidad en la dimensión extra. Ejemplos específicos de este tipo de geometría son: agujeros negros cargados [109], estrellas de electrones [115]-[118], así como estrellas de electrones a temperatura finita [119]. En este trabajo mantendremos el formalismo lo más general posible pero teniendo en mente que lo aplicaremos al espacio-tiempo de [115].

En la geometría (455) propagamos un campo espinorial de carga $q$, de acuerdo a la acción:

$$
S=\int d^{4} x\left(\bar{\psi}_{\bar{\sigma}}\left(\Gamma^{M} \mathcal{D}_{M}\right)_{\bar{\sigma} \sigma} \psi_{\sigma}+T_{\bar{\sigma} \bar{\sigma}^{\prime} \sigma \sigma^{\prime}} \psi_{\bar{\sigma}}^{\dagger} \psi_{\bar{\sigma}}^{\dagger} \psi_{\sigma} \psi_{\sigma^{\prime}}\right)
$$

donde $\mathcal{D}_{M}$ representa la derivada covariante en espacio-tiempo curvo y contribuciones de calibre, $\Gamma^{M}$ son las matrices de Dirac en un espacio tiempo curvo, con los índices $M=t, x, y, z$, y las $\sigma$ son índices espinoriales, que corren de 1 a 4 y donde una suma siempre será entendida cuando los índices se repitan. El tensor invariante de Lorentz $T_{\bar{\sigma} \bar{\sigma}^{\prime} \sigma \sigma^{\prime}}$ tiene 4 índices espinoriales y estd́ado por:

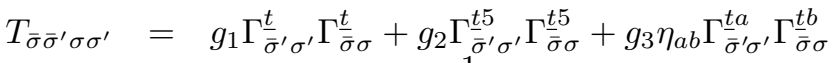

$$
\begin{aligned}
& +g_{4} \eta_{a b} \Gamma_{\bar{\sigma}^{\prime} \sigma^{\prime}}^{t a 5} \Gamma_{\bar{\sigma} \sigma}^{t b 5}-\frac{1}{4} g_{5}\left(\Gamma^{\underline{t}} \Gamma^{[a b]} \Gamma^{5}\right)_{\bar{\sigma}^{\prime} \sigma^{\prime}}\left(\Gamma^{\underline{t}} \Gamma_{[a b]} \Gamma^{5}\right)_{\bar{\sigma} \sigma}
\end{aligned}
$$

Aquí $\Gamma^{a}$ son las matrices de Dirac en el espacio tangente con el índice $a, b=\underline{0}, \underline{1}, \underline{2}, \underline{3}$ donde de nuevo una suma es siempre intentada siempre que el índice se repita, $\Gamma^{a b}=\Gamma^{a} \Gamma^{b}$ and $\Gamma^{[a b]}=\left[\Gamma^{a}, \Gamma^{b}\right]$. Ésta es la interacción cuártica fermiónica covariante más general que puede ser escrita en un espacio curvo [116]. Depende de cinco coeficientes $\left(g_{1}, \ldots, g_{5}\right)$, donde $g_{1}$ controla la interacción densidad-densidad, $g_{2}$ la densidad quiral-densidad quiral, $g_{3}$ el acoplamiento corriente-corriente, $g_{4}$ el corriente quiral-corriente quiral, y $g_{5}$ controla el acoplamiento cuadrático en el tensor de corriente quiral.

En lo que sigue descompondremos el campo espinorial en modos fermiónicos libres y reescribiremos la dinámica en términos de ellos. Resolviendo la dependencia en $z$ en el límite semi-clásico, interpretaremos el sistema de arriba como un sistema multi-fermiónico en un espacio plano de dos dimensiones.

\subsection{Estados fermiónicos libres en la aproximación WKB}

Para encontrar una base para descomponer nuestro campo espinorial necesitamos resolver las perturbaciones fermiónicas libres que satisfacen la ecuación de Dirac

$$
\left(\Gamma^{M} \mathcal{D}_{M}-m\right)_{\sigma \bar{\sigma}} \psi_{\bar{\sigma}}=0
$$


Imponiendo una solución normalizable con condiciones de borde tipo hard-wall ("pared dura", HW) en el IR, después de separar variables, la solución general de esta ecuación puede ser escrita como:

$$
\psi^{\sigma}(t, \vec{x}, z)=\sum_{\alpha n \vec{k}} c_{\alpha n \vec{k}}(t) \psi_{\alpha n \vec{k}}^{\sigma}(z) e^{i \vec{k} \cdot \vec{x}}
$$

en términos de autofunciónes $\psi_{\alpha n \vec{k}}^{\sigma}(z)$, con $\alpha= \pm 1$ un índice de espin, $\vec{k}$ un momento bidimensional en el plano $x y$, y $n$ un índice (genéricamente discreto) que etiqueta las soluciones de la ecuación dependiente de la coordenada $z$. Ya que la ecuación de Dirac es rotacionalmente invariante en el plano $x y$, podemos concentrarnos en las soluciones con un momento que apunte a lo largo del eje $x$ positivo, desde la cual todos las otras soluciones pueden ser obtenidas vía una rotación. En otras palabras, tenemos

$$
\psi_{\alpha n \vec{k}}^{\sigma}(z)=D_{\sigma \bar{\sigma}}(\theta) \psi_{\alpha n k}^{\bar{\sigma}}(z)
$$

donde $D(\theta)$ es una $x y$ matriz de rotación en la representación espinorial, que mapea el eje $x$ en la dirección de $\vec{k}$ que forma un angulo $\theta$ con este. Se escribe como

$$
D_{\sigma \bar{\sigma}}(\theta)=\cos \frac{\theta}{2} \delta_{\sigma \bar{\sigma}}+\sin \frac{\theta}{2} \Gamma_{\sigma \bar{\sigma}}^{x y}
$$

Todas las componentes espinoriales $\psi_{\alpha n k}^{\bar{\sigma}}(z)$ con $\sigma=1 \ldots 4$ pueden ser escritas en términos de una única función $\phi_{k n}(z)$ que satisface una ecuación de Scrhödinger efectiva con algún potencial efectivo complicado. Tal ecuación puede en principio ser resuelta numéricamente como en [118], pero para nuestros fines sera suficiente usar el límite WKB presentado en [117] adaptado a nuestras condiciones de borde de HW (ver Apéndices).

Las frecuencias $\hat{\omega}_{n}(k)$ son elegidas para que satisfagan la condiciones de cuantización de Bohr-Sommerfeld:

$$
\gamma \int_{z_{0}}^{z_{m}} d z \sqrt{g(z)} \sqrt{\frac{1}{f(z)}\left(\hat{\omega}_{n}(k)+h(z)\right)^{2}-z^{2} \hat{k}^{2}-\hat{m}^{2}}=\left(n-\frac{1}{4}\right) \pi
$$

donde $z_{m}$ es la posición del HW y $\gamma=q Q L / \kappa$. Ecuación (463) es válida en el caso de un único punto de retorno como es explicado en [117], para grandes valores de $\gamma$. Si el potencial efectivo de Schrödinger desarrolla tres puntos de retorno las correspondientes fórmulas se vuelven más complicadas.

\subsection{Interacciones de fermiones en el espacio-tiempo holográfico.}

La descomposición (460) nos permite reescribir la dinámica como un hamiltoniano en la segunda cuantificación para múltiples fermiones en un sistema bidimensional en el cual el índice $n$ denota especies de fermión. En efecto, si en la expansión (460) promovemos los coeficientes $c_{\alpha n \vec{k}}$ a operadores e la insertamos en el hamiltoniano obtenido de la acción (457), obtenemos (see Apéndice 8.13):

$$
H / \gamma=\sum_{\vec{k} \alpha n} \hat{\omega}_{n}(k) c_{\alpha n \vec{k}}^{\dagger} c_{\alpha n \vec{k}}+\sum_{\substack{\vec{k} \overrightarrow{k^{\prime}} \vec{q} \\ \bar{\alpha} \bar{n} \bar{\alpha}^{\prime} \bar{n}^{\prime} \alpha n \alpha^{\prime} n^{\prime}}} \hat{T}_{\bar{\alpha} \bar{n} \bar{\alpha}^{\prime} \bar{n}^{\prime} \alpha n \alpha^{\prime} n^{\prime}}^{\left(\vec{k}+\overrightarrow{k^{\prime}}-\vec{k}\right) \vec{k} \vec{k}^{\prime}} c_{\bar{\alpha} \bar{n}(\vec{k}+\vec{q})}^{\dagger} c_{\bar{\alpha}^{\prime} \bar{n}^{\prime}\left(\vec{k}^{\prime}-\vec{q}\right)}^{\dagger} c_{\alpha n \vec{k}} c_{\alpha^{\prime} n^{\prime} \vec{k}^{\prime}}
$$

donde $c_{\alpha n \vec{k}}^{\dagger}$ es el operador de creación de un fermión del tipo $n$ con momento $\vec{k}$ y espín $\alpha$, y $c_{\alpha n \vec{k}}$ es el operador de aniquilación correspondiente. Vemos que las frecuencias $\hat{\omega}_{n}(k)$ juegan el rol de relación de dispersión. Por otro lado el tensor $\hat{T}_{\bar{\alpha}} \vec{k} \bar{k}_{\bar{n}}^{\prime} \vec{k}^{\prime} \vec{n}^{\prime} \alpha n \alpha^{\prime} n^{\prime}$ contiene la información acerca de la fuerza de la interacción, y puede ser obtenida contrayendo el tensor de interacción en espacio de posición $T_{\bar{\sigma} \bar{\sigma}^{\prime} \sigma \sigma^{\prime}}$ con los autoestados de fermiones libres $\psi_{\alpha n \vec{k}}(z)$ (ver Apéndice 8.13). Hacemos notar que hemos re-escaleado por un factor $\gamma$ los acoplamientos: $g_{1} \ldots g_{5} \gamma \rightarrow g_{1} \ldots \gamma g_{5}$.

Dado el hamiltoniano (464), el estado base está caracterizado por un cierto conjunto de números de ocupación $N_{\alpha n \vec{k}}$, y una excitación puede ser considerada como variaciones de tal conjunto: $\delta N_{\alpha n \vec{k}}$. De acuerdo a la teoría de Landau del líquido de Fermi, la energía de una exitación se escribe como

$$
\delta E / \gamma=\int d^{2} k \epsilon_{\alpha n}(\vec{k}) \delta N_{\alpha n \vec{k}}+\frac{1}{2} \int d^{2} k d^{2} k^{\prime} f_{\alpha n \alpha^{\prime} n^{\prime}}\left(\vec{k}, \vec{k}^{\prime}\right) \delta N_{\alpha n \vec{k}} \delta N_{\alpha^{\prime} n^{\prime} \vec{k}^{\prime}}
$$

donde $\epsilon_{\alpha n}(\vec{k})$ es la "relación de dispersión renormalizada" para las cuasi-partículas y $f_{\alpha n \alpha^{\prime} n^{\prime}}\left(\vec{k}, \vec{k}^{\prime}\right)$ es su "función de interacción". Siguiendo [86], estas funciones pueden ser escritas más o menos directamente de 


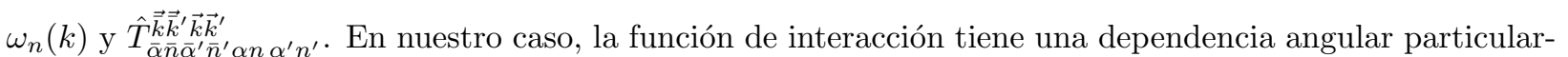
mente simple, que puede ser descompuesta como (ver Apéndice 8.13):

$$
f_{\alpha n \alpha^{\prime} n^{\prime}}\left(\vec{k}, \vec{k}^{\prime}\right)=f_{\alpha n \alpha^{\prime} n^{\prime}}^{1}\left(k, k^{\prime}\right)+f_{\alpha n \alpha^{\prime} n^{\prime}}^{c}\left(k, k^{\prime}\right) \cos \left(\theta^{\prime}-\theta\right)+f_{\alpha n \alpha^{\prime} n^{\prime}}^{s}\left(k, k^{\prime}\right) \sin \left(\theta^{\prime}-\theta\right)
$$

en términos de los llamados "parámetros de Landau" cuya forma explícita es :

$$
\begin{aligned}
& \left.f_{\alpha n \alpha^{\prime} n^{\prime}}^{1}\left(k, k^{\prime}\right)=\sum_{\sigma_{i} \bar{\sigma}_{i}}\left(T_{\sigma_{1} \sigma_{2}\left[\sigma_{3} \sigma_{4}\right]}--T_{\sigma_{1} \bar{\sigma}_{2}\left[\sigma_{3} \bar{\sigma}_{4}\right.} \Gamma_{\bar{\sigma}^{4}}^{\underline{x}} \sigma^{4}\right] \Gamma^{\underline{\underline{x}} \underline{y}} \sigma^{2}\right) \\
& \times \quad \int d z \sqrt{-g} \bar{\psi}_{\alpha n k}^{\sigma^{1}}(z) \bar{\psi}_{\alpha^{\prime} n^{\prime} k^{\prime}}^{\sigma^{2}}(z) \psi_{\alpha^{\prime} n^{\prime} k^{\prime}}^{\sigma^{3}}(z) \psi_{\alpha n k}^{\sigma^{4}}(z) \\
& f_{\alpha n \alpha^{\prime} n^{\prime}}^{c}\left(k, k^{\prime}\right)=\sum_{\sigma_{i} \bar{\sigma}_{i}}\left(T_{\sigma_{1} \sigma_{2}\left[\sigma_{3} \sigma_{4}\right]}+T_{\sigma_{1} \bar{\sigma}_{2}\left[\sigma_{3} \bar{\sigma}_{4}\right.} \Gamma_{\left.\overline{\bar{\sigma}^{4}} \sigma^{4}\right]}^{\underline{x y}} \Gamma^{\underline{\underline{\sigma^{2}}} \sigma^{2}}\right) \\
& \times \quad \int d z \sqrt{-g} \bar{\psi}_{\alpha n k}^{\sigma^{1}}(z) \bar{\psi}_{\alpha^{\prime} n^{\prime} k^{\prime}}^{\sigma^{2}}(z) \psi_{\alpha^{\prime} n^{\prime} k^{\prime}}^{\sigma^{3}}(z) \psi_{\alpha n k}^{\sigma^{4}}(z) \\
& f_{\alpha n \alpha^{\prime} n^{\prime}}^{s}\left(k, k^{\prime}\right)=\sum_{\sigma_{i} \bar{\sigma}_{i}}\left(T_{\sigma_{1} \sigma_{2}\left[\sigma_{3} \bar{\sigma}_{4}\right.} \Gamma_{\left.\bar{\sigma}^{4} \sigma^{4}\right]}^{\underline{x y}}-T_{\sigma_{1} \bar{\sigma}_{2}\left[\sigma_{3} \sigma_{4}\right]} \Gamma^{\underline{x} \underline{\underline{\sigma}}} \sigma^{2}\right) \\
& \times \quad \int d z \sqrt{-g} \bar{\psi}_{\alpha n k}^{\sigma^{1}}(z) \bar{\psi}_{\alpha^{\prime} n^{\prime} k^{\prime}}^{\sigma^{2}}(z) \psi_{\alpha^{\prime} n^{\prime} k^{\prime}}^{\sigma^{3}}(z) \psi_{\alpha n k}^{\sigma^{4}}(z)
\end{aligned}
$$

Por otro lado, la relación de dispersión renormalizada puede ser obtenida dentro de una aproximación de campo medio como:

$$
\begin{aligned}
\epsilon_{\alpha n}(\vec{k}) & =\hat{\omega}_{n}(k)+\sum_{\alpha^{\prime} n^{\prime} \vec{k}^{\prime}} f_{\alpha n \alpha^{\prime} n^{\prime}}\left(\vec{k}, \vec{k}^{\prime}\right) N_{\alpha^{\prime} n^{\prime} \vec{k}^{\prime}} \\
N_{\alpha n \vec{k}} & =\frac{1}{1+e^{-\beta \epsilon_{\alpha n}(\vec{k})}}
\end{aligned}
$$

Estas ecuaciones acopladas deben ser resueltas auto-consistentemente. El parámetro $\beta$ representa la inversa de la temperatura del baño térmico con el cual los fermiones están en equilibrio, que en nuestro caso identificamos con la temperatura del "espacio-tiempo curvo". Ya que estamos trabajando con una solución de temperatura nula, tomaremos el límite $\beta \rightarrow 0$ y la función $N_{\alpha n \vec{k}}$ se convierte $N_{\alpha n \vec{k}}=H\left(-\epsilon_{\alpha n}(\vec{k})\right)$ donde $H(x)$ es la función paso de Heaviside que se anula para $x<0$ y toma el valor 1 para $x>0$. Podemos definir el "momento de Fermi" $k_{F}^{n \alpha}$ de la componente $\alpha$ de espín del modo $n$ como $\epsilon_{\alpha n}\left(k_{F}^{n \alpha}\right)=0$. Esto nos permite reescribir las ecuaciones de auto-consistencia (468)-(469) como condiciones sobre el momento de Fermi:

$$
\omega_{n}\left(k_{F}^{n \alpha}\right)+\sum_{\alpha^{\prime} n^{\prime}} \int_{0}^{k_{F}^{n^{\prime} k^{\prime}}} d k^{\prime} k^{\prime} f_{\alpha n \alpha^{\prime} n^{\prime}}^{1}\left(k_{F}^{n \alpha}, k_{F}^{n^{\prime} \alpha^{\prime}}\right)=0
$$

Notemos que la condición de existencia de $k_{F}^{n^{\prime} k^{\prime}}$ pone un límite superior en el número de modos: $n \leq n_{\max }$, que necesitamos que sea considerado en la suma. Modos tales que $\epsilon_{\alpha n}(k)>0$ para todo $k$, no contribuye a la formula (470). De aquí usando la fórmula de Landau (465), los cálculos usuales de Pomeranchuk se aplican (ver Apéndice 8.15), y entonces podemos ver directamente que el estado del sistema resulta inestable si cualquiera de las siguientes condiciones es violada:

$$
\begin{gathered}
\forall N:\left|k_{F}^{\alpha n}\left(\delta_{\alpha n \alpha^{\prime} n^{\prime}}+2 \pi k_{F}^{\alpha^{\prime} n^{\prime}} f_{\alpha n \alpha^{\prime} n^{\prime}}^{1}\left(k_{F}^{\alpha n}, k_{F}^{\alpha^{\prime} n^{\prime}}\right)\right)\right|_{N \times N}>0 \\
\forall N:\left|k_{F}^{\alpha n}\left(\delta_{\alpha n \alpha^{\prime} n^{\prime}}+\pi k_{F}^{\alpha^{\prime} n^{\prime}} f_{\alpha n \alpha^{\prime} n^{\prime}}^{c}\left(k_{F}^{\alpha n}, k_{F}^{\alpha^{\prime} n^{\prime}}\right)\right)\right|_{N \times N}>0 \\
\forall N: \quad \mid 2 k_{F}^{\alpha n}\left(\delta_{\alpha n \alpha^{\prime} n^{\prime}}+\pi k_{F}^{\alpha^{\prime} n^{\prime}} f_{\alpha n \alpha^{\prime} n^{\prime}}^{c}\left(k_{F}^{\alpha n}, k_{F}^{\alpha^{\prime} n^{\prime}}\right)\right) \\
\quad-\left.\quad \pi k_{F}^{\alpha n} k_{F}^{\alpha^{\prime} n^{\prime}}\left(f_{\alpha n \alpha^{\prime} n^{\prime}}^{s}\left(k_{F}^{\alpha n}, k_{F}^{\alpha^{\prime} n^{\prime}}\right)-f_{\alpha^{\prime} n^{\prime} \alpha n}^{s}\left(k_{F}^{\alpha n}, k_{F}^{\alpha^{\prime} n^{\prime}}\right)\right)\right|_{N \times N}>0
\end{gathered}
$$

donde ||$_{N \times N}$ es el $N$-ésimo menor principal dominante, que es el determinante de la sub-matriz $N \times N$ de la esquina superior izquierda, $N=1, ; n_{\max }$. Estas condiciones son fáciles de implementar recursivamente, y nos permiten construir un diagrama de fase como una función de los parámetros del espacio-tiempo curvo y los acoplamientos. 


\subsection{Apéndice D1: el método de Pomeranchuk}

Ya que los cálculos de Pomeranchuk no son usados extensamente fuera del contexto de la materia condensada, daremos en esta sección una breve introducción a los mismos. Nos limitaremos al caso de dos dimensiones que es el que nos interesa. Explicaremos el método para un único fermión, la generalización para múltiples especies de fermiones es dada en el Apéndice 8.15.

Dado un fermión en dos dimensiones a temperatura cero con una relación de dispersión $\epsilon(k)$ (en la cual incluimos un posible potencial químico), su superficie de Fermi es definida en el espacio de momentos por la relación $\epsilon\left(k_{F}\right)=0$. En el estado fundamental todos los estados con momento $k \leq k_{F}$ están ocupados $n(k)=1$, mientras que una deformación de tal superficie de Fermi tiene una energía a acoplamiento débil dada por la fórmula de Landau:

$$
\delta E=\int d^{2} k \epsilon(k) \delta n(\vec{k})+\frac{1}{2} \int d^{2} k d^{2} k^{\prime} f\left(\vec{k}, \vec{k}^{\prime}\right) \delta n(\vec{k}) \delta n\left(\vec{k}^{\prime}\right)
$$

donde $f\left(\vec{k}, \vec{k}^{\prime}\right)$ es la llamada "función de interacción" que parametríza la interacción entre cuasi-partículas con momento $\vec{k}$ y $\vec{k}^{\prime}$, y la variación $\delta n(\vec{k})=0, \pm 1$ en los números de ocupación del estado con momento $\vec{k}$ puede ser escrita como:

$$
\delta n(\vec{k})=H(-\epsilon(k)+\delta g(\vec{k}))-H(-\epsilon(k))
$$

donde $H(x)$ es la función paso de Heaviside que es 1 para valores positivos de $x$ y 0 para valores negativos de $x$, y $\delta g(\vec{k})$ es una función auxiliar que parametríza la deformación de la superficie de Fermi. Usando la expansión de la distribución de la Heaviside $H$ podemos escribir

$$
\delta n(\vec{k})=\delta(-\epsilon(k)) \delta g(\vec{k})+\frac{1}{2} \delta^{\prime}(-\epsilon(k)) \delta g(\vec{k})^{2}
$$

(este es un boceto de cálculo, si el lector no se siente confortable con el mismo, el cálculo puede ser repetido reemplazando $H(x)$ por la distribución de Fermi $F(x)=1 /(\exp (\beta x)+1)$ y entonces tomar el límite $\beta \rightarrow \infty$.) Cuando reemplazamos esto en (474), las funciones delta de Dirac fuerzan el integrando a ser evaluado en $\epsilon(k)=0$, a saber en la superficie de Fermi. Usando coordenadas polares en el espacio de momentos $(k, \theta)$, esto implica que la integral sobre el momento desaparece y el integrando es evaluado a $k=k_{F}$, quedando solo una dependencia angular:

$$
\delta E=\frac{k_{F}}{2}\left(\int d \theta \delta g(\theta)^{2}+k_{F} \int d \theta d \theta^{\prime} f\left(\theta-\theta^{\prime}\right) \delta g(\theta) \delta g\left(\theta^{\prime}\right)\right)
$$

donde $f\left(\theta-\theta^{\prime}\right)$ es la función de interacción evaluada en la superficie de Fermi $f\left(k_{F}, \theta, k_{F}, \theta^{\prime}\right)$ que debida a la invarianza rotacional, puede solo depender de la diferencia $\theta-\theta^{\prime}$. Usando una base de funciones $\{\cos (m \theta), \sin (m \theta)\}_{m \in \mathbb{N}_{0}}$ podemos hacer la siguiente descomposición:

$$
f\left(\theta-\theta^{\prime}\right)=\sum_{m=0}\left(f_{c}^{m} \cos \left(m\left(\theta-\theta^{\prime}\right)\right)+f_{s}^{m} \sin \left(m\left(\theta-\theta^{\prime}\right)\right)\right)
$$

$\mathrm{y}$

$$
\delta g(\theta)=\sum_{m=0}\left(\delta g_{c}^{m} \cos (m \theta)+\delta g_{s}^{m} \sin (m \theta)\right)
$$

Remplazando esto en la energía de la excitación obtenemos:

$$
\delta E=\pi k_{F} \delta g_{c}^{0^{2}}\left(1+2 \pi k_{F} f_{c}^{0}\right)+\frac{\pi k_{F}}{2} \sum_{m=1}\left(\delta g_{c}^{m 2}+\delta g_{s}^{m 2}\right)\left(1+\pi k_{F} f_{c}^{m}\right)
$$

Vemos que la energía de una excitación termina escribiéndose como una forma cuadrática en los parámetros de la deformación. Para tener un sistema estable, $\delta E$ tiene que ser una cantidad positiva para cualquier posible excitación, o en otras palabras para cualquier valor posible de $\delta g(\theta)$. Esto implica que la forma cuadrática definida arriba debe ser definida positiva, de donde obtenemos las condiciones de estabilidad:

$$
1+2 \pi k_{F} f_{c}^{0}>0 \quad ; \quad \forall m>0: 1+\pi k_{F} f_{c}^{m}>0
$$

Si alguna de estas condiciones son violadas el sistema se vuelve inestable. Notemos que los parámetros $f_{s}^{m}$ desaparecen completamente de los cálculos. Los parámetros $f_{c}^{m}$ son llamados los "parámetros de Landau " del líquido de Fermi.

El procedimiento de arriba puede ser fácilmente generalizado a muchas especies de fermiones, el resultado es también una forma cuadrática no diagonal en las componentes de espín y en los índices de especies. Los detalles son dados en el Apéndice (8.15) 


\subsection{Apéndice D2: estados fermiónicos libres en el espacio-tiempo curvo holográfico}

La métrica del espacio-tiempo tiene la siguiente forma general:

$$
d s^{2}=L^{2}\left(-f(z) d t^{2}+g(z) d z^{2}+\frac{d x^{2}+d y^{2}}{z^{2}}\right)
$$

con campos de calibres dados por

$$
A_{t}=\frac{Q L}{\kappa} h(z) \quad A_{i}=0
$$

La métrica puede ser generado con los vierbein:

$$
e_{M}^{a}=\left\{\begin{array}{l}
e_{t}^{\frac{t}{t}}=L \sqrt{f(z)} \\
e^{\frac{z}{z}}=L \sqrt{g(z)} \\
e_{i}^{\frac{j}{z}}=\frac{L}{z} \delta_{i}^{\frac{j}{j}}
\end{array}\right.
$$

como:

$$
e_{M}^{a} e_{N}^{b} \eta_{a b}=g_{M N}
$$

Aquí los índices latinos en minúscula $a, b=\underline{0}, \underline{1}, \underline{2}, \underline{3}$ indican las direcciones del espacio tangente, cuando ellos aparezcan explícitamente subrayados, y los índices latinos con mayúscula $A, B=0,1,2,3$ indican las direcciones del espacio-tiempo. Los índices espaciales están indicados como $i, j=1,2$ (respectivamente $\underline{i}, \underline{j}=\underline{1}, \underline{2})$. Los inversos de los vierbein están dados por:

$$
e_{a}^{M}=\left\{\begin{array}{l}
e_{\underline{t}}^{t}=\frac{1}{L \sqrt{f(z)}} \\
e_{\underline{z}}^{z}=\frac{1}{L \sqrt{g(z)}} \\
e_{\underline{j}}^{i}=\frac{z}{L} \delta_{\underline{j}}^{i}
\end{array}\right.
$$

Los símbolos de Christoffel resultantes se escriben:

$$
\begin{aligned}
\Gamma_{R M}^{N} & =\frac{1}{2} g^{N S}\left(g_{R S, M}+g_{M S, R}-g_{R M, S}\right) \\
& =\frac{1}{2} g^{N S}\left(g_{R S, z} \delta_{M z}+g_{M S, z} \delta_{R z}\right)-\frac{1}{2} g_{R M, z} g^{N z}
\end{aligned}
$$

o más explícitamente:

$$
\begin{aligned}
\Gamma_{R t}^{N} & =\frac{1}{2} g^{t t} g_{t t, z} \delta^{N t} \delta_{R z}-\frac{1}{2} g^{z z} g_{t t, z} \delta^{N z} \delta_{R t} \\
& =\frac{f^{\prime}(z)}{2 f(z)} \delta^{N t} \delta_{R z}-\frac{f^{\prime}(z)}{2 g(z)} \delta^{N z} \delta_{R t} \\
\Gamma_{R i}^{N} & =\frac{1}{2} g^{i i} g_{i i, z} \delta^{N i} \delta_{R z}-\frac{1}{2} g^{z z} g_{i i, z} \delta^{N z} \delta_{R i} \\
& =\frac{1}{z^{3} g(z)} \delta^{N z} \delta_{R i}-\frac{1}{z} \delta^{N i} \delta_{R z} \\
\Gamma_{R z}^{N} & =\frac{1}{2} g^{t t} g_{t t, z} \delta^{N t} \delta_{R t}+\frac{1}{2} g^{z z} g_{z z, z} \delta^{N z} \delta_{R z}+\frac{1}{2} g^{i i} g_{i i, z} \delta^{N i} \delta_{R i} \\
& =\frac{f^{\prime}(z)}{2 f(z)} \delta^{N t} \delta_{R t}+\frac{g^{\prime}(z)}{2 g(z)} \delta^{N z} \delta_{R z}-\frac{1}{z} \delta^{N i} \delta_{R i}
\end{aligned}
$$

Concluimos que:

$$
\begin{aligned}
\Gamma_{t t}^{z} & =-\frac{f^{\prime}(z)}{2 g(z)} \quad \Gamma_{i i}^{z}=\frac{1}{z^{3} g(z)} \quad \Gamma_{z}^{z}=\frac{g^{\prime}(z)}{2 g(z)} \\
\Gamma_{z t}^{t} & =\Gamma_{t z}^{t}=\frac{f^{\prime}(z)}{2 f(z)} \\
\Gamma_{z i}^{i} & =\Gamma_{i z}^{i}=-\frac{1}{z}
\end{aligned}
$$


La conexión de espín puede escribirse como:

$$
\omega_{M}^{a b}=e_{N}^{a} \partial_{M} e^{N b}+e_{N}^{a} e^{R b} \Gamma_{R M}^{N}
$$

Más explícitamente:

$$
\begin{aligned}
\omega_{t}^{a b} & =e_{t}^{a} e^{z b} \Gamma_{z t}^{t}+e_{z}^{a} e^{t b} \Gamma_{t t}^{z} \\
& =\left(\delta^{a \underline{t}} \delta^{b \underline{z}}-\delta^{a \underline{z}} \delta^{b \underline{t}}\right) \frac{f^{\prime}(z)}{2 \sqrt{f(z) g(z)}} \\
\omega_{i}^{a b} & =e_{i}^{a} e^{z b} \Gamma_{z i}^{i}+e_{z}^{a} e^{i b} \Gamma_{i i}^{z} \\
& =\left(\delta^{a \underline{z}} \delta^{b \underline{i}}-\delta^{a} \underline{i} \delta^{b \underline{z}}\right) \frac{1}{z^{2} \sqrt{g(z)}} \\
\omega_{z}^{a b} & =0
\end{aligned}
$$

Concluyendo que las componentes no nulas son:

$$
\omega_{t}^{\underline{t z}}=-\omega_{t}^{\underline{z t}}=\frac{f^{\prime}(z)}{2 \sqrt{f(z) g(z)}} \quad \omega_{i}^{\underline{z i}}=-\omega_{i}^{i z}=\frac{1}{z^{2} \sqrt{g(z)}} \quad \omega_{z}^{a b}=0
$$

\subsection{Apéndice D3: la ecuación de Dirac}

La ecuación de Dirac en un espacio curvo se escribe:

$$
\left(\Gamma^{M} \mathcal{D}_{M}-m\right) \psi=0
$$

donde la derivada covariante está dada por:

$$
\mathcal{D}_{M}=\partial_{M}+\frac{1}{4} \omega_{a b M} \Gamma^{a b}-i q A_{M}
$$

Aquí $\Gamma^{a b}=\frac{1}{2}\left[\Gamma^{a} ; \Gamma^{b}\right]$ donde $\Gamma^{a}$ son las matrices gamma en espacio plano. Escribiendo parte por parte:

$$
\begin{aligned}
\Gamma^{z} \mathcal{D}_{z} & =e_{a}^{z} \Gamma^{a} \partial_{z} \\
& =\frac{1}{L \sqrt{g(z)}} \Gamma^{\underline{z}} \partial_{z} \\
\Gamma^{i} \mathcal{D}_{i} & =e_{a}^{i} \Gamma^{a}\left(\partial_{i}+\frac{1}{4} \omega_{a b i} \Gamma^{a b}\right) \\
& =\frac{z}{L} \Gamma^{\underline{i}} \partial_{i}-\frac{1}{z L \sqrt{g(z)}} \Gamma^{\underline{z}} \\
\Gamma^{t} \mathcal{D}_{t} & =e_{a}^{t} \Gamma^{a}\left(\partial_{t}+\frac{1}{4} \omega_{a b t} \Gamma^{a b}-i q A_{t}\right) \\
& =\frac{1}{L \sqrt{f(z)}} \Gamma^{\underline{t}}\left(\partial_{t}-i \frac{q Q L}{\kappa} h(z)\right)+\frac{f^{\prime}(z)}{4 L f(z) \sqrt{g(z)}} \Gamma^{\underline{z}}
\end{aligned}
$$

Usando lo de arriba obtenemos:

$$
\left(\frac{1}{\sqrt{g(z)}} \Gamma^{\underline{z}}\left(\partial_{z}-\frac{1}{z}+\frac{f^{\prime}(z)}{4 f(z)}\right)+\frac{1}{\sqrt{f(z)}} \Gamma^{\underline{t}}\left(\partial_{t}-i \frac{q Q L}{\kappa} h(z)\right)+z \Gamma^{\underline{i}} \partial_{i}-m L\right) \psi=0
$$

Esta es la ecuación de Dirac que tenemos que resolver para nuestro espinor $\psi$.

Ahora si re-escaleamos:

$$
\psi=\frac{z f^{-\frac{1}{4}}(z)}{L^{2}} \tilde{\psi}
$$

obtenemos:

$$
\left(\frac{1}{\sqrt{g(z)}} \Gamma^{\underline{z}} \partial_{z}+\frac{1}{\sqrt{f(z)}} \Gamma^{\underline{t}}\left(\partial_{t}-i \frac{q Q L}{\kappa} h(z)\right)+z \Gamma^{\underline{i}} \partial_{i}-m L\right) \tilde{\psi}=0
$$

Separando variables como:

$$
\tilde{\psi}\left(t, x^{i}, z\right)=e^{i\left(k_{i} x^{i}-\omega t\right)} \psi_{\vec{k} \omega}(z)
$$


tenemos:

$$
\left(\frac{1}{\sqrt{g(z)}} \Gamma^{\underline{z}} \partial_{z}-\frac{i}{\sqrt{f(z)}} \Gamma^{\underline{t}}\left(\omega+\frac{q Q L}{\kappa} h(z)\right)+i z \Gamma^{\underline{i}} k_{i}-m L\right) \psi_{\vec{k} \omega}(z)=0
$$

Podemos usar la invarianza rotacional en el plano $x y$ para escribir $R_{i j} k_{j}=\delta_{x}^{i} k$ donde $\vec{k}^{t}=(k \cos \theta, k \sin \theta)$, $\mathrm{y}$ :

$$
R=\left(\begin{array}{cc}
\cos \theta & \sin \theta \\
-\sin \theta & \cos \theta
\end{array}\right)
$$

En términos de los espinores tenemos que:

$$
\psi_{\vec{k} \omega}(z)=D[\theta] \psi_{k \omega}(z)
$$

donde $D[\theta]$ es la matriz de rotación en la representación espinorial:

$$
D[\theta]=e^{-\frac{\theta}{2} \Gamma \underline{x y}}
$$

Además: $\Gamma^{\underline{x y}}=\Gamma^{\underline{x}} \Gamma^{\underline{y}}$ satisface $\left(\Gamma^{\underline{x}} \underline{y}\right)^{2}=-\left(\Gamma^{\underline{x}}\right)^{2}\left(\Gamma^{\underline{y}}\right)^{2}=-1$, lo cual implica:

$$
D[\theta]=\cos \frac{\theta}{2}-\Gamma \frac{x y}{\sin } \frac{\theta}{2}
$$

La ecuación (500) entonces se escribe:

$$
\left(\frac{1}{\sqrt{g(z)}} \Gamma^{\underline{z}} \partial_{z}-\frac{i}{\sqrt{f(z)}} \Gamma^{\underline{t}}\left(\omega+\frac{q Q L}{\kappa} h(z)\right)+i z k \Gamma^{\underline{x}}-m L\right) \psi_{k \omega}(z)=0
$$

Definiendo los proyectores:

$$
\Pi_{\alpha}=\frac{1}{2}\left(1+(-1)^{\alpha} \Gamma^{\underline{z}} \Gamma^{\underline{t}} \Gamma^{\underline{x}}\right)
$$

con $\alpha=1,2$, y los campos proyectados

$$
\psi_{\alpha k \omega}(z)=\Pi_{\alpha} \psi_{k \omega}(z)
$$

obtenemos:

$$
\left(\frac{1}{\sqrt{g(z)}} \Gamma^{\underline{z}} \partial_{z}-\frac{i}{\sqrt{f(z)}} \Gamma^{\underline{t}}\left(\omega+\frac{q Q L}{\kappa} h(z)\right)+i z k \Gamma^{\underline{x}}-m L\right) \psi_{\alpha k \omega}(z)=0
$$

Tomando la siguiente definición de las matrices gamma:

$$
\begin{aligned}
\Gamma^{\underline{z}}=\left(\begin{array}{cc}
\sigma_{3} & 0 \\
0 & \sigma_{3}
\end{array}\right) & \Gamma^{\underline{t}}=\left(\begin{array}{cc}
i \sigma_{1} & 0 \\
0 & i \sigma_{1}
\end{array}\right) \\
\Gamma^{\underline{x}}=\left(\begin{array}{cc}
-\sigma_{2} & 0 \\
0 & \sigma_{2}
\end{array}\right) & \Gamma^{\underline{y}}=\left(\begin{array}{cc}
0 & \sigma_{2} \\
\sigma_{2} & 0
\end{array}\right)
\end{aligned}
$$

los proyectores se escriben:

$$
\Pi_{\alpha}=\frac{1}{2}\left(1+(-1)^{\alpha}\left(\begin{array}{cc}
-1_{2 \times 2} & 0 \\
0 & 1_{2 \times 2}
\end{array}\right)\right)=\left\{\begin{array}{c}
\left(\begin{array}{cc}
1_{2 \times 2} & 0 \\
0 & 0
\end{array}\right) \\
\left(\begin{array}{cc}
0 & 0 \\
0 & 1_{2 \times 2}
\end{array}\right)
\end{array}\right.
$$

y por lo tanto:

$$
\psi_{1 k \omega}(z)=\left(\begin{array}{c}
\Phi_{k \omega}^{1}(z) \\
0
\end{array}\right) \quad \psi_{2 k \omega}(z)=\left(\begin{array}{c}
0 \\
\Phi_{k \omega}^{2}(z)
\end{array}\right)
$$

Esto nos permite escribir:

$$
\left(\frac{\sigma_{3}}{\sqrt{g(z)}} \partial_{z}+\frac{\sigma_{1}}{\sqrt{f(z)}}\left(\omega+\frac{q Q L}{\kappa} h(z)\right)+i z k(-1)^{\alpha} \sigma_{2}-m L\right) \Phi_{k \omega}^{\alpha}(z)=0
$$


Siendo una ecuación de primer orden con condiciones de contorno definidas (ver Apéndice 8.9), de (513) se sigue que:

$$
\Phi_{k \omega}^{2}(z) \propto \Phi_{-k \omega}^{1}(z)
$$

y por lo tanto podemos concentrarnos en lo que sigue en solo una de las proyecciones, por ejemplo $\alpha=2$. Definiendo:

$$
\Phi_{k \omega}^{2}(z)=\left(\begin{array}{c}
\phi_{k \omega}^{1}(z) \\
\phi_{k \omega}^{2}(z)
\end{array}\right)
$$

obtenemos:

$$
\begin{aligned}
& \left(\frac{1}{\sqrt{g(z)}} \partial_{z}-m L\right) \phi_{k \omega}^{1}(z)+\left(\frac{1}{\sqrt{f(z)}}\left(\omega+\frac{q Q L}{\kappa} h(z)\right)+z k\right) \phi_{k \omega}^{2}(z)=0 \\
& \left(-\frac{1}{\sqrt{g(z)}} \partial_{z}-m L\right) \phi_{k \omega}^{2}(z)+\left(\frac{1}{\sqrt{f(z)}}\left(\omega+\frac{q Q L}{\kappa} h(z)\right)-z k\right) \phi_{k \omega}^{1}(z)=0
\end{aligned}
$$

\subsection{Apéndice D4: condiciones de borde}

Cerca del borde, $f(z) \sim g(z) \sim 1 / z^{2}$ y $h$ va a una constante $h_{0}$, y las ecuaciones toman la forma:

$$
\begin{aligned}
& \left(z \partial_{z}-m L\right) \phi_{k \omega}^{1}(z)+z\left(\omega+\frac{q Q L}{\kappa} h_{o}+k\right) \phi_{k \omega}^{2}(z)=0 \\
& \left(z \partial_{z}+m L\right) \phi_{k \omega}^{2}(z)-z\left(\omega+\frac{q Q L}{\kappa} h_{0}-k\right) \phi_{k \omega}^{1}(z)=0
\end{aligned}
$$

Escribiendo:

$$
\begin{aligned}
& \phi_{k \omega}^{1}(z)=A z^{\alpha} \\
& \phi_{k \omega}^{2}(z)=B z^{\beta}
\end{aligned}
$$

obtenemos:

$$
\begin{aligned}
& (\alpha-m L) A z^{\alpha}+\left(\omega+\frac{q Q L}{\kappa} h_{0}+k\right) B z^{\beta+1}=0 \\
& (\beta+m L) B z^{\beta}-\left(\omega+\frac{q Q L}{\kappa} h_{o}-k\right) A z^{\alpha+1}=0
\end{aligned}
$$

En orden para obtener valores independientes de $A$ y $B$ necesitamos asumir que el determinante se anula:

$$
\begin{aligned}
& (\alpha-m L)(\beta+m L) z^{\alpha+\beta}+\left(\left(\omega+\frac{q^{2} L}{\kappa} h_{o}\right)^{2}-k^{2}\right) z^{\alpha+\beta+2} \rightarrow \\
& \rightarrow(\alpha-m L)(\beta+m L) z^{\alpha+\beta}=0
\end{aligned}
$$

de donde obtenemos $\alpha=m L$ y $\beta=-m L$. Imponemos que la solución no normalizable se anule en el borde $(\mathrm{UV})$ :

$$
\phi_{k \omega}^{2}(0)=0
$$

mientras que en el IR imponemos como condición de borde un HW en $z_{m}$ :

$$
\phi_{k \omega}^{1}\left(z_{m}\right)=0
$$




\subsection{Apéndice D5: ecuación de Schrödinger efectiva}

Llamando $\gamma=q Q L / \kappa$ y re-escaleando $\omega=\gamma \hat{\omega}, k=\gamma \hat{k}$ y $m L=\gamma \hat{m}$ tenemos que:

$$
\begin{gathered}
\left(\frac{1}{\sqrt{g(z)}} \partial_{z}-\gamma \hat{m}\right) \phi_{k \omega}^{1}(z)+\gamma\left(\frac{1}{\sqrt{f(z)}}(\hat{\omega}+h(z))+z \hat{k}\right) \phi_{k \omega}^{2}(z)=0 \\
\left(-\frac{1}{\sqrt{g(z)}} \partial_{z}-\gamma \hat{m}\right) \phi_{k \omega}^{2}(z)+\gamma\left(\frac{1}{\sqrt{f(z)}}(\hat{\omega}+h(z))-z \hat{k}\right) \phi_{k \omega}^{1}(z)=0
\end{gathered}
$$

Resolviendo para $\phi_{k \omega}^{2}$ :

$$
\phi_{k \omega}^{2}(z)=\frac{\gamma \hat{m} \phi_{k \omega}^{1}(z)-\frac{1}{\sqrt{g(z)}} \partial_{z} \phi_{k \omega}^{1}(z)}{\gamma\left(\frac{1}{\sqrt{f(z)}}(\hat{\omega}+h(z))+z \hat{k}\right)}
$$

y reemplazando:

$$
-\frac{1}{\gamma \sqrt{g(z)}} \partial_{z}\left(\frac{\frac{1}{\sqrt{g(z)}} \partial_{z} \phi_{k \omega}^{1}(z)}{\frac{1}{\sqrt{f(z)}}(\hat{\omega}+h(z))+z \hat{k}}\right)+\left(\gamma \frac{\hat{m}^{2}-\frac{1}{f(z)}(\hat{\omega}+h(z))^{2}+z^{2} \hat{k}^{2}}{\frac{1}{\sqrt{f(z)}}(\hat{\omega}+h(z))+z \hat{k}}+\frac{1}{\sqrt{g(z)}} \partial_{z}\left(\frac{\hat{m}}{\frac{1}{\sqrt{f(z)}}(\hat{\omega}+h(z))+z \hat{k}}\right)\right) \phi_{k \omega}^{1}(
$$

Definiendo las coordenadas como:

$$
d u=\left(\frac{1}{\sqrt{f(z)}}(\hat{\omega}+h(z))+z \hat{k}\right) \sqrt{g(z)} d z
$$

tenemos:

$$
-\frac{1}{\gamma} \partial_{u}^{2} \phi_{k \omega}^{1}(z(u))+\underbrace{\left(\partial_{u}\left(\frac{\hat{m}}{\frac{1}{\sqrt{f(z)}}(\hat{\omega}+h(z))+z \hat{k}}\right)+\gamma \frac{\hat{m}^{2}-\frac{1}{f(z)}(\hat{\omega}+h(z))^{2}+z^{2} \hat{k}^{2}}{\left(\frac{1}{\sqrt{f(z)}}(\hat{\omega}+h(z))+z \hat{k}\right)^{2}}\right)}_{V(u)=V_{1}(u)+\gamma V_{0}(u)} \phi_{k \omega}^{1}(z(u))=0
$$

o sea, la ecuación de Shrödinger efectiva:

$$
\left(-\frac{1}{\gamma} \partial_{u}^{2}+V(u)\right) \phi_{k \omega}^{1}(z(u))=0
$$

Es fácil chequear que para $z \rightarrow 0$ tenemos $u \sim\left(\hat{\omega}+h_{0}+\hat{k}\right) z$. Por otro lado para $z \rightarrow \infty$ tenemos $u \rightarrow \hat{\omega} z^{z+2} / \sqrt{g_{\infty}}(z+1) \rightarrow \infty$. Entonces nuestras condiciones de borde se escriben:

$$
\begin{aligned}
\phi_{k \omega}^{1}(z(0)) & =0=\phi_{k \omega}^{2}(z(0)) \\
\phi_{k \omega}^{1}\left(z\left(u_{m}\right)\right) & =0
\end{aligned}
$$

donde $u_{m}$ es la localización de nuestro HW en la variable $u$. En términos generales, esta condición de borde implica una condición de cuantización de la forma $\hat{\omega}=\hat{\omega}_{n}(\hat{k}) \operatorname{con} n$ un índice discreto. Entonces el índice $\hat{\omega}$ en nuestras soluciones será reemplazado por el índice $n$.

\subsection{Apéndice D6: forma general de la solución}

Haciendo uso de (530) podemos escribir:

$$
\Phi_{k \omega}^{2}(z)=\left(\begin{array}{c}
\phi_{k \omega}^{1}(z) \\
\frac{\gamma \hat{m} \phi_{k \omega}^{1}(z)-\frac{1}{\sqrt{g(z)}} \partial_{z} \phi_{k \omega}^{1}(z)}{\gamma\left(\frac{1}{\sqrt{f(z)}}(\hat{\omega}+h(z))+z \hat{k}\right)}
\end{array}\right)
$$


y entonces por (514) y (512) tenemos:

$$
\psi_{\alpha k \omega}(z)=\left(\begin{array}{c}
\frac{1-(-1)^{\alpha}}{2} \phi_{-k \omega}^{1}(z) \\
\frac{1-(-1)^{\alpha}}{2} \frac{\gamma \hat{m} \phi_{-k \omega}^{1}(z)-\frac{1}{\sqrt{g(z)}} \partial_{z} \phi_{-k \omega}^{1}(z)}{\gamma\left(\frac{1}{\sqrt{f(z)}}(\hat{\omega}+h(z))-z \hat{k}\right)} \\
\frac{1+(-1)^{\alpha}}{2} \phi_{k \omega}^{1}(z) \\
\frac{1+(-1)^{\alpha}}{2} \frac{\gamma \hat{m} \phi_{k \omega}^{1}(z)-\frac{1}{\sqrt{g(z)}} \partial_{z} \phi_{k \omega}^{1}(z)}{\gamma\left(\frac{1}{\sqrt{f(z)}}(\hat{\omega}+h(z))+z \hat{k}\right)}
\end{array}\right)
$$

Ahora usando (502) obtenemos:

$$
\psi_{\alpha k_{i} \omega}(z)=\left(\begin{array}{cc}
\cos \frac{\theta}{2} 1_{2 \times 2} & \sin \frac{\theta}{2} 1_{2 \times 2} \\
-\sin \frac{\theta}{2} 1_{2 \times 2} & \cos \frac{\theta}{2} 1_{2 \times 2}
\end{array}\right)\left(\begin{array}{c}
\frac{1-(-1)^{\alpha}}{2} \phi_{-k \omega}^{1}(z) \\
\frac{1-(-1)^{\alpha}}{2} \frac{\gamma \hat{m} \phi_{-k \omega}^{1}(z)-\frac{1}{\sqrt{g(z)}} \partial_{z} \phi_{-k \omega}^{1}(z)}{\gamma\left(\frac{1}{\sqrt{f(z)}}(\hat{\omega}+h(z))-z \hat{k}\right)} \\
\frac{1+(-1)^{\alpha}}{2} \phi_{k \omega}^{1}(z) \\
\frac{1+(-1)^{\alpha}}{2} \frac{\gamma \hat{m} \phi_{k \omega}^{1}(z)-\frac{1}{\sqrt{g(z)}} \partial_{z} \phi_{k \omega}^{1}(z)}{\gamma\left(\frac{1}{\sqrt{f(z)}}(\hat{\omega}+h(z))+z \hat{k}\right)}
\end{array}\right)
$$

Teniendo en cuenta la cuantización de la frecuencia, la solución general de la ecuación de Dirac puede ser escrita como:

$$
\psi(x, z, t)=\sum_{\alpha n k_{i}} c_{\alpha n k_{i}}(t) \psi_{\alpha n k_{i}}(z) e^{i \vec{k} \cdot \vec{x}}
$$

con:

$$
\psi_{\alpha n k_{i}}(z)=\left(\begin{array}{cc}
\cos \frac{\theta}{2} 1_{2 \times 2} & \sin \frac{\theta}{2} 1_{2 \times 2} \\
-\sin \frac{\theta}{2} 1_{2 \times 2} & \cos \frac{\theta}{2} 1_{2 \times 2}
\end{array}\right)\left(\begin{array}{c}
\frac{1-(-1)^{\alpha}}{2} \phi_{-k n}^{1}(z) \\
\frac{1-(-1)^{\alpha}}{2} \frac{\gamma \hat{m} \phi_{-k n}^{1}(z)-\frac{1}{\sqrt{g(z)}} \partial_{z} \phi_{-k n}^{1}(z)}{\gamma\left(\frac{1}{\sqrt{f(z)}}\left(\hat{\omega}_{n}(k)+h(z)\right)-z \hat{k}\right)} \\
\frac{1+(-1)^{\alpha}}{2} \phi_{k n}^{1}(z) \\
\frac{1+(-1)^{\alpha}}{2} \frac{\gamma \hat{m} \phi_{k n}^{1}(z)-\frac{1}{\sqrt{g(z)}} \partial_{z} \phi_{k n}^{1}(z)}{\gamma\left(\frac{1}{\sqrt{f(z)}}\left(\hat{\omega}_{n}(k)+h(z)\right)+z \hat{k}\right)}
\end{array}\right)
$$

donde $\phi_{k n}^{1}(z)=\phi_{k \omega_{n}(k)}^{1}(z)$ con $\omega_{n}(k)$ cuantizado de acuerdo a las condiciones de borde.

\subsection{Apéndice D7: solución WKB}

Escribiendo la ecuación de Shrödinger efectiva como:

$$
\left(-\frac{1}{\gamma} \partial_{u}^{2}+V_{1}(u)+\gamma V_{0}(u)\right) \phi_{k \omega}^{1}(z(u))=0
$$

podemos definir:

$$
\phi_{k \omega}^{1}(z(u))=e^{\gamma S(u)}
$$

obteniendo:

$$
-\partial_{u}^{2} S(u)-\gamma\left(\partial_{u} S(u)\right)^{2}+V_{1}(u)+\gamma V_{0}(u)=0
$$

Escribiendo:

$$
S(u)=S_{0}(u)+\frac{1}{\gamma} S_{1}(u)
$$


obtenemos:

$$
-\partial_{u}^{2} S_{0}(u)-\frac{1}{\gamma} \partial_{u}^{2} S_{1}(u)-\gamma\left(\partial_{u} S_{0}(u)+\frac{1}{\gamma} \partial_{u} S_{1}(u)\right)^{2}+V_{1}(u)+\gamma V_{0}(u)=0
$$

Reordenando potencias de $\gamma$ tenemos:

$$
\gamma\left(V_{0}(u)-\partial_{u} S_{0}(u)^{2}\right)-\partial_{u}^{2} S_{0}(u)+V_{1}(u)-2 \partial_{u} S_{0}(u) \partial_{u} S_{1}(u)-\frac{1}{\gamma}\left(\partial_{u}^{2} S_{1}(u)+\partial_{u} S_{1}(u)^{2}\right)=0
$$

En el límite $\gamma \gg 1$ :

$$
\gamma\left(V_{0}(u)-\partial_{u} S_{0}(u)^{2}\right)-\partial_{u}^{2} S_{0}(u)+V_{1}(u)-2 \partial_{u} S_{0}(u) \partial_{u} S_{1}(u)=0
$$

que implica:

$$
\begin{gathered}
V_{0}(u)-\partial_{u} S_{0}(u)^{2}=0 \\
-\partial_{u}^{2} S_{0}(u)+V_{1}(u)-2 \partial_{u} S_{0}(u) \partial_{u} S_{1}(u)=0
\end{gathered}
$$

La primera de estas ecuaciónes puede ser resuelta como:

$$
S_{0}(u)= \pm \int_{u_{o}}^{u} d u \sqrt{V_{0}(u)}
$$

con $u_{o}$ aún indeterminada. Reemplazando en la segunda tenemos que:

$$
\pm \partial_{u} \sqrt{V_{0}(u)}-V_{1}(u) \pm 2 \sqrt{V_{0}(u)} \partial_{u} S_{1}(u)=0
$$

que implica:

$$
S_{1}(u)=-\log V_{0}^{\frac{1}{4}}(u) \pm \int_{u_{o}}^{u} d u \frac{V_{1}(u)}{2 \sqrt{V_{0}(u)}}+c
$$

donde $c$ es una constante. Entonces:

$$
\phi_{k \omega}^{1}=\frac{A_{+}}{V_{0}^{\frac{1}{4}}(u)} e^{\int_{u_{o}}^{u} d u\left(\gamma \sqrt{V_{0}(u)}+\frac{V_{1}(u)}{2 \sqrt{V_{0}(u)}}\right)}+\frac{A_{-}}{V_{0}^{\frac{1}{4}}(u)} e^{-\int_{u_{o}}^{u} d u\left(\gamma \sqrt{V_{0}(u)}+\frac{V_{1}(u)}{2 \sqrt{V_{0}(u)}}\right)}
$$

Notemos que ya que estamos tomando el límite $\gamma \gg 1$, el segundo término en los integrandos pueden ser omitidos:

$$
\phi_{k \omega}^{1}=\frac{A_{+}}{V_{0}^{\frac{1}{4}}(u)} e^{\gamma \int_{u_{o}}^{u} d u \sqrt{V_{0}(u)}}+\frac{A_{-}}{V_{0}^{\frac{1}{4}}(u)} e^{-\gamma \int_{u_{o}}^{u} d u \sqrt{V_{0}(u)}}
$$

Mantendremos esta expresión como está para $V_{0}(u)>0$, pero en la región $V_{0}(u)<0$ escribimos:

$$
\phi_{k \omega}^{1}=\frac{A_{+}}{V_{0}^{\frac{1}{4}}(u)} e^{i \gamma \int_{u_{o}}^{u} d u \sqrt{\left|V_{0}(u)\right|}}+\frac{A_{-}}{V_{0}^{\frac{1}{4}}(u)} e^{-i \gamma \int_{u_{o}}^{u} d u \sqrt{\left|V_{0}(u)\right|}}
$$

La solución de arriba tiene una divergencia obvia a $V_{0}(u)=0$. Identificando $u_{o}$ con un punto de retorno, podemos expandir la ecuación de Schrödinger en su vecindad como:

$$
\left.-\frac{1}{\gamma} \partial_{u}^{2} \phi_{k \omega}(z(u))+\left(V_{1}\left(u_{o}\right)+\left(V_{1}^{\prime}\left(u_{o}\right)+\gamma V_{0}^{\prime}\left(u_{o}\right)\right)\left(u-u_{o}\right)\right)\right) \phi_{k \omega}(z(u))
$$

En el límite $\gamma \gg 1$ obtenemos:

$$
-\frac{1}{\gamma} \partial_{u}^{2} \phi_{k \omega}(z(u))+\gamma V_{0}^{\prime}\left(u_{o}\right)\left(u-u_{o}\right) \phi_{k \omega}(z(u))
$$

Una base de soluciones son las funciones de Airy:

$$
\phi_{k \omega}(z(u))=A_{A i} A i\left(\gamma^{\frac{2}{3}} V_{0}^{\prime \frac{1}{3}}\left(u_{o}\right)\left(u-u_{o}\right)\right)+A_{B i} B i\left(\gamma^{\frac{2}{3}} V_{0}^{\prime \frac{1}{3}}\left(u_{o}\right)\left(u-u_{o}\right)\right)
$$


Resumiendo tenemos:

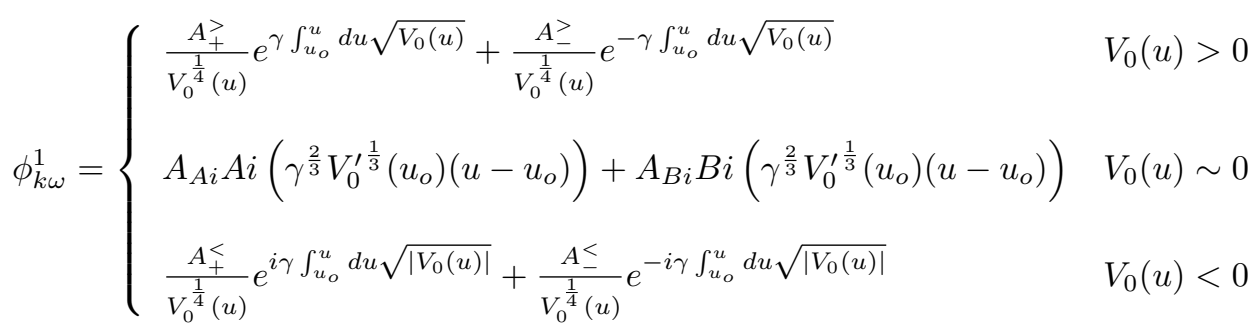

(esto es para el caso de un único punto de retorno, cuando mas puntos de retorno están presentes, necesitamos un análisis mayor).

Usando las expansiones asintóticas de las funciónes de Airy, podemos decir que:

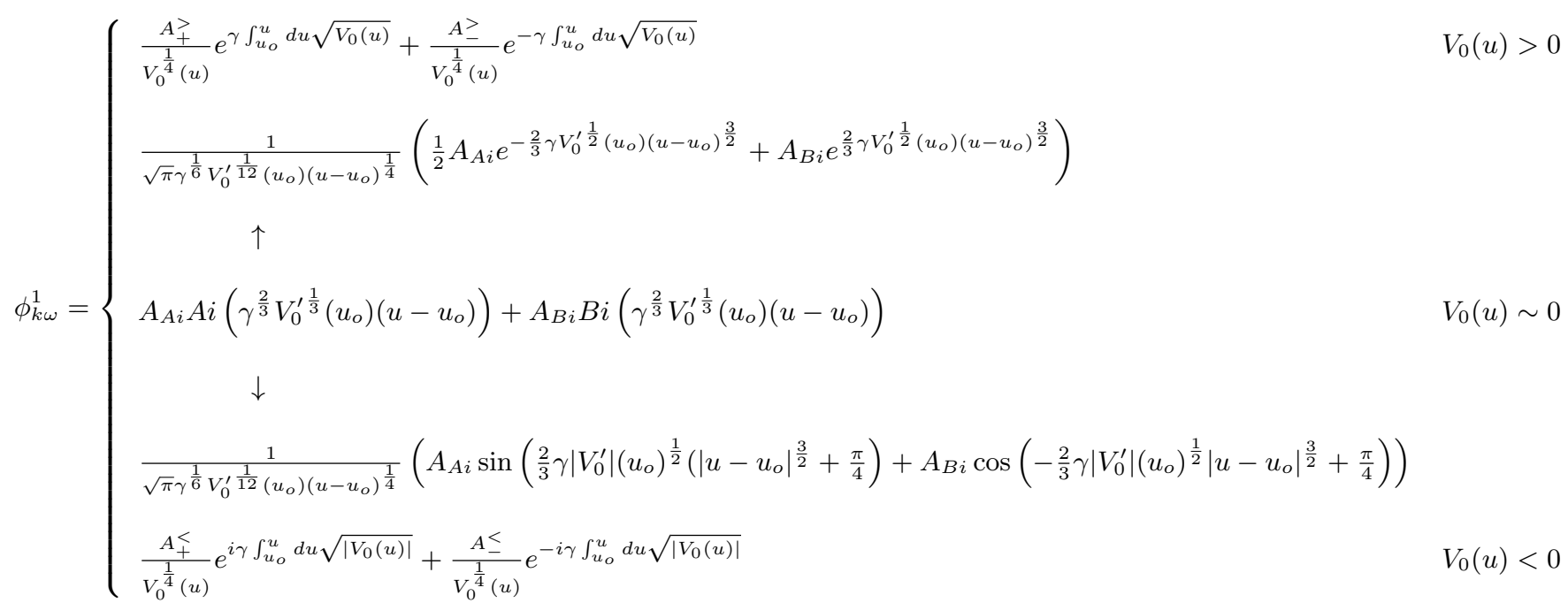

La integral en el exponente de la primera línea puede ser expandida cerca a $u_{o}$ como:

$$
\pm \gamma \int_{u_{o}}^{u} d u \sqrt{\left|V_{0}^{\prime}\left(u_{o}\right)\right|}\left|u-u_{o}\right|^{\frac{1}{2}}=\mp \frac{2}{3} \gamma\left|V_{0}^{\prime}\left(u_{o}\right)\right|^{\frac{1}{2}}\left|u-u_{o}\right|^{\frac{3}{2}} \quad u_{o}<u
$$

Lo mismo es cierto en la última linea:

$$
\pm i \gamma \int_{u_{o}}^{u} d u \sqrt{\left|V_{0}^{\prime}\left(u_{o}\right)\right|}\left|u-u_{o}\right|^{\frac{1}{2}}= \pm \frac{2}{3} i \gamma\left|V_{0}^{\prime}\left(u_{o}\right)\right|^{\frac{1}{2}}\left|u-u_{o}\right|^{\frac{3}{2}} \quad u_{o}>u
$$


Con estos podemos escribir:

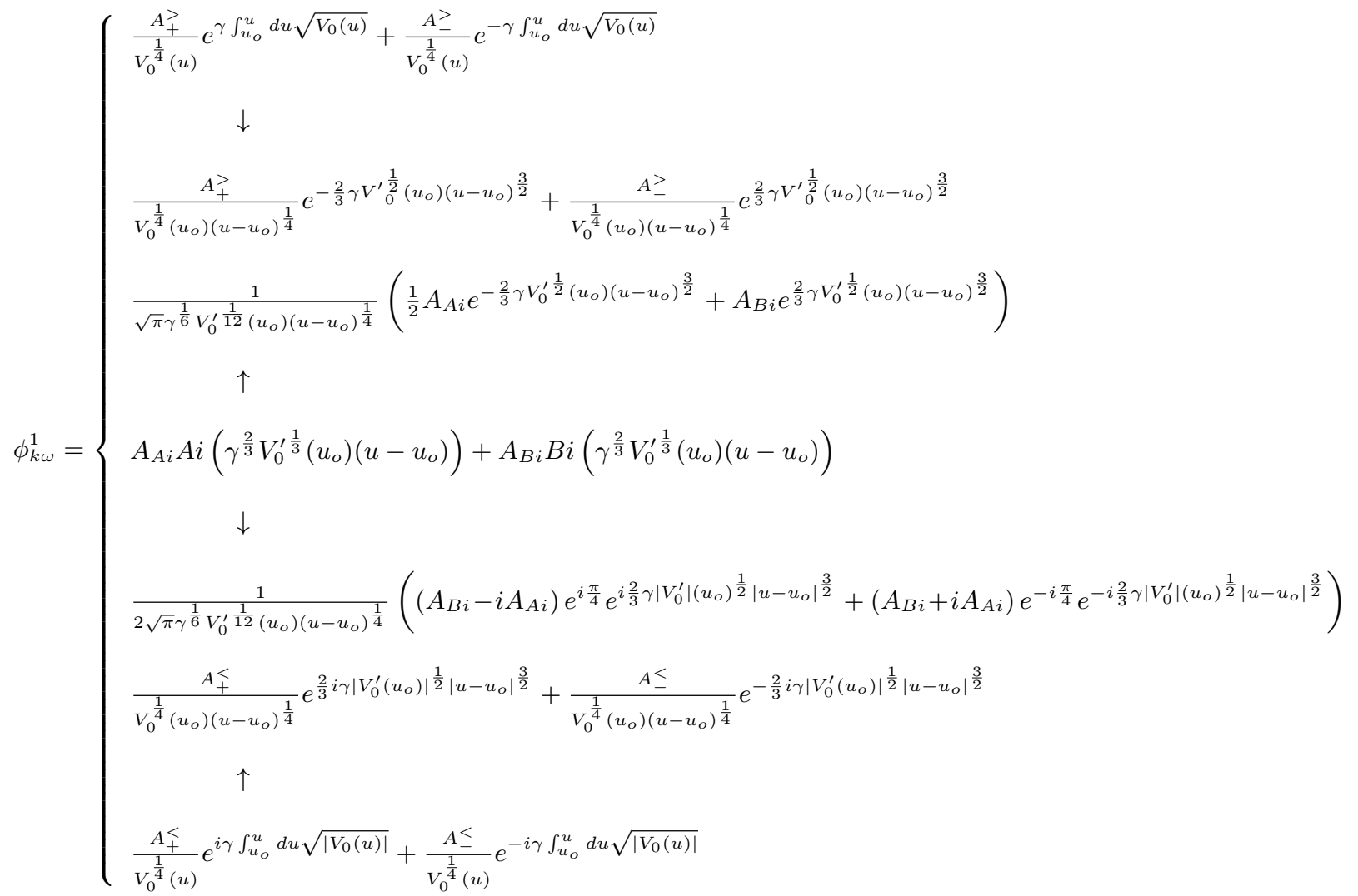

y entonces vemos que:

$$
\begin{gathered}
A_{+}^{>}=\frac{V_{0}^{\frac{1}{4}}\left(u_{o}\right)}{2 \sqrt{\pi} \gamma^{\frac{1}{6}} V_{0}^{\frac{1}{12}}\left(u_{o}\right)} A_{A i} \\
A_{-}^{>}=\frac{V_{0}^{\frac{1}{4}}\left(u_{o}\right)}{\sqrt{\pi} \gamma^{\frac{1}{6}} V_{0}^{\frac{1}{12}}\left(u_{o}\right)} A_{B i} \\
A_{+}^{<}=\frac{V_{0}^{\frac{1}{4}}\left(u_{o}\right)}{2 \sqrt{\pi} \gamma^{\frac{1}{6}} V_{0}^{\prime \frac{1}{12}}\left(u_{o}\right)}\left(A_{B i}-i A_{A i}\right) e^{i \frac{\pi}{4}} \\
A_{-}^{<}=\frac{V_{0}^{\frac{1}{4}}\left(u_{o}\right)}{2 \sqrt{\pi} \gamma^{\frac{1}{6}} V_{0}^{\prime \frac{1}{12}}\left(u_{o}\right)}\left(A_{B i}+i A_{A i}\right) e^{-i \frac{\pi}{4}}
\end{gathered}
$$

Podemos establecer formulas de conexión:

$$
\begin{gathered}
A_{+}^{<}=\left(\frac{1}{2} A_{-}^{>}-i A_{+}^{>}\right) e^{i \frac{\pi}{4}} \\
A_{-}^{<}=\left(\frac{1}{2} A_{-}^{>}+i A_{+}^{>}\right) e^{-i \frac{\pi}{4}}
\end{gathered}
$$

Ahora, para imponer nuestras condiciones de borde, notamos que para $u \rightarrow 0$ tenemos $V_{0} \sim \hat{m}^{2} / u^{2}>0$, entonces estamos en la región $V_{0}>0$, y la solución se escribe como:

$$
\phi_{k \omega}^{1}(z(u))=\frac{A_{+}^{>} \sqrt{u_{o}}}{\sqrt{|\hat{m}|}}\left(\frac{u}{u_{o}}\right)^{\gamma|\hat{m}|+\frac{1}{2}}+\frac{A_{-\sqrt{u_{o}}}^{>}}{\sqrt{|\hat{m}|}}\left(\frac{u}{u_{o}}\right)^{-\gamma|\hat{m}|+\frac{1}{2}}
$$

Dado que el enlace BF para espinores como $\gamma|\hat{m}|=|m| L>\frac{1}{2}$ nuestras condición de borde $\phi_{k_{x} \omega}^{1}(z(0))=0$ entonces implica $A_{-}^{>}=0$. En las formulas de conexión esto implica:

$$
\begin{aligned}
& A_{+}^{<}=-i A_{+}^{>} e^{i \frac{\pi}{4}} \\
& A_{-}^{<}=i A_{+}^{>} e^{-i \frac{\pi}{4}}
\end{aligned}
$$


Entonces la solución se escribe:

$$
\phi_{k \omega}^{1}= \begin{cases}\frac{A_{+}^{>}}{V_{0}^{\frac{1}{4}}(u)} e^{\gamma \int_{u_{o}}^{u} d u \sqrt{V_{0}(u)}} & V_{0}(u)>0 \\ \frac{A_{+}^{>}}{V_{0}^{\frac{1}{4}}(u)} \sin \left(\gamma \int_{u_{o}}^{u} d u \sqrt{\left|V_{0}(u)\right|}+\frac{\pi}{4}\right) & V_{0}(u)<0\end{cases}
$$

Ahora tenemos dos posibilidades: que el potencial tenga un solo punto de retorno y entonces la solución oscilatoria se extiende al HW o si el potencial tiene dos o más puntos de retorno y entonces más conexiones son necesarias. En cualquier caso para satisface las condiciones de borde en el HW $\phi_{k \omega}^{1}\left(z\left(u_{m}\right)\right)=0$ imponemos lo siguiente:

$$
\gamma \int_{u_{o}}^{u_{m}} d u \sqrt{\left|V_{0}(u)\right|}=\left(n-\frac{1}{4}\right) \pi
$$

Esto no es mas que la condición de cuantización de Bohr-Sommerfeld. Notemos que puede ser verificada solo para valores discretos y reales de $\omega=\omega_{n}\left(k_{x}\right)$. En términos de nuestras variables originales $z$ se escribe:

$$
\gamma \int_{z_{o}}^{z_{m}} d z \sqrt{g(z)} \sqrt{\frac{1}{f(z)}(\hat{\omega}+h(z))^{2}-z^{2} \hat{k}^{2}-\hat{m}^{2}}=\left(n-\frac{1}{4}\right) \pi
$$

Entonces los valores correctos de $\omega_{n}(k)$ son seleccionados satisfaciendo estas condiciones. Notemos que $\omega_{n}(k)$ es invariante bajo $k \rightarrow-k$. En consecuencia, las autofunciónes $\phi_{k n}^{1}(z)$ están dadas por:

$$
\phi_{k \omega}^{1}=A_{+}^{>} \times \begin{cases}\frac{\sqrt{\frac{1}{\sqrt{f(z)}}(\hat{\omega}+h(z))+z \hat{k}}}{\left(\hat{m}^{2}-\frac{1}{f(z)}(\hat{\omega}+h(z))^{2}+z^{2} \hat{k}^{2}\right)^{\frac{1}{4}}} e^{\gamma \int_{z_{o}}^{z_{m}} d z \sqrt{g(z)} \sqrt{\hat{m}^{2}-\frac{1}{f(z)}\left(\hat{\omega}_{n}(k)+h(z)\right)^{2}+z^{2} \hat{k}^{2}}} & V_{0}(u)<0 \\ \frac{\sqrt{\frac{1}{\sqrt{f(z)}}(\hat{\omega}+h(z))+z \hat{k}}}{\left(\frac{1}{f(z)}(\hat{\omega}+h(z))^{2}-z^{2} \hat{k}^{2}-\hat{m}^{2}\right)^{\frac{1}{4}}} \sin \left(\gamma \int_{z_{o}}^{z_{m}} d z \sqrt{g(z)} \sqrt{\frac{1}{f(z)}\left(\hat{\omega}_{n}(k)+h(z)\right)^{2}-z^{2} \hat{k}^{2}-\hat{m}^{2}}+\frac{\pi}{4}\right) & V_{0}(u)>0\end{cases}
$$

donde $A_{+}^{>}$es una constante determinada por la normalización.

\subsection{Apéndice D8: interacciones de fermiones en el espacio de fondo holográfico}

Con las consideraciones hechas precedentemente, podemos definir nuestro hamiltoniano libre en el formalismo de la segunda cuantización como:

$$
H_{\text {free }}=\sum_{\alpha n \vec{k}} \omega_{\alpha n}(k) c_{\alpha n \vec{k}}^{\dagger} c_{\alpha n \vec{k}}
$$

Para definir una interacción, notemos que el término de interacción cuártico más general para fermiones de Dirac en un espacio curvo puede escribirse en la forma:

$$
H_{i n t}=\int d^{3} x \sqrt{-g} \sum_{\sigma_{1} \sigma_{2} \sigma_{3} \sigma_{4}} T_{\sigma_{1} \sigma_{2} \sigma_{3} \sigma_{4}} \psi_{\sigma_{1}}^{\dagger} \psi_{\sigma_{2}}^{\dagger} \psi_{\sigma_{3}} \psi_{\sigma_{4}}
$$

donde los índices de espín $\sigma$ 's, corren de 1 a 4 . El tensor invariante $T_{\sigma_{1} \sigma_{2} \sigma_{3} \sigma_{4}}$ está dado por:

$$
\begin{aligned}
T_{\sigma_{1} \sigma_{2} \sigma_{3} \sigma_{4}}= & g_{1}\left(\Gamma^{\underline{t}}\right)_{\sigma_{1} \sigma_{3}}\left(\Gamma^{\underline{t}}\right)_{\sigma_{2} \sigma_{4}}+g_{2}\left(\Gamma^{\underline{t}} \Gamma^{5}\right)_{\sigma_{1} \sigma_{3}}\left(\Gamma^{\underline{t}} \Gamma^{5}\right)_{\sigma_{2} \sigma_{4}}+g_{3}\left(\Gamma^{\underline{t}} \Gamma^{a}\right)_{\sigma_{1} \sigma_{3}}\left(\Gamma^{\underline{t}} \Gamma_{a}\right)_{\sigma_{2} \sigma_{4}}+ \\
& +g_{4}\left(\Gamma^{\underline{t}} \Gamma^{a} \Gamma^{5}\right)_{\sigma_{1} \sigma_{3}}\left(\Gamma^{\underline{t}} \Gamma_{a} \Gamma^{5}\right)_{\sigma_{2} \sigma_{4}}-\frac{1}{4} g_{5}\left(\Gamma^{\underline{t}}\left[\Gamma^{a}, \Gamma^{b}\right] \Gamma^{5}\right)_{\sigma_{1} \sigma_{3}}\left(\Gamma^{\underline{t}}\left[\Gamma_{a}, \Gamma_{b}\right] \Gamma^{5}\right)_{\sigma_{2} \sigma_{4}}
\end{aligned}
$$

este tensor satisface la propiedad de invarianza

$$
T_{\bar{\sigma}_{1} \bar{\sigma}_{2} \bar{\sigma}_{3} \bar{\sigma}_{4}}=T_{\sigma_{1} \sigma_{2} \sigma_{3} \sigma_{4}} D[\theta]_{\sigma^{1} \bar{\sigma}^{1}}^{\dagger} D[\theta]_{\sigma^{2} \bar{\sigma}^{2}}^{\dagger} D[\theta]_{\sigma^{3} \bar{\sigma}^{3}} D[\theta]_{\sigma^{4} \bar{\sigma}^{4}}
$$

donde $D[\theta]$ es la matriz de rotación en el plano $x y$ definida en (503).

Poniendo la solución general en esta interacción obtenemos:

$$
H_{\text {int }}=\sum_{\alpha^{1} n^{1} \vec{k}^{1}} \sum_{\alpha^{2} n^{2} \vec{k}^{2}} \sum_{\alpha^{3} n^{3} \vec{k}^{3}} \sum_{\alpha^{4} n^{3} \vec{k}^{4}} T_{\alpha^{1} n^{1} \vec{k}^{1} \alpha^{2} n^{2} \vec{k}^{2} \alpha^{3} n^{3} \vec{k}^{3} \alpha^{4} n^{4} \vec{k}^{4}} c_{\alpha^{1} n^{1} \vec{k}^{1}}^{\dagger} c_{\alpha^{2} n^{2} \vec{k}^{2}}^{\dagger} c_{\alpha^{3} n^{3} \vec{k}^{3}} c_{\alpha^{4} n^{4} \vec{k}^{4}}
$$


donde:

$$
T_{\alpha^{1} n^{1} \vec{k}^{1} \alpha^{2} n^{2} \vec{k}^{2} \alpha^{3} n^{3} \vec{k}^{3} \alpha^{4} n^{4} \vec{k}^{4}}=T_{\sigma_{1} \sigma_{2} \sigma_{3} \sigma_{4}} \int d x d z \sqrt{-g} \bar{\psi}_{\alpha^{1} n^{1} \vec{k}^{1}}^{\sigma^{1}}(x, z) \bar{\psi}_{\alpha^{2} n^{2} \vec{k}^{2}}^{\sigma^{2}}(x, z) \psi_{\alpha^{3} n^{3} \vec{k}^{3}}^{\sigma^{3}}(x, z) \psi_{\alpha^{4} n^{4} \vec{k}^{4}}^{\sigma^{4}}(x, z)
$$

La integral en $x$ puede ser realizada explícitamente, obteniendo:

$$
T_{\alpha^{1} n^{1} \vec{k}^{1} \alpha^{2} n^{2} \vec{k}^{2} \alpha^{3} n^{3} \vec{k}^{3} \alpha^{4} n^{4} \vec{k}^{4}}=\delta\left(\vec{k}^{1}+\vec{k}^{2}-\vec{k}^{3}-\vec{k}^{4}\right) \hat{T}_{\alpha^{1} n^{1} \vec{k}^{1} \alpha^{2} n^{2} \vec{k}^{2} \alpha^{3} n^{3} \vec{k}^{3} \alpha^{4} n^{4} \vec{k}^{4}}
$$

donde

$$
\begin{aligned}
\hat{T}_{\alpha^{1} n^{1} \vec{k}^{1} \alpha^{2} n^{2} \vec{k}^{2} \alpha^{3} n^{3} \vec{k}^{3} \alpha^{4} n^{4} \vec{k}^{4}}= & T_{\sigma_{1} \sigma_{2} \sigma_{3} \sigma_{4}} D\left[\theta_{1}\right]_{\sigma^{1} \bar{\sigma}^{1}}^{\dagger} D\left[\theta_{2}\right]_{\sigma^{2} \bar{\sigma}^{2}}^{\dagger} D\left[\theta_{3}\right]_{\sigma^{3} \bar{\sigma}^{3}} D\left[\theta_{4}\right]_{\sigma^{4} \bar{\sigma}^{4}} \times \\
& \times \int d z \sqrt{-g} \bar{\psi}_{\alpha^{1} n^{1} k^{1}}^{\bar{\sigma}^{1}}(z) \bar{\psi}_{\alpha^{2} n^{2} k^{2}}^{\bar{\sigma}^{2}}(z) \psi_{\alpha^{3} n^{3} k^{3}}^{\bar{\sigma}^{3}}(z) \psi_{\alpha^{4} n^{4} k^{4}}^{\bar{\sigma}^{4}}(z)
\end{aligned}
$$

donde hemos usado la forma de la solución general. Entonces obtenemos

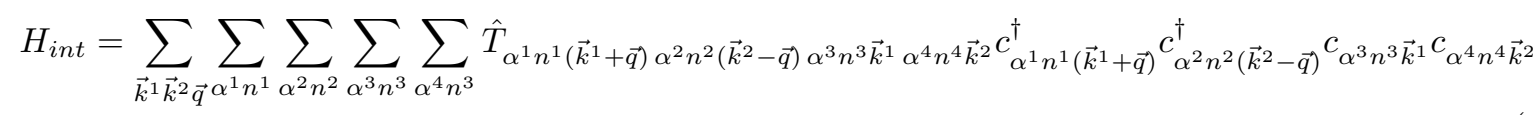

\subsection{Apéndice D9: aproximación de campo medio}

Ahora haremos una análisis de campo medio de la interacción mostrada en apéndice precedente para escribir la correspondiente teoría de Landau. Comenzaremos con nuestro hamiltoniano completo:

$$
H=H_{\text {free }}+H_{\text {int }}
$$

y definimos un hamiltoniano de campo medio como:

$$
H_{M F}=\sum_{\alpha n \vec{k}} \epsilon_{\alpha n}(\vec{k}) c_{\alpha n \vec{k}}^{\dagger} c_{\alpha n \vec{k}}
$$

Siendo la energía libre:

$$
F=-\frac{1}{\beta} \log \operatorname{tr}\left(e^{-\beta H}\right)
$$

podemos escribir:

$$
\begin{aligned}
F & =-\frac{1}{\beta} \log \operatorname{tr}\left(e^{-\beta\left(H-H_{M F}\right)} e^{-\beta H_{M F}}\right)= \\
& =-\frac{1}{\beta} \log \operatorname{tr}\left(e^{-\beta H_{M F}}-\beta\left(H-H_{M F}\right) e^{-\beta H_{M F}}+\cdots\right)= \\
& =-\frac{1}{\beta} \operatorname{tr} \log \left(e^{-\beta H_{M F}}\right)+\frac{\operatorname{tr}\left(\left(H-H_{M F}\right) e^{-\beta H_{M F}}\right)}{\operatorname{tr}\left(e^{-\beta H_{M F}}\right)} \\
& =F_{M F}+\left\langle H-H_{M F}\right\rangle_{M F}+\ldots
\end{aligned}
$$

donde:

$$
\left\langle H-H_{M F}\right\rangle_{M F}=\frac{\operatorname{tr}\left(\left(H-H_{M F}\right) e^{-\beta H_{M F}}\right)}{\operatorname{tr}\left(e^{-\beta H_{M F}}\right)}
$$

La aproximación de campo medio asume que cuando $F$ es minimizada las correcciones en (588) pueden ser descartadas. Para encontrar tal condición escribimos:

$$
\begin{aligned}
& \left\langle H-H_{M F}\right\rangle_{M F}=\sum_{\alpha n \vec{k}}\left(\omega_{\alpha n}(k)-\epsilon_{\alpha n}(\vec{k})\right)\left\langle c_{\alpha n \vec{k}}^{\dagger} c_{\alpha n \vec{k}}\right\rangle_{M F} \\
& +\sum_{\vec{k}^{1} \vec{k}^{2} \vec{q}^{\alpha^{1} n^{1}}} \sum_{\alpha^{2} n^{2}} \sum_{\alpha^{3} n^{3}} \sum_{\alpha^{4} n^{4}} \hat{T}_{\alpha^{1} n^{1}\left(\vec{k}^{1}+\vec{q}\right) \alpha^{2} n^{2}\left(\vec{k}^{2}-\vec{q}\right) \alpha^{3} n^{3} \vec{k}^{1} \alpha^{4} n^{4} \vec{k}^{2}}\left\langle c_{\alpha^{1} n^{1}\left(\vec{k}^{1}+\vec{q}\right)}^{\dagger} c_{\alpha^{2} n^{2}\left(\vec{k}^{2}-\vec{q}\right)}^{\dagger} c_{\alpha^{3} n^{3} \vec{k}^{1}} c_{\alpha^{4} n^{4} \vec{k}^{2}}\right\rangle_{M F}
\end{aligned}
$$


Usando el teorema de Wick podemos escribir:

$$
\begin{aligned}
& \left\langle c_{\alpha^{1} n^{1}\left(\vec{k}^{1}+\vec{q}\right)}^{\dagger} c_{\alpha^{2} n^{2}\left(\vec{k}^{2}-\vec{q}\right)}^{\dagger} c_{\alpha^{3} n^{3} \vec{k}^{1}} c_{\alpha^{4} n^{4} \vec{k}^{2}}\right\rangle_{M F}=\left\langle c_{\alpha^{1} n^{1}\left(\vec{k}^{1}+\vec{q}\right)}^{\dagger} c_{\alpha^{4} n^{4} \vec{k}^{2}}\right\rangle_{M F}\left\langle c_{\alpha^{2} n^{2}\left(\vec{k}^{2}-\vec{q}^{2}\right)}^{\dagger} c_{\alpha^{3} n^{3} \vec{k}^{1}}\right\rangle_{M F} \\
& -\left\langle c_{\alpha^{1} n^{1}\left(\vec{k}^{1}+\vec{q}\right)}^{\dagger} c_{\alpha^{3} n^{3} \vec{k}^{1}}\right\rangle_{M F}\left\langle c_{\alpha^{2} n^{2}\left(\vec{k}^{2}-\vec{q}\right)}^{\dagger} c_{\alpha^{4} n^{4} \vec{k}^{2}}\right\rangle_{M F} \\
= & \delta_{\alpha^{1} \alpha^{4}} \delta_{n^{1} n^{4}} \delta\left(\vec{k}^{1}+\vec{q}-\vec{k}^{2}\right) N_{\alpha^{1} n^{1}\left(\vec{k}^{1}+\vec{q}\right)} \delta_{\alpha_{2} \alpha_{3}} \delta_{n^{2} n^{3}} \delta\left(\vec{k}^{2}-\vec{q}-\vec{k}^{1}\right) N_{\alpha^{2} n^{2}\left(\vec{k}^{2}-\vec{q}\right)} \\
& -\delta_{\alpha^{1} \alpha^{3}} \delta_{n^{1} n^{3}} \delta(\vec{q}) N_{\alpha^{1} n^{1} \vec{k}^{1}} \delta_{\alpha^{2} \alpha^{4}} \delta_{n^{2} n^{4}} \delta(\vec{q}) N_{\alpha^{2} n^{2} \vec{k}^{2}} \\
= & \left(\delta_{\alpha^{1} \alpha^{4}} \delta_{\alpha^{2} \alpha^{3}} \delta_{n^{1} n^{4}} \delta_{n^{2} n^{3}} \delta^{2}\left(\vec{k}^{1}+\vec{q}-\vec{k}^{2}\right)-\delta_{\alpha^{1} \alpha^{3}} \delta_{\alpha^{2} \alpha^{4}} \delta_{n^{1} n^{3}} \delta_{n^{2} n^{4}} \delta^{2}(\vec{q})\right) \times \\
\times & N_{\alpha^{1} n^{1}\left(\vec{k}^{1}+\vec{q}\right)} N_{\alpha^{2} n^{2}\left(\vec{k}^{2}-\vec{q}\right)}
\end{aligned}
$$

Por lo tanto tenemos que:

$$
\begin{aligned}
& \left\langle H-H_{M F}\right\rangle_{M F}=\sum_{\alpha n \vec{k}}\left(\omega_{\alpha n}(k)-\epsilon_{\alpha n}(\vec{k})\right) N_{\alpha n \vec{k}} \\
+ & \sum_{\vec{k}^{1} \vec{k}^{2} \vec{q}^{\alpha^{1} n^{1}}} \sum_{\alpha^{2} n^{2}} \sum_{\alpha^{3} n^{3}} \sum_{\alpha^{4} n^{4}} \hat{T}_{\alpha^{1} n^{1}\left(\vec{k}^{1}+\vec{q}\right) \alpha^{2} n^{2}\left(\vec{k}^{2}-\vec{q}\right) \alpha^{3} n^{3} \vec{k}^{1} \alpha^{4} n^{4} \vec{k}^{2}} \\
& \left(\delta_{\alpha^{1} \alpha^{4}} \delta_{\alpha^{2} \alpha^{3}} \delta_{n^{1} n^{4}} \delta_{n^{2} n^{3}} \delta^{2}\left(\vec{k}^{1}+\vec{q}-\vec{k}^{2}\right)-\delta_{\alpha^{1} \alpha^{3}} \delta_{\alpha^{2} \alpha^{4}} \delta_{n^{1} n^{3}} \delta_{n^{2} n^{4}} \delta^{2}(\vec{q})\right) N_{\alpha^{1} n^{1} \vec{k}^{1}+\vec{q}^{2}} N_{\alpha^{2} n^{2} \vec{k}^{2}-\vec{q}}
\end{aligned}
$$

Usando las funciónes delta para colapsar las sumas (notemos que podemos regularizar la $\delta^{2}$ en índices de momentos que aparece en la suma de arriba imponiendo un volumen finito al espacio $\vec{x}$ y entonces tomar el límite de volumen yendo a infinito al final de los cálculos) obtenemos:

$$
\left\langle H-H_{M F}\right\rangle_{M F}=\sum_{\alpha n \vec{k}}\left(\omega_{\alpha n}(k)-\epsilon_{\alpha n}(\vec{k})\right) N_{\alpha n \vec{k}}+\frac{1}{2} \sum_{\vec{k}^{1} \vec{k}^{2}} \sum_{\alpha^{1} n^{1}} \sum_{\alpha^{2} n^{2}} f_{\alpha^{1} n^{1} \alpha^{2} n^{2}}\left(\vec{k}^{1}, \vec{k}^{2}\right) N_{\alpha^{1} n^{1} \vec{k}^{1}} N_{\alpha^{2} n^{2} \vec{k}^{2}}
$$

donde:

$$
f_{\alpha^{1} n^{1} \alpha^{2} n^{2}}\left(\vec{k}^{1}, \vec{k}^{2}\right)=2 \hat{T}_{\alpha^{1} n^{1} \vec{k}^{1} \alpha^{2} n^{2} \vec{k}^{2} \alpha^{2} n^{2} \vec{k}^{2} \alpha^{1} n^{1} \vec{k}^{1}}-2 \hat{T}_{\alpha^{1} n^{1} \vec{k}^{1} \alpha^{2} n^{2} \vec{k}^{2} \alpha^{1} n^{1} \vec{k}^{1} \alpha^{2} n^{2} \vec{k}^{2}}
$$

Como $F_{M F}=-\frac{1}{\beta} \log \left[Z_{M F}\right]$ y para sistemas fermiónicos a bajas temperaturas podemos escribir $Z_{M F}=$ $\prod_{\alpha n \vec{k}} Z_{\alpha n \vec{k}}$ con:

$$
Z_{\alpha n \vec{k}}=\sum_{N_{\alpha n \vec{k}}=0}^{1} e^{-\beta N_{\alpha n \vec{k}}\left(\epsilon_{\alpha n}(\vec{k})-\mu\right)}=1+e^{-\beta\left(\epsilon_{\alpha n}(\vec{k})-\mu\right)}
$$

tenemos:

$$
F_{M F}=-\frac{1}{\beta} \log \left(\prod_{\alpha n \vec{k}}\left(1+e^{-\beta \epsilon_{\alpha n}(\vec{k})}\right)\right)=-\frac{1}{\beta} \sum_{\alpha n \vec{k}} \log \left(1+e^{-\beta \epsilon_{\alpha n}(\vec{k})}\right)
$$

Entonces, dentro de nuestra aproximación la energía libre es:

$$
\begin{aligned}
F & =\sum_{\alpha n \vec{k}}\left(\omega_{\alpha n}(k)-\epsilon_{\alpha n}(\vec{k})\right) N_{\alpha n \vec{k}}+\frac{1}{2} \sum_{\vec{k}^{1} \vec{k}^{2}} \sum_{\alpha^{1} n^{1}} \sum_{\alpha^{2} n^{2}} f_{\alpha^{1} n^{1} \alpha^{2} n^{2}}\left(\vec{k}^{1}, \vec{k}^{2}\right) N_{\alpha^{1} n^{1} \vec{k}^{1}} N_{\alpha^{2} n^{2} \vec{k}^{2}} \\
& -\frac{1}{\beta} \sum_{\alpha n \vec{k}} \log \left(1+e^{-\beta \epsilon_{\alpha n}(\vec{k})}\right)
\end{aligned}
$$

donde $\epsilon_{\alpha n}(\vec{k})$ y $N_{\alpha n \vec{k}}$ son tales que minimizan $F$ y por lo tanto satisfacen las ecuaciones de auto-consistencia:

$$
\epsilon_{\alpha n}(\vec{k})=\omega_{\alpha n}(k)+\sum_{\vec{k}^{1}} \sum_{\alpha^{1} n^{1}} f_{\alpha n \alpha^{1} n^{1}}\left(\vec{k}, \vec{k}^{1}\right) N_{\alpha^{1} n^{1} \vec{k}^{1}}
$$

$\mathrm{y}$

$$
N_{\alpha n \vec{k}}=\frac{1}{1+e^{-\beta \epsilon_{\alpha n}(\vec{k})}}
$$

Ahora para simplificar $f_{\alpha^{1} n^{1} \alpha^{2} n^{2}}\left(\vec{k}^{1}, \vec{k}^{2}\right)$ comenzamos escribiendo:

$$
\begin{aligned}
\hat{T}_{\alpha^{1} n^{1} \vec{k}^{1} \alpha^{2} n^{2} \vec{k}^{2} \alpha^{3} n^{3} \vec{k}^{3} \alpha^{4} n^{4} \vec{k}^{4}} & =T_{\sigma_{1} \sigma_{2} \sigma_{3} \sigma_{4}} D\left[\theta_{1}\right]_{\sigma^{1} \bar{\sigma}^{1}}^{\dagger} D\left[\theta_{2}\right]_{\sigma^{2} \bar{\sigma}^{2}}^{\dagger} D\left[\theta_{1}\right]_{\sigma^{3} \bar{\sigma}^{3}} D\left[\theta_{2}\right]_{\sigma^{4} \bar{\sigma}^{4}} \times \\
& \times \int d z \sqrt{-g} \bar{\psi}_{\alpha^{1} n^{1} k^{1}}^{\bar{\sigma}^{1}}(z) \bar{\psi}_{\alpha^{2} n^{2} k^{2}}(z) \psi_{\alpha^{3} n^{3} k^{3}}(z) \psi_{\alpha^{4} n^{4} k^{4}}(z)
\end{aligned}
$$


Luego usamos la propiedad de invarianza para escribir:

$$
\begin{aligned}
\hat{T}_{\alpha^{1} n^{1} \vec{k}^{1} \alpha^{2} n^{2} \vec{k}^{2} \alpha^{3} n^{3} \vec{k}^{1} \alpha^{4} n^{4} \vec{k}^{2}} & =T_{\bar{\sigma}_{1} \sigma_{2} \bar{\sigma}_{3} \sigma_{4}} D\left[\theta_{2}-\theta_{1}\right]_{\sigma^{2} \bar{\sigma}^{2}}^{\dagger} D\left[\theta_{2}-\theta_{1}\right]_{\sigma^{4} \bar{\sigma}^{4}} \times \\
& \times \int d z \sqrt{-g} \bar{\psi}_{\alpha^{1} n^{1} k^{1}}^{\bar{\sigma}^{1}}(z) \bar{\psi}_{\alpha^{2} n^{2} k^{2}}(z) \psi_{\alpha^{3} n^{3} k^{3}}(z) \psi_{\alpha^{4} n^{4} k^{4}}^{\bar{\sigma}^{4}}(z)
\end{aligned}
$$

implicando:

$$
f_{\alpha^{1} n^{1} \alpha^{2} n^{2}}\left(\vec{k}^{1}, \vec{k}^{2}\right)=\sum_{\sigma_{1} \sigma_{2} \sigma_{3} \sigma_{4}} 2 T_{\bar{\sigma}_{1} \bar{\sigma}_{2} \bar{\sigma}_{3} \bar{\sigma}_{4}}\left(\theta_{2}-\theta_{1}\right) \int d z \sqrt{-g} \bar{\psi}_{\alpha^{1} n^{1} k^{1}}^{\sigma^{1}}(z) \bar{\psi}_{\alpha^{2} n^{2} k^{2}}^{\sigma^{2}}(z) \psi_{\alpha^{2} n^{2} k^{2}}^{\sigma^{3}}(z) \psi_{\alpha^{1} n^{1} k^{1}}^{\sigma^{4}}(z)
$$

donde la integral muestra independencia angular y definimos un tensor con dependencia angular como

$$
T_{\sigma_{1} \sigma_{2} \sigma_{3} \sigma_{4}}(\theta)=\sum_{\bar{\sigma}_{2} \bar{\sigma}_{4}}\left(T_{\sigma_{1} \bar{\sigma}_{2} \sigma_{3} \bar{\sigma}_{4}} D[\theta]_{\bar{\sigma}^{2} \sigma^{2}}^{\dagger} D[\theta]_{\bar{\sigma}^{4} \sigma^{4}}-T_{\sigma_{1} \bar{\sigma}_{2} \sigma_{4} \bar{\sigma}_{4}} D[\theta]_{\bar{\sigma}^{2} \sigma^{2}}^{\dagger} D[\theta]_{\bar{\sigma}^{4} \sigma^{3}}\right)
$$

El tensor que depende del ángulo puede ser escrito como:

$$
\begin{aligned}
& T_{\sigma_{1} \sigma_{2} \sigma_{3} \sigma_{4}}(\theta)=\sum_{\bar{\sigma}_{2} \bar{\sigma}_{4}}\left(\left(T_{\sigma_{1} \sigma_{2} \sigma_{3} \sigma_{4}}-T_{\sigma_{1} \sigma_{2} \sigma_{4} \sigma_{3}}\right) \cos ^{2} \frac{\theta}{2}+\left(T_{\sigma_{1} \bar{\sigma}_{2} \sigma_{4} \bar{\sigma}_{4}} \Gamma_{\bar{\sigma}^{4} \sigma^{3}}^{\underline{x y}}-T_{\sigma_{1} \bar{\sigma}_{2} \sigma_{3} \bar{\sigma}_{4}} \Gamma_{\bar{\sigma}^{4} \sigma^{4}}^{\underline{x y}}\right) \Gamma_{\bar{\sigma}^{2}}^{\underline{x} \sigma^{2}} \sin ^{2} \frac{\theta}{2}\right. \\
& \left.+\left(T_{\sigma_{1} \sigma_{2} \sigma_{3} \bar{\sigma}_{4}} \Gamma_{\bar{\sigma}^{4} \sigma^{4}}^{\underline{x} \underline{y}}-T_{\sigma_{1} \sigma_{2} \sigma_{4} \bar{\sigma}_{4}} \Gamma_{\bar{\sigma}^{4} \sigma^{3}}^{\underline{x y}}+\left(T_{\sigma_{1} \bar{\sigma}_{2} \sigma_{4} \sigma_{3}}-T_{\sigma_{1} \bar{\sigma}_{2} \sigma_{3} \sigma_{4}}\right) \Gamma_{\bar{\sigma}^{2}}^{\underline{x} \sigma^{2}}\right) \cos \frac{\theta}{2} \sin \frac{\theta}{2}\right) \\
& =\frac{1}{2} \sum_{\bar{\sigma}_{2} \bar{\sigma}_{4}}\left(\left(T_{\sigma_{1} \sigma_{2} \sigma_{3} \sigma_{4}}-T_{\sigma_{1} \sigma_{2} \sigma_{4} \sigma_{3}}\right)(1+\cos \theta)+\left(T_{\sigma_{1} \bar{\sigma}_{2} \sigma_{4} \bar{\sigma}_{4}} \Gamma_{\bar{\sigma}^{4} \sigma^{3}}^{\underline{x y}}-T_{\sigma_{1} \bar{\sigma}_{2} \sigma_{3} \bar{\sigma}_{4}} \Gamma_{\bar{\sigma}^{4}}^{\underline{x} \sigma^{4}}\right) \Gamma_{\bar{\sigma}^{2}}^{\underline{x} \sigma^{2}}(1-\cos \theta)\right. \\
& \left.+\left(T_{\sigma_{1} \sigma_{2} \sigma_{3} \bar{\sigma}_{4}} \Gamma_{\bar{\sigma}^{4} \sigma^{4}}^{\underline{x y}}-T_{\sigma_{1} \sigma_{2} \sigma_{4} \bar{\sigma}_{4}} \Gamma_{\bar{\sigma}^{4} \sigma^{3}}^{\underline{x y}}+\left(T_{\sigma_{1} \bar{\sigma}_{2} \sigma_{4} \sigma_{3}}-T_{\sigma_{1} \bar{\sigma}_{2} \sigma_{3} \sigma_{4}}\right) \Gamma_{\bar{\sigma}^{2}}^{\underline{x} \sigma^{2}}\right) \sin \theta\right) \\
& =\frac{1}{2} \sum_{\bar{\sigma}_{2} \bar{\sigma}_{4}}\left(T_{\sigma_{1} \sigma_{2} \sigma_{3} \sigma_{4}}-T_{\sigma_{1} \sigma_{2} \sigma_{4} \sigma_{3}}+\left(T_{\sigma_{1} \bar{\sigma}_{2} \sigma_{4} \bar{\sigma}_{4}} \Gamma_{\bar{\sigma}^{4}}^{\underline{x y} \sigma^{3}}-T_{\sigma_{1} \bar{\sigma}_{2} \sigma_{3} \bar{\sigma}_{4}} \Gamma_{\bar{\sigma}^{4}}^{\underline{x} \sigma^{4}}\right) \Gamma_{\bar{\sigma}^{2}}^{\underline{\underline{x}} \sigma^{2}}\right. \\
& +\left(T_{\sigma_{1} \sigma_{2} \sigma_{3} \sigma_{4}}-T_{\sigma_{1} \sigma_{2} \sigma_{4} \sigma_{3}}-\left(T_{\sigma_{1} \bar{\sigma}_{2} \sigma_{4} \bar{\sigma}_{4}} \Gamma_{\bar{\sigma}^{4} \sigma^{3}}^{\underline{x} y}-T_{\sigma_{1} \bar{\sigma}_{2} \sigma_{3} \bar{\sigma}_{4}} \Gamma_{\bar{\sigma}^{4} \sigma^{4}}^{\underline{x} y}\right) \Gamma_{\bar{\sigma}^{2}}^{\underline{x}} \sigma^{2}\right) \cos \theta \\
& \left.+\left(T_{\sigma_{1} \sigma_{2} \sigma_{3} \bar{\sigma}_{4}} \Gamma_{\bar{\sigma}^{4} \sigma^{4}}^{\underline{x}}-T_{\sigma_{1} \sigma_{2} \sigma_{4} \bar{\sigma}_{4}} \Gamma_{\bar{\sigma}^{4} \sigma^{3}}^{\underline{x}}+\left(T_{\sigma_{1} \bar{\sigma}_{2} \sigma_{4} \sigma_{3}}-T_{\sigma_{1} \bar{\sigma}_{2} \sigma_{3} \sigma_{4}}\right) \Gamma_{\bar{\sigma}^{2}}^{\underline{x} \sigma^{2}}\right) \sin \theta\right)
\end{aligned}
$$

implicando que:

$$
f_{\alpha^{1} n^{1} \alpha^{2} n^{2}}\left(\vec{k}^{1}, \vec{k}^{2}\right)=f_{\alpha^{1} n^{1} \alpha^{2} n^{2}}^{1}\left(k^{1}, k^{2}\right)+f_{\alpha^{1} n^{1} \alpha^{2} n^{2}}^{c}\left(k^{1}, k^{2}\right) \cos \left(\theta_{2}-\theta_{1}\right)+f_{\alpha^{1} n^{1} \alpha^{2} n^{2}}^{s}\left(k^{1}, k^{2}\right) \sin \left(\theta_{2}-\theta_{1}\right)
$$

con:

$$
\begin{aligned}
f_{\alpha^{1} n^{1} \alpha^{2} n^{2}}^{1}\left(k^{1}, k^{2}\right)= & \sum_{\sigma_{1} \sigma_{2} \sigma_{3} \sigma_{4} \bar{\sigma}_{2} \bar{\sigma}_{4}}\left(T_{\sigma_{1} \sigma_{2} \sigma_{3} \sigma_{4}}-T_{\sigma_{1} \sigma_{2} \sigma_{4} \sigma_{3}}+\left(T_{\sigma_{1} \bar{\sigma}_{2} \sigma_{4} \bar{\sigma}_{4}} \Gamma_{\bar{\sigma}^{4} \sigma^{3}}^{\underline{x y}}-T_{\sigma_{1} \bar{\sigma}_{2} \sigma_{3} \bar{\sigma}_{4}} \Gamma_{\bar{\sigma}^{4} \sigma^{4}}^{\underline{x} y}\right) \Gamma_{\bar{\sigma}^{2}}^{\underline{\underline{x}} \sigma^{2}}\right) \times \\
& \times \int d z \sqrt{-g} \bar{\psi}_{\alpha^{1} n^{1} k^{1}}^{\sigma^{1}}(z) \bar{\psi}_{\alpha^{2} n^{2} k^{2}}^{\sigma^{2}}(z) \psi_{\alpha^{2} n^{2} k^{2}}^{\sigma^{3}}(z) \psi_{\alpha^{1} n^{1} k^{1}}^{\sigma^{4}}(z)
\end{aligned}
$$$$
f_{\alpha^{1} n^{1} \alpha^{2} n^{2}}^{c}\left(k^{1}, k^{2}\right)=\sum_{\sigma_{1} \sigma_{2} \sigma_{3} \sigma_{4} \bar{\sigma}_{2} \bar{\sigma}_{4}}\left(T_{\sigma_{1} \sigma_{2} \sigma_{3} \sigma_{4}}-T_{\sigma_{1} \sigma_{2} \sigma_{4} \sigma_{3}}-\left(T_{\sigma_{1} \bar{\sigma}_{2} \sigma_{4} \bar{\sigma}_{4}} \Gamma_{\bar{\sigma}^{4} \sigma^{3}}-T_{\sigma_{1} \bar{\sigma}_{2} \sigma_{3} \bar{\sigma}_{4}} \Gamma_{\bar{\sigma}^{4} \sigma^{4}}\right) \Gamma_{\bar{\sigma}^{2} \sigma^{2}}\right) \times
$$$$
\times \int d z \sqrt{-g} \bar{\psi}_{\alpha^{1} n^{1} k^{1}}^{\sigma^{1}}(z) \bar{\psi}_{\alpha^{2} n^{2} k^{2}}^{\sigma^{2}}(z) \psi_{\alpha^{2} n^{2} k^{2}}^{\sigma^{3}}(z) \psi_{\alpha^{1} n^{1} k^{1}}^{\sigma^{4}}(z)
$$

$\mathrm{y}$

$$
\begin{aligned}
f_{\alpha^{1} n^{1} \alpha^{2} n^{2}}^{s}\left(k^{1}, k^{2}\right)= & \sum_{\sigma_{1} \sigma_{2} \sigma_{3} \sigma_{4} \bar{\sigma}_{2} \bar{\sigma}_{4}}\left(T_{\sigma_{1} \sigma_{2} \sigma_{3} \bar{\sigma}_{4}} \Gamma_{\bar{\sigma}^{4} \sigma^{4}}^{\underline{x y}}-T_{\sigma_{1} \sigma_{2} \sigma_{4} \bar{\sigma}_{3}} \Gamma_{\bar{\sigma}^{3} \sigma^{3}}^{\underline{x} y}+\left(T_{\sigma_{1} \bar{\sigma}_{2} \sigma_{4} \sigma_{3}}-T_{\sigma_{1} \bar{\sigma}_{2} \sigma_{3} \sigma_{4}}\right) \Gamma_{\bar{\sigma}^{2} \sigma^{2}}^{\underline{x y}}\right) \times \\
& \times \int d z \sqrt{-g} \bar{\psi}_{\alpha^{1} n^{1} k^{1}}^{\sigma^{1}}(z) \bar{\psi}_{\alpha^{2} n^{2} k^{2}}^{\sigma^{2}}(z) \psi_{\alpha^{2} n^{2} k^{2}}^{\sigma^{3}}(z) \psi_{\alpha^{1} n^{1} k^{1}}^{\sigma^{4}}(z)
\end{aligned}
$$

Con todo esto podemos escribir la fórmula de Landau:

$$
\delta F=\sum_{n \alpha} \int d^{2} k \epsilon_{\alpha n}(\vec{k}) \delta N_{\alpha n}(\vec{k})+\frac{1}{2} \sum_{n \alpha n^{\prime} \alpha^{\prime}} \int d^{2} k \int d^{2} k^{\prime} f_{\alpha n \alpha^{\prime} n^{\prime}}\left(\vec{k}, \vec{k}^{\prime}\right) \delta N_{\alpha n}(\vec{k}) \delta N_{\alpha^{\prime} n^{\prime}}\left(\vec{k}^{\prime}\right)
$$




\subsection{Apéndice D10: construcción de Pomeranchuk}

Usando la descomposición:

$$
\begin{aligned}
\delta N_{\alpha n}(\vec{k}) & =H\left[-\epsilon_{\alpha n}(\vec{k})+\delta_{\alpha n}(\vec{k})\right]-H\left[-\epsilon_{\alpha n}(\vec{k})\right]= \\
& \simeq H^{\prime}\left[-\epsilon_{\alpha n}(\vec{k})\right] \delta_{\alpha n}(\vec{k})+\frac{1}{2} H^{\prime \prime}\left[-\epsilon_{\alpha n}(\vec{k})\right] \delta_{\alpha n}(\vec{k}) \delta_{\alpha^{\prime} n^{\prime}}\left(\vec{k}^{\prime}\right) \\
& =\delta\left[-\epsilon_{\alpha n}(\vec{k})\right] \delta_{\alpha n}(\vec{k})+\frac{1}{2} \delta^{\prime}\left[-\epsilon_{\alpha n}(\vec{k})\right] \delta_{\alpha n}(\vec{k}) \delta_{\alpha^{\prime} n^{\prime}}\left(\vec{k}^{\prime}\right)
\end{aligned}
$$

podemos escribir la fórmula de Landau como:

$$
\begin{aligned}
\delta F & \simeq \frac{1}{2} \sum_{n \alpha n^{\prime} \alpha^{\prime}} \int d^{2} k \int d^{2} k^{\prime}\left(\epsilon_{\alpha n}(\vec{k}) \delta^{\prime}\left[-\epsilon_{\alpha n}(\vec{k})\right] \delta_{\alpha n \alpha^{\prime} n^{\prime}} \delta\left(\vec{k}-\vec{k}^{\prime}\right)+f_{\alpha n \alpha^{\prime} n^{\prime}}\left(\vec{k}, \vec{k}^{\prime}\right) \delta\left[-\epsilon_{\alpha n}(\vec{k})\right] \delta\left[-\epsilon_{\alpha^{\prime} n^{\prime}}\left(\vec{k}^{\prime}\right)\right]\right) \times \\
& \times \delta_{\alpha n}(\vec{k}) \delta_{\alpha^{\prime} n^{\prime}}\left(\vec{k}^{\prime}\right)
\end{aligned}
$$

Usando invarianza rotacional y coordenadas polares en el espacio de momentos obtenemos:

$$
\begin{aligned}
& \delta F \simeq \frac{1}{2} \sum_{n \alpha n^{\prime} \alpha^{\prime}} \int d k d \theta \int d k^{\prime} d \theta^{\prime}\left(k \epsilon_{\alpha n}(k) \delta^{\prime}\left[-\epsilon_{\alpha n}(\vec{k})\right] \delta_{\alpha n \alpha^{\prime} n^{\prime}} \delta\left(\vec{k}-\vec{k}^{\prime}\right)+\right. \\
&\left.\quad+k k^{\prime} f_{\alpha n \alpha^{\prime} n^{\prime}}\left(k, k^{\prime}, \theta-\theta^{\prime}\right) \delta\left[-\epsilon_{\alpha n}(k)\right] \delta\left[-\epsilon_{\alpha^{\prime} n^{\prime}}\left(k^{\prime}\right)\right]\right) \delta_{\alpha n}(\vec{k}) \delta_{\alpha^{\prime} n^{\prime}}\left(\vec{k}^{\prime}\right) \\
&=\frac{1}{2} \sum_{n \alpha n^{\prime} \alpha^{\prime}} \int d k d \theta \int d k^{\prime} d \theta^{\prime}\left(k_{F}^{\alpha n} \delta_{\alpha n \alpha^{\prime} n^{\prime}} \delta\left(\theta-\theta^{\prime}\right)+k_{F}^{\alpha n} k_{F}^{\alpha^{\prime} n^{\prime}} f_{\alpha n \alpha^{\prime} n^{\prime}}\left(k_{F}^{\alpha n}, k_{F}^{\alpha^{\prime} n^{\prime}}, \theta-\theta^{\prime}\right)\right) \delta_{\alpha n}(\theta) \delta_{\alpha^{\prime} n^{\prime}}\left(\theta^{\prime}\right)
\end{aligned}
$$

donde el momento de Fermi $k_{F}^{\alpha n}$ está localizado en los ceros de $\epsilon_{\alpha n}(k)$. Ahora descomponiendo las deformaciones como:

$$
\delta_{\alpha n}(\theta)=c_{\alpha n}^{1}+c_{\alpha n}^{c} \cos \theta+c_{\alpha n}^{s} \sin \theta
$$

obtenemos:

$$
\begin{aligned}
\delta F \simeq \sum_{n \alpha n^{\prime} \alpha^{\prime}} & \frac{\pi}{2} \\
& \left(2 k_{F}^{\alpha n}\left(\delta_{\alpha n \alpha^{\prime} n^{\prime}}+2 \pi k_{F}^{\alpha^{\prime} n^{\prime}} f_{\alpha n \alpha^{\prime} n^{\prime}}^{1}\left(k_{F}^{\alpha n}, k_{F}^{\alpha^{\prime} n^{\prime}}\right)\right) c_{\alpha n}^{1} c_{\alpha^{\prime} n^{\prime}}^{1}+\right. \\
& +k_{F}^{\alpha n}\left(\delta_{\alpha n \alpha^{\prime} n^{\prime}}+\pi k_{F}^{\alpha^{\prime} n^{\prime}} f_{\alpha n \alpha^{\prime} n^{\prime}}^{c}\left(k_{F}^{\alpha n}, k_{F}^{\alpha^{\prime} n^{\prime}}\right)\right)\left(c_{\alpha n}^{c} c_{\alpha^{\prime} n^{\prime}}^{c}+c_{\alpha n}^{s} c_{\alpha^{\prime} n^{\prime}}^{s}\right)+ \\
& \left.+\pi k_{F}^{\alpha n} k_{F}^{\alpha^{\prime} n^{\prime}}\left(f_{\alpha n \alpha^{\prime} n^{\prime}}^{s}\left(k_{F}^{\alpha n}, k_{F}^{\alpha^{\prime} n^{\prime}}\right)-f_{\alpha^{\prime} n^{\prime} \alpha n}^{s}\left(k_{F}^{\alpha n}, k_{F}^{\alpha^{\prime} n^{\prime}}\right)\right) c_{\alpha n}^{c} c_{\alpha^{\prime} n^{\prime}}^{s}\right)
\end{aligned}
$$

Esto implica que para tener una energía definida positiva, la forma cuadrática debe ser positiva. En otras palabras, obtenemos una condición sobre los menores de las matrices:

$$
\begin{array}{r}
\forall N: \quad\left|k_{F}^{\alpha n}\left(\delta_{\alpha n \alpha^{\prime} n^{\prime}}+2 \pi k_{F}^{\alpha^{\prime} n^{\prime}} f_{\alpha n \alpha^{\prime} n^{\prime}}^{1}\left(k_{F}^{\alpha n}, k_{F}^{\alpha^{\prime} n^{\prime}}\right)\right)\right|_{N \times N}>0 \\
\forall N: \quad\left|k_{F}^{\alpha n}\left(\delta_{\alpha n \alpha^{\prime} n^{\prime}}+\pi k_{F}^{\alpha^{\prime} n^{\prime}} f_{\alpha n \alpha^{\prime} n^{\prime}}^{c}\left(k_{F}^{\alpha n}, k_{F}^{\alpha^{\prime} n^{\prime}}\right)\right)\right|_{N \times N}>0 \\
\forall N: \mid 2 k_{F}^{\alpha n}\left(\delta_{\alpha n \alpha^{\prime} n^{\prime}}+\pi k_{F}^{\alpha^{\prime} n^{\prime}} f_{\alpha n \alpha^{\prime} n^{\prime}}^{c}\left(k_{F}^{\alpha n}, k_{F}^{\alpha^{\prime} n^{\prime}}\right)\right) \\
-\left.\pi k_{F}^{\alpha n} k_{F}^{\alpha^{\prime} n^{\prime}}\left(f_{\alpha n \alpha^{\prime} n^{\prime}}^{s}\left(k_{F}^{\alpha n}, k_{F}^{\alpha^{\prime} n^{\prime}}\right)-f_{\alpha^{\prime} n^{\prime} \alpha n}^{s}\left(k_{F}^{\alpha n}, k_{F}^{\alpha^{\prime} n^{\prime}}\right)\right)\right|_{N \times N}>0
\end{array}
$$




\section{Referencias}

[1] S. Sachdev, "Quantum phase transitions of antiferromagnets and the cuprate superconductors," Lect. Notes Phys. 843 (2012) 1 doi:10.1007/978-3-642-10449-7 1 [arXiv:1002.3823 [cond-mat.str-el]].

[2] B. Keimer, S. Kivelson, M. Norman, S. Uchida and J. Zaanen, Nature 518, 179 (2015).

[3] I. Ia. Pomeranchuk, "On the Stability of a Fermi Liquid," JETP 35, 524-525 (1958).

[4] J. M. Maldacena, "The large N limit of superconformal field theories and supergravity," Adv. Theor. Math. Phys. 2 (1998) 231 [Int. J. Theor. Phys. 38 (1999) 1113] [arXiv:hep-th/9711200].

[5] S. S. Gubser, I. R. Klebanov and A. M. Polyakov, "gauge theory correlators from non-critical string theory," Phys. Lett. B 428 (1998) 105 [arXiv:hep-th/9802109].

[6] E. Witten, Adv. Theor. Math. Phys. 2 (1998) 253 [arXiv:hep-th/9802150].

[7] S. A. Hartnoll, C. P. Herzog and G. T. Horowitz, "Building a Holographic Superconductor," Phys. Rev. Lett. 101 (2008) 031601 [arXiv:0803.3295 [hep-th]].

[8] S. A. Hartnoll, C. P. Herzog and G. T. Horowitz, "Holographic Superconductors," JHEP 0812, 015 (2008) [arXiv:0810.1563 [hep-th]].

[9] S. S. Gubser and S. S. Pufu, JHEP 0811 (2008) 033 [arXiv:0805.2960 [hep-th]].

[10] S. S. Gubser, "TASI lectures: Collisions in anti-de Sitter space, conformal symmetry, and holographic superconductors," arXiv:1012.5312 [hep-th]

[11] S. S. Gubser, Phys. Rev. D 78, 065034 (2008) [arXiv:0801.2977 [hep-th]].

[12] S. S. Gubser, "Phase transitions near black hole horizons," Class. Quant. Grav. 22 (2005) 5121 [arXiv:hep-th/0505189].

[13] J. W. Chen, Y. J. Kao and W. Y. Wen, "Peak-Dip-Hump from Holographic Superconductivity," Phys. Rev. D 82, 026007 (2010) [arXiv:0911.2821 [hep-th]].

[14] S. S. Gubser, F. D. Rocha and A. Yarom, "Fermion correlators in non-abelian holographic superconductors," JHEP 1011, 085 (2010) doi:10.1007/JHEP11(2010)085 [arXiv:1002.4416 [hep-th]].

[15] B. Zwiebach, "A first course in string theory," Cambridge, UK: Univ. Pr. (2009) 673 p

[16] M. Bianchi, D. Z. Freedman and K. Skenderis, Nucl. Phys. B 631, 159 (2002) doi:10.1016/S05503213(02)00179-7 [hep-th/0112119].

[17] G.T.Horowitz and A.Strominger, 'Black string and P.Branes," Nucl.Phys.B 360 (1991) 197.

[18] Leonard Susskind and James Lindesay, 'An introduction to black holes, information and the string theory revolution"

[19] "Towards a Novel no-hair Theorem for Black Holes," Phys. Rev . D74, 084008 (2006) doi:10.1103/PhysRevD74.084008 [gr-qc/0608075]

[20] T. Albash and C. V. Johnson, "Phases of Holographic Superconductors in an External Magnetic Field," arXiv:0906.0519 [hep-th]; "Vortex and Droplet Engineering in Holographic Superconductors," arXiv:0906.1795 [hep-th].

[21] M. Montull, A. Pomarol and P. J. Silva, "The Holographic Superconductor Vortex," Phys. Rev. Lett. 103 (2009) 091601 [arXiv:0906.2396 [hep-th]].

[22] O. Domenech, M. Montull, A. Pomarol, A. Salvio and P. J. Silva, JHEP 1008, 033 (2010) [arXiv:1005.1776 [hep-th]].

[23] J. i. Koga, K. Maeda and K. Tomoda, "A holographic superconductor model in a spatially anisotropic background," Phys. Rev. D 89, 104024 (2014) [arXiv:1401.6501 [hep-th]].

[24] S. S. Gubser, "Colorful horizons with charge in anti-de Sitter space," Phys. Rev. Lett. 101 (2008) 191601 [arXiv:0803.3483 [hep-th]]. 
[25] S. Gangopadhyay and D. Roychowdhury, JHEP 1208, 104 (2012) [arXiv:1207.5605 [hep-th]].

[26] Z. Y. Nie, R. G. Cai, X. Gao and H. Zeng, "Competition between the s-wave and p-wave superconductivity phases in a holographic model," JHEP 1311, 087 (2013) [arXiv:1309.2204 [hep-th]].

[27] I. Amado, D. Arean, A. Jimenez-Alba, L. Melgar and I. Salazar Landea, "Holographic s+p Superconductors," Phys. Rev. D 89, 026009 (2014) [arXiv:1309.5086 [hep-th]].

[28] D. Momeni, M. Raza and R. Myrzakulov, "Analytical coexistence of s, p, s + p phases of a holographic superconductor," arXiv:1310.1735 [hep-th].

[29] Z. Y. Nie, R. G. Cai, X. Gao, L. Li and H. Zeng, "Phase transitions in a holographic s+p model with backreaction," arXiv:1501.00004 [hep-th].

[30] K. K. Gomes, A. N. Pasupathy, A. Pushup, S. Ono, Y. Ando, and A. Yazdani, "Visualizing pair formation pn the atomic scale in the high- $T_{c}$ superconductor $\mathrm{Bi}_{2} \mathrm{Sr}_{2} \mathrm{CaCu}_{2} \mathrm{O}_{8+\delta}$," nat, vol. 447, pp. 569-572, May 2007.

[31] A. R. Lugo and F. A, Schaposnik, Phys. Lett. B 467 (1999)43.

[32] A. R. Lugo, E. F. Moreno and F. A, Schaposnik, Phys. Lett. B 473 (2000)35.

[33] A. R. Lugo, E. F. Moreno and F. A. Schaposnik, "Holography and $A d S_{4}$ self-gravitating dyons," JHEP 1011, 081 (2010) [arXiv:1007.1482 [hep-th]].

[34] M. Ammon, J. Erdmenger, V. Grass, P. Kerner and A. O’Bannon, Phys. Lett. B 686, 192 (2010) [arXiv:0912.3515 [hep-th]].

[35] P. Basu, A. Mukherjee and H. H. Shieh, "Supercurrent: Vector Hair for an AdS Black Hole," Phys. Rev. D 79, 045010 (2009) [arXiv:0809.4494 [hep-th]].

[36] C. P. Herzog, P. K. Kovtun and D. T. Son, "Holographic model of superfluidity," Phys. Rev. D 79, 066002 (2009) [arXiv:0809.4870 [hep-th]].

[37] A. Bianchi et al., "First order superconducting phase transition in CeCoIn5," Phys. Rev. Lett. 89, 137002 (2002) [cond-mat/0203310].

[38] S. Yonezawa, T. Kajikawa and Y. Maeno, "First-order superconducting transition of Sr2 RuO4", Phys. Rev. Lett. 110, 077003 (2013) [arXiv:1212.4954].

[39] Y. Tanaka, A. Iyo, S. Itoh, K. Tokiwa, T. Nishio and T. Yanagisawa, "Experimental observation of a possible first-order phase transition below the superconducting transition temperature in the multilayer cuprate superconductor HgBa2 Ca4 Cu5 Oy ," J. Phys. Soc. Japan. 83074705 (2014) [arXiv:1408.1445].

[40] P. Basu, J. He, A. Mukherjee and H. H. Shieh, "Hard-gapped Holographic Superconductors," Phys. Lett. B 689, 45 (2010) [arXiv:0911.4999 [hep-th]].

[41] M. M. Roberts and S. A. Hartnoll, "Pseudogap and time reversal breaking in a holographic superconductor," JHEP 0808, 035 (2008) [arXiv:0805.3898 [hep-th]].

[42] Raúl E. Arias and Ignacio Salazar Landea, "Backreacting p-wave Superconductors," JHEP 0811 (2008) 033 [arXiv:1210.6823 [hep-th]].

[43] R. M. Wald, "General Relativity," Chicago, Usa: Univ. Pr. ( 1984) 491p

[44] O. Aharony, S. S. Gubser, J. M. Maldacena, H. Ooguri and Y. Oz, "Large N field theories, string theory and gravity," Phys. Rept. 323 (2000) 183 [arXiv:hep-th/9905111].

[45] J. Erdmenger, P. Kerner and S. Muller, "Towards a Holographic Realization of Homes' Law," JHEP 1210, 021 (2012) [arXiv:1206.5305 [hep-th]].

[46] M. Henningson and K. Skenderis, "The Holographic Weyl anomaly," JHEP 9807, 023 (1998) [hepth/9806087].

[47] V. Balasubramanian and P. Kraus, "A Stress tensor for Anti-de Sitter gravity," Commun. Math. Phys. 208, 413 (1999) [hep-th/9902121]. 
[48] Amin Akhavan and Mohsen Alishahiha, "P-Wave Holographic Insulator/Superconductor Phase Transition," JHEP 0811 (2008) 033 [arXiv:0805.2960 [hep-th]].

[49] G. T. Horowitz and M. M. Roberts, "Zero Temperature Limit of Holographic Superconductors," JHEP 0911, 015 (2009) [arXiv:0908.3677 [hep-th]].

[50] S. S. Gubser and F. D. Rocha, "The gravity dual to a quantum critical point with spontaneous symmetry breaking," Phys. Rev. Lett. 102, 061601 (2009) [arXiv:0807.1737 [hep-th]].

[51] R. A. Konoplya and A. Zhidenko, "Holographic conductivity of zero temperature superconductors," Phys. Lett. B 686, 199 (2010) [arXiv:0909.2138 [hep-th]].

[52] S. S. Gubser and A. Nellore, "Ground states of holographic superconductors," Phys. Rev. D 80, 105007 (2009) [arXiv:0908.1972 [hep-th]].

[53] S. Bolognesi and D. Tong, "Monopoles and Holography," JHEP 1101, 153 (2011) [arXiv:1010.4178 [hep-th]].

[54] G. L. Giordano, A. R. Lugo, "Transport coefficients from holography in a Yang-Mill-Higgs theory on $A d S_{4}$ with a gravity dual," work in progress.

[55] C. P. Herzog, K. W. Huang and R. Vaz, "Linear Resistivity from Non-Abelian Black Holes," JHEP 1411, 066 (2014) [arXiv:1405.3714 [hep-th]]

[56] Andrea Damascelli, Zahid Hussain, and Zhi-Xun Shen, "Angle-resolved photoemission studies of the cuprate superconductors" Rev. Mod. Phys. 75 (2003) 473

[57] Manfred Sigrist and Kazuo Ueda, "Phenomenological theory of unconventional superconductivity", Rev. Mod. Phys. 63 (1991) 239

[58] S. S. Gubser, I. R. Klebanov and A. M. Polyakov, "gauge theory correlators from non-critical string theory," Phys. Lett. B 428 (1998) 105 [arXiv:hep-th/9802109].

[59] E. Witten, "Anti-de Sitter space and holography," Adv. Theor. Math. Phys. 2 (1998) 253 [arXiv:hepth/9802150].

[60] S. A. Hartnoll, "Lectures on holographic methods for condensed matter physics," Class. Quant. Grav. 26, 224002 (2009) doi:10.1088/0264-9381/26/22/224002 [arXiv:0903.3246 [hep-th]].

[61] S. S. Gubser, "Phase transitions near black hole horizons," Class. Quant. Grav. 22 (2005) 5121 [arXiv:hep-th/0505189].

[62] S. S. Gubser, "Breaking an Abelian gauge symmetry near a black hole horizon," Phys. Rev. D 78, 065034 (2008) [arXiv:0801.2977 [hep-th]].

[63] M. Ammon, J. Erdmenger, V. Grass, P. Kerner and A. O'Bannon, "On Holographic p-wave Superfluids with Back-reaction," Phys. Lett. B 686, 192 (2010) [arXiv:0912.3515 [hep-th]].

[64] Raúl E. Arias and Ignacio Salazar Landea, "Backreacting p-wave Superconductors," JHEP 0811 (2008) 033 [arXiv:1210.6823 [hep-th]].

[65] C. P. Herzog, K. W. Huang and R. Vaz, "Linear Resistivity from Non-Abelian Black Holes," JHEP 1411, 066 (2014) [arXiv:1405.3714 [hep-th]]

[66] S. S. Lee, "A Non-Fermi Liquid from a Charged Black Hole: A Critical Fermi Ball," Phys. Rev. D 79, 086006 (2009) doi:10.1103/PhysRevD.79.086006 [arXiv:0809.3402 [hep-th]].

[67] H. Liu, J. McGreevy and D. Vegh, "Non-Fermi liquids from holography," Phys. Rev. D 83, 065029 (2011) doi:10.1103/PhysRevD.83.065029 [arXiv:0903.2477 [hep-th]].

[68] T. Faulkner, H. Liu, J. McGreevy and D. Vegh, "Emergent quantum criticality, Fermi surfaces, and AdS(2)," Phys. Rev. D 83, 125002 (2011) doi:10.1103/PhysRevD.83.125002 [arXiv:0907.2694 [hep-th]].

[69] M. Cubrovic, J. Zaanen and K. Schalm, "String Theory, Quantum Phase Transitions and the Emergent Fermi-Liquid," Science 325, 439 (2009) doi:10.1126/science.1174962 [arXiv:0904.1993 [hep-th]]. 
[70] T. Faulkner, N. Iqbal, H. Liu, J. McGreevy and D. Vegh, "Holographic non-Fermi liquid fixed points," Phil. Trans. Roy. Soc. A 369, 1640 (2011) doi:10.1098/rsta.2010.0354 [arXiv:1101.0597 [hep-th]].

[71] T. Faulkner, N. Iqbal, H. Liu, J. McGreevy and D. Vegh, "From Black Holes to Strange Metals," arXiv:1003.1728 [hep-th].

[72] T. Faulkner, N. Iqbal, H. Liu, J. McGreevy and D. Vegh, "Charge transport by holographic Fermi surfaces," Phys. Rev. D 88, 045016 (2013) doi:10.1103/PhysRevD.88.045016 [arXiv:1306.6396 [hep-th]].

[73] N. Iqbal, H. Liu and M. Mezei, "Lectures on holographic non-Fermi liquids and quantum phase transitions," arXiv:1110.3814 [hep-th].

[74] J. Zaanen, Y.W. Sun, Y. Liu and K. Schalm, "The AdS/CMT manual for plumbers and electricians," Universiteit Leiden, October 15, 2012; http://www.uu.nl/organisatie/faculteit-betawetenschappen

[75] P. Basu, J. He, A. Mukherjee, M. Rozali and H. H. Shieh, Can. J. Phys. 89, 231 (2011) [arXiv:1002.4929 [hep-th]].

[76] T. Faulkner, G. T. Horowitz, J. McGreevy, M. M. Roberts and D. Vegh, JHEP 1003 (2010) 121 doi:10.1007/JHEP03(2010)121 [arXiv:0911.3402 [hep-th]].

[77] S. S. Gubser, F. D. Rocha and P. Talavera, JHEP 1010 (2010) 087 doi:10.1007/JHEP10(2010)087 [arXiv:0911.3632 [hep-th]].

[78] M. Ammon, J. Erdmenger, M. Kaminski and A. O'Bannon, "Fermionic Operator Mixing in Holographic p-wave Superfluids," JHEP 1005, 053 (2010) doi:10.1007/JHEP05(2010)053 [arXiv:1003.1134 [hep-th]].

[79] D. T. Son and A. O. Starinets, " Minkowski space correlators in AdS/CFT correspondence:Recipe and applications," JHEP BF 0209, 042 (2002) doi:10.1088/1126-6708/2002/09/042. [hep-th/0205051]

[80] N. Iqbal and H. Liu, "Real-time response in AdS/CFT with application to spinors," Fortsch. Phys. 57, 367 (2009) doi:10.1002/prop.200900057 [arXiv:0903.2596 [hep-th]].

[81] G. L. Giordano and A. R. Lugo, "Holographic phase transitions from higgsed, non abelian charged black holes," JHEP 1507, 172 (2015) doi:10.1007/JHEP07(2015)172 [arXiv:1501.04033 [hep-th]].

[82] A. R. Lugo, E. F. Moreno and F. A. Schaposnik, "Holography and $A d S_{4}$ self-gravitating dyons," JHEP 1011, 081 (2010) [arXiv:1007.1482 [hep-th]].

[83] A. R. Lugo, E. F. Moreno and F. A. Schaposnik, "Holographic Phase Transition from Dyons in an AdS Black Hole Background," JHEP 1003, 013 (2010) [arXiv:1001.3378 [hep-th]].

[84] M. Henningson and K. Sfetsos, "Spinors and the AdS / CFT correspondence," Phys. Lett. B 431, 63 (1998).

[85] M. Henneaux, "Boundary terms in the AdS / CFT correspondence for spinor fields," In *Tbilisi 1998, Mathematical methods in modern theoretical physics* 161-170 [hep-th/9902137].

[86] L. D. Landau, E. M. Lifshitz, L. P. Pitaevski $\breve{i}$, "Statistical Physics, Part 2," Pergamon Press, Oxford, U.K. (1981)

[87] X. Arsiwalla, J. de Boer, K. Papadodimas and E. Verlinde, "Degenerate Stars and Gravitational Collapse in AdS/CFT," JHEP 1101, 144 (2011) [arXiv:1010.5784 [hep-th]].

[88] J. G. Bednorz and K. A. Muller, Zeitschrift fur Physik B Condensed Matter 64, 189 (1986)

[89] J. Bardeen, L. N. Cooper, and J. R. Schrieffer, Phys. Rev. 106, 162 (1957).

[90] B. Keimer, S. Kivelson, M. Norman, S. Uchida, and J. Zanner, Nature 518, 179 (2015)

[91] E. Fradkin, S. A. Kivelson, and J. M. Tranquada, Rev. Mod. Phys. 87, 457 (2015)

[92] U. Chatterjee, D. Ai, J. Zhao, S. Rosenkranz, A. Kaminski, H. Raffy, Z. Li, K. Kadowaki, M. Randeria, M. R. Norman, et al ., Proceedings of the National Academy of Sciences 108, 9346 (2011).

[93] J. Maldacena, Adv. Theor. Math, Phys. 2, 231 (1998).

[94] E. Witten, Adv. Theor. Math. Phys. 2, 253 (1998). 
[95] S. A. Hartnoll, Classical Quan. Grav. 26, 224002 (2009).

[96] J. McGreevy, Ad. High Energy Physc. 2010

[97] J. Zaanen, Y. Liu, Y.-W. Sun, and K. Schalm, Holographic Duality in COndensed Matter Physics (Cambridge University Press, 2015).

[98] M. Rangamani, Classical Quan. Grav. 26, 224003 (2009).

[99] P. Kovtum, J. Phys. A: Math. Theor. 45, 473001 (2012).

[100] P. Kovtum, D. T. Son, and A. O. Starinets, J. High Energy Phys. 2003, 064 (2003).

[101] S. S. Gubser, Phys. Rev. D 78, 065034 (2008)

[102] S. A. Hartnoll, C. P. Herzog, and G. T. Horowitz, J. High Energy Phys. 2008, 015 (2008).

[103] C. P. Herzog, J. Phys. A: Math. Theor. 42, 343001 (2009)

[104] S. Ryu and T. Takayanagi, Phys. Rev. Lett. 96, 181602 (2006).

[105] S. Ryu and T. Takayanagi, J. High Energy Phys. 2006, 045 (2006).

[106] H. Casini, M. Huerta, and R. C. Myers, J. High Energy Phys. 2011, 1 (2011).

[107] A. Lewkowyc and J. Maldacena, J. High Energy Phys. 2013, 1 (2013).

[108] E. Kiritsis and L. Li, "Holographic Competition of Phases and Superconductivity," JHEP 1601, 147 (2016) doi:10.1007/JHEP01(2016)147 [arXiv:1510.00020 [cond-mat.str-el]].

[109] T. Faulkner, H. Liu, J. McGreevy and D. Vegh, "Emergent quantum criticality, Fermi surfaces, and AdS(2)," Phys. Rev. D 83 (2011) 125002 doi:10.1103/PhysRevD.83.125002 [arXiv:0907.2694 [hep-th]].

[110] T. Faulkner, N. Iqbal, H. Liu, J. McGreevy and D. Vegh, "Holographic non-Fermi liquid fixed points," Phil. Trans. Roy. Soc. A 369, 1640 (2011) doi:10.1098/rsta.2010.0354 [arXiv:1101.0597 [hep-th]].

[111] G. L. Giordano, N. E. Grandi and A. R. Lugo, "Fermionic spectral functions in backreacting p-wave superconductors at finite temperature," arXiv:1610.04268 [hep-th].

[112] See for example section 1.3 in, O. Aharony, S. S. Gubser, J. M. Maldacena, H. Ooguri and Y. Oz, "Large N field theories, string theory and gravity," Phys. Rept. 323, 183 (2000) [hep-th/9905111], and references.

[113] R. G. Cai, Y. H. Qi, Y. L. Wu and Y. L. Zhang, "Topological Non-Fermi Liquid," arXiv:1601.03865 [hep-th].

[114] Subir Sachev "Quantum Phase Transitions " Cambridge University Press

[115] S. A. Hartnoll and A. Tavanfar, Phys. Rev. D 83, 046003 (2011) doi:10.1103/PhysRevD.83.046003 [arXiv:1008.2828 [hep-th]].

[116] Damien Bertrand "A Relativistic BCS Theory of Superconductivity: An experimentally Motivated Study of Electric Fields in Superconductors"

[117] S. A. Hartnoll, D. M. Hofman and D. Vegh, JHEP 1108, 096 (2011) doi:10.1007/JHEP08(2011)096 [arXiv:1105.3197 [hep-th]].

[118] A. Allais, J. McGreevy and S. J. Suh, Phys. Rev. Lett. 108, 231602 (2012) doi:10.1103/PhysRevLett.108.231602 [arXiv:1202.5308 [hep-th]].

[119] V. G. M. Puletti, S. Nowling, L. Thorlacius and T. Zingg, JHEP 1101, 117 (2011) doi:10.1007/JHEP01(2011)117 [arXiv:1011.6261 [hep-th]].

[120] S. A. Hartnoll and P. Petrov, Phys. Rev. Lett. 106, 121601 (2011) doi:10.1103/PhysRevLett.106.121601 [arXiv:1011.6469 [hep-th]].

[121] Damien Bertrand "Electron star birth: A continuous phase transition at nonzero density," 


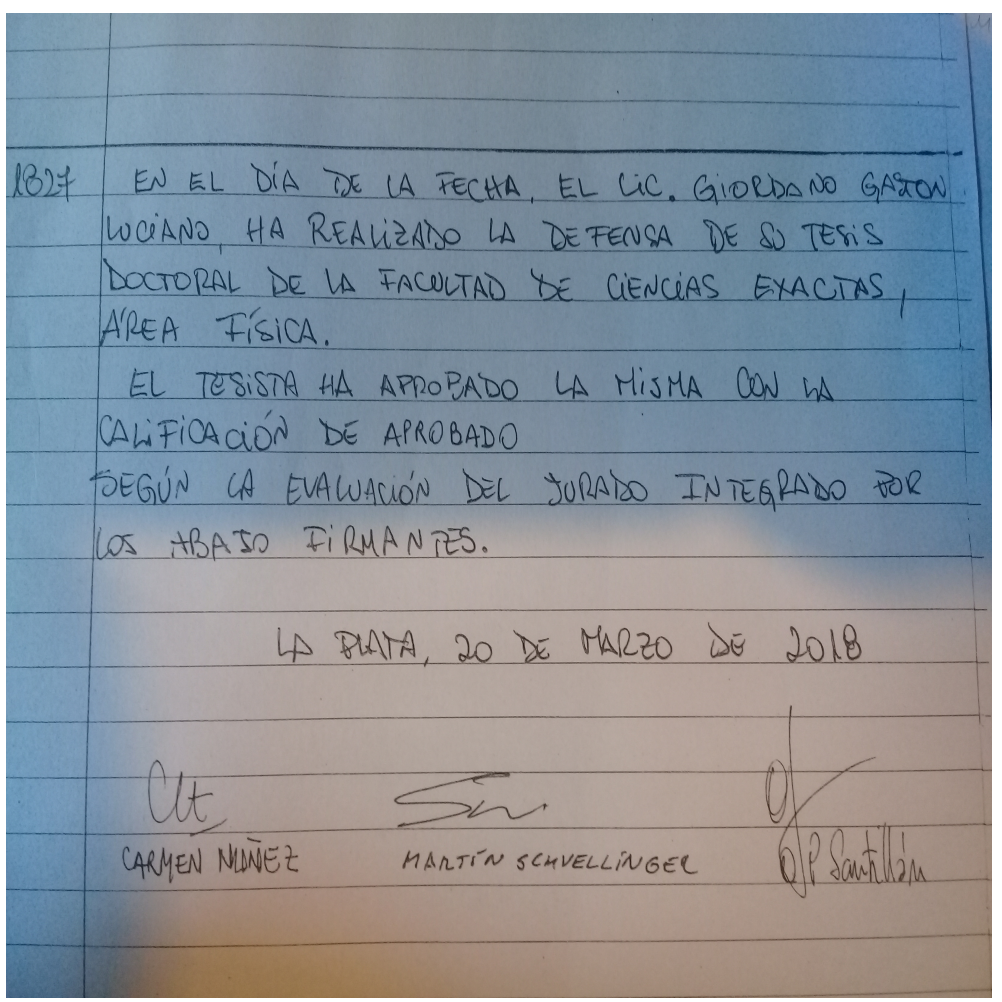

Figura 58: 